\title{
Fault Recovery of an Under-Actuated Quadrotor Aerial Vehicle
}

\author{
Mina Ranjbaranhesarmaskan
}

\author{
A Thesis \\ in \\ The Department \\ of \\ Electrical and Computer Engineering \\ Presented in Partial Fulfillment of the Requirements \\ for the Degree of Master of Applied Science at \\ Concordia University \\ Montréal, Québec, Canada
}

April 2010

(C) Mina Ranjbaranhesarmaskan, 2010 
Library and Archives
Canada

Published Heritage

Branch

395 Wellington Street Ottawa ON K1A ON4

Canada
Bibliothèque et

Archives Canada

Direction du

Patrimoine de l'édition

395 , rue Wellington

Ottawa ON K1A ON4

Canada
Your îie votre réiérence

ISBN: 978-0-494-67092-7

Our file Notre reférence

ISBN: 978-0-494-67092-7

\section{NOTICE:}

The author has granted a nonexclusive license allowing Library and Archives Canada to reproduce, publish, archive, preserve, conserve, communicate to the public by telecommunication or on the Internet, loan, distribute and sell theses worldwide, for commercial or noncommercial purposes, in microform, paper, electronic and/or any other formats.

The author retains copyright ownership and moral rights in this thesis. Neither the thesis nor substantial extracts from it may be printed or otherwise reproduced without the author's permission.
AVIS:

L'auteur a accordé une licence non exclusive permettant à la Bibliothèque et Archives Canada de reproduire, publier, archiver, sauvegarder, conserver, transmettre au public par télécommunication ou par l'Internet, prêter, distribuer et vendre des thèses partout dans le monde, à des fins commerciales ou autres, sur support microforme, papier, électronique et/ou autres formats.

L'auteur conserve la propriété du droit d'auteur et des droits moraux qui protège cette thèse. $\mathrm{Ni}$ la thèse ni des extraits substantiels de celle-ci ne doivent être imprimés ou autrement reproduits sans son autorisation.
In compliance with the Canadian Privacy Act some supporting forms may have been removed from this thesis.

While these forms may be included in the document page count, their removal does not represent any loss of content from the thesis.
Conformément à la loi canadienne sur la protection de la vie privée, quelques formulaires secondaires ont été enlevés de cette thèse.

Bien que ces formulaires aient inclus dans la pagination, il n'y aura aucun contenu manquant.

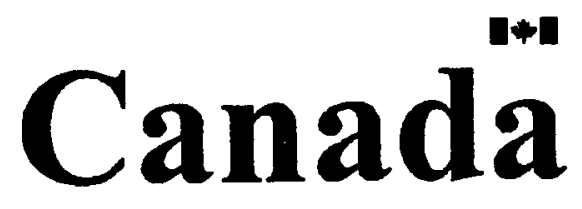




\title{
CONCORDIA UNIVERSITY
}

\author{
School of Graduate Studies
}

This is to certify that the thesis proposal prepared

By: $\quad$ Mina Ranjbaranhesarmaskan

Entitled: Fault Recovery of an Under-Actuated Quadrotor Aerial Vehicle

and submitted in partial fulfilment of the requirements for the degree of

\section{Master of Applied Science}

complies with the regulations of this University and meets the accepted standards with respect to originality and quality.

Signed by the final examining committee:

Dr. A.G Aghdam, Chair

Dr. M. Chen, External examiner

Dr. S. Hashtrudi Zad, Examiner

Dr. K. Khorasani, Supervisor

Approved by

Chair of the Department of Electrical and Computer Engineering

Dean of the Faculty of Engineering and Computer Science 


\section{ABSTRACT \\ Fault Recovery of an Under-Actuated Quadrotor Aerial Vehicle Mina Ranjbaranhesarmaskan}

The research on autonomous flying robots has intensified considerably due to recent growth of civilian and military interests in Unmanned Aerial Vehicles (UAV). Miniature UAVs with the ability to vertically take off and land such as quadrotor aerial vehicles exhibit further advantages and features in maneuverability that have recently gained interest among the research community.

Reliability of control systems require robustness and fault tolerance in presence of anomalies and unexpected failures in actuators, sensors or subsystems. Autonomy of dynamical systems that are vulnerable to the above failures has been an important topic of research during the past several years. Particularly, in small aerial vehicles due to hardware redundancy limitations design of a reliable control system plays an important role in ensuring acceptable and efficient performance.

In view of the above, an autonomous recovery from actuators faults in underactuated quadrotor aerial vehicles constitutes the main focus of the research investigated in this dissertation. A self-recovery mechanism, which extends the capabilities of the quadrotor system to operate under the presence of actuator faults is developed. The solution proposed takes into account the management of the control authority in the system by taking advantage of the post-fault model of an actuator.

The first step in accomplishing this task is achieved by developing a controller under healthy condition that guarantees the stability of the quadrotor system in response to the commanded trajectories. This controller is then extended to incorporate the effects of a certain type of actuators fault by estimating the post-fault model of the system and then by properly commanding the faulty actuators accordingly. The performance of the proposed fault recovery scheme in presence of noise in the input and output channels and under different fault severities is evaluated 
through numerical simulations. It is shown that a significant reduction in the average tracking steady state errors are obtained through the application of the proposed recovery mechanism. The proposed scheme is applicable to rotorcraft systems even in presence of multiple faults in actuators. 


\section{To my parents}

for their love and support 


\section{ACKNOWLEDGEMENTS}

This work would not be possible without help and encouragement I received

from several people. First and foremost, I would like to express my most sincere gratitude to my supervisor, Dr. Khashayar Khorasani for his continuous patience, support and guidance in the development of this thesis.

My gratitude extends to my colleagues at Concordia University and my friends in Montreal whose friendship means a lot to me. I would especially like to express my gratitude toward my dear friend Kian, for being there for me always supportive and encouraging.

And finally, I thank my dear family, whom have always been present through out my life in spite of the distance. 


\section{TABLE OF CONTENTS}

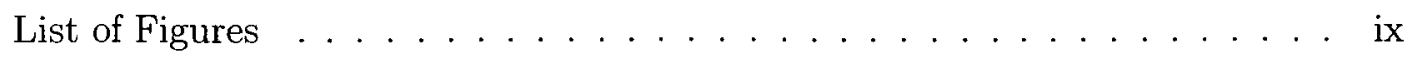

List of Tables ......................... . . . . . . . . . . .

List of Symbols . . . . . . . . . . . . . . . xv

1 Introduction 1

1.1 Problem Statement . . . . . . . . . . . . . . . 2

1.2 Literature Review . . . . . . . . . . . . . . . . . . 3

1.2.1 Fault Detection, Isolation and Recovery . . . . . . . . 3

1.2.2 Fault Recovery . . . . . . . . . . . . . . . . 4

1.2.3 Controlling the Quadrotor . . . . . . . . . . . 8

1.3 Thesis Contribution . . . . . . . . . . . . . . 11

1.4 Thesis Outline . . . . . . . . . . . . . . . . . . . 12

2 Mathematical Model of the Quadrotor System 14

2.1 Basic Concepts ... . . . . . . . . . . . . . . 14

2.2 Newton-Euler Model . . . . . . . . . . . . . . . . . . . 17

2.2.1 Kinematics Model . . . . . . . . . . . . . . 18

2.2 .2 Dynamic Models . . . . . . . . . . . . . . 21

2.3 The DC Motor Actuator . . . . . . . . . . . . . . . . 30

2.4 Conclusions . . . . . . . . . . . . . . . . . 33

3 Control Design for the Healthy Quadrotor System 34

3.1 Control Modeling . . . . . . . . . . . . . . . 35

3.2 Moment Dynamics . . . . . . . . . . . . . . . . 37

3.3 Development of Control Schemes for the Healthy Quadrotor . . . . 42

3.3 .1 LQR Controller . . . . . . . . . . . . . . . . . . 43

3.3.2 Feedback Linearization Control Design . . . . . . . . . . . 46 
3.3.3 Quadrotor Control Scheme Design . . . . . . . . . . . . 52

3.4 Simulation Results . . . . . . . . . . . . . . . . . . 59

3.4.1 Healthy System Response to 1st Trajectory . . . . . . . . . 60

3.4.2 Healthy System Response to 2nd Trajectory . . . . . . . . 62

3.5 Conclusions . . . . . . . . . . . . . . 63

4 Fault Recovery of the Quadrotor System $\quad 77$

4.1 Faults in the Quadrotor Actuators . . . . . . . . . . . . . . . 77

4.2 Partial LOE Fault Modeling . . . . . . . . . . . . . . . 80

4.3 Implementation of a Fault Recovery System . . . . . . . . . . . 83

4.4 Fault Recovery Module . . . . . . . . . . . . . . . . 85

4.4.1 Adaptive Feedback Linearization . . . . . . . . . . . . . 85

4.4.2 Generalization of the Adaptive Feedback Linearization . . . . 94

4.5 LIP, Float and HOF Faults Modeling . . . . . . . . . . . . . . . 99

4.6 Simulation Results . . . . . . . . . . . . . . . . . 104

4.7 Conclusions . . . . . . . . . . . . . . . . 110

5 Conclusions and Future Work $\quad 139$

5.1 Conclusions . . . . . . . . . . . . . . . . . . . 139

5.2 Future work ........................... 141 


\section{List of Figures}

2.1 Simplified quadrotor model at hovering and coordinate systems (body and earth frame) .......................... 15

2.2 Quadrotor movement concepts; (a) Going up; (b) Moving right; (c) Moving forward; (d) Rotating left; (e) and Rotating right. . . . . . . 16

2.3 Quadrotor rotation; (a) Roll; (b) Pitch; and (c) Yaw. . . . . . . . . . 19

2.4 DC motor model . . . . . . . . . . . . . . . . 30

3.1 The schematic of the set-point optimal LQR control. . . . . . . . . 45

3.2 Linear position response to the commanded 1st trajectory : (a) $x$, (b) $y$ and $($ c) $z$ measured in meters. . . . . . . . . . . . . 65

3.3 Euler angles in response to the commanded 1st trajectory : (a) Roll (rad), (b) Pitch (rad) and (c) Yaw (rad). . . . . . . . . . . 66

3.4 Input voltage to the 4 propellers in response to the commanded 1st trajectory. ....................... 67

3.5 Input moments $U_{1}, U_{2}, U_{3}$ and $U_{4}$ to the dynamics of the quadrotor system in response to the commanded 1st trajectory. . . . . . 68

3.6 Error signals $e_{x}, e_{y}, e_{z}$ measured in meters and $e_{\psi}$ in radian in response to the commanded 1st trajectory. . . . . . . . . . . . 69

3.7 Error signals $e_{x}, e_{y}, e_{z}$ and $e_{\psi}$ measured in meters and $e_{\psi}$ in radian in response to the commanded 1st trajectory with $10 \%$ variations in the body inertia parameters. . . . . . . . . . . . . 70

3.8 Linear position response to the commanded 2nd trajectory : (a) $x$, (b) $y$ and (c) $z$ measured in meters. . . . . . . . . . 71

$3.93-\mathrm{D}$ representation of the linear position response to the commanded 2nd trajectory. ........................... 72 
3.10 Euler angles in response to the commanded 2nd trajectory : (a) Roll (rad), (b) Pitch (rad) and (c) Yaw (rad). . . . . . . . . . 73

3.11 Input voltage to the 4 propellers in response to the commanded 2nd trajectory. ........................ 74

3.12 Input moments $U_{1}, U_{2}, U_{3}$ and $U_{4}$ to the dynamics of the quadrotor system in response to the commanded 2 nd trajectory. . . . . . 75

3.13 Error signals $e_{x}, e_{y}, e_{z}$ measured in meters and $e_{\psi}$ in radian in response to the commanded 2 nd trajectory. . . . . . . . . 76

4.1 Quadrotor augmented control system with a fault recovery functionality. 84

4.2 Linear position in response to the commanded trajectory corresponding to a $10 \%$ LOE fault in the first actuator and without fault recovery mechanism: (a) $\mathrm{x}$, (b) $\mathrm{y}$ and (c) $\mathrm{z}$, measured in meters. . . . . . 111

4.3 Euler angles in response to the commanded trajectory corresponding to a $10 \%$ LOE fault in the first actuator and without fault recovery mechanism: (a) Roll (rad), (b) Pitch (rad) and (c) Yaw (rad). . . . . 112

4.4 Input signals in response to the commanded trajectory corresponding to a $10 \%$ LOE fault in the first actuator and without fault recovery

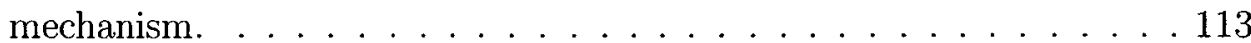

4.5 Error signals $e_{x}, e_{y}, e_{z}$ measured in meters and $e_{\psi}$ in radians in response to the commanded trajectory corresponding to a $10 \% \mathrm{LOE}$ fault in the first actuator and without fault recovery mechanism. . . 114

4.6 Linear position in response to the commanded trajectory corresponding to a $10 \%$ LOE fault in the first actuator and with the fault recovery mechanism: (a) x, (b) y and (c) z, measured in meters. . . . . 115

4.7 Euler angles in response to the commanded trajectory corresponding to a $10 \%$ LOE fault in the first actuator and with the fault recovery mechanism: (a) Roll (rad), (b) Pitch (rad) and (c) Yaw (rad). . . . . 116 
4.8 Input signals in response to the commanded trajectory corresponding to a $10 \%$ LOE fault in the first actuator with the fault recovery mechanism. . . . . . . . . . . . . . . . 117

4.9 Estimated parameters in response to the commanded trajectory corresponding to a $10 \% \mathrm{LOE}$ fault in the first actuator with the fault recovery mechanism: (a) $A_{t 1}$ and (b) $B_{t 1} \ldots \ldots 118$

4.10 Error signals $e_{x}, e_{y}, e_{z}$ measured in meters and $e_{\psi}$ in radians in response to the commanded trajectory corresponding to a $10 \% \mathrm{LOE}$ fault in the first actuator with the fault recovery mechanism. . . . . 119

4.11 Linear position in response to the commanded trajectory corresponding to a $25 \% \mathrm{LOE}$ fault in the first actuator with the fault recovery mechanism: (a) $\mathrm{x}$, (b) y and (c) z, measured in meters. . . . . . . 120

4.12 Euler angles in response to the commanded trajectory corresponding to a $25 \%$ LOE fault in the first actuator with the fault recovery mechanism: (a) Roll (rad), (b) Pitch (rad) and (c) Yaw (rad). . . . . 121

4.13 Input signals in response to the commanded trajectory corresponding to a $25 \%$ LOE fault in the first actuator with the fault recovery mechanism. . . . . . . . . . . . . . . . 122

4.14 Estimated parameters in response to the commanded trajectory corresponding to a $\% 25 \mathrm{LOE}$ fault in the first actuator with the fault recovery mechanism: (a) $A_{t 1}$ and (b) $B_{t 1} \ldots \ldots . \ldots 123$

4.15 Error signals $e_{x}, e_{y}, e_{z}$ measured in meters and $e_{\psi}$ in radians in response to the commanded trajectory corresponding to a $25 \% \mathrm{LOE}$ fault in the first actuator with the fault recovery mechanism. . . . . 124

4.16 Linear position in response to the commanded trajectory corresponding to a $50 \%$ LOE fault in the first actuator with the fault recovery mechanism: (a) $x$, (b) $y$ and (c) z, measured in meters. . . . . . . 125 
4.17 Euler angles in response to the commanded trajectory corresponding to a $50 \%$ LOE fault in the first actuator with the fault recovery mechanism: (a) Roll (rad), (b) Pitch (rad) and (c) Yaw (rad). . . . 126

4.18 Input signals in response to the commanded trajectory corresponding to a $50 \%$ LOE fault in the first actuator with the fault recovery

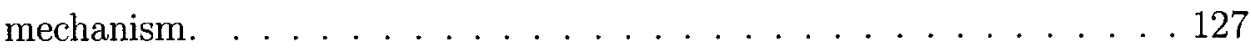

4.19 Estimated parameters in response to the commanded trajectory corresponding to a $50 \% \mathrm{LOE}$ fault in the first actuator with the fault recovery mechanism: (a) $A_{\iota 1}$ and (b) $B_{t 1} \ldots \ldots 128$

4.20 Error signals $e_{x}, e_{y}, e_{z}$ measured in meters and $e_{\psi}$ in radians in response to the commanded trajectory corresponding to a $50 \% \mathrm{LOE}$ fault in the first actuator with the fault recovery mechanism. . . . 129

4.21 Linear position in response to the commanded trajectory corresponding to a $10 \%$ LOE fault in the first and 4 th actuator and without the fault recovery mechanism: (a) $\mathrm{x}$, (b) y and (c) z, measured in meters. 130

4.22 Euler angles in response to the commanded trajectory corresponding to a $10 \%$ LOE fault in the first and 4th actuators and without the fault recovery mechanism: (a) Roll (rad), (b) Pitch (rad) and (c) Yaw

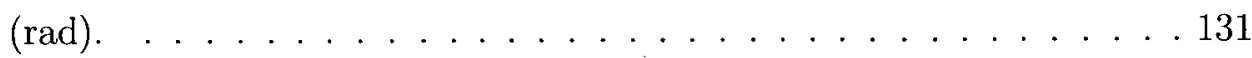

4.23 Input signals in response to the commanded trajectory corresponding to a $10 \%$ LOE fault in the first and 4th actuators without the fault recovery mechanism. . . . . . . . . . . . . . . 132

4.24 Error signals $e_{x}, e_{y}, e_{z}$ measured in meters and $e_{\psi}$ in radians in response to the commanded trajectory corresponding to a $10 \% \mathrm{LOE}$ fault in the first and 4th actuators without the fault recovery mech-

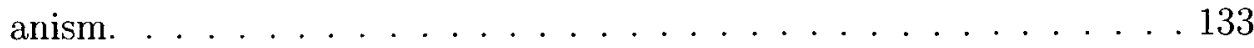


4.25 Linear position in response to the commanded trajectory corresponding to a $10 \%$ LOE fault in the first and 4th actuator and with the fault recovery mechanism: (a) $\mathrm{x}$, (b) $\mathrm{y}$ and (c) $\mathrm{z}$, measured in meters. 134

4.26 Euler angles in response to the commanded trajectory corresponding to a $10 \%$ LOE fault in the first and 4 th actuators and with the fault recovery mechanism: (a) Roll (rad), (b) Pitch (rad) and (c) Yaw (rad). 135

4.27 Input signals in response to the commanded trajectory corresponding to a $10 \%$ LOE fault in the first and 4 th actuators with the fault recovery mechanism. . . . . . . . . . . . . . 136

4.28 Estimated parameters in response to the commanded trajectory corresponding to a $10 \%$ LOE fault in the first and 4 th actuators with the fault recovery mechanism: (a) $A_{t 1}$ and (b) $B_{t 1} \ldots \ldots 137$

4.29 Error signals $e_{x}, e_{y}, e_{z}$ measured in meters and $e_{\psi}$ in radians in response to the commanded trajectory corresponding to a $10 \% \mathrm{LOE}$ fault in the first and 4th actuators with the fault recovery mechanism. 138 


\section{List of Tables}

3.1 OS4 Quadrotor physical parameters [1] . . . . . . . . . . . . 59

3.2 OS4 Quadrotor physical parameters [1] . . . . . . . . . . 60

3.3 Mean and standard deviation (Stdv) of the error signals for the $1^{\text {st }}$

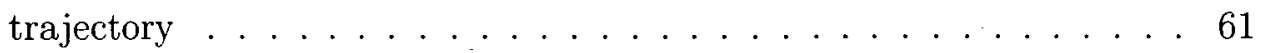

3.4 Mean and standard deviation (Stdv) of the error signals for the $1^{\text {st }}$ trajectory with $10 \%$ variations in the body inertia parameters . . . . 62

3.5 Mean and standard deviation (Stdv) of the error signals for the $2^{\text {nd }}$ trajectory. ....................... 63

3.6 Second Trajectory . . . . . . . . . . . . . . . . 64

4.1 Mean and standard deviation (Stdv) of the error signals . . . . . . 108

4.2 Mean of the input voltage to the actuators . . . . . . . . . . 109 


\section{List of Symbols}

\begin{tabular}{|c|c|}
\hline$\Omega_{i}\left(\operatorname{rads}^{-1}\right)$ & Propeller rotation speed \\
\hline$\Omega_{H}\left(\operatorname{rads}^{-1}\right)$ & Propeller rotation speed at hover \\
\hline$\Omega_{T}\left(\operatorname{rads}^{-1}\right)$ & Overall residual propeller angular speed \\
\hline$x, y, z(m)$ & Linear position \\
\hline$\phi, \theta, \psi(r a d)$ & Roll, pitch and yaw angles \\
\hline$u, v, w$ & Linear velocities \\
\hline$p, q, r$ & Angular velocities \\
\hline$m(K g)$ & Quadrotor mass \\
\hline$I_{x x}\left(K g m^{2}\right)$ & Inertia on $x$ axis \\
\hline$I_{y y}\left(K g m^{2}\right)$ & Inertia on $y$ axis \\
\hline$I_{z z}\left(K g m^{2}\right)$ & Inertia on $z$ axis \\
\hline$g\left(m s^{-2}\right)$ & Acceleration due to gravity \\
\hline$J_{p}\left(N m s^{-2}\right)$ & Propeller inertia \\
\hline$J_{m}\left(N m s^{-2}\right)$ & Motor inertia \\
\hline$J_{t p}\left(N m s^{-2}\right)$ & Total rotor inertia seen by the motor \\
\hline$b$ & Thrust coefficient \\
\hline$d$ & Drag coefficient \\
\hline$U$ & Input moment \\
\hline$u$ & Motor input voltage \\
\hline$R_{m o t}(O h m s)$ & Motor internal resistance \\
\hline$L(H)$ & Motor internal inductance \\
\hline$M_{t}(N m)$ & Motor torque \\
\hline$M_{l}(N m)$ & Load torque \\
\hline$\omega_{m}\left(\operatorname{rads}^{-1}\right)$ & Motor angular speed \\
\hline$k_{t}\left(N m A^{-1}\right)$ & Motor torque constant \\
\hline
\end{tabular}




$\begin{array}{ll}k_{e}\left(V s \mathrm{rad}^{-1}\right) & \text { Motor back-EMF constant } \\ r & \text { Gearbox reduction ratio } \\ l(m) & \text { Arm length } \\ \eta & \text { Gearbox efficiency }\end{array}$




\section{Chapter 1}

\section{Introduction}

Unmanned Aerial Vehicles, or UAVs are becoming widely used as valuable tools in today's society. These vehicles provide an added measure of safety and convenience when applied to numerous situations that previously required a full-sized aircraft with pilot.

Unmanned vehicles are important when it comes to performing a desired task in a dangerous or unaccessible environment. Unmanned indoor and outdoor mobile robots have been successfully used for some decades [2].

As their application both in the military and in the industrial sector increases, potential miniature UAVs have constantly gained interest among the research community. Mostly used for surveillance and inspection tasks, building exploration or missions in unaccessible or dangerous environments, the easy handling of the UAVS by an operator without hours of training is of paramount interest [3].

UAVs have several basic advantages over manned systems including increased manoeuvrability, low cost, reduced radar signatures and less risk to crews. Vertical take off and landing type UAVs exhibit further advantages in manoeuvrability features. Such vehicles are to require little human intervention from the take-off to the landing [4]. 
Quadrotors have become an exciting new area of unmanned aerial vehicle research in the last few years. It is an aircraft that is lifted and propelled by four rotors in a cross configuration and its basic motions are generated by varying the speeds of all the four rotors. The quadrotor rotorcraft is not a new configuration. It already existed in the year 1922 [5]. The uniqueness of this type of UAV is in its vertical landing/take off capability, hovering ability, great maneuverability and being simple to manufacture.

The quadrotor is a 6 Degree of Freedom (DOF) device with only four actuators, which make it an under-actuated vehicle with unstable dynamics and highly coupled states. In order to develop a reliable control to guarantee the capability of a stable autonomous flight, the development of simple and robust control laws stabilizing the quadrotor becomes more and more important.

Furthermore, more reliability and safety of the system due to occurrence of faults in actuators are shown to be achieved by incorporating Fault Diagnosis, Isolation and Recovery (FDIR) mechanisms in the design of the control system.

The present work is focused on the controller design and a model-based actuator fault recovery for the under-actuated quadrotor vehicle. As in an under actuated systems the degrees of freedom are more than the actuators and no redundant actuator exists to cover the performance of the faulty one. Therefore, only certain types of faults could be recovered.

\subsection{Problem Statement}

The purpose of this work is to design a controller to stabilize the under actuated quadrotor model and then to propose a recovery mechanism for the quadrotor that is subject to a fault in one or more of the actuators to ensure stability of the system. 


\subsection{Literature Review}

\subsubsection{Fault Detection, Isolation and Recovery}

A Fault Detection, Isolation and Recovery (FDIR) module is in charge of detecting, identifying, isolating and generating a mechanism to allow acceptable performance of the system that is subject to a fault. The main objective of this module is to enhance the reliability, performance and survivability of the system. Some main concepts used in the area of FDIR are listed below.

- Fault refers to an unexpected change in a system, such as component malfunction and variations in operating conditions, that tend to degrade overall system performance.

- Failure refers to the situation where a component has stopped working.

- Fault detection is the determination of the presence of faults in a system.

- Fault isolation is the determination of the origin, location and the time of the detected fault.

- Fault identification corresponds to the recognition of the type, size, nature and behavior of the detected fault.

- Fault diagnosis includes the fault isolation and identification modules and tasks.

- Fault recovery refers to the reconfiguration of the system using healthy or the available components and actuators/sensors.

A general classification of the types of the faults can be summarized as follows $[6]$ : 
- Gross parameter changes in a model, which arise when there is a disturbance entering the process from the environment through one or more independent variables.

- Structural changes, which refers to changes in the process itself and occur due to hard failures in the equipment.

- Malfunctioning sensors and actuators, which lead to deviation of the plant state variables beyond acceptable ranges.

In general, the methods for implementing fault detection are classified into two categories, namely process-history based method and process-model based method [6]. The first approach depends on the knowledge processed from past experiences and the availability of large amount of historical data, while the latter relies on interactions between various dynamical system components and variables and a priori knowledge about the process.

\subsubsection{Fault Recovery}

The goal of the fault recovery mechanism is to select an optimal possible configuration of non-faulty components in a system where a fault has occurred and diagnosed, to maintain the quality of the performance of the system despite the presence of faults.

Over the past decade many researchers have proposed different approaches for the fault recovery problem. Broadly speaking, these methods fall into two main categories [7], [8]:

- Passive methods which make use of robust control techniques to ensure that a closed-loop system remains insensitive for certain types of structural failures that can be modeled as uncertainty regions around a nominal model. Any 
failure which does not push the system outside of the stability radius given by the robust controller will still have satisfactory stability and performance. However, the drawback of this method is that, any controller with a large enough stability radius to encompass most failure situations will likely be unnecessarily conservative and there is no guarantee that unanticipated or multiple failures could be handled or even that such a controller exists. On the other hand, there are also many types of common failures, such as actuator or sensor faults, which cannot be adequately modeled as uncertainty.

- The active or reconfigurable methods differentiate themselves from passive approaches in that they take fault information explicitly into account and do not assume a static nominal model.

The existing active methods for the fault recovery problem are classified into the following approaches [9], [7]:

\section{- Multiple Model}

There are basically three methods that fall under the heading of multiple model control:

- Multiple Model Switching and Tuning (MMST) [10], [11]:

- Interacting Multiple Model (IMM) [12], and

- Propulsion Controlled Aircraft (PCA) [13].

In the first two cases all expected failure scenarios are enumerated during a Failure Modes and Effects Analysis (FMEA) and fault models constructed which cover each situation. When a failure occurs MMST switches to a precomputed control law corresponding to the current failure situation. Rather than using the model which is closest to the current failure scenario, IMM computes a fault model as a convex combination of all pre-computed fault 
models and then uses this new model to make control decisions. PCA is a special case of MMST, where the only anticipated fault is a total hydraulics failure, and in this case only the engines are used for control.

\section{- Adaptive Control Methods}

The following methods fall under this category of active approach to fault recovery problem:

- Model Reference Adaptive Control (MRAC) or Model Following method

- Adaptive feedback linearization

An adaptive controller is a controller with adjustable parameters and a mechanism for adjusting those parameters. Model following is an attractive candidate for the redesign process associated with fault tolerant control, because the goal is to emulate the performance characteristics of a desirable model [8]. In this approach a desired reference model is assumed, then the control laws of the system vary adaptively so that the output of the system in the healthy or faulty mode tracks that of the desired one. In the indirect adaptation approach, by using a parameter estimation technique, the new parameters of the faulty system are estimated and then the control law is designed so that the output matches the desired one. Direct adaptive control attempts to estimate the controller parameters directly rather than first computing the model parameters. This method is fully addressed in [14] and [15].

One of the nonlinear control tools available for the fault recovery problem is feedback linearization [7], [8], [16]. The main idea behind feedback linearization is to transform the nonlinear system into a linear one through a change of coordinates and nonlinear feedback. If feedback linearization is feasible [17], it is possible to achieve first cancellation of the nonlinear function and second, desired closed loop performance through the application of linear control 
methodologies. In this approach the faults are identified indirectly by estimating the parameters of the system, and then these estimated parameters are used to update the new parameters of the controller. Artificial neural networks or least-square parameter estimation are some of the techniques used to estimate the post-fault model of the system.

\section{- Control Allocation Problem}

Control allocation is the problem of producing a desired set of forces and moments from a (usually large) set of actuators [7]. In fault recovery problem, the idea of this method is to use the redundancy of the operable actuators to cancel the effect of the failed ones due to the fault in the system. Clearly, the greater the control redundancy, the better this approach is suited for [18]. References [19] and [20] contain a list of recent work on control allocation and provides exploratory discussions on several control allocation approaches based on quadratic and linear programming methods. Application of control allocation in fault recovery is also addressed in [18], [21], [22] and [23].

\section{- Model Predictive Control (MPC)}

Model predictive control has been proposed as a method for reconfiguration due to its ability to handle constraints and changing model dynamics systematically [7]. Since MPC relies on an internal model of the system a fault model is required. MPC with fault detection and isolation capabilities can be applied in fault tolerant control systems. In case of detecting a faulty component in the process, the diagnostic information can be used to accommodate or reconfigure the control system to improve the availability of the process.

\section{- Eigenstructure Assignment (EA) and Pseudo-Inverse Modeling (PIM)}

The idea behind the EA method is to place the eigenvalues of a linear system using state feedback and then use any remaining degrees of freedom to align 
the eigenvectors as accurately as is possible. The eigenvalues determine the natural frequency and damping of each mode while the eigenvectors control how much each mode contributes to a given output [7]. There are several limitations to this approach when applied to reconfiguration. First, only linear systems have been considered and actuator limitations have not been taken into account. On the other hand, a perfect fault model is assumed and the effects of uncertainty have not been extensively studied.

The PIM principal is to modify the constant feedback gains so that the reconfigured system approximates the nominal system in some sense. This method uses no fault detection and isolation mechanism and certain fault models are assumed [8]. Due to significant limitations of these two methods, only a few researchers have addressed these methods.

\section{- Artificial Intelligence (AI) Methods}

The AI approaches to reconfigurable control seek to automate the expertise of the pilot and control system designer in reconfiguring the nominal control laws in case there is a failure [24]. In the case of fault diagnosis in complex systems, one is faced with the problem that no, or no sufficiently accurate, mathematical models are available. The use of knowledge-model-based techniques either in the framework of diagnosis expert systems or in combination with a human expert is then the only feasible way [13]. Neural networks and fuzzy logic approaches are powerful tools used in AI methods.

\subsubsection{Controlling the Quadrotor}

Various researchers have used the quad-rotor platform for studies in control. The quadrotor is an under-actuated system and it also requires observer style sensors (GPS, camera, ultrasound, etc.) for full attitude and position control. 
In 1922, Georges de Bothezat built a quadrotor with a rotor located at each end of a truss structure of intersecting beams, placed in the shape of a cross [5]. In time due to the tremendous improvements in manufacturing techniques and innovations in material knowledge more precise and smaller sensors can now be produced. As a result of this improvement in technology very small quadrotors are developed around the world. Some examples of the most recent and important quadrotor projects can be listed as follows:

- Dragonflyer, a commercial RC toy [25].

- Mesicopter in Stanford University [26], [27].

- Starmac in Stanford University [28].

- ANU X-4 Flyer in Australia National University [29].

- CEA X4-flyer [30]

- OS4 in Ecole Polythechnique Federal De Lausanne [1], [31].

- RAVEN in Massachusetts Institute of Technology [32].

Many researchers have also developed different control methods to stabilize the quadrotor. The work done in [33], [32], [34], [35] and [36] have used optimal Linear Quadratic Regulator (LQR) for the controller design.

Lyapunov theory is also used as another design technique as it could be found in [37], [38], [39] and [40]. According to this method, it is possible to ensure, under certain conditions, the asymptotic stability of the helicopter.

The authors in [35], [2] and [41] have used PD2 feedback and PID structures for control law design. The strength of the PD2 feedback is the exponential convergence property mainly due to the compensation of the Coriolis and gyroscopic terms. 
Backstepping and sliding mode control have been used in the work done by [42], $[43],[44],[45],[46],[47]$ and [48]. In the respective publications the convergence of the quadrotor internal states is guaranteed, however, a large number of computations is required.

A few work are also based on visual feedback. The camera used for this purpose can be mounted on-board [49], [50] and [51] (fixed on the helicopter) or off board [52] and [53] (fixed on the ground). Other control algorithms have been developed based on fuzzy techniques [54], neural networks [55], and reinforcement learning [56].

Feedback linearization method was first used by the authors in [57] to make the quadrotor track a reference trajectory. They developed the dynamic model in non linear state space representation, and used an exact linearization and noninteracting control for the global system to evaluate translational motion and yaw angle outputs.

This method was also used by the authors in [58]. In their work a PD controller was designed to control $y$ and the yaw angle, and the feedback linearization controller was implemented to control $x$ and $z$ (translational motions). In [59], feedback linearization with a high-order sliding mode observer was presented for a quadrotor and in simulation it was shown to be quite robust against wind disturbance and noise. In [46], feedback linearization method and adaptive sliding mode control for a quadrotor are presented and compared.

Among the work in the literature as reviewed briefly above, the feedback linearization technique and the LQR controller together are considered to be quite adequate for the application of our proposed fault recovery solution. The quadrotor has a nonlinear and highly coupled dynamic equations. Moreover, being an under-actuated system limits the possible choices for a proper method for control and fault recovery. As we will see in next chapters, adaptive feedback linearization is employed for the purpose of fault recovery which shows relatively an acceptable 
behavior and performance in presence of certain fault types in actuators.

\subsection{Thesis Contribution}

To the best of author's knowledge, the problem of autonomous fault recovery in under-actuated nonlinear vehicles as well as quadrotors is still an open area of research and the present work constitutes a new contribution to the problem.

A comprehensive analysis of the operation and the model of the quadrotor and actuators are provided. A control scheme is proposed to stabilize the system by taking into account the dynamics of the input moments to the system, derived from the dynamics of the actuators, which other works in the literature have not considered. A comprehensive stability analysis of the healthy system without faults is provided and investigated through simulation results. These simulations are carried out by using a model that includes relevant environmental disturbances and noise as well as parameter uncertainties.

Furthermore, different types of actuator faults are introduced and their effects on the model and performance of the system are analyzed. The solution to the autonomous fault recovery problem takes into account the management of the control authority in the system by taking advantage of the non-faulty actuators. In other words, the recovery mechanism that is proposed in this thesis does not utilize hardware redundancy (which does not exist in an under-actuated system) as the existing actuators are used to perform the required control action. The post-fault model of the faulty actuator is estimated through proper algorithms and the control laws are modified to be compatible with the post-fault system model.

In order to eliminate the need for a fault diagnosis and isolation module, the proposed fault recovery method is then extended to a general scheme which is able to monitor the performance of all the actuators and in case of fault occurrence proper 
control commands are generated to accommodate the anomalies due to the faults. The proposed fault tolerant control system is also capable of recovering in case of multiple faults in different actuators. Simulation results are presented that show successful recovery regardless of the severity of the faults. Steady state errors are also analyzed to study the performance of the proposed fault recovery method.

\subsection{Thesis Outline}

This dissertation is divided into five chapters that are described as follows. Chapter one introduces the objectives of this thesis. An overview of the motivations, the statement of the problem and a brief discussion regarding FDIR approaches and the problem of controlling the quadrotor system are made. Furthermore, the contributions of this thesis are also detailed.

The second chapter presents the mathematical model of the quadrotor and the actuators. In the first place, a simple description of the quadrotor coordinate system, physical shape and different movements are provided. The kinematics and dynamic equations that describe the quadrotor system are presented and the motor dynamics considering the gearbox and the propellers are provided.

Chapter three is dedicated to the control of the healthy quadrotor. It contains details regarding the dynamics of the input moments to the system resulting from the actuator dynamics. Comprehensive description of the methods for designing the controller are made and by taking into account some assumptions the system is partitioned into four semi-decoupled subsystems. Proper controllers are designed for each subsystem by employing LQR and feedback linearization techniques. The stability analysis of the closed-loop system is also addressed in this chapter. The final section presents the results obtained from simulations of the proposed controller.

The fourth chapter begins with a discussion on different types of faults in 
actuators followed by the modeling of these faults in the quadrotor system. Implementation of a fault recovery system is discussed in detail. The proposed adaptive feedback linearization method is then introduced, which is able to estimate the postfault parameters that are subject to change due to a fault in an actuator. Stability analysis of the closed-loop system recovered from failure is provided. The proposed adaptive controller is then extended to a fault tolerant controller that is capable of monitoring the performance of all actuators and provide proper control commands in case of faults in one or more actuators. A discussion on the types and models of faults which the system can not be recovered from by using our proposed method is also provided. Simulation results corresponding to different fault scenarios are presented in the final section of this chapter and a discussion on the performance of the fault recovery mechanism is also provided.

The last chapter lists the conclusions and the work accomplished in this thesis as well as the open problems that the author considers to be addressed and investigated in future. 


\section{Chapter 2}

\section{Mathematical Model of the}

\section{Quadrotor System}

In this chapter, the quadrotor model derivation is discussed. The importance of this chapter is due to the fact that it describes how the helicopter moves according to its inputs. Newton-Euler formulation and DC motor equations are used to model the quadrotor in this work. We will use these equations of motion to design the controller for the system in the next chapter.

In the first section 2.1 the main idea of the quadrotor dynamics is discussed. It intuitively describes which movements are allowed and how this vehicle manages to perform hovering flight. The second section 2.2 is about the mathematical derivation of the quadrotor model. In the last section 2.3, the motor dynamics considering the gearbox and the propellers are provided.

\subsection{Basic Concepts}

The quadrotor simply consists of four de motors on which propellers are mounted in a cross configuration. Each propeller is connected to the motor through the reduction gears. All the propellers axes of rotation are fixed and parallel. Furthermore, they 


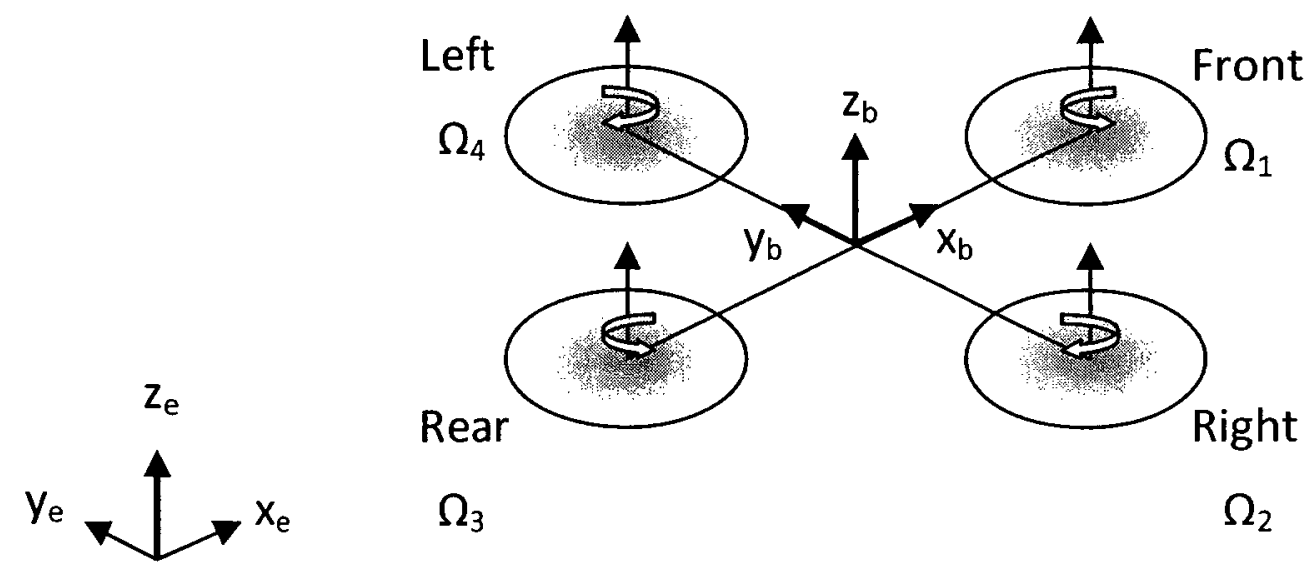

Figure 2.1: Simplified quadrotor model at hovering and coordinate systems (body and earth frame)

have fixed-pitch blades and their air flows point downward (to get an upward lift). These considerations point out that the structure is quite rigid and the only things that can vary are the propeller speeds [1].

The front and the rear propellers rotate counter-clockwise, while the left and the right ones turn clockwise. This configuration of opposite pair directions removes the need for a tail rotor (needed instead in the standard helicopter structure).

In Figure 2.1 a sketch of a simplified quadrotor structure is shown. $\Omega_{i}\left(\mathrm{rads}^{-1}\right)$ refers to the propellers rotation speed. For each propeller,two arrows are drawn: the curved one represents the direction of rotation and the straight upward arrow shows the vertical thrust force generated by the propeller. Let us consider the body fixed frame $\left(x_{B}, y_{B}, z_{B}\right)$ and earth fixed frame $\left(x_{E}, y_{E}, z_{E}\right)$ as shown in Figure 2.1.

While at hovering, all the four propellers rotate at the same speed which means that $\Omega_{i}=\Omega_{H}$ for $i=1,2,3,4$ to counterbalance the acceleration due to gravity. Although the quadrotor has $6 \mathrm{DOF}$, it is just equipped with four propellers, hence it is not possible to reach a desired set point for all the DOF and the system is underactuated. However by selecting four controllable variables properly, it is possible to 


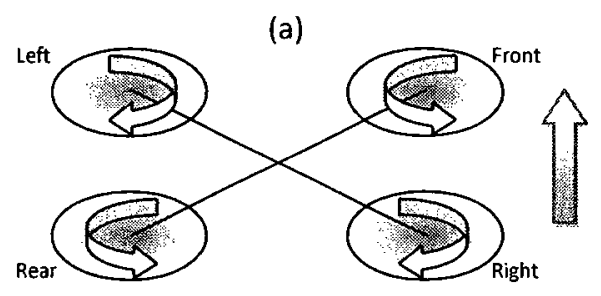

(b)
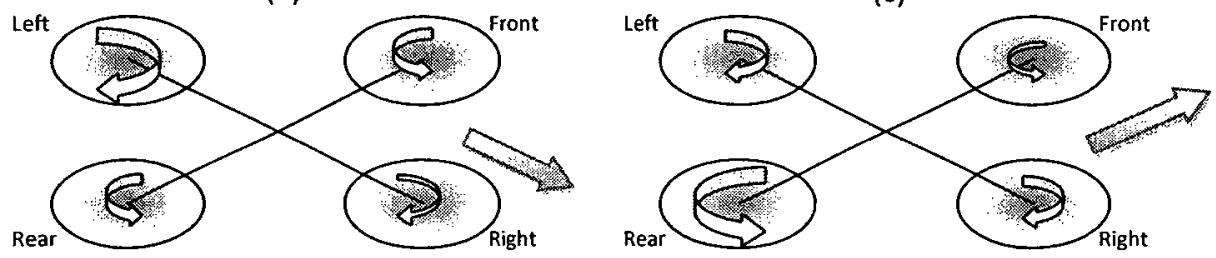

(d)
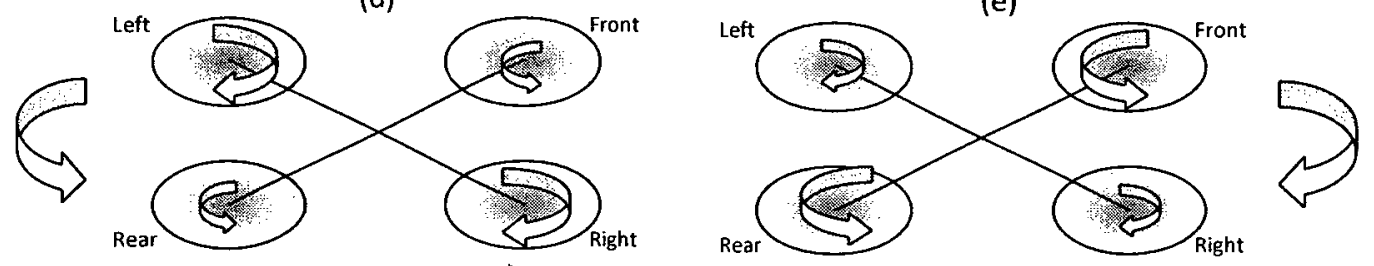

Figure 2.2: Quadrotor movement concepts; (a) Going up; (b) Moving right; (c) Moving forward; (d) Rotating left; (e) and Rotating right.

design a controller so that the vehicle could reach a desired height and attitude.

Basic movements in the quadrotor are achieved by the differences between the propellers speeds. Vertical rotation is achieved by creating an angular speed difference between the two pairs of rotors. Increasing or decreasing the speed of the four propellers simultaneously permits climbing and descending. Rotation about the longitudinal and the lateral axis and consequently horizontal motions are achieved by tilting the vehicle. This is possible by conversely changing the propeller speed of one pair of rotors as described in Figure 2.2. In this figure the width of the arrows is proportional to the propellers angular speed.

Considering $\Omega_{H}\left(\operatorname{rads}^{-1}\right)$ as the speed of the propellers at hovering condition 
and $\Omega_{1}, \Omega_{2}, \Omega_{3}$ and $\Omega_{4}$ referring to the speed of the front, right, rear and left propeller respectively, then the movements can be described as follows:

- $\Omega_{i}=\Omega_{H}+\Delta_{A}$ for $i=1,2,3,4$ leads to a vertical force $(U 1)$ with respect to body-fixed frame which raises or lowers the quadrotor. This is shown in Figure 2.2(a). $\Delta_{A}\left(\mathrm{rads}^{-1}\right)$ is a positive variable which represents an increment with respect to the constant $\Omega_{H}$.

- $\Omega_{1}=\Omega_{3}=\Omega_{H} \& \Omega_{2}=\Omega_{H}-\Delta_{A}, \Omega_{4}=\Omega_{H}+\Delta_{B}$ lead to a roll moment $\left(U_{2}\right)$ coupled with its lateral motion along the $x_{b}$ axis. The positive variables $\Delta_{A}$ and $\Delta_{B}\left(\mathrm{rads}^{-1}\right)$ are chosen to maintain the vertical thrust unchanged. This is shown in Figure 2.2(b).

- $\Omega_{1}=\Omega_{H}+\Delta_{A}, \Omega_{3}=\Omega_{H}-\Delta_{B} \& \Omega_{2}=\Omega_{4}=\Omega_{H}$ lead to a pitch moment $\left(U_{3}\right)$ coupled with lateral motion with respect to $y_{b}$ which is similar to the pitch moment but it differs in the direction of the lateral movement. which is shown in Figure 2.2(c).

- $\Omega_{1}=\Omega_{3}=\Omega_{H} \& \Omega_{2}=\Omega_{4}=\Omega_{H}+\Delta_{A}$ or $\Omega_{1}=\Omega_{3}=\Omega_{H}+\Delta_{A} \& \Omega_{2}=\Omega_{4}=\Omega_{H}$ lead to a torque $\left(U_{4}\right)$ with respect to the $z_{b}$ axis which makes the quadrotor turn left or right. The yaw movement is generated due to the fact that the left-right propellers rotate clockwise while the front-rear ones rotate counter clockwise. This is shown in Figures 2.2 (d) and (e).

\subsection{Newton-Euler Model}

This section provides a mathematical model for the quadrotor derived from NewtonEuler formulation. At the start descriptions of the kinematics and dynamics which identify a 6 DOF rigid body are presented. 


\subsubsection{Kinematics Model}

Kinematics is a branch of classical mechanics that describes the motion of objects without consideration of the causes leading to the motion. To describe a 6 DOF rigid body two reference frames are defined [60]:

- Earth inertial reference frame ( $E$-frame)

- Body-fixed reference frame ( $B$-frame)

$E$-frame $\left(x_{E}, y_{E}, z_{E}\right)$ is used to define the linear position $\Gamma^{E}(m)$ and the angular position $\Theta^{E}$ (rad) of the quadrotor. The $B$-frame $\left(x_{B}, y_{B}, z_{B}\right)$ is attached to the body. The linear velocity $V^{B}\left(m s^{-1}\right)$, the angular velocity $\omega^{B}\left(\operatorname{rads}^{-1}\right)$, the forces $F^{B}(N)$ and the torques $\tau^{B}(N m)$ are defined in this frame. The linear position $\Gamma^{E}$ is determined by the coordinates of the vector between the origin of the $B$-frame and the origin of the $E$-frame with respect to the $E$-frame as in equation (2.1)

$$
\Gamma^{E}=\left[\begin{array}{lll}
x & y & z
\end{array}\right]^{T}
$$

The angular position $\Theta^{E}$ of the quadrotor is defined by the orientation of the $B$-frame with respect to the $E$-frame which is given by three rotations about the main axes that take the $E$-frame into the $B$-frame. Euler angles (roll, pitch, yaw) are used for this purpose as in equation (2.2)

$$
\Theta^{E}=\left[\begin{array}{lll}
\phi & \theta & \psi
\end{array}\right]^{T}
$$

Considering a right-hand oriented coordinate system, the three single rotations are described separately by:

- Rotation about the x-axis of the $\phi$ angle (roll) through $R(x, \phi)$.

- Rotation about the y-axis of the $\theta$ angle (pitch) through $R(y, \theta)$. 
(a)

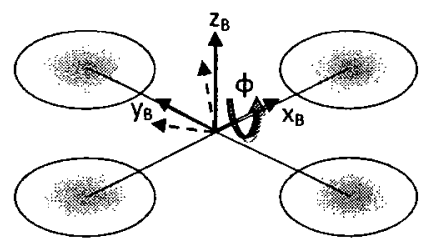

(b)

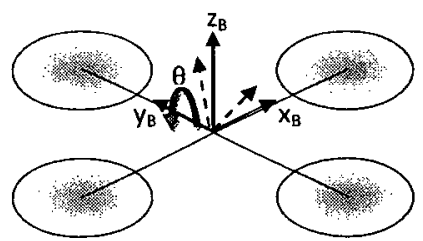

(c)

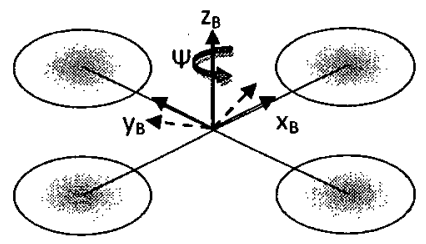

Figure 2.3: Quadrotor rotation; (a) Roll; (b) Pitch; and (c) Yaw.

- Rotation about the z-axis of the $\psi$ angle (yaw) through $R(z, \psi)$.

This is also shown in Figure 2.3. These rotation matrices are presented in [60]:

$$
\begin{aligned}
& R(x, \phi)=\left[\begin{array}{ccc}
1 & 0 & 0 \\
0 & \cos \phi & -\sin \phi \\
0 & \sin \phi & \cos \phi
\end{array}\right] \\
& R(y, \theta)=\left[\begin{array}{ccc}
\cos \theta & 0 & \sin \theta \\
0 & 1 & 0 \\
-\sin \theta & 0 & \cos \theta
\end{array}\right] \\
& R(z, \psi)=\left[\begin{array}{ccc}
\cos \psi & -\sin \psi & 0 \\
\sin \psi & \cos \psi & 0 \\
0 & 0 & 1
\end{array}\right]
\end{aligned}
$$

The complete rotation matrix $R_{\Theta}$ is obtained by multiplying these three matrices.

$$
R_{\Theta}=R(x, \phi) R(y, \theta) R(z . \psi)
$$


which results in :

$$
R_{\Theta}=\left[\begin{array}{ccc}
\cos \psi \cos \theta & \cos \psi \sin \theta \sin \phi-\sin \psi \cos \phi & \cos \phi \sin \theta \cos \phi+\sin \psi \sin \phi \\
\sin \psi \cos \theta & \sin \psi \sin \theta \sin \phi+\cos \psi \cos \phi & \sin \phi \sin \theta \cos \phi-\sin \phi \cos \psi \\
-\sin \theta & \cos \theta \sin \phi & \cos \theta \cos \phi
\end{array}\right]
$$

The linear $V^{B}$ and the angular $\omega^{B}$ velocities are defined in the $B$-frame as

$$
\begin{aligned}
V^{B} & =\left[\begin{array}{lll}
u & v & w
\end{array}\right]^{T} \\
\omega^{B} & =\left[\begin{array}{lll}
p & q & r
\end{array}\right]^{T}
\end{aligned}
$$

The relations between linear and angular positions and velocities in the $B$-frame and the $E$-frame are defined from (2.10) and(2.11) [60]:

$$
\begin{aligned}
& V^{E}=\dot{\Gamma}^{E}=R_{\ominus} \cdot V^{B} \\
& \omega^{E}=\dot{\Theta}^{E}=T_{\ominus} \cdot \omega^{B}
\end{aligned}
$$

where $T_{\Theta}$ is given by

$$
T_{\Theta}=\left[\begin{array}{ccc}
1 & 0 & -\sin \phi \\
0 & \cos \phi & \cos \theta \sin \phi \\
0 & -\sin \phi & \cos \theta \cos \phi
\end{array}\right]^{-1}
$$

For complete represent ion of the body in space we can combine linear or angular quantities and define generalized position $\xi$ and velocity $\nu$ vectors as in (2.13) and (2.14) according to

$$
\xi=\left[\begin{array}{ll}
\Gamma^{E} & \Theta^{E}
\end{array}\right]^{T}=\left[\begin{array}{llllll}
x & y & z & \phi & \theta & \psi
\end{array}\right]^{T}
$$




$$
\nu=\left[\begin{array}{ll}
V^{E} & \omega^{E}
\end{array}\right]^{T}=\left[\begin{array}{llllll}
u & v & w & p & q & r
\end{array}\right]^{T}
$$

From (2.10) and (2.11) it is possible to relate the generalized position $\xi$ in the $E$-frame with the generalized velocity $\nu$ in the $B$-frame as in equation (2.15):

$$
\begin{gathered}
\dot{\xi}=J_{\Theta} \nu \\
{\left[\begin{array}{c}
\dot{\Gamma^{E}} \\
\dot{\Theta^{E}}
\end{array}\right]=\left[\begin{array}{cc}
R_{\Theta} & 0_{3 \times 3} \\
0_{3 \times 3} & T_{\Theta}
\end{array}\right]\left[\begin{array}{c}
V^{B} \\
\omega^{B}
\end{array}\right]}
\end{gathered}
$$

\subsubsection{Dynamic Models}

Dynamics is a branch of mechanics that is concerned with the effects of forces on the motion of a body or system of bodies, especially of forces that do not originate within the system itself.

In deriving the dynamical equations of the quadrotor the following assumptions are taking into account [1], [61] :

- The structure is supposed to be fully rigid.

- The structure is considered symmetric.

- The propellers are assumed to be rigid.

- The thrust and the drag are proportional to the square of the propellers speed.

- The origin of the body fixed frame is coincident with the center of mass of the body.

- Axes of the body fixed frame coincide with the body principal axes of inertia.

Assuming that the structure is rigid and the propellers eliminate the consideration of forces acting on individual elements of the mass, while the last assumption leads to a diagonal inertia matrix $I$, simplifies the equations considerably. 
Since a rigid body has both translational and rotational motion, two independent balance laws are required to fully specify the motion of the body. As a result of Newton's second law, Euler's two laws are used to completely specify the translational (first law) and rotational (second law) motions of a rigid body [62].

Euler's first law describes how the forces control the translational motion of the rigid body. In other words, it states that the resultant force applied to a rigid body is equal to the product of the mass of the rigid body and the acceleration of the center of mass of the rigid body shown in equation (2.17):

$$
m \ddot{\Gamma}^{E}=F^{E}
$$

where $\ddot{\Gamma}^{E}\left(m s^{-2}\right)$ is the quadrotor linear acceleration vector with respect to $E$ frame, $m(K g)$ is the quadrotor mass, and $F(N)$ is the forces vector with respect to the $E$-frame. From (2.17) we can derive (2.18) where the relation in expressed in the $B$-frame

$$
\begin{gathered}
m \widehat{\dot{R_{\Theta} V^{B}}}=R_{\Theta} F^{B} \\
m\left(R_{\Theta} \dot{V}^{B}+\dot{R}_{\Theta} V^{B}\right)=R_{\Theta} F^{B}
\end{gathered}
$$

The following relation also exists for the rotation matrix $R_{\Theta}$, namely

$$
\dot{R_{\Theta}}=R_{\Theta} S(\omega)
$$

In (2.19), $S(\omega)$ is a skew-symmetric matrix such that $S(\omega) V=\omega \times V$ for any vector $V \in \mathbb{R}^{3}$, where $\times$ denotes the vector cross-product. In other words, for a given vector 
like $K=\left[\begin{array}{lll}k_{1} & k_{2} & k_{3}\end{array}\right]^{T}$ the skew-symmetric matrix $S(\omega)$ is defined as follows:

$$
S(\omega)=\left[\begin{array}{ccc}
0 & -k_{3} & k_{2} \\
k_{3} & 0 & -k_{1} \\
-k_{2} & k_{1} & 0
\end{array}\right]
$$

Using equation (2.19) one can rewrite and simplify (2.18) as in (2.21),

$$
\begin{gathered}
m R_{\Theta}\left(\dot{V}^{B}+\omega^{B} \times V^{B}\right)=R_{\Theta} F^{B} \\
m\left(\dot{V}^{B}+\omega^{B} \times V^{B}\right)=F^{B}
\end{gathered}
$$

Euler's second laws describes how the change of angular momentum of the rigid body is controlled by the moment of forces, that is

$$
I \ddot{\Theta}^{E}=\tau^{E}
$$

In $(2.22), I\left(N m s^{2}\right)$ is the body inertia matrix (in the body-fixed frame), $\ddot{\Theta}^{E}\left(\mathrm{rads}^{-2}\right)$ is the quadrotor angular acceleration vector with respect to the $E$ frame, and $\tau^{E}(N m)$ is the quadrotor torques vector with respect to the $E$-frame.

Expanding the relation in (2.22) results in (2.23) where $\dot{\omega}^{B}\left(\mathrm{rads}^{-2}\right)$ is the quadrotor angular acceleration vector with respect to the $B$-frame as

$$
I \dot{\omega}^{B}+\omega^{B} \times\left(I \omega^{B}\right)=\tau^{B}
$$

By putting equations (2.21) and (2.23) in one equation it is possible to fully describe the translational and rotational equations of motions of a 6 DOF rigid body as given 
by $(2.24)$ that is

$$
\left[\begin{array}{cc}
m . I_{3 \times 3} & 0_{3 \times 3} \\
0_{3 \times 3} & I
\end{array}\right]\left[\begin{array}{c}
\dot{V}^{B} \\
\dot{\omega}^{B}
\end{array}\right]+\left[\begin{array}{c}
\omega^{B} \times\left(m V^{B}\right) \\
\omega^{B} \times\left(I \omega^{B}\right)
\end{array}\right]=\left[\begin{array}{c}
F^{B} \\
\tau^{B}
\end{array}\right]
$$

where the notation $I_{3 \times 3}$ denotes an identity matrix with dimension $3 \times 3$.

By introducing a generalized force vector as in equation (2.25), one can rewrite the equation (2.24) in one matrix form as shown in (2.26)

$$
\begin{gathered}
\Lambda=\left[\begin{array}{ll}
F^{B} & \tau^{B}
\end{array}\right]^{T}=\left[\begin{array}{llllll}
F_{x} & F_{y} & F_{z} & \tau_{x} & \tau_{y} & \tau_{z}
\end{array}\right]^{T} \\
M_{B} \dot{\nu}+C_{B}(\nu) \nu=\Lambda
\end{gathered}
$$

where $\dot{\nu}$ is the generalized acceleration vector with respect to the $B$-frame. The $M_{B}[+]$ and $C_{B}(\nu)$ matrices are defined as in equations (2.27) and (2.28) by

$$
M_{B}=\left[\begin{array}{cc}
m . I_{3 \times 3} & 0_{3 \times 3} \\
0_{3 \times 3} & I
\end{array}\right]=\left[\begin{array}{cccccc}
m & 0 & 0 & 0 & 0 & 0 \\
0 & m & 0 & 0 & 0 & 0 \\
0 & 0 & m & 0 & 0 & 0 \\
0 & 0 & 0 & I_{x x} & 0 & 0 \\
0 & 0 & 0 & 0 & I_{y y} & 0 \\
0 & 0 & 0 & 0 & 0 & I_{z z}
\end{array}\right]
$$




$$
C_{B}(\nu)=\left[\begin{array}{cc}
0_{3 \times 3} & -m S\left(v^{B}\right) \\
0_{3 \times 3} & -S\left(I \omega^{B}\right)
\end{array}\right]=\left[\begin{array}{cccccc}
0 & 0 & 0 & 0 & m w & -m v \\
0 & 0 & 0 & -m w & 0 & m u \\
0 & 0 & 0 & m v & -m u & 0 \\
0 & 0 & 0 & 0 & I_{z z} r & -I_{y y} q \\
0 & 0 & 0 & -I_{z z} r & 0 & I_{x x} p \\
0 & 0 & 0 & -I_{y y} q & -I_{x x} p & 0
\end{array}\right]
$$

Equation (2.26) is a general equation for any rigid body provided that the considered assumptions are valid. The forces that are effective on the quadrotor body can be divided to three components as

- The force due to gravity.

- The torque due to the effect produced by the propeller rotation.

- The thrust force and body moments due to the forces generated by rotors.

The gravitational vector is given by (2.29) due to the acceleration from the gravity $g\left(\mathrm{~m} \mathrm{~s}^{-2}\right)$. This force is about the $z$-axis and is only effective on the linear and not the angular equations

$$
G_{B}(\xi)=\left[\begin{array}{c}
F_{G}^{B} \\
0_{3 \times 1}
\end{array}\right]=\left[\begin{array}{c}
R_{\Theta}^{-1} F_{G}^{E} \\
0_{3 \times 1}
\end{array}\right]=\left[R_{\Theta}^{T}\left[\begin{array}{c}
0 \\
0 \\
-m g
\end{array}\right]=\left[\begin{array}{c}
m g \sin \theta \\
-m g \cos \theta \sin \phi \\
-m g \cos \theta \sin \phi \\
0 \\
0 \\
0
\end{array}\right]\right.
$$

where $F_{G}^{B}(N)$ is the gravitational force vector with respect to the $B$-frame and 
$F_{G}^{E}(N)$ is the one with respect to the $E$-frame. It is to be noted that $R_{\Theta}$ is an orthogonal normalized matrix and its inverse is equal to its transpose.

The quadrotor also experiences a torque due to the gyroscopic effects produced by the propellers according to equation (2.30) [4]. Since two propellers rotate clockwise and the other pair rotates counter clockwise, there is an overall imbalance if the algebraic sum of the rotor speeds is not zero. The axes of these propellers (spin axes) are parallel to $z$-axis of the platform. When the quadrotor rolls or pitches it changes the direction of the angular momentum vectors of the four motors. The result is a gyroscopic torque that attempts to turn the spin axis so that it aligns with rotation around the $z$-axis, that is

$$
\begin{aligned}
& O_{B}(\nu)=\left[-\sum_{k=1}^{4} J_{t p}\left(\omega^{0_{3 \times 1}} \times\left[\begin{array}{c}
0 \\
0 \\
1
\end{array}\right]\right)=\left[\begin{array}{c}
0_{3 \times 1} \\
(-1)^{k} \Omega_{k}
\end{array}\right]\right. \\
& =J_{t p}\left[\begin{array}{cccc}
0 & 0 & 0 & 0 \\
0 & 0 & 0 & 0 \\
0 & 0 & 0 & 0 \\
q & -q & q & -q \\
-p & p & -p & p \\
0 & 0 & 0 & 0
\end{array}\right] \Omega
\end{aligned}
$$

In the above equation $O_{B}(\nu)$ is the gyroscopic propeller matrix and $J_{t p}\left(N m s^{2}\right)$ is the total rotational moment of inertia around the propeller axis which will be discussed in the next section. The variables $\Omega_{T}\left(\mathrm{rads}^{-1}\right)$ and $\Omega\left(\mathrm{rads}^{-1}\right)$ are the overall propellers' speed and the propellers' speed vector, respectively, as defined in equation (2.31) 


$$
\Omega_{T}=-\Omega_{1}+\Omega_{2}-\Omega_{3}+\Omega_{4} ; \quad \Omega=\left[\begin{array}{c}
\Omega_{1} \\
\Omega_{2} \\
\Omega_{3} \\
\Omega_{4}
\end{array}\right]
$$

A voltage applied to each motor results in a net torque being applied to the rotor shaft, which results in a thrust $T_{i}$. Forward velocity also causes a drag force on the rotor that acts opposite to the direction of travel $D_{i}$. As stated in the assumptions in the beginning of this section, the thrust and the drag are proportional to the square of each propellers speed as given by (2.32)

$$
T_{i}=b \Omega_{i}^{2} \text { and } D_{i}=d \Omega_{i}^{2}
$$

In equation (2.32) $b$ and $d$ denote the thrust and the drag coefficients, respectively.

The thrust force and the body moments effective on the quadrotor due to the combination of the rotors rotation are modeled by equation (2.33) as a movement vector $U_{B}(\Omega)$, that is

$$
U_{B}(\Omega)=E_{B} \Omega^{2}=\left[\begin{array}{c}
0 \\
0 \\
U_{1} \\
U_{2} \\
U_{3} \\
U_{4}
\end{array}\right]=\left[\begin{array}{c}
0 \\
0 \\
b\left(\Omega_{1}^{2}+\Omega_{2}^{2}+\Omega_{3}^{2}+\Omega_{4}^{2}+\right) \\
l b\left(\Omega_{4}^{2}-\Omega_{2}^{2}\right) \\
l b\left(\Omega_{3}^{2}-\Omega_{1}^{2}\right) \\
d\left(-\Omega_{1}^{2}+\Omega_{2}^{2}-\Omega_{3}^{2}+\Omega_{4}^{2}\right)
\end{array}\right]
$$

where $l(m)$ is the distance between the center of the quadrotor and the center of a propeller, $U_{1}, U_{2}, U_{3}$ and $U_{4}$ are the movement vector components introduced in the previous section and $E_{B}$ is also a constant matrix defined in (2.34), that is. 


$$
E_{B}=\left[\begin{array}{cccc}
0 & 0 & 0 & 0 \\
0 & 0 & 0 & 0 \\
b & b & b & b \\
0 & -l b & 0 & l b \\
-l b & 0 & l b & 0 \\
-d & d & -d & d
\end{array}\right]
$$

Considering the above mentioned forces and torques in (2.29),(2.30) and (2.33) we can rewrite equation (2.26) to fully describe the dynamics of the quadrotor vehicle as given by equation (2.35), that is

$$
M_{B} \dot{\nu}+C_{B}(\nu) \nu=G_{B}(\xi)+O_{B}(\nu)+E_{B} \Omega^{2}
$$

By rearranging equation (2.35) it is possible to isolate the derivate of the generalized velocity vector with respect to the $B$-frame $\dot{\nu}$, according to

$$
\dot{\nu}=M_{B}^{-1}\left(-C_{B}(\nu) n u+G_{B}(\xi)+O_{B}(\nu)+E_{B} \Omega^{2}\right)
$$

Equation (2.37) shows the previous expression as a system of equations, namely.

$$
\left\{\begin{array}{l}
\dot{u}=(v r-w q)+g \sin \theta \\
\dot{v}=(w p-u r)-g \cos \theta \sin \phi \\
\dot{w}=(u q-v p)-g \cos \theta \sin \phi+\frac{U_{1}}{m} \\
\dot{p}=\frac{I_{y y}-I_{z z}}{I_{x x}} q r-\frac{J_{t p}}{I_{x x}} q \Omega_{T}+\frac{U_{2}}{I_{x x}} \\
\dot{q}=\frac{I_{z z}-I_{x x}}{I_{y y}} p r+\frac{I_{t p}}{I_{y y}} p \Omega_{T}+\frac{U_{3}}{I_{y y}} \\
\dot{r}=\frac{I_{x x}-I_{y y}}{I_{z z}} q r+\frac{U_{4}}{I_{z z}}
\end{array}\right.
$$


where the propellers' speed inputs are given through the equation (2.38)

$$
\left\{\begin{array}{l}
U_{1}=b\left(\Omega_{1}^{2}+\Omega_{2}^{2}+\Omega_{3}^{2}+\Omega_{4}^{2}\right) \\
U_{2}=l b\left(\Omega_{4}^{2}-\Omega_{2}^{2}\right) \\
U_{3}=l b\left(\Omega_{3}^{2}-\Omega_{1}^{2}\right) \\
U_{4}=d\left(-\Omega_{1}^{2}+\Omega_{2}^{2}-\Omega_{3}^{2}+\Omega_{4}^{2}\right) \\
\Omega_{T}=-\Omega_{1}+\Omega_{2}-\Omega_{3}+\Omega_{4}
\end{array}\right.
$$

The quadrotor dynamics in equation (2.37) is written in the $B$-frame. It is more useful and practical for control purposes to write the dynamic equations in the $E$-frame and for the generalized position vector as defined in (2.13). For simplicity of the equations it is possible to assume that the angular velocity in the $E$-frame $\dot{\Theta}^{E}$ is equal to the angular velocity in the $B$-frame $\omega^{B}$. This assumption can be verified through the fact that in hovering condition the transfer matrix $T_{\Theta}$, as defined in (2.12), is close to an identity matrix.

By using the relation defined in (2.15) and assuming $T_{\Theta}=I_{3 \times 3}$ in (2.16), it is possible to map the system dynamics in (2.37) from the $B$-frame to the $E$-frame as given by equation (2.39)

$$
\left\{\begin{array}{l}
\ddot{x}=(\cos \phi \sin \theta \cos \psi+\sin \phi \sin \psi) \frac{U_{1}}{m} \\
\ddot{y}=(\cos \phi \sin \theta \sin \psi-\sin \phi \cos \psi) \frac{U_{1}}{m} \\
\ddot{z}=-g+(\cos \phi \cos \theta) \frac{U_{1}}{m} \\
\ddot{\phi}=\dot{\theta} \dot{\phi}\left(\frac{I_{y y}-I_{z z}}{I_{x x}}\right)-\frac{J_{t p}}{I_{x x}} \dot{\theta} \Omega_{T}+\frac{U_{2}}{I_{x x}} \\
\ddot{\theta}=\dot{\phi} \dot{\psi}\left(\frac{I_{z z}-I_{x x}}{I_{y y}}\right)+\frac{J_{t p}}{I_{y y}} \dot{\phi} \Omega_{T}+\frac{U_{3}}{I_{y y}} \\
\ddot{\psi}=\dot{\phi} \dot{\theta}\left(\frac{I_{x x}-I_{y y}}{I_{z z}}\right)+\frac{U_{4}}{I_{z z}}
\end{array}\right.
$$

where the propellers' speed inputs are the same as in the system with respect to the 


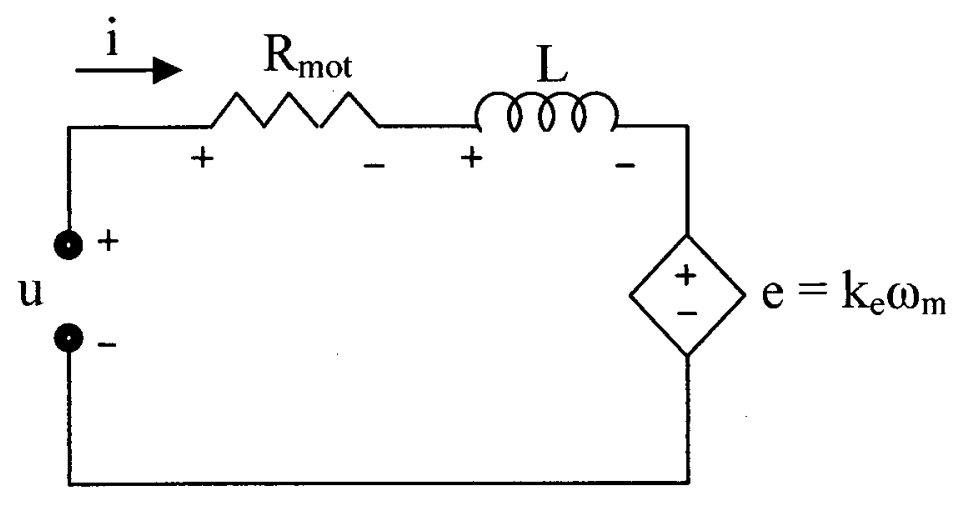

Figure 2.4: DC motor model

$B$-frame and given through the equation (2.38).

\subsection{The DC Motor Actuator}

The DC motor is an electromechanical device with an electrical input (voltage, current) and a mechanical output (speed, torque). Operating as an actuator, it converts the electrical energy into the mechanical energy.

The DC motor basically consists of a fixed stator and a rotor which is free to rotate around the stator. The characteristics of a DC motor can be analyzed through a well-known model which links both electrical and mechanical quantities.

The model consists of three elements in series for the stator as shown in Figure 2.4. It is characterized by an inductance $L$, due to the windings and a resistance $R_{m o t}$, due to the dispersions in the conductor. The input to the motor is the voltage $u(V)$ and the generator $e$, called as the back-EMF, also supplies a voltage proportional to the motor speed.

Applying the KCL to the circuit diagram of Figure 2.4 results in 


$$
u=R_{m o t} i+L \frac{d i}{d t}+e
$$

where

$$
e=k_{e} \omega_{m}
$$

In equation (2.41), $k_{e}\left(V s \mathrm{rad}^{-1}\right)$ is called the back-EMF constant and $\omega_{m}\left(\mathrm{rad} \mathrm{s}^{-1}\right)$ is the motor angular speed. Most of the motors used in robotics have small and negligible inductance due to the construction optimization [61]. For more simplicity and having a first order dynamic equation instead of a second order one, the effect of inductance could be neglected. With this assumption equations (2.40)-(2.41) are simplified to

$$
u=R_{m o t} i+k_{e} \omega_{m}
$$

The dynamics of the motor is described by the following equation

$$
J_{t m} \dot{\omega}_{m}=M_{t}-M_{l}
$$

where $J_{t m}\left(N m s^{2}\right)$ is the total motor moment of inertia, $\dot{\omega}_{m}\left(\mathrm{rads}^{-2}\right)$ is the motor angular acceleration, $M_{t}(N \mathrm{~m})$ is the motor torque, and $M_{l}(N \mathrm{~m})$ is the load torque. The torque produced in the motor is proportional to the current $i$ and is given by (2.44) where $k_{t}\left(N m A^{-1}\right)$ is called the torque constant, that is

$$
M_{t}=k_{t} i
$$

It is to be noted that the torque constant $k_{t}\left(N m A^{-1}\right)$ has the same numerical value as the back-EMF constant $k_{e}\left(V s \mathrm{rad}^{-1}\right)$. This is verified through the fact that the mechanical and electrical power are equal in the motor.

From equations (2.42) and (2.44), the dynamic equation in (2.43) is rewritten 
as in (2.45), namely

$$
\begin{aligned}
J_{t m} \dot{\omega}_{m} & =k_{t} \frac{u-k_{e} \omega_{m}}{R_{m o t}}-M_{l} \\
& =-\frac{k_{e}^{2}}{R_{m o t}} \omega_{m}-M_{l}+\frac{k_{e}}{R_{m o l}} u
\end{aligned}
$$

The real motor system is composed of the motor itself, the gear box and the propeller. The gear box has a reduction ratio $r$, which is equal to the motor speed $\omega_{m}$, divided by the propeller speed $\omega_{p}$. It also has an efficiency factor $\eta$ which binds the mechanical power of the motor axis $P_{m}$ to the propeller axis one $P_{p}\left(N m s^{-1}\right)$, that is

$$
\left\{\begin{array}{l}
r=\frac{\omega_{m}}{\omega_{p}} \\
\eta=\frac{P_{p}}{P_{m}}
\end{array}\right.
$$

Considering the propeller and the gearbox, the load torque experienced by the motor is given by equation (2.47), that is

$$
M_{l}=\frac{d}{\eta r^{3}} \omega_{m}^{2}
$$

where $d\left(N m s^{2}\right)$ is the aerodynamic drag factor. The total inertia seen by the motor can also be described as

$$
J_{t m}=J_{m}+\frac{J_{p}}{\eta r^{2}}
$$

where $J_{m}\left(N m s^{2}\right)$ is the rotor moment of inertia about the motor axis and $J_{p}\left(N m s^{2}\right)$ is the rotor moment of inertia about the propeller axis.

Equation (2.45) could be rewritten according to (2.46) and (2.48) as follows

$$
\left(J_{m}+\frac{J_{p}}{\eta r^{2}}\right) \dot{\omega}_{m}=-\frac{k_{e}^{2}}{R_{m o t}} \omega_{m}-\frac{d}{\eta r^{3}} \omega_{m}^{2}+\frac{k_{e}}{R_{m o t}} u
$$

Equation (2.49) is formulated with respect to the motor axis. It is possible to 
reformulate this equation from the propeller axis as shown below (2.50).

$$
\left(J_{p}+\eta r^{2} J_{m}\right) \dot{\omega}_{p}=-\frac{k_{e}^{2}}{R_{m o t}} \eta r^{2} \omega_{p}-d \omega_{p}^{2}+\frac{k_{e}}{R_{m o t}} \eta r u
$$

From equation (2.50), the total rotational inertia around the propeller axis $J_{t p}\left(N m s^{2}\right)$ can be defined as follows:

$$
J_{t p}=\left(J_{p}+\eta r^{2} J_{m}\right)
$$

By setting $\frac{k_{\epsilon}^{2}}{R_{\text {mot }} J_{t p}} \eta r^{2}=\frac{1}{\tau_{t}}$, the equation (2.50) could be rewritten as:

$$
\dot{\omega}_{p}=-\frac{1}{\tau_{t}} \omega_{p}-d \omega_{p}^{2}+\frac{1}{k_{e} r \tau_{t}} u
$$

The above dynamic equation of the motor system will be used in the next chapter to design a controller for the quadrotor vehicle.

\subsection{Conclusions}

In this chapter the fundamental concepts on the quadrotor motion and dynamics are introduced. Furthermore, the description of the coordinate systems, kinematics and dynamic models and the Newton-Euler formulation of the system are presented. The model of the actuators of the quadrotor are also discussed and included in the entire model of the system to obtain a complete representation of the quadrotor system.

The next chapter will be devoted to designing a controller for this system to meet the goal of tracking a given reference trajectory without any faults. 


\section{Chapter 3}

\section{Control Design for the Healthy}

\section{Quadrotor System}

In this chapter a controller is designed to stabilize the quadrotor. As mentioned in Chapter 2, quadrotor has a nonlinear and a highly coupled dynamics with 6 DOF and four inputs which makes it an under-actuated system. The Feedback Linearization (FL) and Linear Quadratic Regulator (LQR) techniques will be used in this chapter for designing the controller.

FL technique is known as a powerful method for control of nonlinear systems. It has been used in both the control and the fault recovery mechanism of systems. One of the important reasons for choosing this method over the other existing control methods is the fact that we are considering the actuator dynamics in controlling the system. In FL technique these dynamics are taking into account directly and therefore in case of fault occurrence, the system is able to accommodate the fault by changing the feedback law adaptively to reduce the fault effects on the system. The LQR technique is also used along the FL method to meet the goal of tracking a desired trajectory optimally.

The first section (3.1) discusses the basic simplifications that are assumed 
in the quadrotor model that is developed in Chapter 2 for control design. These simplifications will reduce the complexity of the control algorithm.

The second section (3.2) derives the dynamics of the input moments to the quadrotor from the dynamics of the rotors. By using a simple transform it is possible to decouple the dynamic equations to simplify the design of the controller.

In the third section (3.3) the controllers that are designed for the quadrotor by using the FL and the LQR techniques are presented.

The last section of this chapter presents the simulation results for the closed loop system of the quadrotor.

\subsection{Control Modeling}

The dynamics of the quadrotor is well described in the previous chapter. The most important concepts can be summarized in equations (3.1), (3.2) and (3.3). The first equation shows how the quadrotor accelerates according to the basic movement commands given. It should be noted that the following equations in the $E$-frame are derived by assuming that the rotation matrix $T_{\Theta}$ defined in (2.10) is close to an identity matrix, that is

$$
\left\{\begin{array}{l}
\ddot{x}=(\cos \phi \sin \theta \cos \psi+\sin \phi \sin \psi) \frac{U_{1}}{m} \\
\ddot{y}=(\cos \phi \sin \theta \sin \psi-\sin \phi \cos \psi) \frac{U_{1}}{m} \\
\ddot{z}=-g+(\cos \phi \cos \theta) \frac{U_{1}}{m} \\
\ddot{\phi}=\dot{\theta} \dot{\phi}\left(\frac{I_{y y}-I_{z z}}{I_{x x}}\right)-\frac{J_{t p}}{I_{x x}} \dot{\theta} \Omega_{T}+\frac{U_{2}}{I_{x x}} \\
\ddot{\theta}=\dot{\phi} \dot{\psi}\left(\frac{I_{z z}-I_{x x}}{I_{y y}}\right)+\frac{J_{t p}}{I_{y y}} \dot{\phi} \Omega_{T}+\frac{U_{3}}{I_{y y}} \\
\ddot{\psi}=\dot{\phi} \dot{\theta}\left(\frac{I_{x x}-I_{y y}}{I_{z z}}\right)+\frac{U_{4}}{I_{z z}}
\end{array}\right.
$$

The second system of equations explains how the basic movements are related 
to the propellers' speed, namely

$$
\left\{\begin{array}{l}
U_{1}=b\left(\Omega_{1}^{2}+\Omega_{2}^{2}+\Omega_{3}^{2}+\Omega_{4}^{2}\right) \\
U_{2}=l b\left(\Omega_{4}^{2}-\Omega_{2}^{2}\right) \\
U_{3}=l b\left(\Omega_{3}^{2}-\Omega_{1}^{2}\right) \\
U_{4}=d\left(-\Omega_{1}^{2}+\Omega_{2}^{2}-\Omega_{3}^{2}+\Omega_{4}^{2}\right) \\
\Omega_{T}=-\Omega_{1}+\Omega_{2}-\Omega_{3}+\Omega_{4}
\end{array}\right.
$$

The third equation takes into accounts the motors dynamics and shows the relation between propellers' speed and motors' voltage, that is

$$
\dot{\Omega}_{i}=-\frac{1}{\tau_{t}} \Omega_{i}-d \Omega_{i}^{2}+\frac{1}{k_{e} r \tau_{t}} u
$$

Since the motion of the quadrotor can be assumed close to the hovering condition, small angular changes occur (especially for the roll and the pitch). Since the rates of change in $\theta$ and $\phi$ are small, the terms due to the gyroscopic effects appearing in the dynamic equations of $\ddot{\phi}$ and $\ddot{\theta}$ in (3.1) are also negligible. These assumptions are also verified through simulation results.

Moreover, since the structure of the quadrotor is symmetric, the body moments of inertia $I_{x x}$ and $I_{y y}$ are equal. This fact will also simplify the dynamic equation of $\ddot{\psi}$ in (3.1).

Considering the above mentioned facts, equations (3.1) and (3.2) can be rewritten as in (3.4)and (3.5), respectively, that is 


$$
\left\{\begin{array}{l}
\ddot{\ddot{x}}=(\cos \phi \sin \theta \cos \psi+\sin \phi \sin \psi) \frac{U_{1}}{m} \\
\ddot{y}=(\cos \phi \sin \theta \sin \psi-\sin \phi \cos \psi) \frac{U_{1}}{m} \\
\ddot{z}=-g+(\cos \phi \cos \theta) \frac{U_{1}}{m} \\
\ddot{\phi}=\frac{U_{2}}{I_{x x}} \\
\ddot{\theta}=\frac{U_{3}}{I_{y y}} \\
\ddot{\psi}=\frac{U_{4}}{I_{z z}} \\
\left\{\begin{array}{l}
U_{1}=b\left(\Omega_{1}^{2}+\Omega_{2}^{2}+\Omega_{3}^{2}+\Omega_{4}^{2}\right) \\
U_{2}=l b\left(\Omega_{4}^{2}-\Omega_{2}^{2}\right) \\
U_{3}=l b\left(\Omega_{3}^{2}-\Omega_{1}^{2}\right) \\
U_{4}=d\left(-\Omega_{1}^{2}+\Omega_{2}^{2}-\Omega_{3}^{2}+\Omega_{4}^{2}\right)
\end{array}\right.
\end{array}\right.
$$

The control algorithm to be designed is used to give the appropriate signals to the actuators. Since there are only four propellers, no more than four variables can be controlled in the loop. It is possible to define the position of the quadrotor in space completely by the linear position $\Gamma^{E}=\left[\begin{array}{lll}x & y & z\end{array}\right]^{T}$ and the yaw angle (heading angle) $\psi$. These four variables are indeed selected for control purposes in this work.

\subsection{Moment Dynamics}

The dynamic equations of the propeller angular speeds $\Omega_{i}\left(\mathrm{rads}^{-1}\right)$ were defined according to equation (3.3). On the other hand, the input moments to the quadrotor system that are defined in equation (3.5) are related to the propeller's speed. Hence, it is possible to derive these input moment dynamic equations and complete the quadrotor model by considering the effects of the motor dynamics on the dynamics 
of the entire system.

In Chapter 2, the thrust and the drag forces were introduced according to

$$
T_{i}=b \Omega_{i}^{2} \quad \text { and } \quad \mathrm{D}_{\mathrm{i}}=\mathrm{d} \Omega_{\mathrm{i}}^{2} \quad \mathrm{i}=1, \ldots, 4
$$

where $\Omega_{i}\left(\mathrm{rads}^{-1}\right)$ is the speed of the the $i^{\text {th }}$ propeller. It is clear that $D_{i}=\frac{d}{b} T_{i}$. From equations (3.6) and (3.3) the input voltage to the propeller $i, u_{i}$, and to the thrust $T_{i}$ dynamic equation could be obtained as follows:

$$
\begin{aligned}
\dot{T}_{i} & =2 b \Omega_{i} \dot{\Omega}_{i} \\
& =-\frac{2 b}{\tau_{t}} \Omega_{i}^{2}-2 d b \Omega_{i}^{3}+\frac{2 b \Omega_{i}}{k_{e} r \tau_{t}} u_{i} \\
& =-\frac{2}{\tau_{t}} T_{i}-\frac{2 d}{\sqrt{b}} T_{i} \sqrt{T_{i}}+\frac{2 \sqrt{b}}{k_{e} r \tau_{t}} \sqrt{T_{i}} u_{i}
\end{aligned}
$$

Since the differential equation (3.7) is nonlinear, a suitable approach is to linearize this dynamics around an operating working point, $T_{0}$. The first order Taylor series expansion has been chosen here to derive the linearized model given by

$$
\dot{T}_{i}=A_{t} T_{i}+B_{t} u_{i}+C_{t}
$$

In the above equation, the parameters $A_{t}, B_{t}$ and $C_{t}$ are the linearized coefficients and are defined as follows:

$$
\left\{\begin{array}{l}
A_{t}=\frac{-2}{\tau_{t}}-\frac{3 d}{\sqrt{b}} \sqrt{T_{0}} \\
B_{t}=\frac{\sqrt{b}}{k_{e} r \tau_{\iota}} \sqrt{T_{0}} \\
C_{t}=\frac{d}{\sqrt{b}} T_{0}^{\frac{3}{2}}
\end{array}\right.
$$

The set point corresponding to the linearizing condition could be calculated from the fact that at hovering the total thrust should be equal to the gravitational 
force effective on the quadrotor. In other words, we have

$$
\sum_{i=1}^{4} T_{i}=m g
$$

It is possible to rewrite equation (3.5) in terms of $T_{i}$ for $i=1, \ldots, 4$ as in equation (3.11), that is

$$
\left\{\begin{array}{l}
U_{1}=T_{1}+T_{2}+T_{3}+T_{4} \\
U_{2}=-l T_{2}+l T_{4} \\
U_{3}=-l T_{1}+l T_{3} \\
U_{4}=-\frac{d}{b} T_{1}+\frac{d}{b} T_{2}-\frac{d}{b} T_{3}+\frac{d}{b} T_{4}
\end{array}\right.
$$

The above equation can also be represented in a matrix form as

$$
U=L_{U T} T
$$

where $U=\left[\begin{array}{llll}U_{1} & U_{2} & U_{3} & U_{4}\end{array}\right]^{T}$ is the movement vector and $T=\left[\begin{array}{llll}T_{1} & T_{2} & T_{3} & T_{4}\end{array}\right]^{T}$ is the thrust vector. The constant matrix $L_{U T}$ is defined from

$$
L_{U T}=\left[\begin{array}{cccc}
1 & 1 & 1 & 1 \\
0 & -l & 0 & l \\
-l & 0 & l & 0 \\
-\frac{d}{b} & \frac{d}{b} & -\frac{d}{b} & \frac{d}{b}
\end{array}\right]
$$

Using the linearized dynamic equation for the thrust $T_{i}$ developed in (3.8), it is possible to find the dynamic equations from the input voltage to the propellers to the movement moments. For this purpose it is useful to write the dynamic equation 
in (3.8) in a matrix form for the thrust vector $T=\left[\begin{array}{llll}T_{1} & T_{2} & T_{3} & T_{4}\end{array}\right]^{T}$, as follows

$$
\dot{T}=-A_{T} T+B_{T} \underline{u}+C_{T}
$$

where $A_{T}=A_{t} I_{(4 \times 4)}$ and $B_{T}=B_{t} I_{(4 \times 4)}$ are constant matrices and $C_{T}=C_{t}\left[\begin{array}{llll}1 & 1 & 1 & 1\end{array}\right]^{T}$ is a constant vector. The notation $I_{(4 \times 4)}$ refers to an identity matrix of the dimension 4 , the variable $\underline{u}=\left[\begin{array}{llll}u_{1} & u_{2} & u_{3} & u_{4}\end{array}\right]^{T}$ is also defined as the vector of the input voltages to the propellers.

By left-multiplying the transfer matrix between $U$ and $T, L_{U T}$ to (3.14), the following equation is obtained

$$
L_{U T} \dot{T}=-\left(L_{U T} A_{T}\right) T+\left(L_{U T} B_{T}\right) \underline{u}+\left(L_{U T} C_{T}\right)
$$

It should be noted that:

$$
\left\{\begin{array}{l}
L_{U T} A_{T}=L_{U T}\left(A_{t} I_{(4 \times 4)}\right)=A_{t}\left(I_{(4 \times 4)}\right) L_{T U}=A_{T} L_{U T} \\
L_{U T} B_{T}=B_{T} L_{U T}
\end{array} .\right.
$$

From equation (3.12), it is possible to rewrite the equation (3.15) as

$$
\dot{U}=-A_{T} U+B_{T} \underline{v}+D_{T}
$$

In the above equation the dynamics corresponding to the movement vector are obtained. The new input vector $\underline{v}=\left[\begin{array}{llll}v_{1} & v_{2} & v_{3} & v_{4}\end{array}\right]^{T}$ is defined according to equation (3.17) as a linear transfer by $L_{U T}$ from the actual input voltage vector $\underline{u}$, 
that is

$$
\left[\begin{array}{l}
v_{1} \\
v_{2} \\
v_{3} \\
v_{4}
\end{array}\right]=\left[\begin{array}{cccc}
1 & 1 & 1 & 1 \\
0 & -l & 0 & l \\
-l & 0 & l & 0 \\
-\frac{d}{b} & \frac{d}{b} & -\frac{d}{b} & \frac{d}{b}
\end{array}\right]\left[\begin{array}{l}
u_{1} \\
u_{2} \\
u_{3} \\
u_{4}
\end{array}\right]
$$

The constant vector $D_{T}$ is also defined as follows

$$
D_{T}=L_{U T} C_{T}=\left[\begin{array}{c}
4 C_{t} \\
0 \\
0 \\
0
\end{array}\right]
$$

The system of equations shown below represent the dynamics of the movement vector as in (3.16) but now in a non-compact form, namely

$$
\left\{\begin{array}{l}
\dot{U}_{1}=A_{t} U_{1}+B_{t} v_{1}+4 C_{t} \\
\dot{U_{2}}=A_{t} U_{2}+B_{t} v_{2} \\
\dot{U}_{3}=A_{t} U_{3}+B_{t} v_{3} \\
\dot{U}_{4}=A_{t} U_{4}+B_{t} v_{4}
\end{array}\right.
$$

The purpose of the controller design is to find the proper inputs to the propellers that guarantee the system stability and desired closed-loop performance. By considering the new input vector $\underline{v}$ as the virtual input to the system and designing the controller to get the proper virtual input signal, it is possible to find the actual input voltages through the inverse of the transfer matrix $L_{U T}$ as in (3.20), that is

$$
\underline{u}=\left(L_{U T}\right)^{-1} \underline{v}
$$


By putting together the system dynamics developed in (3.4) and the moment dynamics in (3.19) the system of equations in (3.21) fully describe the dynamics of the quadrotor. It is to be noted that the rotor dynamics are taken into account in this representation of the system through the moment dynamics, namely

$$
\left\{\begin{array}{l}
\ddot{x}=(\cos \phi \sin \theta \cos \psi+\sin \phi \sin \psi) \frac{U_{1}}{m} \\
\ddot{y}=(\cos \phi \sin \theta \sin \psi-\sin \phi \cos \psi) \frac{U_{1}}{m} \\
\ddot{z}=-g+(\cos \phi \cos \theta) \frac{U_{1}}{m} \\
\ddot{\phi}=\frac{U_{2}}{I_{x x}} \\
\ddot{\theta}=\frac{U_{3}}{I_{x x}} \\
\ddot{\psi}=\frac{U_{4}}{I_{z z}} \\
\dot{U_{1}}=A_{t} U_{1}+B_{t} v_{1}+4 C_{t} \\
\dot{U}_{2}=A_{t} U_{2}+B_{t} v_{2} \\
\dot{U_{3}}=A_{t} U_{3}+B_{t} v_{3} \\
\dot{U}_{4}=A_{t} U_{4}+B_{t} v_{4}
\end{array}\right.
$$

\subsection{Development of Control Schemes for the Healthy Quadrotor}

The first part of this section provides an overview on the Linear Quadratic Regulator (LQR) [63] and Feedback Linearization (FL) [64], [65], [17] techniques in controlling a system. Moreover, the designed controller for the quadrotor system is also discussed. 


\subsubsection{LQR Controller}

The general equations for representing a linear time invariant system in state space form is shown as

$$
\left\{\begin{array}{l}
\dot{x}(t)=A x(t)+B u(t) \\
y(t)=C x(t)
\end{array}\right.
$$

where $u(t) \in \mathbb{R}, y(t) \in \mathbb{R}$ are the input and output variables, respectively, $x(t) \in \mathbb{R}^{n}$ is the state of the system and $A_{n \times n}, B_{n \times 1}$ and $C_{1 \times n}$ are the matrices defining the system. The purpose of LQR design is to find an optimal state feedback control law $K_{c}$ given in (3.23) that minimizes the cost function $J$ defined in (3.24) that is

$$
\begin{gathered}
u(t)=-K_{c} x(t) \\
J=\int\left(x^{T} Q x+u^{T} R u\right) d t
\end{gathered}
$$

where $Q$ and $R$ are symmetric positive-definite weight matrices of proper dimension. $K_{c}$ is given by:

$$
K_{c}=R^{-1} B^{T} P
$$

where $P$ is the solution of the continuous time algebraic Riccati Equation given by

$$
A^{T} P+P A-P B R^{-1} P+Q=0
$$

It should be noted that full access to the states is assumed in this controller. In other words, it is assumed that all states of the system are measurable. In designing a controller, in addition to providing the stability of the system, it is also desirable that the outputs of the system converge to a desired set point value, like $r_{d}$. This can be achieved by making the state $x$ and the input $u$ of the process (3.22) converge 
to values $x^{*}$ and $u^{*}$ for which:

$$
\left\{\begin{array}{l}
0=A x^{*}+B u^{*} \\
r_{d}=C x^{*}
\end{array}\right.
$$

The first equation in (3.27) states that the output vector $y$ will converge to $r_{d}$ as $x$ and $u$ converge to $x^{*}$ and $u^{*}$ and the second equation implies that when these values are achieved, $\dot{x}=0$ and therefore $x$ will remain equal to $x^{*}$. In other words $x^{*}$ is an equilibrium state.

Given a desired set point $r_{d}$, it is possible to find $x^{*}$ and $u^{*}$ from the set of equations in (3.27) as given in (3.28). It is to be noted that unless there exists a zero at the origin of the transfer function between $y$ and $u$ (which will always make $y$ converge to zero when the input converges to a constant), the system of equations in (3.27) has at least one solution, that is

$$
\left\{\begin{array}{l}
x^{*}=F r_{d} \\
u^{*}=N r_{d}
\end{array}\right.
$$

where $F$ and $N$ could be found by solving the equation given in (3.27), that is

$$
\left[\begin{array}{ll}
A & B \\
0 & C
\end{array}\right]\left[\begin{array}{l}
x^{*} \\
u^{*}
\end{array}\right]=\left[\begin{array}{c}
0 \\
r_{d}
\end{array}\right] \Longrightarrow\left[\begin{array}{l}
x^{*} \\
u^{*}
\end{array}\right]=\left[\begin{array}{ll}
A & B \\
0 & C
\end{array}\right]^{-1}\left[\begin{array}{c}
0 \\
r_{d}
\end{array}\right]
$$

By setting the input signal $u$ as defined in (3.30) the output of the system $y$ will converge to the desired set point $r_{d}$, namely

$$
u=-K_{c}\left(x-x^{*}\right)+u^{*}
$$




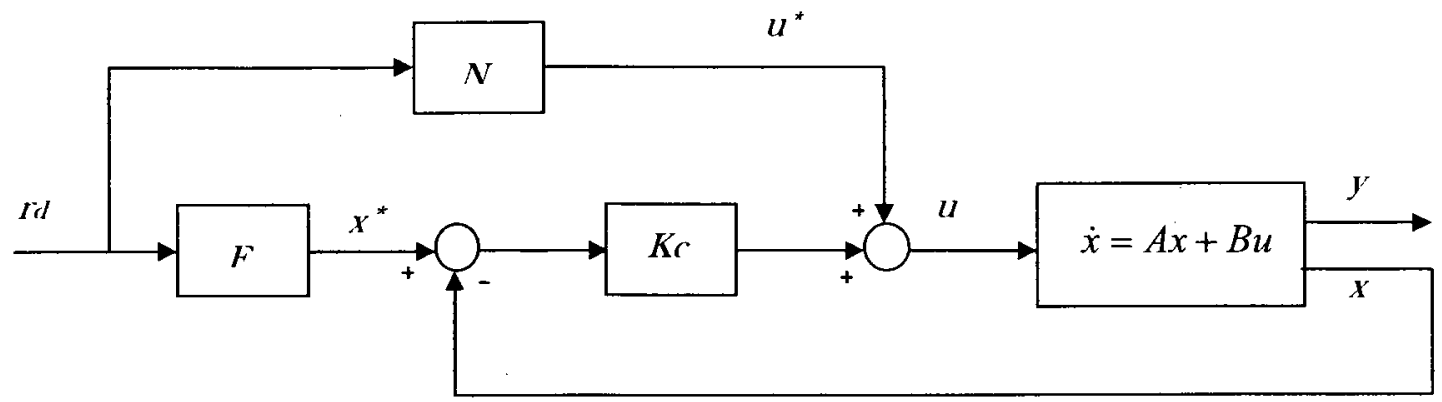

Figure 3.1: The schematic of the set-point optimal LQR control.

where $x^{*}$ and $u^{*}$ are given by (3.28) and $K_{c}$ is the optimum gain matrix. The state space model for the closed loop system is shown in Figure (3.3.1).

The closed loop system equations can be written as in (3.31).

$$
\left\{\begin{array}{l}
\dot{x}=A x+B u=\left(A-B K_{c}\right) x+B\left(K_{c} \dot{F}+N\right) r_{d} \\
y=C x
\end{array}\right.
$$

In order to show that by setting the input signal $u$ as stated in (3.30), the output of the system $y$ will converge to the desired set point $r_{d}$, suppose three new variables as defined in (3.32), namely

$$
\left\{\begin{array}{l}
\tilde{x}=x-x^{*} \\
\tilde{u}=u-u^{*} \\
\tilde{y}=y-r_{d}
\end{array}\right.
$$

where $x^{*}$ and $u^{*}$ are defined in (3.28). It is possible to show that:

$$
\left\{\begin{array}{l}
\dot{\tilde{x}}=A x+B u=A\left(x-x^{*}\right)+B\left(u-u^{*}\right)+A x^{*}+B u^{*} \\
\tilde{y}=C x-r_{d}=C\left(x-x^{*}\right)+C x^{*}-r_{d}
\end{array}\right.
$$


Considering the equation in (3.27), the above equations can be rewritten as

$$
\left\{\begin{array}{l}
\dot{\tilde{x}}=A \tilde{x}+B \tilde{u} \\
\tilde{y}=C \tilde{x}
\end{array}\right.
$$

The state feedback control law in (3.30) could also be represented as

$$
u-u^{*}=-K_{c}\left(x-x^{*}\right) \Longrightarrow \tilde{u}=-K_{c} \tilde{x}
$$

where $K_{c}$ is minimizing the following cost function:

$$
J_{0}=\int\left(\tilde{x}^{T} Q \tilde{x}+\tilde{u}^{T} R \tilde{u}\right) d t
$$

This controller makes the system (3.34) asymptotically stable, therefore $\tilde{x}, \tilde{u}$ and $\tilde{y}$, all converge to zero as $t \longrightarrow \infty$, which implies that $y$ converges to $r_{d}$.

\subsubsection{Feedback Linearization Control Design}

Feedback linearization (FL) is a common approach used in controlling nonlinear systems. The approach involves obtaining a transformation for the nonlinear system that maps the system into an equivalent linear system through a change of coordinates and a suitable control input.

Assume that the nonlinear system is defined by [64]:

$$
\left\{\begin{array}{l}
\dot{x}(t)=f(x(t))+g(x(t)) u(t) \\
y(t)=h(x(t))
\end{array}\right.
$$

where $u(t) \in \mathbb{R}, y(t) \in \mathbb{R}$ and $x(t) \in \mathbb{R}^{n}$ are the input, output and state vectors respectively. We assume that the vector fields $f: \mathrm{D} \longrightarrow \mathbb{R}^{\mathrm{n}}$ and $g: \mathrm{D} \longrightarrow \mathbb{R}^{\mathrm{n}}$ are 
smooth in the domain $\mathrm{D} \in \mathbb{R}^{\mathrm{n}}$, that is their partial derivatives with respect to $x$ of any order exist and are continuous in $\mathrm{D}$, and $h: \mathrm{D} \longrightarrow \mathbb{R}$ is also assumed to be a smooth scalar function. We are searching for a state feedback law that renders a linear input-output map between the new input $w$ and the output $y$. An outer-loop control strategy for the resulting linear control system can then be applied [65].

Definition 1. [65] Let $h: \mathrm{D} \longrightarrow \mathbb{R}$ be a smooth scalar function, and $f: \mathrm{D} \longrightarrow \mathbb{R}^{\mathrm{n}}$ be a smooth vector field on $\mathrm{D} \in \mathbb{R}^{\mathrm{n}}$, then the Lie derivative of $h$ with respect to $f$ is a scalar function defined by $L_{f} h=\nabla h f$.

In the above definition $\nabla h$ denotes to the gradient of $h$ as $\nabla h=\frac{\partial h}{\partial x}$. Thus, the Lie derivative $L_{f} h$ is simply the directional derivative of $h$ along the direction of the vector $f$.

In this work we have used input-output linearization [65] to find the control law. By input-output linearization we mean the generation of a linear differential relation between the output $y(t)$ and a new input $w$. The basic approach of inputoutput linearization is simply to differentiate the output function $y(t)$ repeatedly until the input $u(t)$ appears, and then design $u(t)$ to cancel the nonlinearities.

The process of repeated differentiation means that we start with:

$$
\dot{y}=\nabla h(x)(f(x)+g(x) u)=L_{f} h(x)+L_{g} h(x) u
$$

If $L_{g} h(x) \neq 0$ for some $x=x_{0}$, then the input transformation as defined in (3.39) will result in a linear relation between the output $y(t)$ and a the new input $w$ as given in (3.40), that is

$$
\begin{gathered}
u=\frac{1}{L_{g} h(x)}\left(-L_{f} h(x)+w\right) \\
\dot{y}=w
\end{gathered}
$$

If $L_{g} h(x)=0$ for all $x$ then the act of differentiation on $y(t)$ should be repeated 
for $i$ times as in (3.41), namely

$$
y^{(i)}=L_{f}^{i} h(x)+L_{g} L_{f}^{i-1} h(x) u
$$

until for an integer $i=r, L_{g} L_{f}^{r-1} h(x) \neq 0$ for some $x=x_{0}$. Then the control law given by $(3.42)$

$$
u=\frac{1}{L_{g} L_{f}^{i-1} h(x)}\left(-L_{f}^{r} h(x)+w\right)
$$

applied to the equation (3.41) while $i=r$, yields the following linear relation:

$$
y^{(r)}=w
$$

Definition 2. [17] The nonlinear system in (3.37) is said to have relative degree $r$, $1 \leq r \leq n$, in a region $\mathrm{D}_{0} \subset \mathrm{D}$ if

$$
L_{g} L_{f}^{i-1} h(x)=0, \quad i=1,2, \ldots, r-1 ; \quad L_{g} L_{f}^{r-1} h(x) \neq 0
$$

for all $x \in D_{0}$.

Definition 3. [17] A function $\Phi: \mathbb{R}^{n} \longrightarrow \mathbb{R}^{n}$, defined in a region $\mathrm{D}$ is called a diffeomorphism if it is smooth, and if its inverse $\Phi^{-1}$ exists and is smooth.

Lemma 1. [17] Let $\Phi(x)$ be a smooth function defined in a region $\mathrm{D}$ in $\mathbb{R}^{n}$. If the Jacobian matrix $\nabla \Phi$ is non-singular at a point $x=x_{0}$ of $\mathrm{D}$, then $\Phi(x)$ defines a local diffeomorphism in a subregion of $\mathrm{D}$ like $\mathrm{D}_{0}$.

Theorem 1. [65] Suppose that the nonlinear system in (3.37) has relative degree $r$ 
in $\mathrm{D}_{0}$. Let

$$
\begin{aligned}
\phi_{1}(x) & \doteq h(x) \\
\phi_{2}(x) & \doteq L_{f} h(x) \\
\vdots & \\
\phi_{r}(x) & \doteq L_{f}^{r-1} h(x)
\end{aligned}
$$

If $r<n$ there exists $(n-r)$ smooh functions $\phi_{r+1}(x), \ldots, \phi_{n}(x)$ such that the mapping

$$
\Phi(x)=\left[\begin{array}{c}
\phi_{1}(x) \\
\phi_{2}(x) \\
\vdots \\
\phi_{n}(x)
\end{array}\right]
$$

has a nonsingular Jacobian matrix in $\mathrm{D}_{0}$, and thus the $\Phi(x)$ defines a diffeomophism in a neighborhood of $x_{0} \in \mathrm{D}_{0}$. Furthermore, it is always possible to choose $\phi_{r+1}(x), \ldots, \phi_{n}(x)$ in such a way that

$$
L_{g} \phi_{i}(x)=0 \quad i=r+1, \ldots, n \quad \forall x \in \mathrm{D}_{0}
$$

The proof to this theorem can be found in [65], where it is shown that the gradients $\nabla \phi_{1}, \nabla \phi_{2}, \ldots, \nabla \phi_{n}$ are linearly independent, provided that $\phi_{r+1}(x), \ldots, \phi_{n}(x)$ are selected properly as stated in Theorem 1.

Therefore, it is possible to construct a coordinate transformation $Z=\Phi(x)$ that could map the nonlinear system (3.37) into a linear one [64]. The first $r$ 
components of $Z$ are exactly as

$$
\begin{aligned}
& z_{1}=y=h(x) \\
& z_{2}=\dot{y}=L_{f} h(x) \\
& \quad \vdots \\
& z_{r}=y^{(r-1)}=L_{f}^{r-1} h(x)
\end{aligned}
$$

The normal form [17] of the system can now be written as:

$$
\begin{aligned}
\dot{z}_{1} & =z_{2} \\
\dot{z}_{2} & =z_{3} \\
\vdots & \\
\dot{z}_{r-1} & =z_{r} \\
\dot{z}_{r} & =a(z)+b(z) u \\
\dot{z}_{r+1} & =q_{r+1}(z) \\
\vdots & \\
\dot{z}_{n} & =q_{n}(z) \\
y & =z_{1}
\end{aligned}
$$

where

$$
\left\{\begin{array}{l}
a(z)=\left.L_{f}^{r} h(x)\right|_{x=\Phi^{-1}(z)} \\
b(z)=\left.L_{g} L_{f}^{r-1} h(x)\right|_{x=\Phi^{-1}(z)}
\end{array}\right.
$$

and

$$
q_{i}(z)=\left.L_{f}^{i}\right|_{x=\Phi^{-1}(z)}
$$

By setting the input $u$ as in (3.42), the closed loop system in the normal form is 
obtained as

$$
\begin{gathered}
\dot{z}_{1}=z_{2} \\
\dot{z}_{2}=z_{3} \\
\vdots \\
\dot{z}_{r-1}=z_{r} \\
\dot{z}_{r}=w \\
\dot{z}_{r+1}=q_{r+1}(z) \\
\vdots \\
\dot{z}_{n}=q_{n}(z) \\
y=z_{1}
\end{gathered}
$$

It is seen that the control law has acted in such a way that the closed loop system has been partitioned into linear and nonlinear parts. More precisely, let $Z=$ $\left[\begin{array}{ll}\xi^{T} & \eta^{T}\end{array}\right]^{T}$ where $\xi=\left[\begin{array}{llll}z_{1} & z_{2} & \ldots & z_{r}\end{array}\right]^{T}$ and $\eta=\left[\begin{array}{llll}z_{r+1} & z_{r+2} & \ldots & z_{n}\end{array}\right]^{T}$. Therefore, the equations (3.49) could be rewritten as

$$
\left\{\begin{array}{l}
\dot{\xi}=A_{n} \xi+B_{n} w \\
\dot{\eta}=q(\xi, \eta) \\
y=C_{n} \xi
\end{array}\right.
$$

where $A_{n}, B_{n}$ and $C_{n}$ are the canonical representation form of the first $r$ equations in $(3.49)$ and $q(\xi, \eta)=\left[\begin{array}{lll}q_{r+1} & \ldots & q_{n}\end{array}\right]^{T}$.

Therefore, the dynamics of the nonlinear system is decomposed into an external (related to $\xi$ ) and an unobservable internal part (related to $\eta$ ). The external part consists of a linear relation between $y$ and $w$ and it is simply possible to design 
the input $w$ so that the output $y$ behaves as desired. Since the control design must account for the entire system dynamics, the internal behavior has to be studied to guarantee the stability of the entire system. The internal dynamics are described by:

$$
\dot{\eta}=q(\xi, \eta)
$$

Setting $\xi=0$ in (3.52) results in the following

$$
\dot{\eta}=q(0, \eta)
$$

which is called the zero dynamics [17].

The system is said to be minimum phase if equation (3.52) has an asymptotically stable equilibrium point [17]. In the special case where $r=n$, or in other words the relative degree of the system is equal to $n$, the normal form reduces to

$$
\left\{\begin{array}{l}
\dot{\xi}=A_{n} \xi+B_{n} w \\
y=C_{n} \xi
\end{array}\right.
$$

where $Z=\xi=\left[\begin{array}{llll}z_{1} & z_{2} & \ldots & z_{n}\end{array}\right]^{T}$ and the $\eta$ variable does not exist. In this case the system has no zero dynamics and by default is said to be minimum phase [17].

\subsubsection{Quadrotor Control Scheme Design}

At the end of section (3.2) the entire dynamic equations describing the quadrotor system were derived in (3.21). It should be noted that the dynamic equation related to the yaw $\psi$ is controlled by $U_{4}$ and $v_{4}$. Therefore, it is possible to design a controller 
for this decoupled subsystem $\left(\psi-U_{4}\right)$, namely

$$
\left\{\begin{array}{l}
\ddot{\psi}=\frac{U_{4}}{I_{z z}} \\
\dot{U}_{4}=A_{t} U_{4}+B_{t} v_{4}
\end{array}\right.
$$

For the above linear subsystem an LQR controller is designed by considering the following state space representation,

$$
\begin{gathered}
X=\left[\begin{array}{l}
x_{1} \\
x_{2} \\
x_{3}
\end{array}\right]=\left[\begin{array}{c}
\psi \\
\dot{\psi} \\
U_{4}
\end{array}\right] \\
\dot{X}=\left[\begin{array}{lll}
0 & 1 & 0 \\
0 & 0 & \frac{1}{I_{z z}} \\
0 & 0 & A_{t}
\end{array}\right]+\left[\begin{array}{c}
0 \\
0 \\
B_{t}
\end{array}\right] v_{4} \\
y=\left[\begin{array}{lll}
1 & 0 & 0
\end{array}\right]^{T} X
\end{gathered}
$$

By setting $Q=I_{(3 \times 3)}$ and $R=1$ and solving the Riccati Equation in (3.26), the gain vector $K_{\psi}=\left[\begin{array}{lll}k_{\psi 1} & k_{\psi 2} & k_{\psi 3}\end{array}\right]^{T}$ is obtained. By setting the control input $v_{4}$ as in (3.57), the $\left(\psi-U_{4}\right)$ subsystem becomes asymptotically stable as stated in Section 3.3 .1 , namely

$$
v_{4}=-K_{\psi}\left[\begin{array}{c}
\psi \\
\dot{\psi} \\
U_{4}
\end{array}\right]
$$

It should be noted that in this control law the desired output is set to zero, that is $\psi^{*} \longrightarrow 0$. 
Now, the remaining variables to be controlled are $x, y$ and $z$. It is possible to assume that the altitude $z$ could be controlled by $U_{1}$. The dynamics governing the $z$ is given by

$$
\left\{\begin{array}{l}
\ddot{z}=-g+(\cos \phi \cos \theta) \frac{U_{1}}{m} \\
\dot{U}_{1}=A_{t} U_{1}+B_{t} v_{1}+4 C_{t}
\end{array}\right.
$$

If the altitude $z$ reaches a desired set-point like $z_{d}$, then $\ddot{z} \longrightarrow 0$. As stated before, in hovering condition the total thrust should be equal to the gravitational force effective on the quadrotor, in other words:

$$
U_{1}=\sum_{i=1}^{4} T_{i}=m g
$$

Therefore, if $\ddot{z} \longrightarrow 0$ and $\phi$ and $\theta$ are sufficiently close to zero, then $U_{1} \longrightarrow m g$.

By assuming $U_{1} \longrightarrow m g$ and $\psi \longrightarrow 0$, it is possible to simplify the dynamic equations of $x$ and $y$ defined by (3.21) as given by equations (3.60) and (3.61), that is

$$
\begin{aligned}
& \left\{\begin{array}{l}
\ddot{y}=-g \sin \phi \\
\ddot{\phi}=\frac{U_{2}}{I_{x x}} \\
\dot{U}_{2}=A_{t} U_{2}+B_{t} v_{2}
\end{array}\right. \\
& \left\{\begin{array}{l}
\ddot{x}=g \sin \theta \\
\ddot{\theta}=\frac{U_{3}}{I_{y y}} \\
\dot{U_{3}}=A_{t} U_{3}+B_{t} v_{3}
\end{array}\right.
\end{aligned}
$$

Through these assumptions, which would be verified by simulations, the dynamics of the quadrotor in (3.21) is partitioned into four semi-decoupled subsystems of $\left(z, U_{1}\right),\left(y, U_{2}\right),\left(x, U_{3}\right)$ and $\left(\psi, U_{4}\right)$ as defined in $(3.58),(3.60),(3.61)$ and $(3.54)$ respectively. The reason for calling these subsystems semi-decoupled is because $z$ is 
still coupled with $\phi$ and $\theta$.

For the control of the altitude $z$, the input-output linearization technique described in section (3.3.2) is employed to derive a linear relation between the output and a new input variable.

By differentiating the dynamic equation of $z$ as defined in (3.58) once, we have

$$
z^{(3)}=-\dot{\phi} \sin \phi \cos \theta \frac{U_{1}}{m}-\dot{\theta} \cos \phi \sin \theta \frac{U_{1}}{m}+\cos \phi \cos \theta \frac{\dot{U}_{1}}{m}
$$

By substituting the equation for $\dot{U}_{1}$ from (3.58) in the above equation, the input $v_{1}$ appears as in (3.63), that is

$$
z^{(3)}=-\dot{\phi} \sin \phi \cos \theta \frac{U_{1}}{m}-\dot{\theta} \cos \phi \sin \theta \frac{U_{1}}{m}+\cos \phi \cos \theta \frac{A_{t} U_{1}+B_{t} v_{1}+4 C_{t}}{m}
$$

Therefore, the relative degree of the nonlinear system in (3.58) is equal to $n=3$. The normal coordinates are defined according to

$$
\Phi_{z}=\left[\begin{array}{c}
z \\
\dot{z} \\
\ddot{z}
\end{array}\right]
$$

which is a diffeomorphism on the region $\mathbb{R}^{3}$. There is no internal dynamics for this nonlinear system and it is minimum phase.

By setting $v_{1}$ as in (3.65), a linear relation between $z^{(3)}$ and the new input $w_{1}$ is obtained, namely

$$
v_{1}=\frac{1}{\frac{B_{t}}{m} \cos \phi \cos \theta}\left(w_{1}+\dot{\phi} \sin \phi \cos \theta \frac{U_{1}}{m}+\dot{\theta} \cos \phi \sin \theta \frac{U_{1}}{m}-\cos \phi \cos \theta \frac{A_{t} U_{1}+4 C_{t}}{m}\right)
$$

Therefore,

$$
z^{(3)}=w_{1}
$$


It should be noted that the input $v_{1}$ is just valid for the case that $\cos \phi \cos \theta \neq 0$.Local asymptotic stability is achieved by setting the new input $w_{1}$ as:

$$
w_{1}=z_{d}^{(3)}-k_{1 z}\left(\ddot{e}_{z}\right)-k_{2 z}\left(\dot{e}_{z}\right)-k_{3 z}\left(e_{z}\right)
$$

where $z_{d}$ refers to the desired altitude set point, $e_{z}$ refers to the error $e_{z}=z-z_{d}$ and the gains $K_{z}=\left[\begin{array}{lll}k_{1 z} & k_{2 z} & k_{3 z}\end{array}\right]^{T}$ are optimally obtained from the LQR method as described in section (3.3.1).

The error dynamic equation is now derived by substituting (3.67) into (3.66), that is

$$
e_{z}^{(3)}+k_{1 z}\left(\ddot{e}_{z}\right)+k_{2 z}\left(\dot{e}_{z}\right)+k_{3 z}\left(e_{z}\right)=0
$$

which yields an asymptotically stable error dynamics that converges to zero as $t \longrightarrow$ $\infty$. Therefore, the subsystem $\left(z, U_{1}\right)$ is stable, that is $z \longrightarrow z_{d}$ and $U_{1} \longrightarrow m g$.

The same procedure can be followed to obtain the control laws for $\left(y, U_{2}\right)$ and $\left(x, U_{3}\right)$ subsystems as defined in equations (3.60) and (3.61). To obtain the input controller, we differentiate repeatedly from the $\ddot{y}$ and $\ddot{x}$ until the input terms $v_{2}$ and $v_{3}$ appear, that is

$$
\begin{gathered}
\left\{\begin{array}{l}
y^{(3)}=-g \dot{\phi} \cos \phi \\
x^{(3)}=g \dot{\theta} \cos \theta
\end{array}\right. \\
\left\{\begin{array}{l}
y^{(4)}=-g \ddot{\phi} \cos \phi+g \dot{\phi}^{2} \sin \phi \\
x^{(4)}=g \ddot{\theta} \cos \theta-g \dot{\theta}^{2} \sin \theta
\end{array}\right.
\end{gathered}
$$

Substituting $\ddot{\phi}$ and $\ddot{\theta}$ from (3.60) and (3.61) results in:

$$
\left\{\begin{array}{l}
y^{(4)}=-g \frac{U_{2}}{I_{x x}} \cos \phi+g \dot{\phi}^{2} \sin \phi \\
x^{(4)}=g \frac{U_{3}}{I_{y y}} \cos \theta-g \dot{\theta}^{2} \sin \theta
\end{array}\right.
$$


By another differentiation the inputs $v_{2}$ and $v_{3}$ would appear in the equations, namely

$$
\begin{gathered}
\left\{\begin{array}{l}
y^{(5)}=-g \frac{\dot{U}_{2}}{I_{x x}} \cos \phi+g \frac{U_{2}}{I_{x x}} \dot{\phi} \sin \phi+2 g \dot{\phi} \ddot{\phi} \sin \phi+g \dot{\phi}^{3} \cos \phi \\
x^{(5)}=g \frac{\dot{U}_{3}}{I_{y y}} \cos \theta-g \frac{U_{3}}{I_{y y}} \dot{\theta} \sin \theta-2 g \dot{\theta} \ddot{\theta} \sin \theta-g \dot{\theta}^{3} \cos \theta
\end{array}\right. \\
\left\{\begin{array}{l}
y^{(5)}=-g \frac{A_{t} U_{2}+B_{t} v_{2}}{I_{x x}} \cos \phi+g \frac{U_{2}}{I_{x x}} \dot{\phi} \sin \phi+2 g \dot{\phi} \frac{U_{2}}{I_{x x}} \sin \phi+g \dot{\phi}^{3} \cos \phi \\
x^{(5)}=g \frac{A_{t} U_{3}+B_{t} v_{3}}{I_{y y}} \cos \theta-g \frac{U_{3}}{I_{y y}} \dot{\theta} \sin \theta-2 g \dot{\theta} \frac{U_{3}}{I_{y y}} \sin \theta-g \dot{\theta}^{3} \cos \theta
\end{array}\right.
\end{gathered}
$$

where (3.73) is obtained by replacing $\dot{U}_{2}$ and $\dot{U}_{3}$ by (3.60) and (3.61) in (3.72). It is seen that the relative degrees for these two nonlinear subsystems is equal to $r=n=5$. The normal coordinates are defined in (3.74) and (3.75), that is

$$
\begin{gathered}
\Phi_{y}=\left[\begin{array}{c}
y \\
\dot{y} \\
\ddot{y} \\
y^{(3)} \\
y^{(4)}
\end{array}\right] \\
\Phi_{x}=\left[\begin{array}{c}
x \\
\dot{x} \\
\ddot{x} \\
x^{(3)} \\
x^{(4)}
\end{array}\right]
\end{gathered}
$$

which represents diffeomorphisms on $\mathbb{R}^{5}$. Moreover, since the relative degrees are equal to the systems order, there are no internal dynamics for these two subsystems to be analyzed for stability. 
By setting the control variables $v_{2}$ and $v_{3}$ from (3.76), namely

$$
\left\{\begin{array}{l}
v_{2}=\frac{1}{-g \frac{B_{t}}{I_{x x}} \cos \phi}\left(w_{2}+g \frac{A_{t} U_{2}}{I_{x x}} \cos \phi-g \frac{U_{2}}{I_{x x}} \dot{\phi} \sin \phi-2 g \dot{\phi} \frac{U_{2}}{I_{x x}} \sin \phi-g \dot{\phi}^{3} \cos \phi\right) \\
v_{3}=\frac{1}{g \frac{B_{t}}{I_{y y}} \cos \theta}\left(w_{3}-g \frac{A_{t} U_{3}}{I_{y y}} \cos \theta+g \frac{U_{3}}{I_{y y}} \dot{\theta} \sin \theta+2 g \dot{\theta} \frac{U_{3}}{I_{y y}} \sin \theta+g \dot{\theta}^{3} \cos \theta\right)
\end{array}\right.
$$

a linear relation between $y^{(5)}$ and $x^{(5)}$ and the new inputs $w_{2}$ and $w_{3}$ is obtained as

$$
\left\{\begin{array}{l}
y^{(5)}=w_{2} \\
x^{(5)}=w_{3}
\end{array}\right.
$$

It should be noted that $v_{2}$ and $v_{3}$ are only valid if $\cos \phi \neq 0$ and $\cos \theta \neq 0$.

The new inputs $w_{2}$ and $w_{3}$ are selected as :

$$
\left\{\begin{array}{l}
w_{2}=y_{d}^{(5)}-k_{1 y} e_{y}^{(4)}-k_{2 y} e_{y}^{(3)}-k_{3 y} \ddot{e}_{y}-k_{4 y} \dot{e}_{y}-k_{5 y} e_{y} \\
w_{3}=x_{d}^{(5)}-k_{1 x} e_{x}^{(4)}-k_{2 x} e_{x}^{(3)}-k_{3 x} \ddot{e}_{x}-k_{4 x} \dot{e}_{x}-k_{5 x} e_{x}
\end{array}\right.
$$

where $y_{d}$ and $x_{d}$ refer to the desired set points for $y$ and $x, e_{y}=y-y_{d}$ and $e_{x}=x-x_{d}$ are defined as the error signals. The gain vectors $K_{y}=\left[\begin{array}{lllll}k_{1 y} & k_{2 y} & k_{3 y} & k_{4 y} & k_{5 y}\end{array}\right]^{T}$ and $K_{x}=\left[\begin{array}{lllll}k_{1 x} & k_{2 x} & k_{3 x} & k_{4 x} & k_{5 x}\end{array}\right]^{T}$ are obtained from the LQR method that is described in section (3.3.1).

The error dynamics are then obtained from (3.77) and (3.78) as :

$$
\left\{\begin{array}{l}
e_{y}^{(5)}+k_{1 y} e_{y}^{(4)}+k_{2 y} e_{y}^{(3)}+k_{3 y} \ddot{e}_{y}+k_{4 y} \dot{e}_{y}+k_{5 y} e_{y}=0 \\
e_{x}^{(5)}+k_{1 x} e_{x}^{(4)}+k_{2 x} e_{x}^{(3)}+k_{3 x} \ddot{e}_{x}+k_{4 x} \dot{e}_{x}+k_{5 x} e_{x}=0
\end{array}\right.
$$

Therefore asymptotically stable error dynamics are obtained from the above selected control inputs, where $e_{y}$ and $e_{x}$ converge to zero as $t \longrightarrow \infty$. In other words, $y \longrightarrow y_{d}$ and $x \longrightarrow x_{d}$ as $t \longrightarrow \infty$. 


\subsection{Simulation Results}

In this section the behavior of the quadrotor system with the designed FL controller and under normal conditions is evaluated through numerical simulations by commanding the control system to follow a desired trajectory. The parameters of the model are taken from the model OS4 developed in Ecole Polythechnique Federal De Lausanne [1]. The physical parameters of the OS4 quadrotor are shown in Table 3.1. Table 3.2 also shows the parameters that are related to the propulsion group consisting of the DC motor, gearbox and the propellers. All the simulations are done for the nonlinear model of the quadrotor and actuators as explained in the previous chapter by equations (2.39), (2.38) and (2.52).

Table 3.1: OS4 Quadrotor physical parameters [1]

\begin{tabular}{cccc}
\hline Name & Parameter & Value & Unit \\
\hline mass & $m$ & 0.650 & $\mathrm{~kg}$ \\
inertia on $x$ axis & $I_{x x}$ & $7.5 \mathrm{e}-3$ & $\mathrm{~kg} \cdot \mathrm{m}^{2}$ \\
inertia on $y$ axis & $I_{y y}$ & $7.5 \mathrm{e}-3$ & $\mathrm{~kg} \cdot \mathrm{m}^{2}$ \\
inertia on $z$ axis & $I_{z z}$ & $1.3 \mathrm{e}-2$ & $\mathrm{~kg} \cdot \mathrm{m}^{2}$ \\
thrust coefficient & $b$ & $3.13 \mathrm{e}-5$ & $\mathrm{Ns}{ }^{2}$ \\
drag coefficient & $d$ & $7.5 \mathrm{e}-7$ & $\mathrm{Nms}$ \\
arm length & $l$ & 0.23 & $\mathrm{~m}$ \\
& & & \\
\hline
\end{tabular}


Table 3.2: OS4 Quadrotor physical parameters [1]

\begin{tabular}{cccc}
\hline \hline Name & Parameter & Value & Unit \\
\hline propeller inertia & $J_{p}$ & $6 \mathrm{e}-5$ & $\mathrm{~kg} \cdot \mathrm{m}^{2}$ \\
gearbox efficiency & $\eta$ & 90 & $\%$ \\
gearbox ratio & $r$ & $4: 1$ & \\
motor inertia & $J_{m}$ & $4 \mathrm{e}-7$ & $\mathrm{~kg} \cdot \mathrm{m}^{2}$ \\
motor internal resistance & $R_{\text {mot }}$ & 0.6 & $\Omega$ \\
back-EMF constant & $k_{e}$ & 5.2 & $\mathrm{~V} \mathrm{~s} \mathrm{rad}$ \\
& & & \\
\hline
\end{tabular}

\subsubsection{Healthy System Response to 1st Trajectory}

In this section the response of the healthy system to a simple trajectory is presented. The mission starts at the position $(x, y, z)=(0,0,0)$ while the roll, the pitch and the yaw angles are initially equal to zero. The commanded trajectory is to fly from the initial point to the point $(10,10,10)(m)$ in 20 seconds and hovering at this point.

In order to simulate a more realistic environment, we added noise to the output signals as well as input signals. The noise is chosen to be an additive white Gaussian noise and the power of the noise is set to a value so that the signal to noise ratio (SNR) is a prespecified value, which represents a practical cnvironment [1]. The SNRs are as follows:

$$
\begin{aligned}
& S N R_{x}=15.59 d b \\
& S N R_{y}=15.35 d b
\end{aligned}
$$




$$
S N R_{z}=15.85 \mathrm{db}
$$

Figures 3.2 and 3.3 show the linear position and the Euler angles of the system with respect to time as a response to the commanded trajectory. It is seen that the response of the system to this trajectory is acceptable in the sense of meeting design requirements. The error signals between the desired commanded trajectory and the observed ones are depicted in Figure 3.6. Table 3.3 shows the mean and standard deviation of the error signals for $x, y, z$ and $\psi$ angle.

Table 3.3: Mean and standard deviation (Stdv) of the error signals for the $1^{\text {st }}$ trajectory

\begin{tabular}{cccc}
\hline \hline Error & Mean & Stdv & Unit \\
\hline$e_{x}$ & -0.0760 & 0.2938 & $\mathrm{~m}$ \\
$e_{y}$ & -0.1393 & 0.4008 & $\mathrm{~m}$ \\
$e_{z}$ & -0.0577 & 0.1869 & $\mathrm{~m}$ \\
$e_{\psi}$ & $1.6597 \mathrm{e}-004$ & 0.0011 & $\mathrm{rad}$ \\
\hline
\end{tabular}

It should be noted that the error signals are bounded within less than $1.5 \%$ of the steady state value which satisfies the design criteria according to [1] which is $2 \%$.

Figures 3.4 and 3.5 show the input voltage signal to the rotors and the movement moments. As expected, $U_{1} \longrightarrow m g$ as the system altitude has been stabilized and at hovering condition.

In order to check to robustness of the designed nominal controller to parameter variations, we have performed the simulations while the parameters of the body inertia matrix $I_{x x}, I_{y y}$ and $I_{z z}$ are changed by $10 \%$. The resulting error signals are depicted in Figure 3.7. Table 3.4 shows the mean and standard deviation of the error signals for $x, y, z$ and $\psi$ angle while there are parameter variations in the model of the system. 
Table 3.4: Mean and standard deviation (Stdv) of the error signals for the $1^{\text {st }}$ trajectory with $10 \%$ variations in the body inertia parameters

\begin{tabular}{cccc}
\hline \hline Error & Mean & Stdv & Unit \\
\hline$e_{x}$ & -0.0787 & 0.2921 & $\mathrm{~m}$ \\
$e_{y}$ & -0.1400 & 0.4012 & $\mathrm{~m}$ \\
$e_{z}$ & -0.0578 & 0.1865 & $\mathrm{~m}$ \\
$e_{\psi}$ & $-4.5996 \mathrm{e}-005$ & $8.1810 \mathrm{e}-004$ & $\mathrm{rad}$ \\
\hline
\end{tabular}

It is seen that the controller is totally robust to $10 \%$ variations in the parameters of the body inertia matrix and neglectable diffrences between the results from Table 3.4 to the results from Table 3.3 obtained with no parameter variations.

\subsubsection{Healthy System Response to 2nd Trajectory}

The second set of simulation results is related to the trajectory that is defined as in Table 3.6 while the quadrotor is commanded to move and reach certain points in space and hover for a specified amount of time. In this trajectory, the initial state of the quadrotor is assumed to be at the $(0,0,0)$ position and the roll, the pitch and the yaw angles are also assumed to be zero at the initial time.

Figures 3.8 and 3.10 show the linear position and the Euler angles of the system with respect to time as a response to the commanded trajectory shown in Figure 3.5. It is seen that the response of the system to this trajectory is acceptable. The error signals between the desired commanded trajectory and the observed ones are depicted in Figure 3.13. Table 3.5 shows the mean and standard deviation of the error signals for $x, y, z$ and $\psi$ angle. It is seen that the error signals are still bounded within an acceptable range of less than $1.5 \%$.

Figures 3.11 and 3.12 show the input voltage signal to the rotors and the 
Table 3.5: Mean and standard deviation (Stdv) of the error signals for the $2^{\text {nd }}$ trajectory.

\begin{tabular}{cccc}
\hline \hline Error & Mean & Stdv & Unit \\
\hline$e_{x}$ & 0.0105 & 0.5777 & $\mathrm{~m}$ \\
$e_{y}$ & -0.0110 & 0.3872 & $\mathrm{~m}$ \\
$e_{z}$ & -0.0013 & 0.3960 & $\mathrm{~m}$ \\
$e_{\psi}$ & $5.3510 \mathrm{e}-005$ & $7.1473 \mathrm{e}-004$ & $\mathrm{rad}$ \\
\hline
\end{tabular}

movement moments. As expected, $U_{1} \longrightarrow m g$ as the system altitude has been stabilized at hovering condition.

\subsection{Conclusions}

In this chapter the dynamics of the quadrotor model are partitioned into four semidecoupled subsystems by considering some approximations at the hovering condition. Furthermore, the dynamics governing the input moments to the quadrotor are derived and considered in the controller design for the system. By using the FL and the LQR methods, controllers are designed for the nonlinear subsystems and analysis is performed to show that the closed-loop system is asymptotically stable. Simulation results are also presented to show the performance of the nominal controller under different trajectories in healthy condition.

The next chapter will discuss the effects of different actuator faults on the quadrotor performance and a fault recovery module is proposed to compensate the effect of these faults on the system. 
Table 3.6: Second Trajectory

\begin{tabular}{|c|c|c|c|}
\hline$\overline{\text { Time(sec) }}$ & Position $(x, y, z)(\mathrm{m})$ & State & Duration \\
\hline 0 & $(0,0,0)$ & vertical flight to next position & $5 \mathrm{sec}$ \\
\hline 5 & $(0,0,10)$ & hover & $55 \mathrm{sec}$ \\
\hline 60 & $(0,0,10)$ & horizontal flight to next position & $5 \mathrm{sec}$ \\
\hline 65 & $(10,0,10)$ & hover & $55 \mathrm{sec}$ \\
\hline 120 & $(10,0,10)$ & horizontal flight to next position & $20 \mathrm{sec}$ \\
\hline 140 & $(10,10,10)$ & hover & $40 \mathrm{sec}$ \\
\hline 180 & $(10,10,10)$ & vertical flight to next position & $5 \mathrm{sec}$ \\
\hline 185 & $(10,10,0)$ & hover & $55 \mathrm{sec}$ \\
\hline 240 & $(10,10,0)$ & horizontal flight to next position & $5 \mathrm{sec}$ \\
\hline 245 & $(0,10,0)$ & hover & $55 \mathrm{sec}$ \\
\hline 300 & $(0,10,0)$ & horizontal flight to next position & $20 \mathrm{sec}$ \\
\hline 320 & $(0,0,0)$ & back to the starting point & ended \\
\hline
\end{tabular}


(a)

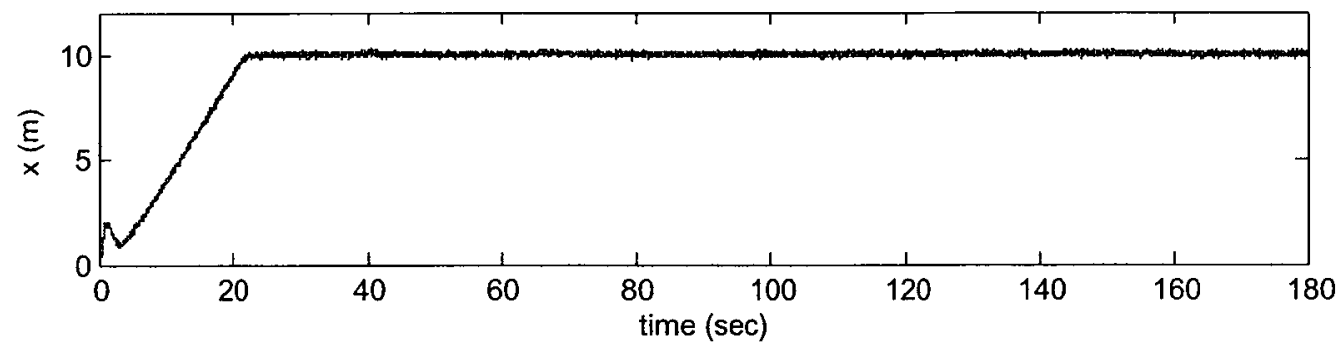

(b)

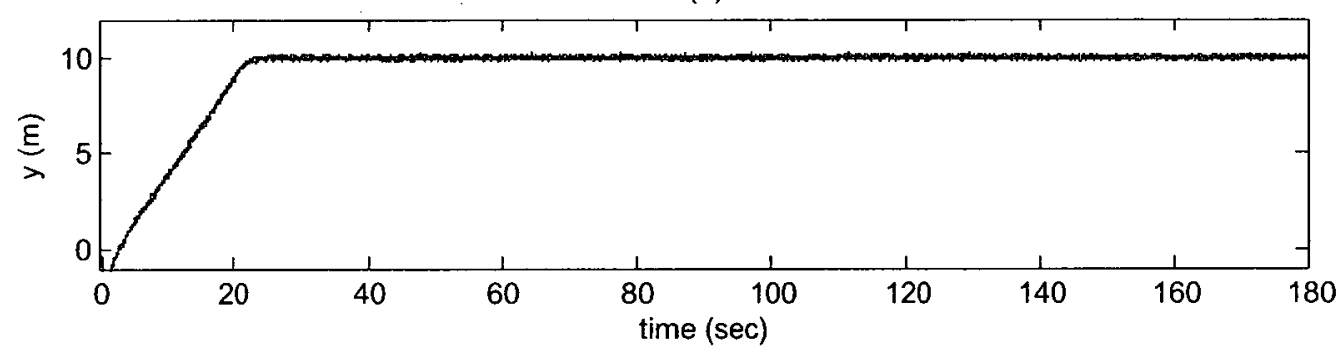

(c)

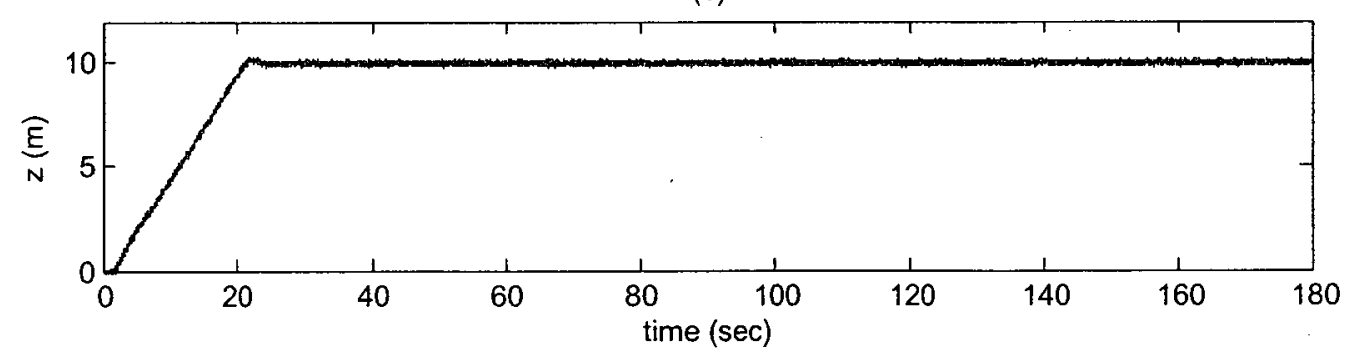

Figure 3.2: Linear position response to the commanded 1st trajectory : (a) $x$, (b) $y$ and (c) $z$ measured in meters. 
(a)

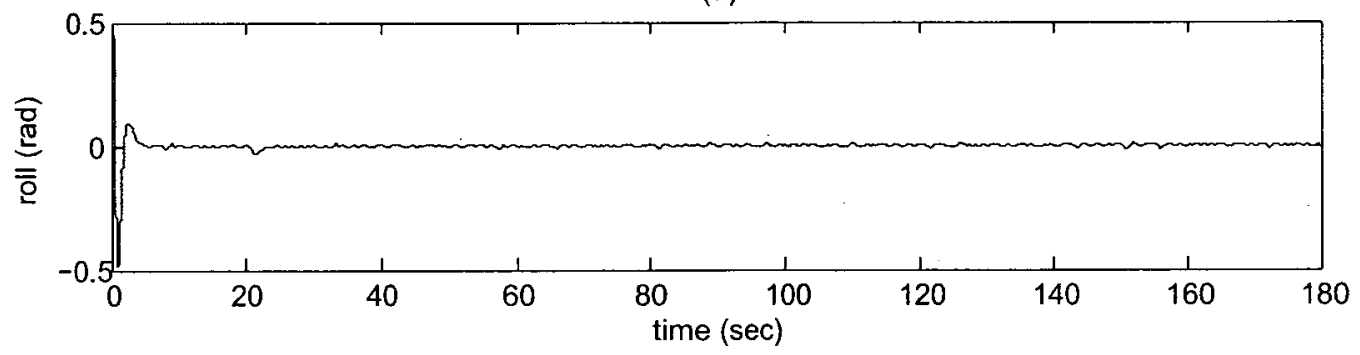

(b)

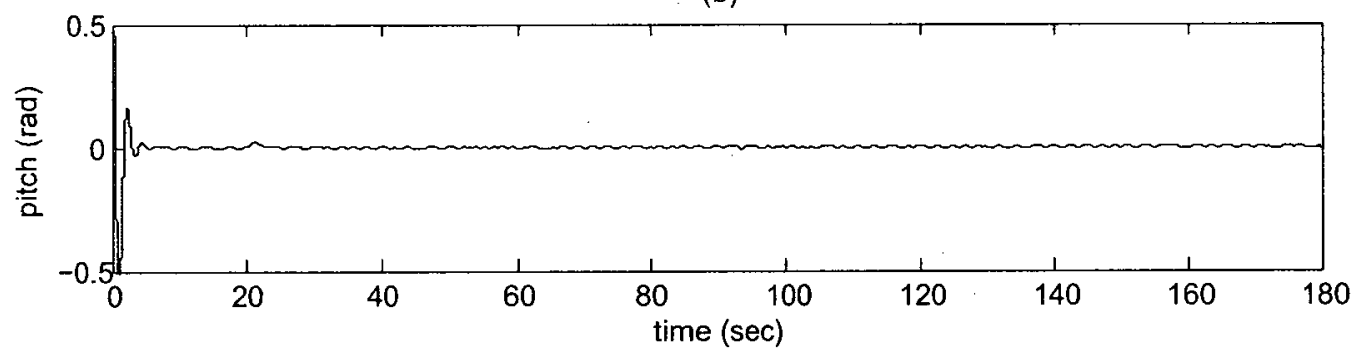

(c)

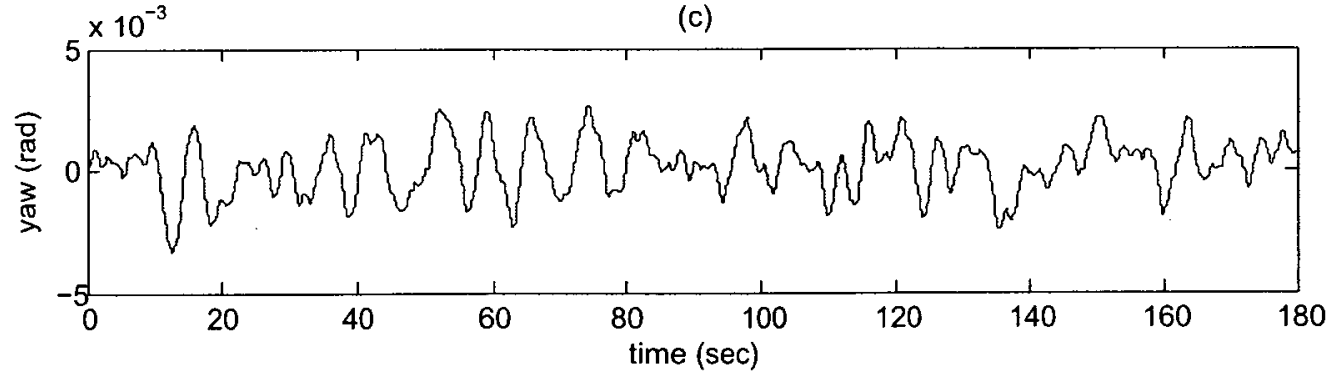

Figure 3.3: Euler angles in response to the commanded 1st trajectory : (a) Roll (rad), (b) Pitch (rad) and (c) Yaw (rad). 
(a)

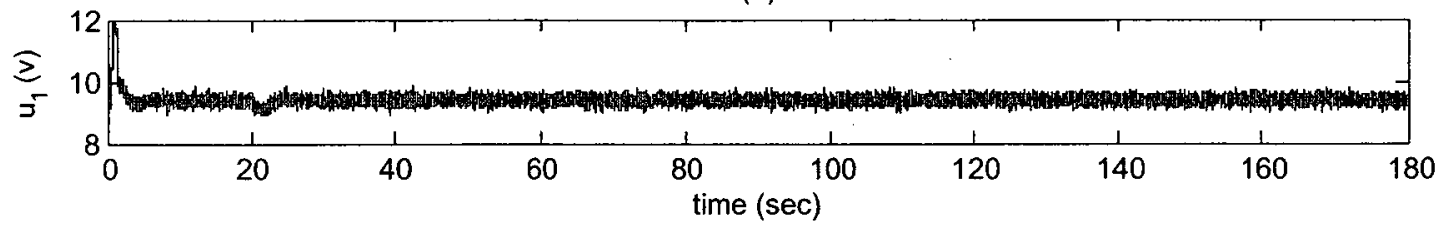

(b)

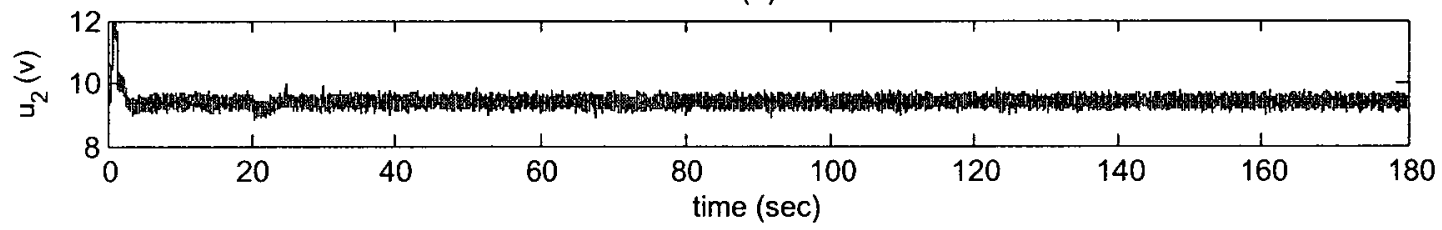

(c)

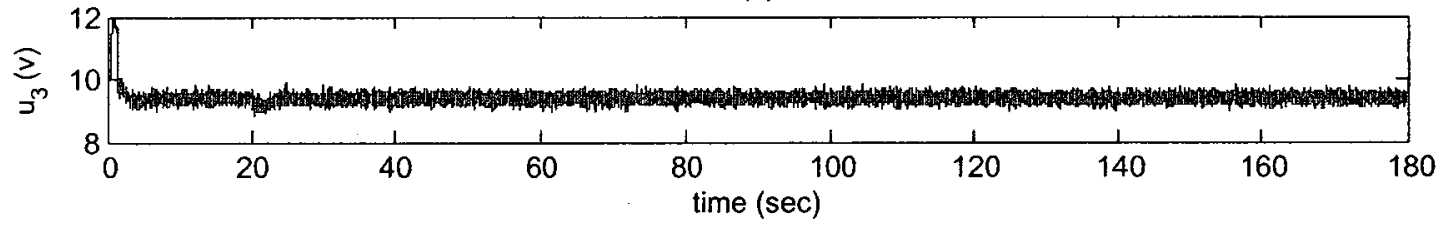

(d)

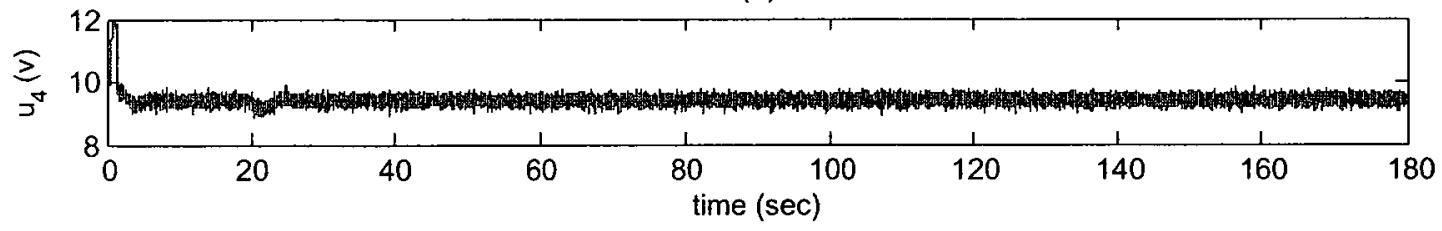

Figure 3.4: Input voltage to the 4 propellers in response to the commanded 1st trajectory. 
(a)

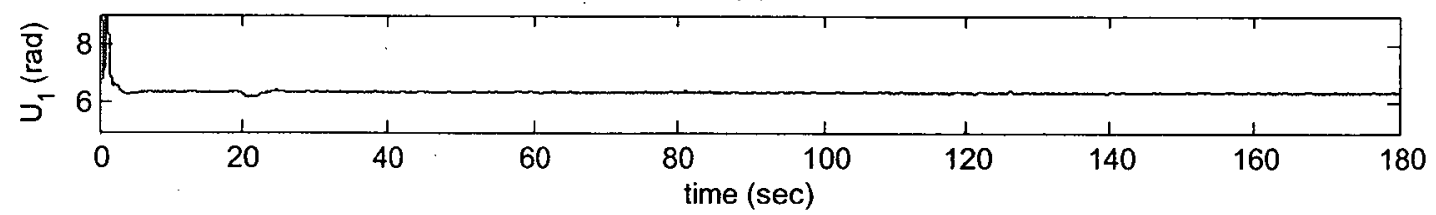

(b)

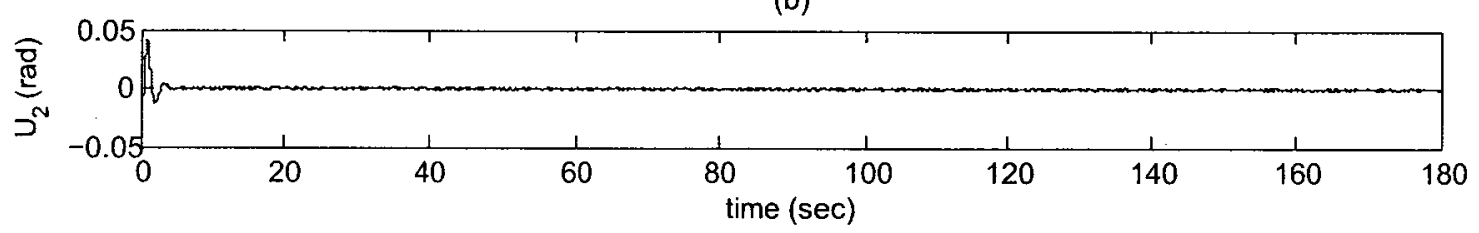

(c)

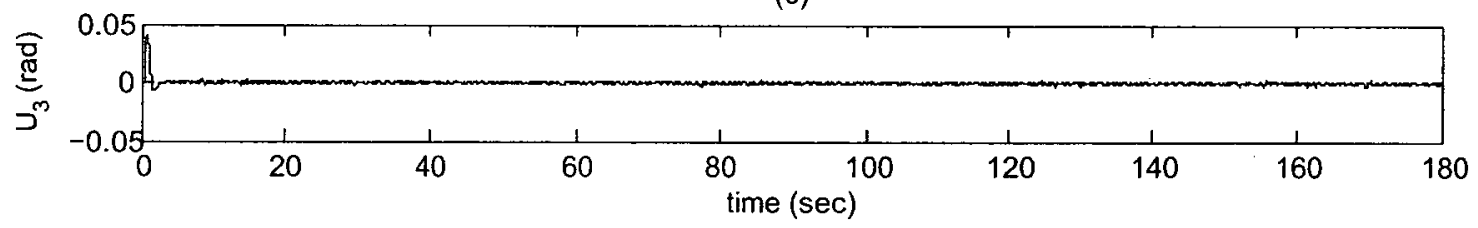

(d)

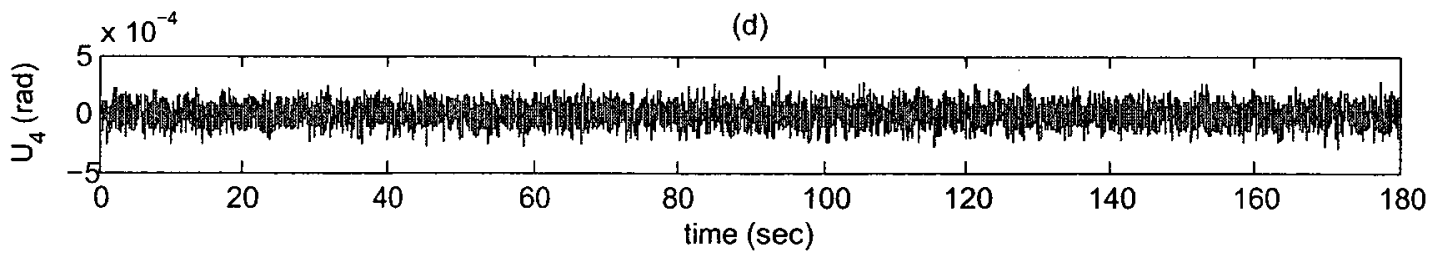

Figure 3.5: Input moments $U_{1}, U_{2}, U_{3}$ and $U_{4}$ to the dynamics of the quadrotor system in response to the commanded 1st trajectory. 
(a)

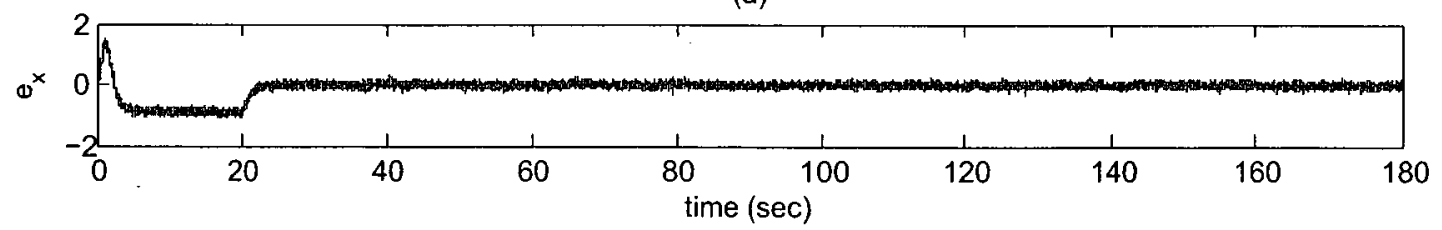

(b)

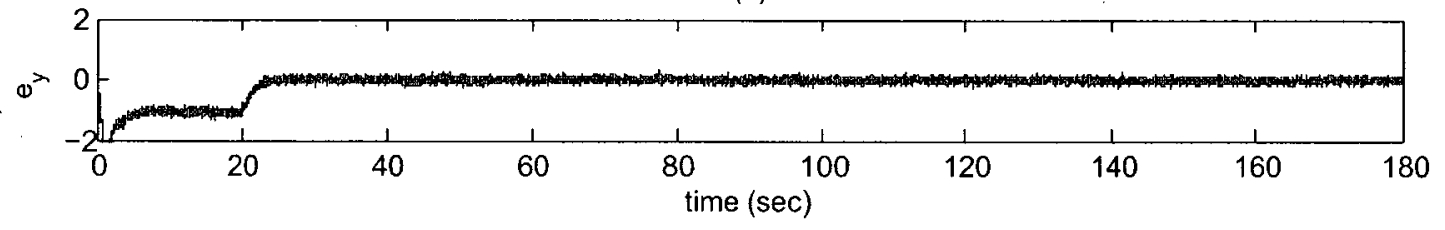

(c)
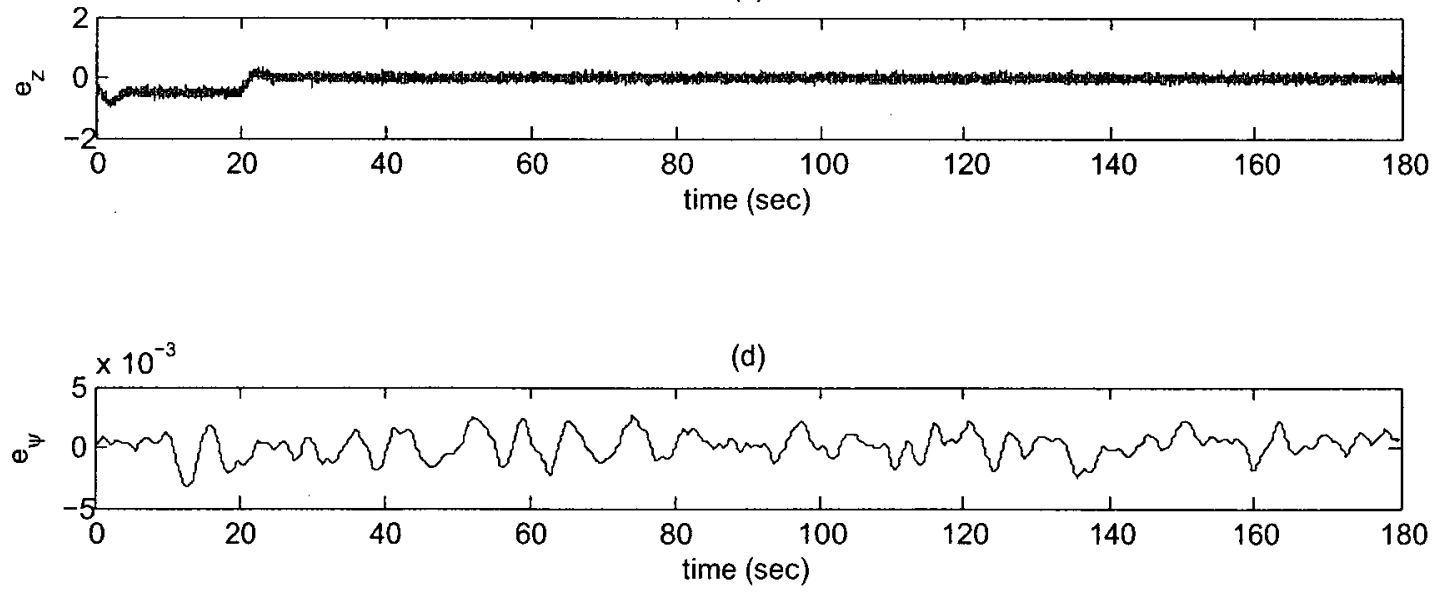

Figure 3.6: Error signals $e_{x}, e_{y}, e_{z}$ measured in meters and $e_{\psi}$ in radian in response to the commanded 1st trajectory. 
(a)

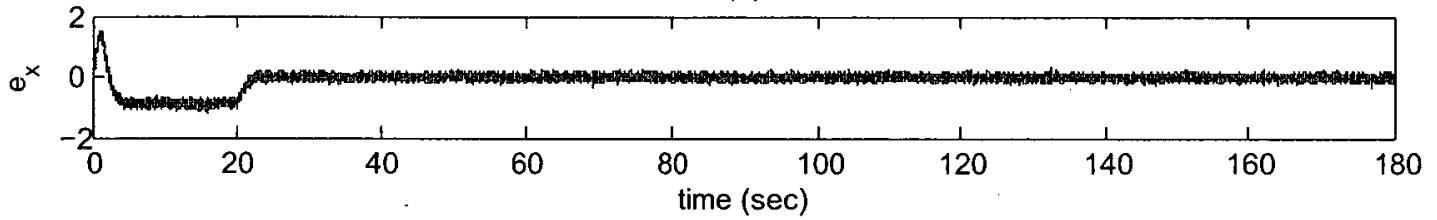

(b)

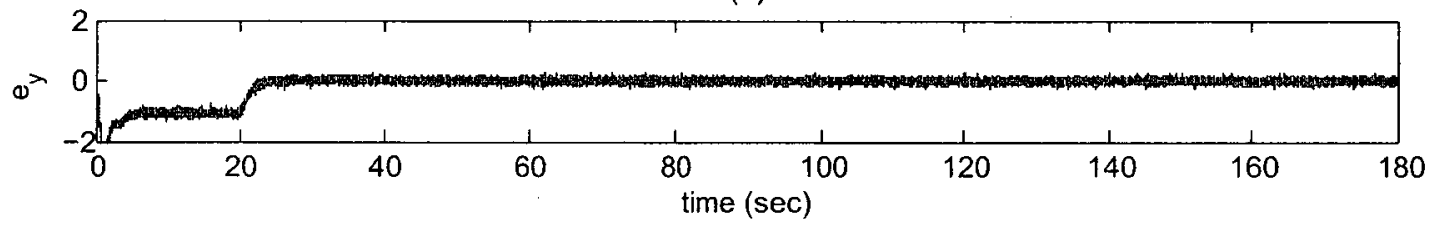

(c)
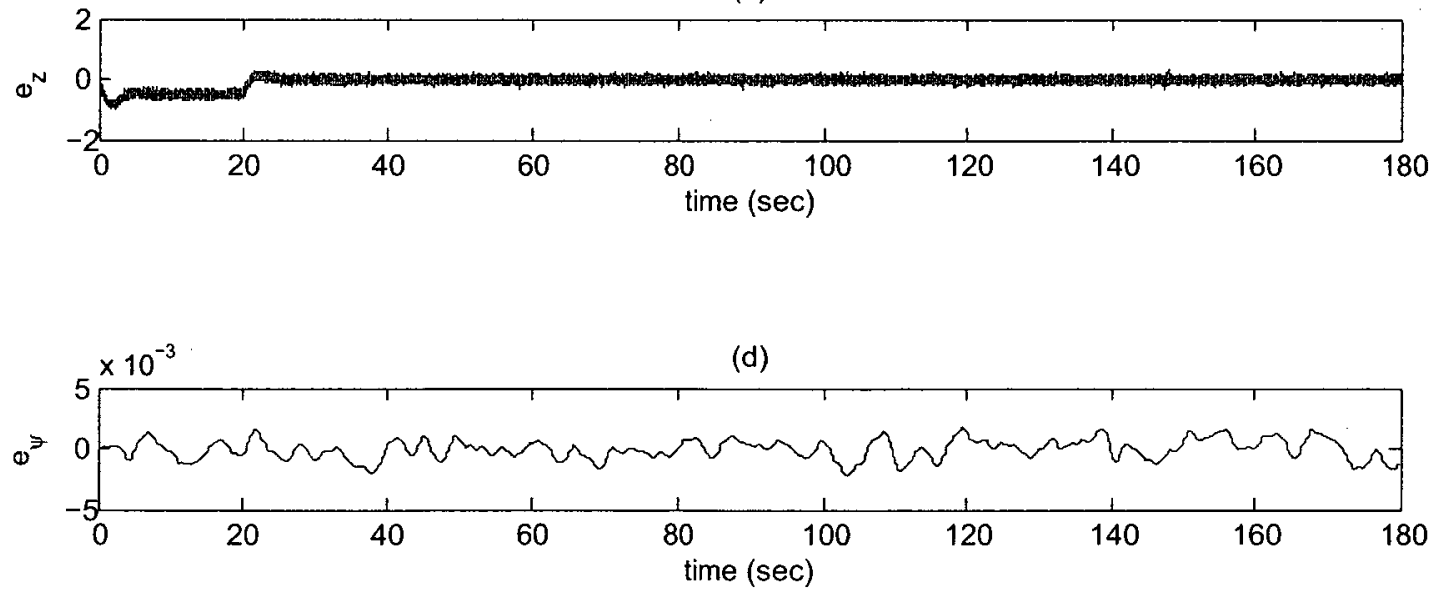

Figure 3.7: Error signals $e_{x}, e_{y}, e_{z}$ and $e_{\psi}$ measured in meters and $e_{\psi}$ in radian in response to the commanded 1st trajectory with $10 \%$ variations in the body inertia parameters. 
(a)

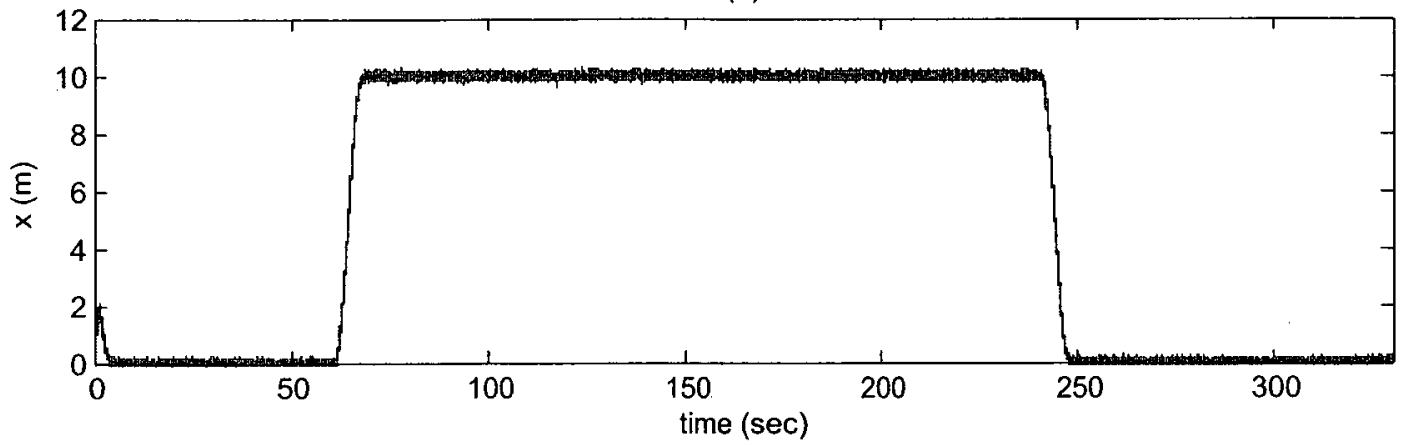

(b)

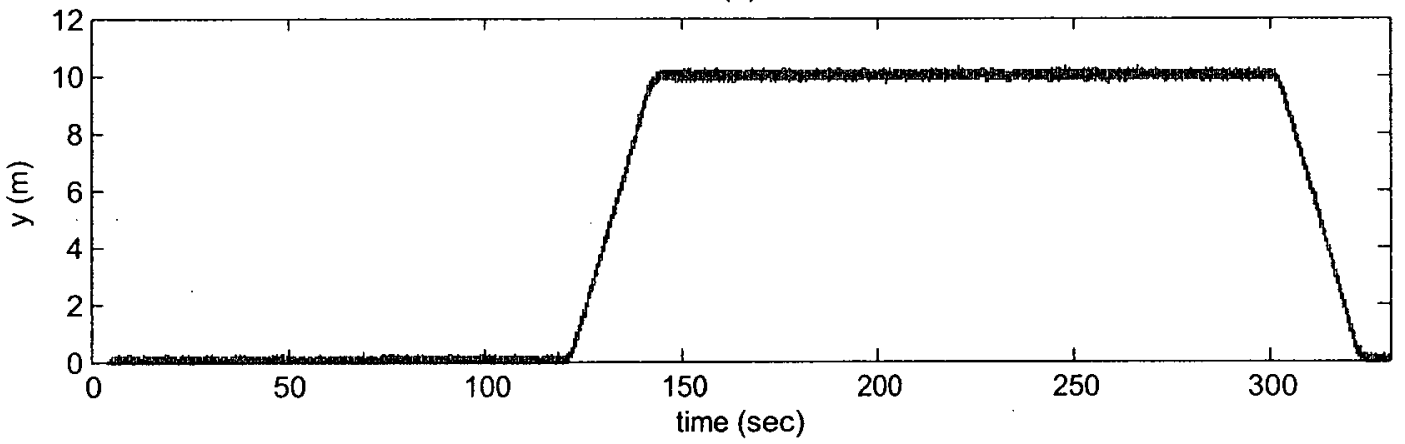

(c)

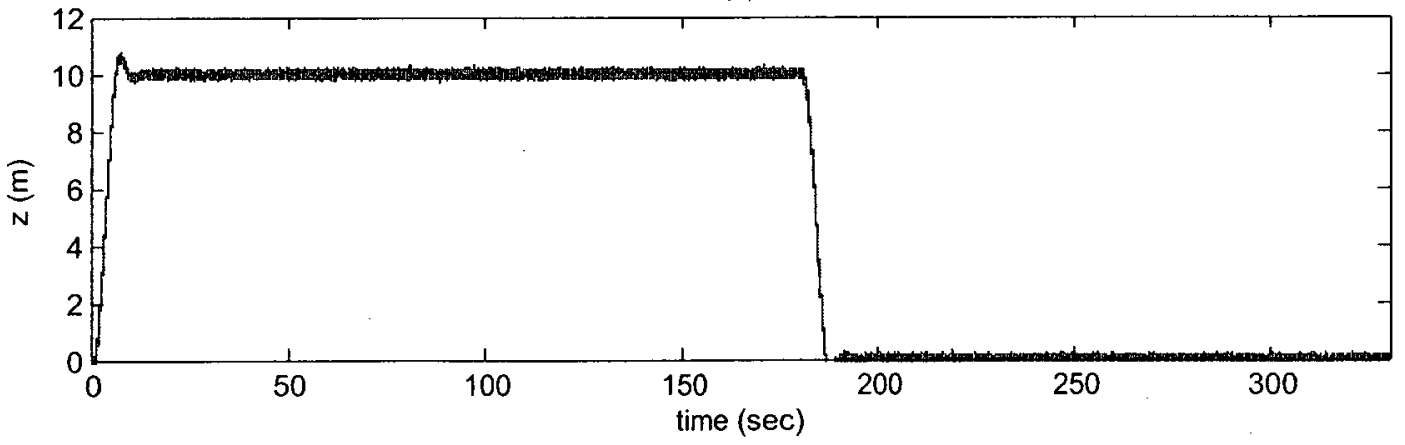

Figure 3.8: Linear position response to the commanded 2nd trajectory : (a) $x$, (b) $y$ and (c) $z$ measured in meters. 


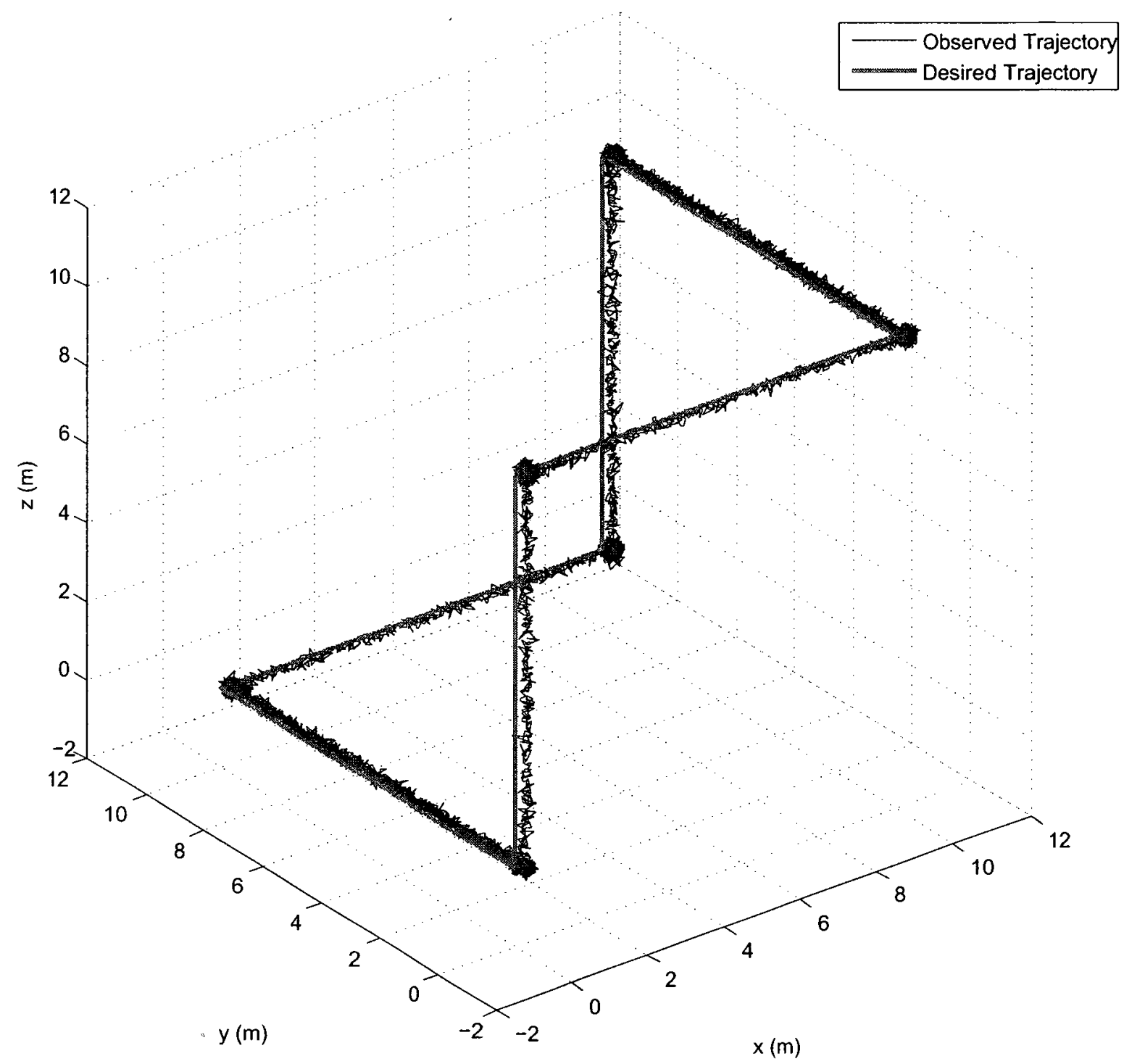

Figure 3.9: 3 -D representation of the linear position response to the commanded 2nd trajectory. 
(a)

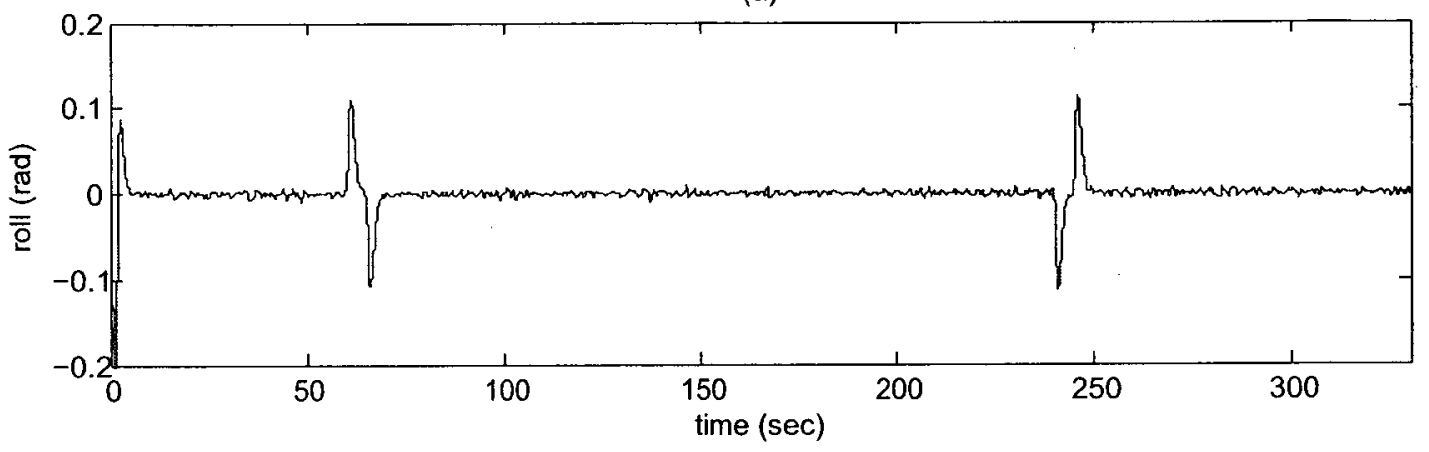

(b)
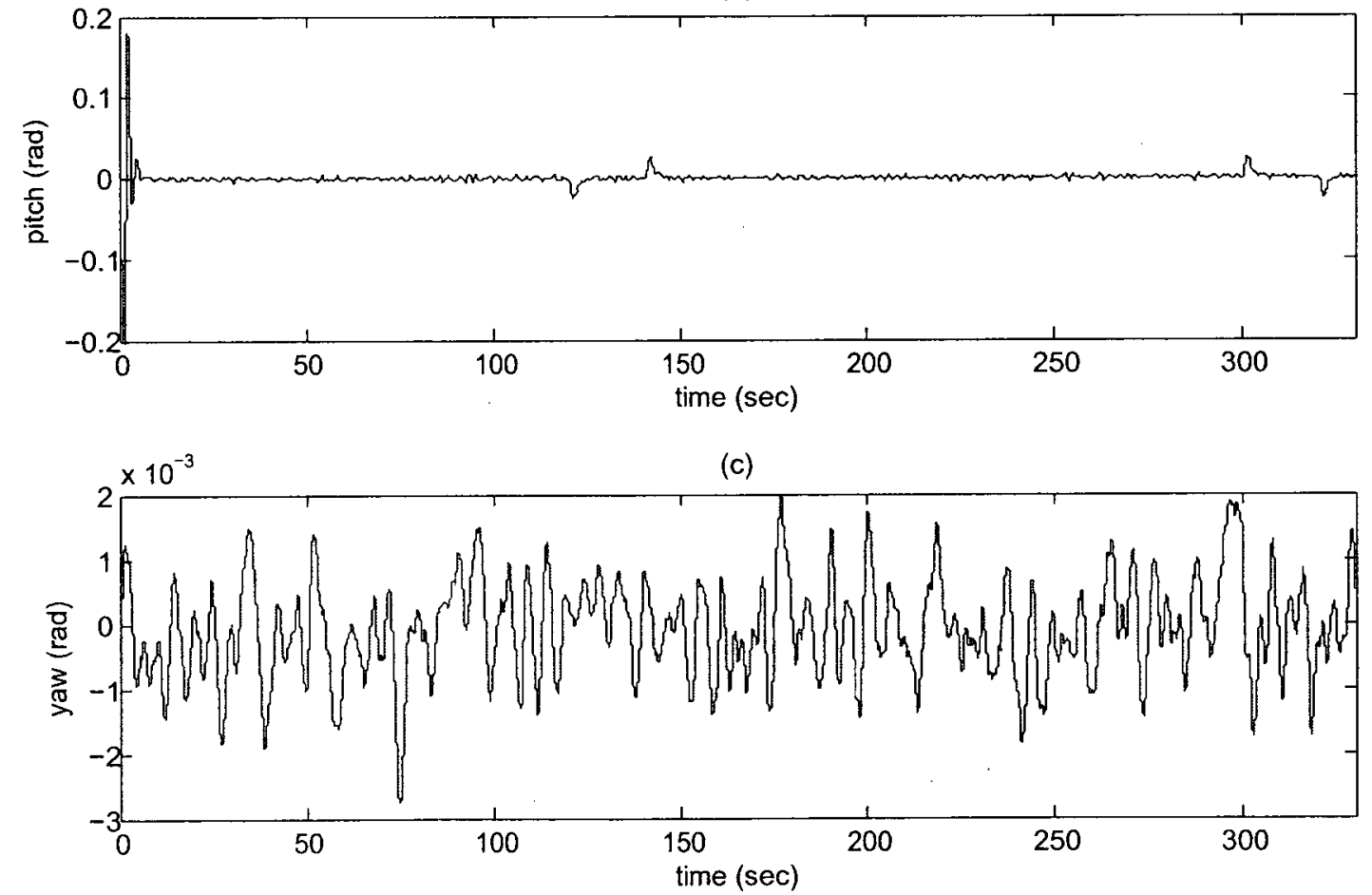

Figure 3.10: Euler angles in response to the commanded 2nd trajectory : (a) Roll (rad), (b) Pitch (rad) and (c) Yaw (rad). 
(a)

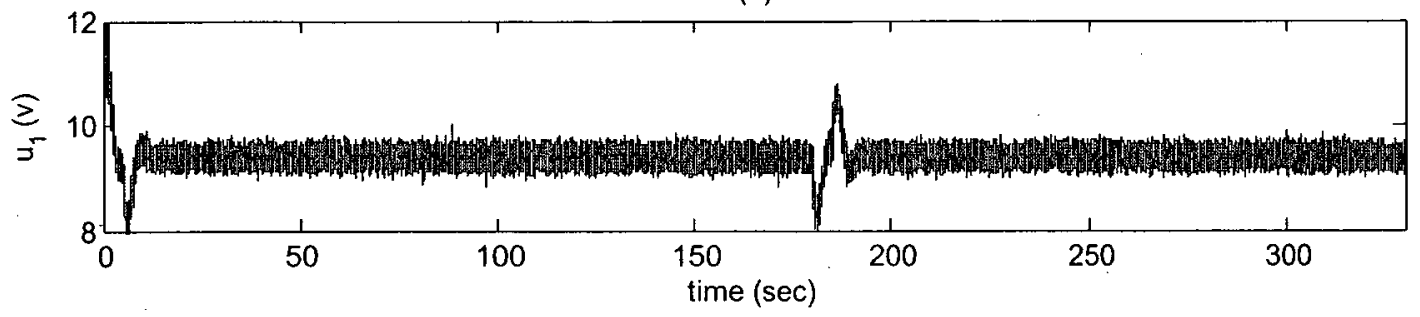

(b)

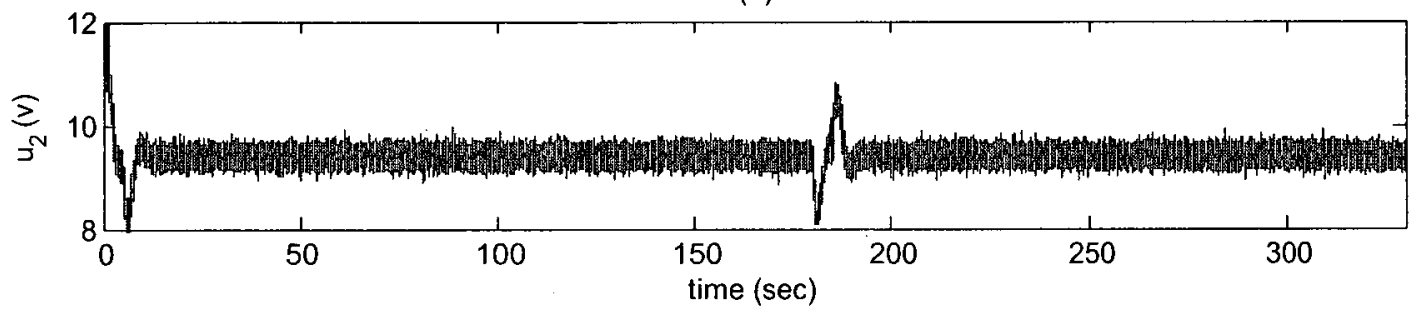

(c)

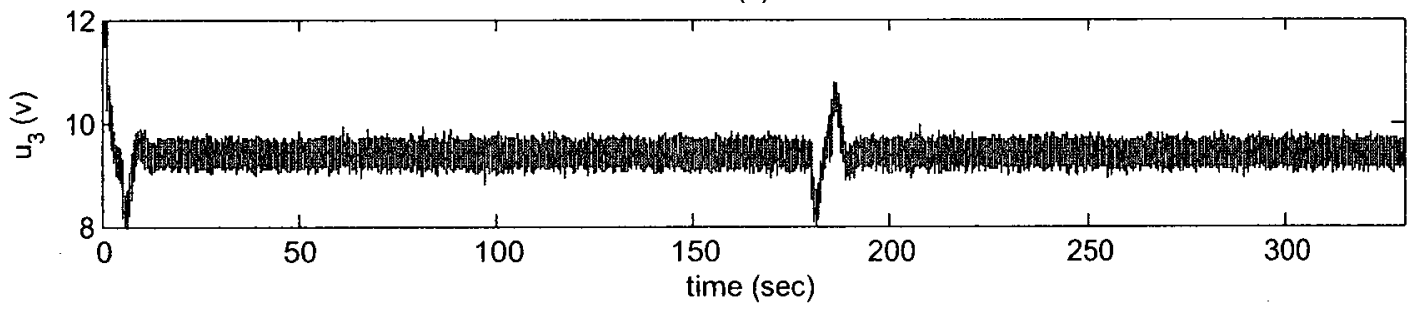

(d)

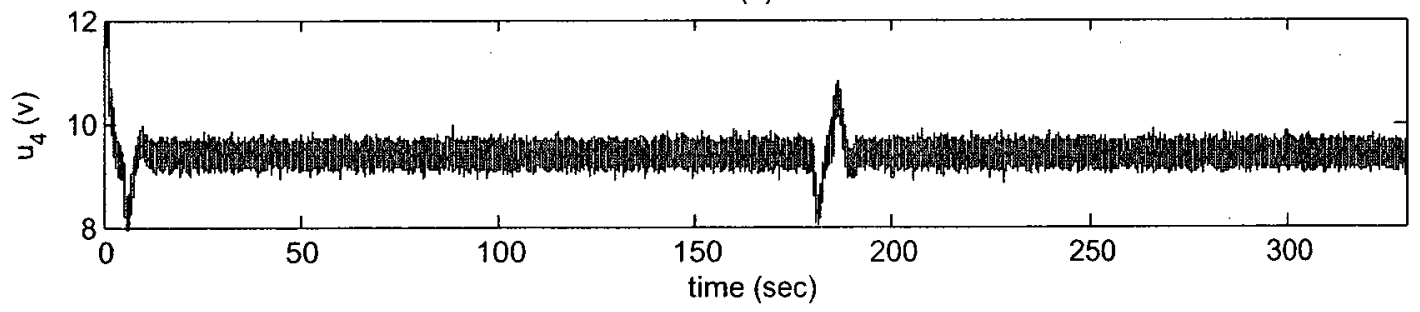

Figure 3.11: Input voltage to the 4 propellers in response to the commanded 2nd trajectory. 
(a)
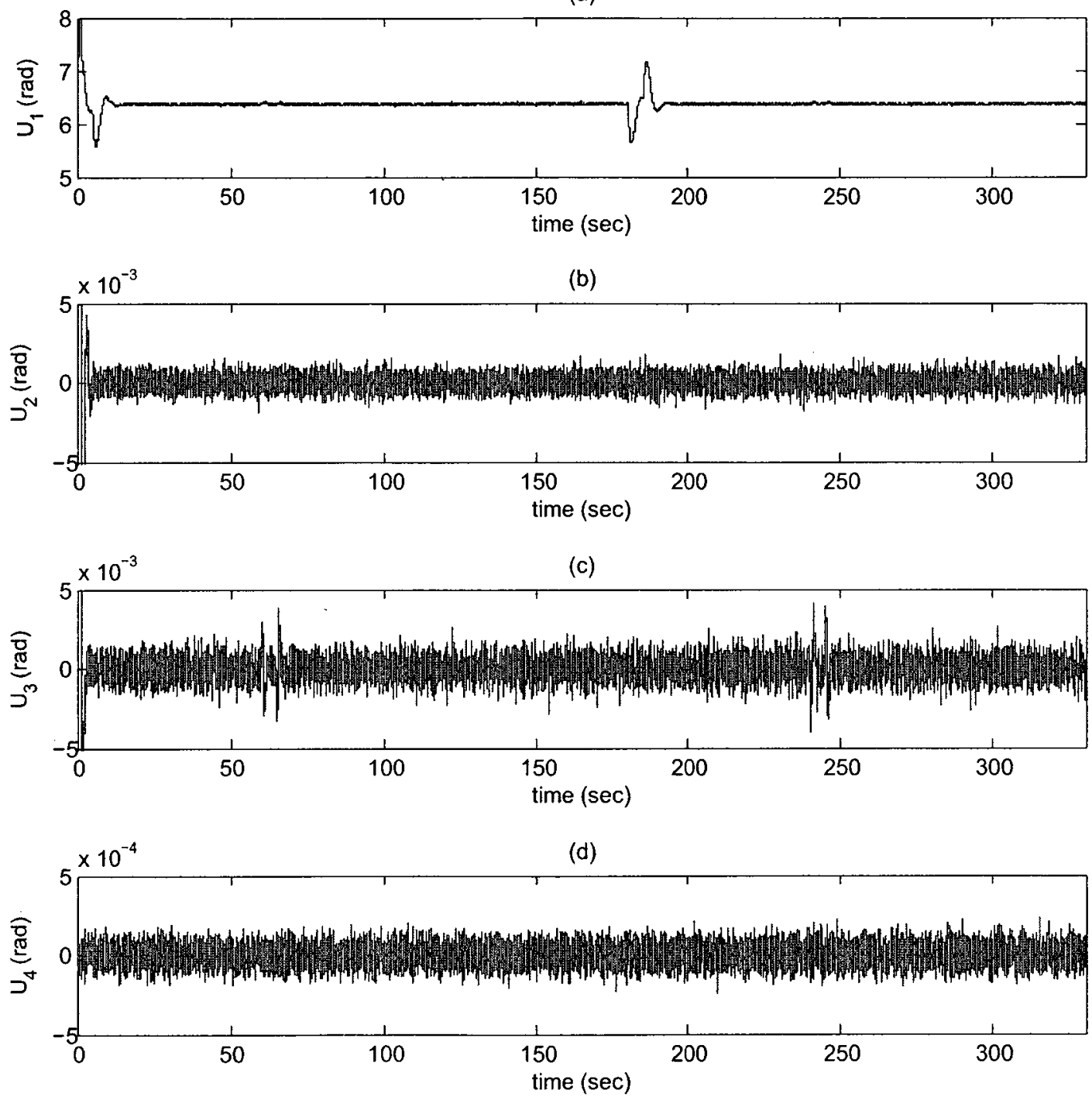

Figure 3.12: Input moments $U_{1}, U_{2}, U_{3}$ and $U_{4}$ to the dynamics of the quadrotor system in response to the commanded 2 nd trajectory. 
(a)

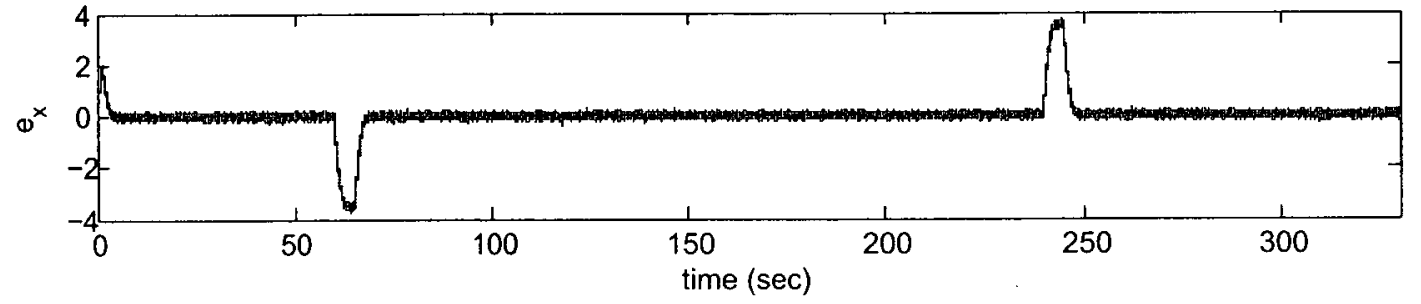

(b)

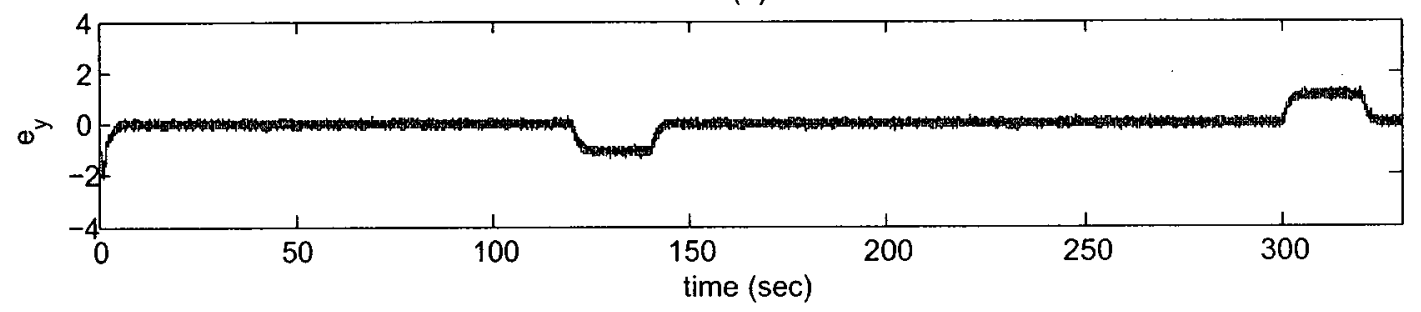

(c)
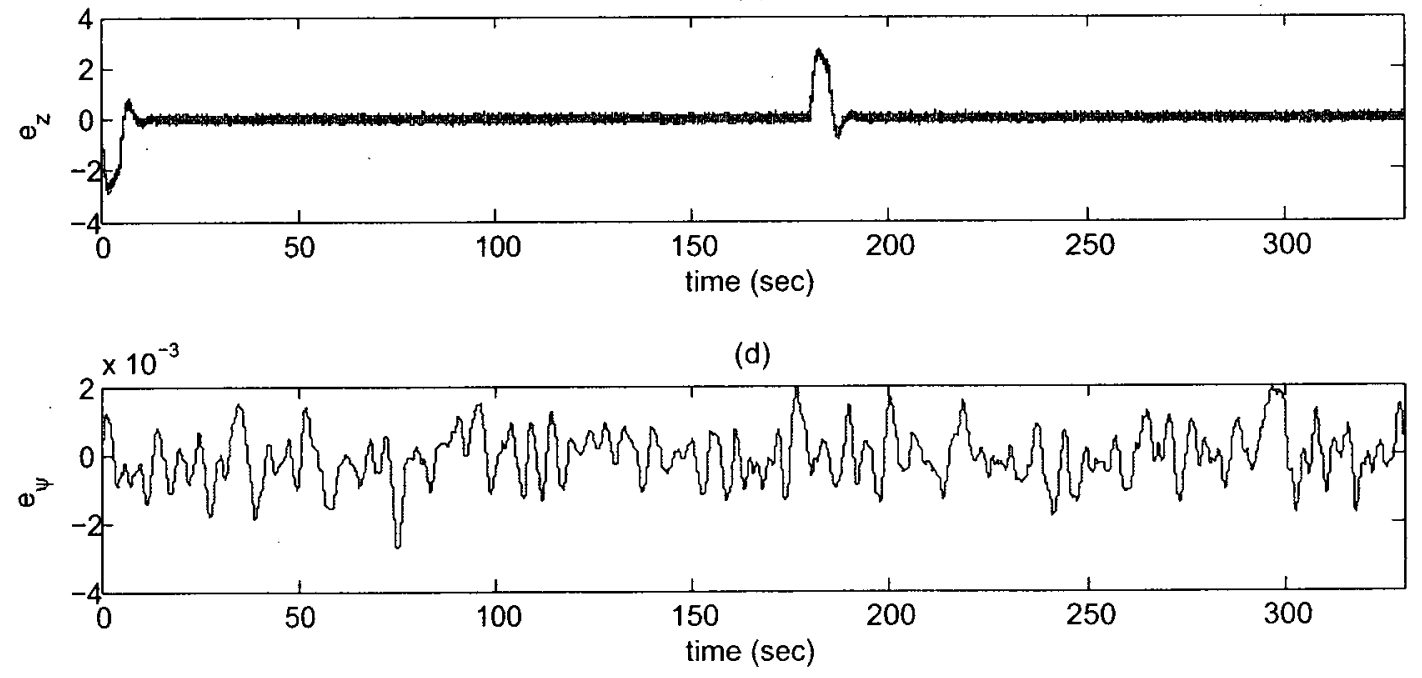

Figure 3.13: Error signals $e_{x}, e_{y}, e_{z}$ measured in meters and $e_{\psi}$ in radian in response to the commanded 2nd trajectory. 


\section{Chapter 4}

\section{Fault Recovery of the Quadrotor}

\section{System}

This chapter presents the results that are employed to develop a recovery solution due to actuator faults. The first section of this chapter will describe the general representation of the types of faults that might affect the actuators. The second section is dedicated to modeling of partial loss of effectiveness (LOE) fault in an actuator. The third section describes implementation of the fault recovery system. The above sections are dedicated to describe the proposed fault recovery algorithm by designing an adaptive feedback linearization controller. The final section presents simulation results performed to analyze the performance of the designed fault recovery module.

\subsection{Faults in the Quadrotor Actuators}

Dynamical control systems may be subject to faults in actuators, sensors or abrupt changes in the physical structure. This work is focused on the study of failures in actuators. In this section we present the parameterization of faults, which covers several different cases due to control effector faults.

Typical Actuator faults are classified into four categories [6]: 
- Lock-in-place (LIP)

- Float

- Hard-over-fault (HOF).

- Partial loss of effectiveness (LOE)

In the case of LIP faults the actuator freezes at a certain condition and does not respond to subsequent commands. HOF is characterized by the effector moving to the upper or lower position limit regardless of the command. Float fault occurs when the actuator floats with zero moment and does not contribute to the control authority. Loss of effectiveness is characterized by lowering the actuator gain with respect to its nominal value.

The parameterization of different types of actuator faults is given by

$$
y_{i}(t)=\left\{\begin{array}{lr}
y_{c i}(t), & k_{i i}(t)=1, \forall t>t_{0}, \quad \text { No fault } \\
k_{i}(t) y_{c i}(t), & 0<\epsilon \leq k_{i}(t)<1, \forall t \geq t_{f i}, \quad \text { LOE } \\
0, & k_{i}(t)=0, \forall t \leq t_{f i}, \quad \text { Float } \\
\bar{y}_{c i}\left(t_{f}\right), & k_{i}(t)=0, \forall t \leq t_{f i}, \quad \text { LIP } \\
y_{i m} \text { or } y_{i M}, & k_{i}(t)=0, \forall t \leq t_{f i}, \quad \text { HOF }
\end{array}\right.
$$

where $y_{i}$ is the actual output of the actuator, $y_{c i}$ is the output of the controller and $y_{i m}$ and $y_{i M}$ are the upper and lower limit, $k_{i} \in(\epsilon, 1]$ denotes the actuator effectiveness coefficient and models partial LOE fault and $\epsilon \ll 1$ and $t_{f i}$ also denotes the time instant of fault occurrence in the $i^{\text {th }}$ actuator. In the case of LIP fault, $\bar{y}_{c i}$ is a value within the range of operation of the $i^{\text {th }}$ actuator frozen at the time of failure $\left(t_{f_{i}}\right)$.

As discussed in the previous chapters, the quadrotor has 4 rotors as actuators. In Chapter 2 the thrust force was introduced as: 


$$
T_{i}=b \Omega_{i}^{2} \quad i=1, \ldots, 4
$$

where $\Omega_{i}\left(\mathrm{rads}^{-1}\right)$ is the speed of the the $i^{\imath} h$ propeller. In Chapter 3 the linearized dynamic equation from the input voltage to the $i^{i h}$ propeller, $u_{i}$, to the thrust $T_{i}$ was obtained as

$$
\dot{T}_{i}=A_{t} T_{i}+B_{t} u_{i}+C_{t}
$$

where $A_{t}, B_{t}$ and $C_{t}$ are the linearized coefficients and are defined as follows:

$$
\left\{\begin{array}{l}
A_{t}=\frac{-2}{\tau_{t}}-\frac{3 d}{\sqrt{b}} \sqrt{T_{0}} \\
B_{t}=\frac{\sqrt{b}}{k_{e} r \tau_{t}} \sqrt{T_{0}} \\
C_{t}=\frac{d}{\sqrt{b}} T_{0}^{\frac{3}{2}}
\end{array}\right.
$$

where $\frac{1}{\tau_{\iota}}=\frac{k_{t}^{2}}{R_{\text {mot }} J_{\iota p}} \eta r^{2}$. In the above equation $d$ and $b$ refer to the aerodynamic drag and thrust factors $\left(N m s^{2}\right), J_{i p}\left(N m s^{2}\right)$ is the total rotational inertia around the propeller axis, $k_{e}\left(N m A^{-1}\right)$ is the back-EMF constant, $R_{m o t}$ is the motor resistance in ohms, $\eta$ is the efficiency factor and $r$ refers to the gear box reduction ratio.

In the healthy model of the system we assume that all the four thrust torques $T_{i}$, for $i=1,2,3,4$, which are proportional to the square of propellers speeds, are equal and share the same equal parameters.

Considering $T=\left[\begin{array}{llll}T_{1} & T_{2} & T_{3} & T_{4}\end{array}\right]^{T}$ as the thrust vector, the compact representation of the dynamics becomes

$$
\dot{T}=-A_{T} T+B_{T} \underline{u}+C_{T}
$$

where $A_{T}=A_{t} I_{(4 \times 4)}$ and $B_{T}=B_{t} I_{(4 \times 4)}$ are constant matrices and $C_{T}=C_{t}\left[\begin{array}{llll}1 & 1 & 1 & 1\end{array}\right]^{T}$ is a constant vector. The notation $I_{(4 \times 4)}$ refers to an identity matrix of the dimension 
4 and $\underline{u}=\left[\begin{array}{llll}u_{1} & u_{2} & u_{3} & u_{4}\end{array}\right]^{T}$ is the vector of the input voltages to the propellers.

Quadrotor is an under-actuated system and there is no redundant actuator to compensate for the effects of fault due to other actuator failure. Therefore, in case of other type of faults, where one of the actuators is either stuck in a certain speed with no possibility of controlling as in the LIP and HOF or fully nonfunctional as in the float, the other three remaining healthy actuators should be commanded to compensate the loss of control on the faulty actuator. In this work, our concentration is on the recovery process due to the partial LOE fault, where all the actuators are still functional, however, a short discussion on the other types of faults is also provided in Section 4.5

\subsection{Partial LOE Fault Modeling}

In case of a partial LOE fault, the output speed of the quadrotor is different from the commanded output by the controller, that is

$$
\Omega_{i}=k_{i} \Omega_{c i} \quad 0<\epsilon<k_{i}<1
$$

where $\Omega_{i}$ refers to the actual output from the $i^{\text {th }}$ actuator and $\Omega_{c i}$ is the commanded output by the controller. Therefore, the resulting thrust force from this actuator varies according to the following equation

$$
\begin{aligned}
T_{i} & =b \Omega_{i}^{2} \\
& =b\left(k_{i} \Omega_{c i}\right)^{2}
\end{aligned}
$$

The dynamics of $T_{i}$ defined in equation (4.3) would also change due to the LOE fault, that is 


$$
\begin{aligned}
\dot{T}_{i} & =2 b k_{i}^{2} \Omega_{c i} \dot{\Omega}_{c i} \\
& =k_{i}^{2} A_{t}+k_{i}^{2} B_{t} \dot{u}_{i}+k_{i}^{2} C_{t}
\end{aligned}
$$

or in other words,

$$
\dot{T}_{i}=A_{t i} T_{i}+B_{t i} u_{i}+C_{t} \quad i=1,2,3,4
$$

where $A_{t i}=k_{i}^{2} A_{t}$ and $B_{t i}=k_{i}^{2} B_{t}$. It should be noted that we have assumed that the only coefficients subject to change due to a fault are the $A_{t i}$ and $B_{t i}$ and $C_{t}$ would stay unaffected. The term $C_{t}$ is proportional to the drag and inverse square of the thrust factor, which makes it a relatively small constant value.

Equation (4.10) is a matrix form of equation (4.9), that is

$$
\dot{T}=A_{T 0} T+B_{T 0} \underline{u}+C_{T}
$$

where

$$
\begin{aligned}
& A_{T 0}=\left[\begin{array}{cccc}
A_{t 1} & 0 & 0 & 0 \\
0 & A_{t 2} & 0 & 0 \\
0 & 0 & A_{t 3} & 0 \\
0 & 0 & 0 & A_{t 4}
\end{array}\right] \\
& B_{T 0}=\left[\begin{array}{cccc}
B_{t 1} & 0 & 0 & 0 \\
0 & B_{t 2} & 0 & 0 \\
0 & 0 & B_{t 3} & 0 \\
0 & 0 & 0 & B_{t 4}
\end{array}\right]
\end{aligned}
$$




$$
C_{T}=\left[\begin{array}{cccc}
C_{t} & 0 & 0 & 0 \\
0 & C_{\iota} & 0 & 0 \\
0 & 0 & C_{\iota} & 0 \\
0 & 0 & 0 & C_{t}
\end{array}\right]
$$

Now if the thrust dynamics for all the actuators are not equal, the equations for the dynamics of the movement vector $U$ change since $A_{T} \neq A_{t} I$ and $B_{T} \neq B_{t} I$. Therefore, it is necessary to derive the dynamic equations for the movement vector while the actuators do not have the same characteristics, in other words when $A_{t i} \neq$ $A_{t j}$ and $B_{t i} \neq B_{t j}$ for $i, j=1, \ldots, 4, i \neq j$.

The relation between the movement vector $U$ and $T$ was defined as in equation (3.11). By left-multiplying $L_{U T}$ to equation (4.10), we would have

$$
L_{U T} \dot{T}=-\left(L_{U T} A_{T 0}\right) T+\left(L_{U T} B_{T 0}\right) \underline{u}+\left(L_{U T} C_{T}\right)
$$

From equation (3.11), the equation (4.14) could be rewritten as (4.15), namely

$$
\dot{U}=-\left(L_{U T} A_{T 0} L_{U T}^{-1}\right) U+\left(L_{U T} B_{T 0}\right) \underline{u}+\left(L_{U T} C_{T}\right)
$$

Now we define another new input vector $\underline{v_{0}}$ as

$$
\begin{gathered}
\underline{v_{0}}=\left(L_{U T} B_{T 0}\right) \underline{u}=\left[\begin{array}{c}
v_{01} \\
v_{02} \\
v_{03} \\
v_{04}
\end{array}\right]=\left[\begin{array}{cccc}
B_{t 1} & B_{t 2} & B_{t 3} & B_{l 4} \\
0 & -l B_{t 2} & 0 & B_{t 4} \\
-l B_{t 1} & 0 & l B_{t 3} & B_{t 4} \\
-\frac{d}{b} B_{t 1} & \frac{d}{b} B_{t 2} & -\frac{d}{b} B_{t 3} & \frac{d}{b} B_{t 4}
\end{array}\right]\left[\begin{array}{l}
u_{1} \\
u_{2} \\
u_{3} \\
u_{4}
\end{array}\right] . \\
\underline{u}=\left(L_{U T} B_{T 0}\right)^{-1} \underline{v_{0}}
\end{gathered}
$$


The matrix $\left(L_{U T} A_{T 0} L_{U T}^{-1}\right)$ is given by

$$
\begin{aligned}
& L_{U T} A_{T 0} L_{U T}^{-1}= \\
& {\left[\begin{array}{cccc}
\frac{1}{4}\left(A_{t 1}+A_{t 2}+A_{t 3}+A_{t 4}\right) & \frac{1}{2 l}\left(-A_{t 2}+A_{t 4}\right) & \frac{1}{2}\left(-A_{t 1}+A_{t 3}\right) & \frac{b}{4 d}\left(-A_{t 1}+A_{t 2}-A_{t 3}+A_{t 4}\right) \\
\frac{l}{4}\left(-A_{t 2}+A_{t 4}\right) & \frac{1}{2}\left(A_{t 2}+A_{t 4}\right) & 0 & \frac{l b}{4 d}\left(-A_{t 2}+A_{t 4}\right) \\
\frac{l}{4}\left(-A_{t 1}+A_{t 3}\right) & 0 & \frac{1}{2}\left(A_{t 1}+A_{t 3}\right) & \frac{l b}{4 d}\left(A_{t 1}-A_{t 3}\right) \\
\frac{d}{4 b}\left(-A_{t 1}+A_{t 2}-A_{t 3}+A_{t 4}\right) & \frac{d}{2 l b}\left(-A_{t 2}+A_{t 4}\right) & \frac{d}{2 l b}\left(A_{t 1}-A_{t 3}\right) & \frac{1}{4}\left(A_{t 1}+A_{t 2}+A_{t 3}+A_{t 4}\right)
\end{array}\right]}
\end{aligned}
$$

From equation (4.18), it is obvious that if the actuators share the same parameters , in other words $A_{t i}=A_{t}$ and $B_{t i}=B_{t}$ for $i=1, \ldots, 4$, then $\left(L_{U T} A_{T 0} L_{U T}^{-1}\right)=A_{T}=$ $A_{t} I_{4 \times 4}$ as expected for the healthy system.

Hence, it is possible to rewrite equation (4.15) as

$$
\dot{U}=-\left(L_{U T} A_{T 0} L_{U T}^{-1}\right) U+\underline{v_{0}}+\left(L_{U T} C_{T}\right)
$$

The above equation shows the dynamics of the movement vector $U$, while the dynamics of the thrust forces are considered to be different from each other due to the presence of LOE fault.

\subsection{Implementation of a Fault Recovery System}

Figure (4.1) depicts the modules that conform the fault recovery system developed in this chapter. The descriptions of each component of the quadrotor control system with fault recovery for actuator faults are provided below.

- Linear Compensator: This corresponds to assigning the control input for the purpose of tracking the desired trajectory for the system according to an LQR design. 


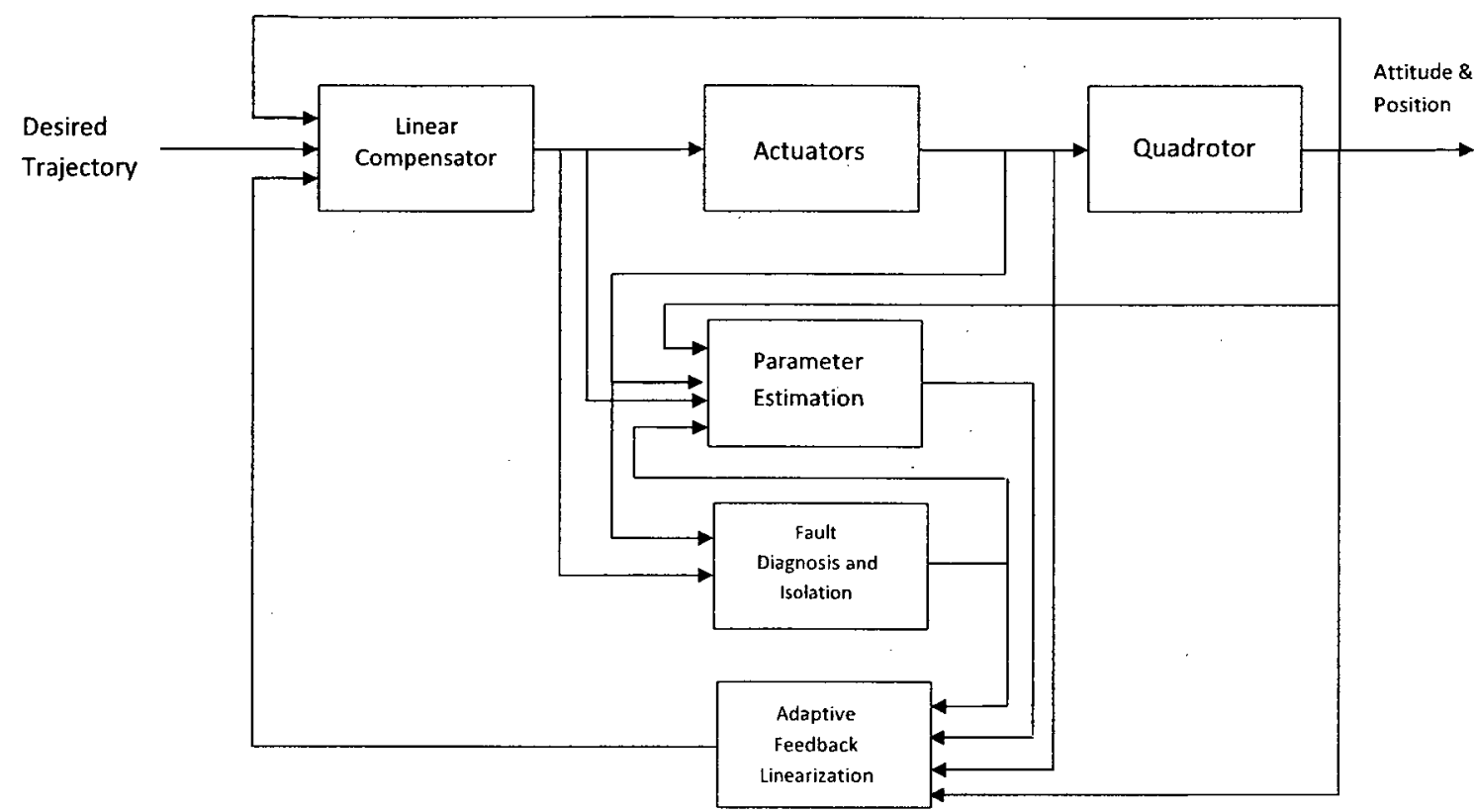

Figure 4.1: Quadrotor augmented control system with a fault recovery functionality.

- Actuators: This block contains the model of the actuators, where the input is the voltage to the rotors and the output is in terms of the thrust force due to the rotation of the propellers.

- Fault Diagnosis and Isolation (FDI): Is in charge of generating fault detection alarms and information about the location and type of the fault.

- Parameter Estimation: Given the information from the system input and output and the FDI information, this module is in charge of generating an estimate of the post fault model of the actuator to be used by the feedback linearization scheme.

- Adaptive Feedback Linearization : Receiving the information from the FDI unit as well as the parameter estimation unit and the current measured (or estimated) values of the outputs or states of the quadrotor system, this block is in charge of generating a control input to linearize the system adaptively. In 
the no-fault case, this block generates the control input for the healthy system.

- Quadrotor : The full nonlinear model of the dynamics and kinematics equations of motion of the quadrotor are placed in this block.

\subsection{Fault Recovery Module}

The recovery strategy in this work is to adapt the control inputs so that the effects of changes in the outputs due to the LOE fault in an actuator can be compensated for. Therefore, it is necessary to analyze the contribution of each actuator output in terms of the thrust force in the control of the system outputs.

\subsubsection{Adaptive Feedback Linearization}

At the end of Section 4.1, the dynamic equations of movement vector were derived in equation (4.19). In this equation the contribution of each actuator in the resulting movement vector was obtained. As discussed before, in case of the partial LOE fault occurrence the parameters of the actuators may change. A parameter estimation algorithm is presented in this section to provide an estimate of the faulty actuator dynamics and to guarantee the stability of the closed-loop system.

Moreover, a controller based on adaptive feedback linearization technique is designed by considering the dynamic equation of the movement vector obtained in equation (4.19).

In Chapter 3, Section 3.3.3 the following equations were derived while designing the FL controller for $\left(z, U_{1}\right),\left(y, U_{2}\right),\left(x, U_{3}\right)$ and $\left(\phi, U_{4}\right)$ subsystems, namely

$$
z^{(3)}=-\dot{\phi} \sin \phi \cos \theta \frac{U_{1}}{m}-\dot{\theta} \cos \phi \sin \theta \frac{U_{1}}{m}+\cos \phi \cos \theta \frac{\dot{U}_{1}}{m}
$$




$$
\begin{gathered}
y^{(5)}=-g \frac{\dot{U}_{2}}{I_{x x}} \cos \phi+g \frac{U_{2}}{I_{x x}} \dot{\phi} \sin \phi+2 g \dot{\phi} \ddot{\phi} \sin \phi+g \dot{\phi}^{3} \cos \phi \\
x^{(5)}=g \frac{\dot{U}_{3}}{I_{y y}} \cos \theta-g \frac{U_{3}}{I_{y y}} \dot{\theta} \sin \theta-2 g \ddot{\theta} \ddot{\theta} \sin \theta-g \dot{\theta}^{3} \cos \theta \\
\psi^{(3)}=\frac{\dot{U}_{4}}{I_{z z}}
\end{gathered}
$$

Without loss of generality, let us assume that the partial LOE fault has occurred in the first actuator and the other three actuators are healthy, that is

$$
\left\{\begin{array}{l}
\dot{T}_{1}=A_{t 1} T_{1}+B_{t 1} u_{1}+C_{t} \\
\dot{T}_{i}=A_{t} T_{i}+B_{t} u_{i}+C_{t} \quad \text { for } \quad \mathrm{i}=2,3,4
\end{array}\right.
$$

where $A_{t 1}=k^{2} A_{t}$ and $B_{t 1}=k^{2} B_{t}$.

The dynamics of the movement vector defined in (4.15) can be written as

$$
\begin{aligned}
& {\left[\begin{array}{c}
\dot{U}_{1} \\
\dot{U}_{2} \\
\dot{U}_{3} \\
\dot{U}_{4}
\end{array}\right]=\left[\begin{array}{cccc}
\frac{1}{4}\left(A_{t 1}+3 A_{t}\right) & 0 & \frac{1}{2}\left(-A_{t 1}+A_{t}\right) & \frac{b}{4 d}\left(-A_{t 1}+A_{t}\right) \\
0 & A_{t} & 0 & 0 \\
\frac{l}{4}\left(-A_{t 1}+A_{t}\right) & 0 & \frac{1}{2}\left(A_{t 1}+A_{t}\right) & \frac{l b}{4 d}\left(A_{t 1}-A_{t}\right) \\
\frac{d}{4 b}\left(-A_{t 1}+A_{t}\right) & 0 & \frac{d}{2 l b}\left(A_{t 1}-A_{t}\right) & \frac{1}{4}\left(A_{t 1}+3 A_{t}\right)
\end{array}\right]\left[\begin{array}{c}
U_{1} \\
U_{2} \\
U_{3} \\
U_{4}
\end{array}\right] } \\
&+ {\left[\begin{array}{cccc}
B_{t 1} & B_{t} & B_{t} & B_{t} \\
0 & -l B_{t} & 0 & B_{t} \\
-l B_{t 1} & 0 & l B_{t} & 0 \\
-\frac{d}{b} B_{t 1} & \frac{d}{b} B_{t} & -\frac{d}{b} B_{t} & \frac{d}{b} B_{t}
\end{array}\right]\left[\begin{array}{c}
u_{1} \\
u_{2} \\
u_{3} \\
u_{4}
\end{array}\right]+\left(L_{U T} C_{T}\right) }
\end{aligned}
$$

The above dynamic equation is then substituted in the equations (4.20) to (4.23) and could be rewritten in a compact matrix form. Here we are seeking a form 
to separate the terms that are related to the unknown variables $A_{t 1}$ and $B_{t 1}$. This is done in the following equation as

$$
\left[\begin{array}{c}
z^{(3)} \\
y^{(5)} \\
x^{(5)} \\
\psi^{(3)}
\end{array}\right]=F_{1}+A_{t 1} F_{2}+F_{3}\left[\begin{array}{cccc}
B_{t 1} & 0 & 0 & 0 \\
0 & B_{t} & 0 & 0 \\
0 & 0 & B_{t} & 0 \\
0 & 0 & 0 & B_{t}
\end{array}\right]\left[\begin{array}{c}
u_{1} \\
u_{2} \\
u_{3} \\
u_{4}
\end{array}\right]
$$

where

$$
\begin{aligned}
& F_{1}=\left[\begin{array}{c}
-\dot{\phi} \sin \phi \cos \theta \frac{U_{1}}{m}-\dot{\theta} \cos \phi \sin \theta \frac{U_{1}}{m}+\frac{\cos \phi \cos \theta}{m} A_{t}\left(\frac{3}{4} U_{1}+\frac{1}{2} U_{3}+\frac{b}{4 d} U_{4}\right)+4 C_{t} \\
3 g \frac{U_{2}}{I_{x x}} \dot{\phi} \sin \phi+g \dot{\phi}^{3} \cos \phi-\frac{g}{I_{x x}} \cos \phi A_{t} U_{2} \\
-3 g \frac{U_{3}}{I_{y y}} \dot{\theta} \sin \theta-g \dot{\theta}^{3} \cos \theta+\frac{g \cos \theta}{I_{y y}} A_{t}\left(\frac{l}{4} U_{1}+\frac{1}{2} U_{3}-\frac{l b}{4 d} U_{4}\right) \\
\frac{1}{I_{z z}} A_{t}\left(\frac{d}{4 b} U_{1}-\frac{d}{2 l b} U_{3}+\frac{3}{4} U_{4}\right)
\end{array}\right] \\
& F_{2}=\left[\begin{array}{c}
\frac{\cos \phi \cos \theta}{m}\left(\frac{1}{4} U_{1}-\frac{1}{2} U_{3}-\frac{b}{4 d} U_{4}\right) \\
0 \\
\frac{g \cos \theta}{I_{y y}}\left(-\frac{l}{4} U_{1}+\frac{1}{2} U_{3}+\frac{l b}{4 d} U_{4}\right) \\
\frac{1}{I_{z z}} A_{t}\left(-\frac{d}{4 b} U_{1}+\frac{d}{2 l b} U_{3}+\frac{1}{4} U_{4}\right)
\end{array}\right] \\
& F_{3}=\left[\begin{array}{cccc}
\frac{\cos \phi \cos \theta}{m} & \frac{\cos \phi \cos \theta}{m} & \frac{\cos \phi \cos \theta}{m} & \frac{\cos \phi \cos \theta}{m} \\
0 & \frac{g \cos \phi}{I_{x x}} l & 0 & -\frac{g \cos \phi}{I_{x x}} l \\
-\frac{g \cos \theta}{I_{y y}} l & 0 & \frac{g \cos \theta}{I_{y y}} l & 0 \\
\frac{1}{I_{z z}}\left(-\frac{d}{b}\right) & \frac{1}{I_{z z}}\left(\frac{d}{b}\right) & \frac{1}{I_{z z}}\left(-\frac{d}{b}\right) & \frac{1}{I_{z z}}\left(\frac{d}{b}\right)
\end{array}\right]
\end{aligned}
$$

The input signal $u$ is defined as

$$
\left[\begin{array}{l}
u_{1} \\
u_{2} \\
u_{3} \\
u_{4}
\end{array}\right]=\left(F_{3}\left[\begin{array}{cccc}
\hat{B}_{t 1} & 0 & 0 & 0 \\
0 & B_{t} & 0 & 0 \\
0 & 0 & B_{t} & 0 \\
0 & 0 & 0 & B_{t}
\end{array}\right]\right)^{-1}\left(\left[\begin{array}{c}
w_{1} \\
w_{2} \\
w_{3} \\
w_{4}
\end{array}\right]-F_{1}-\hat{A}_{t 1} F_{2}\right)
$$


where $\hat{A}_{t 1}$ and $\hat{B}_{t 1}$ are the estimates of the unknown parameters $A_{t 1}$ and $B_{t 1}$ and the new control input vector $W=\left[\begin{array}{llll}w_{1} & w_{2} & w_{3} & w_{4}\end{array}\right]^{T}$ is to be defined so that the stability and control (LQR) of the feedback linearized system is achieved.

By applying the control law defined in (4.28) to the system in (4.26), the closed-loop dynamics could be written as

$$
\left[\begin{array}{l}
z^{(3)} \\
y^{(5)} \\
x^{(5)} \\
\psi^{(3)}
\end{array}\right]=F_{1}+A_{t 1} F 2+F 3\left[\begin{array}{cccc}
B_{t 1} & 0 & 0 & 0 \\
0 & B_{t} & 0 & 0 \\
0 & 0 & B_{t} & 0 \\
0 & 0 & 0 & B_{t}
\end{array}\right]\left[\begin{array}{cccc}
\hat{B}_{t 1} & 0 & 0 & 0 \\
0 & B_{t} & 0 & 0 \\
0 & 0 & B_{t} & 0 \\
0 & 0 & 0 & B_{t}
\end{array}\right]^{-1} F_{3}^{-1}\left(\left[\begin{array}{c}
w_{1} \\
w_{2} \\
w_{3} \\
w_{4}
\end{array}\right]_{(4.29)}-F_{1}-\hat{A}_{t 1} F_{2}\right)
$$

It is possible to simplify the above equation by using the following relation, that is

$$
\begin{aligned}
{\left[\begin{array}{cccc}
B_{t 1} & 0 & 0 & 0 \\
0 & B_{t} & 0 & 0 \\
0 & 0 & B_{t} & 0 \\
0 & 0 & 0 & B_{t}
\end{array}\right]\left[\begin{array}{cccc}
\hat{B}_{t 1} & 0 & 0 & 0 \\
0 & B_{t} & 0 & 0 \\
0 & 0 & B_{t} & 0 \\
0 & 0 & 0 & B_{t}
\end{array}\right]^{-1} } & =\left[\begin{array}{cccc}
\frac{B_{t 1}}{\hat{B}_{t 1}} & 0 & 0 & 0 \\
0 & 1 & 0 & 0 \\
0 & 0 & 1 & 0 \\
0 & 0 & 0 & 1
\end{array}\right] \\
& =\left[\begin{array}{cccc}
\frac{B_{t 1}-\hat{B}_{t 1}}{\hat{B}_{t 1}} & 0 & 0 & 0 \\
0 & 0 & 0 & 0 \\
0 & 0 & 0 & 0 \\
0 & 0 & 0 & 0
\end{array}\right]+\left[\begin{array}{cccc}
1 & 0 & 0 & 0 \\
0 & 1 & 0 & 0 \\
0 & 0 & 1 & 0 \\
0 & 0 & 0 & 1
\end{array}\right]
\end{aligned}
$$

Let us define the estimation error $\delta$ as the difference between the actual value of the unknown parameter and its estimate, i.e. $\delta_{A_{t 1}}=A_{t 1}-\hat{A}_{t 1}$ and $\delta_{B_{t 1}}=B_{t 1}-\hat{B}_{t 1}$. Therefore, equation (4.29) can be rewritten as 


$$
\begin{aligned}
& {\left[\begin{array}{l}
z^{(3)} \\
y^{(5)} \\
x^{(5)} \\
\psi^{(3)}
\end{array}\right]=F_{1}+A_{t 1} F 2+F_{3}\left[\begin{array}{cccc}
\frac{\delta_{B_{t 1}}}{\hat{B}_{t 1}} & 0 & 0 & 0 \\
0 & 0 & 0 & 0 \\
0 & 0 & 0 & 0 \\
0 & 0 & 0 & 0
\end{array}\right] F_{3}^{-1}\left(\left[\begin{array}{c}
w_{1} \\
w_{2} \\
w_{3} \\
w_{4}
\end{array}\right]-F_{1}-\hat{A}_{t 1} F_{2}\right)} \\
& +F_{3} \times F_{3}^{-1}\left(\left[\begin{array}{c}
w_{1} \\
w_{2} \\
w_{3} \\
w_{4}
\end{array}\right]-F_{1}-\hat{A}_{t 1} F_{2}\right) \\
& =\left[\begin{array}{l}
w_{1} \\
w_{2} \\
w_{3} \\
w_{4}
\end{array}\right]+\delta_{A_{t 1}} F_{2}+\delta_{B_{t 1}} F_{3}\left[\begin{array}{cccc}
\frac{1}{\hat{B}_{t 1}} & 0 & 0 & 0 \\
0 & 0 & 0 & 0 \\
0 & 0 & 0 & 0 \\
0 & 0 & 0 & 0
\end{array}\right] F_{3}^{-1}\left(\left[\begin{array}{c}
w_{1} \\
w_{2} \\
w_{3} \\
w_{4}
\end{array}\right]-F_{1}-\hat{A}_{t 1} F_{2}\right)
\end{aligned}
$$

Let

$$
F_{4}=F_{3}\left[\begin{array}{cccc}
\frac{1}{\hat{B}_{t 1}} & 0 & 0 & 0 \\
0 & 0 & 0 & 0 \\
0 & 0 & 0 & 0 \\
0 & 0 & 0 & 0
\end{array}\right] F_{3}^{-1}\left(\left[\begin{array}{c}
w_{1} \\
w_{2} \\
w_{3} \\
w_{4}
\end{array}\right]-F_{1}-\hat{A}_{t 1} F_{2}\right)
$$

Hence, the linearized dynamics of system (4.31) can be rewritten as

$$
\left[\begin{array}{l}
z^{(3)} \\
y^{(5)} \\
x^{(5)} \\
\psi^{(3)}
\end{array}\right]=\left[\begin{array}{c}
w_{1} \\
w_{2} \\
w_{3} \\
w_{4}
\end{array}\right]+\delta_{A_{t 1}} F_{2}+\delta_{B_{t 1}} F_{4}
$$

The control inputs $w_{1}, w_{2}, w_{3}$ and $w_{4}$ are defined according to the following 
equations

$$
\begin{gathered}
w_{1}=z_{d}^{(3)}-k_{1 z}\left(\ddot{e}_{z}\right)-k_{2 z}\left(\dot{e}_{z}\right)-k_{3 z}\left(e_{z}\right) \\
w_{2}=y_{d}^{(5)}-k_{1 y}\left(e_{y}^{(4)}\right)-k_{2 y}\left(e_{y}^{(3)}\right)-k_{3 y}\left(\ddot{e}_{y}\right)-k_{4 y}(\dot{e})_{y}-k_{5 y}\left(e_{y}\right) \\
w_{3}=x_{d}^{(5)}-k_{1 x}\left(e_{x}^{(4)}\right)-k_{2 x}\left(e_{x}^{(3)}\right)-k_{3 x}(\ddot{e})_{x}-k_{4 x}(\dot{e})_{x}-k_{5 x}\left(e_{x}\right) \\
w_{4}=\psi_{d}^{(3)}-k_{1 \psi}\left(\ddot{e}_{\psi}\right)-k_{2 \psi}\left(\dot{e}_{\psi}\right)-k_{3 \psi}\left(e_{\psi}\right)
\end{gathered}
$$

In the above equations, $z_{d}, y_{d}, x_{d}$ and $\psi_{d}$ refer to the desired output variables, $e_{z}=z-z_{d}, e_{y}=y-y_{d}$ and $e_{x}=x-x_{d}$ and $e_{\psi}=\psi-\psi_{d}$ are defined as error signals.

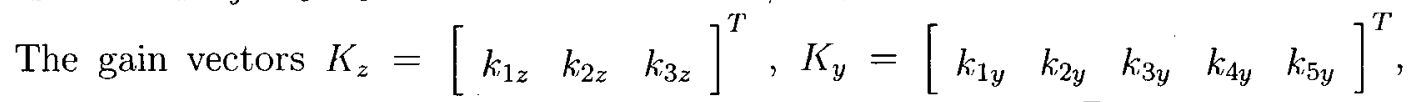
$K_{x}=\left[\begin{array}{lllll}k_{1 x} & k_{2 x} & k_{3 x} & k_{4 x} & k_{5 x}\end{array}\right]^{T}$ and $K_{\psi}=\left[\begin{array}{lll}k_{1 \psi} & k_{2 \psi} & k_{3 \psi}\end{array}\right]^{T}$ are obtained from the LQR method as described in Section 3.3.1.

From the equations (4.33) and (4.34) to (4.37), it is possible to write the dynamic equations of the error signals $e_{z}, e_{y}, e_{x}$ and $e_{\psi}$ as follows

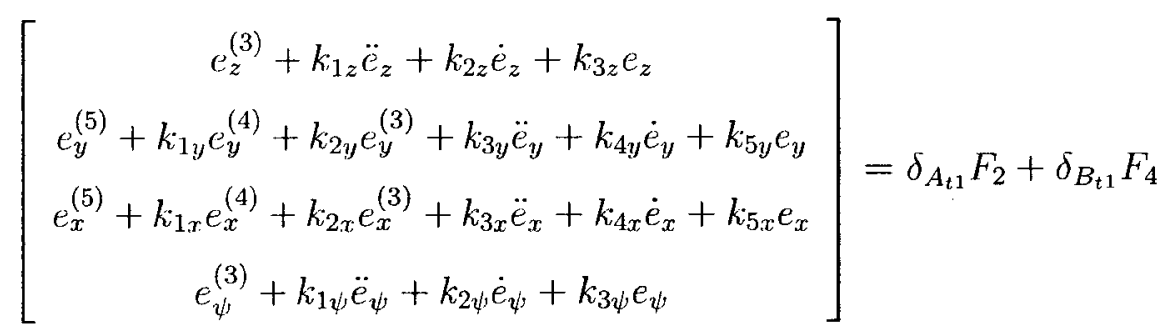

It is possible to represent the above equation in the state-space model. The selected state vector for this purpose is as follows

$$
X=\left[\begin{array}{llllllllllllllll}
e_{y} & e_{x} & \dot{e}_{y} & \dot{e}_{x} & e_{z} & \ddot{e}_{y} & \ddot{e}_{x} & e_{\psi} & \dot{e}_{z} & e_{y}^{(3)} & e_{x}^{(3)} & \dot{e}_{\psi} & \ddot{e}_{z} & e_{y}^{(4)} & e_{x}^{(4)} & \ddot{e}_{\psi}
\end{array}\right]^{T}
$$

Therefore, we could form the state-space representation according to the above 
state vector, that is

$$
\dot{X}=A_{16 \times 16} X+\delta_{A_{t 1}} F_{2}^{\prime}+\delta_{B_{t 1}} F_{4}^{\prime}
$$

where

$$
\begin{aligned}
F_{2}^{\prime} & =\left[\begin{array}{c}
0 \\
\vdots \\
0 \\
F_{2}
\end{array}\right]_{16 \times 1} \\
F_{4}^{\prime} & =\left[\begin{array}{c}
0 \\
\vdots \\
0 \\
F_{4}
\end{array}\right]_{16 \times 1}
\end{aligned}
$$

The $A_{(16 \times 16)}$ matrix in equation (4.40) is given by $\left[\begin{array}{cccccccccccccccc}0 & 0 & 1 & 0 & 0 & 0 & 0 & 0 & 0 & 0 & 0 & 0 & 0 & 0 & 0 & 0 \\ 0 & 0 & 0 & 1 & 0 & 0 & 0 & 0 & 0 & 0 & 0 & 0 & 0 & 0 & 0 & 0 \\ 0 & 0 & 0 & 0 & 0 & 1 & 0 & 0 & 0 & 0 & 0 & 0 & 0 & 0 & 0 & 0 \\ 0 & 0 & 0 & 0 & 0 & 0 & 1 & 0 & 0 & 0 & 0 & 0 & 0 & 0 & 0 & 0 \\ 0 & 0 & 0 & 0 & 0 & 0 & 0 & 0 & 1 & 0 & 0 & 0 & 0 & 0 & 0 & 0 \\ 0 & 0 & 0 & 0 & 0 & 0 & 0 & 0 & 0 & 1 & 0 & 0 & 0 & 0 & 0 & 0 \\ 0 & 0 & 0 & 0 & 0 & 0 & 0 & 0 & 0 & 0 & 1 & 0 & 0 & 0 & 0 & 0 \\ 0 & 0 & 0 & 0 & 0 & 0 & 0 & 0 & 0 & 0 & 0 & 1 & 0 & 0 & 0 & 0 \\ 0 & 0 & 0 & 0 & 0 & 0 & 0 & 0 & 0 & 0 & 0 & 0 & 1 & 0 & 0 & 0 \\ 0 & 0 & 0 & 0 & 0 & 0 & 0 & 0 & 0 & 0 & 0 & 0 & 0 & 1 & 0 & 0 \\ 0 & 0 & 0 & 0 & 0 & 0 & 0 & 0 & 0 & 0 & 0 & 0 & 0 & 0 & 1 & 0 \\ 0 & 0 & 0 & 0 & 0 & 0 & 0 & 0 & 0 & 0 & 0 & 0 & 0 & 0 & 0 & 1 \\ 0 & 0 & 0 & 0 & -k_{3 z} & 0 & 0 & 0 & -k_{2 z} & 0 & 0 & 0 & -k_{1 z} & 0 & 0 & 0 \\ -k_{5 y} & 0 & -k_{4 y} & 0 & 0 & -k_{3 y} & 0 & 0 & 0 & -k_{2 y} & 0 & 0 & 0 & -k_{1 y} & 0 & 0 \\ 0 & -k_{5 x} & 0 & -k_{4 x} & 0 & 0 & -k_{3 x} & 0 & 0 & 0 & -k_{2 x} & 0 & 0 & 0 & -k_{1 x} & 0 \\ 0 & 0 & 0 & 0 & 0 & 0 & 0 & -k_{3 \psi} & 0 & 0 & 0 & -k_{2 \psi} & 0 & 0 & 0 & -k_{1 \psi}\end{array}\right]$

All the eigenvalues of this matrix are negative according to the selection of the $k_{i}$ parameters, in other words, $A$ is a Hurwitz matrix. 
Now we define the update law for the estimation errors $\delta_{A_{t 1}}$ and $\delta_{B_{t 1}}$ according to the following equation

$$
\left\{\begin{array}{l}
\dot{\delta}_{A_{t 1}}=-\left(F_{2}^{\prime T} P X+X^{T} P F_{2}^{\prime}\right) \\
\dot{\delta}_{B_{t 1}}=-\left(F_{4}^{\prime T} P X+X^{T} P F_{4}^{\prime}\right)
\end{array}\right.
$$

where $X$ is the state vector defined in equation (4.39), and $P$ is a $(16 \times 16)$ matrix obtained by solving the following Lyapunov equation, that is

$$
A^{T} P+P A=-I_{16 \times 16}
$$

where $A$ matrix is defined in (4.42) and the notation $I_{16 \times 16}$ denotes an identity matrix with dimension $16 \times 16$. It should be noted that since $A$ is a Hurwitz matrix, $P$ is a positive definite matrix $[65]$.

The following theorem provides a sufficient condition for stability of the closedloop system.

Theorem 2. The state trajectories of system (4.40) with the update law for the estimation errors $\delta_{A_{t 1}}$ and $\delta_{B_{t 1}}$ given in equation (4.43) are globally stable in the sense of Lyapunov.

Proof: To carry out the stability analysis, choose the Lyapanov function candidate as

$$
V=\left(X^{T} P X+\frac{1}{2} \delta_{A_{t 1}}^{2}+\frac{1}{2} \delta_{B_{t 1}}^{2}\right)
$$

The derivative of this Lyapunov function along the state trajectories of system (4.40) yields

$$
\dot{V}=\dot{X}^{T} P X+X^{T} P \dot{X}+\delta_{A_{t 1}} \dot{\delta}_{A_{t 1}}+\delta_{B_{t 1}} \dot{\delta}_{B_{t 1}}
$$


Now, using (4.43) one arrives at the following relation

$$
\begin{aligned}
\dot{V} & =\left(A X+\delta_{A_{t 1}} F_{2}^{\prime}+\delta_{B_{t 1}} F_{4}^{\prime}\right)^{T} P X+X^{T} P\left(A X+\delta_{A_{t 1}} F_{2}^{\prime}+\delta_{B_{t 1}} F_{4}^{\prime}\right) \\
& +\delta_{A_{t 1}}\left(-F_{2}^{\prime T} P X-X^{T} P F_{2}^{\prime}\right)+\delta_{B_{t 1}}\left(-F_{4}^{\prime T} P X-X^{T} P F_{4}^{\prime}\right) \\
& =X^{T} A^{T} P X+X^{T} P A X \\
& =X^{T}\left(A^{T} P+P A\right) X
\end{aligned}
$$

It can be concluded from (4.44) that

$$
\dot{V}=-X^{T} X
$$

which guarantees the negative semi-definiteness of the derivative function $\dot{V}$. This implies that the origin is a globally stable equilibrium point of the system (4.40).

Remark 1. Stability of system (4.40) implies that the state variables defined in (4.39) as well as the estimation errors remain bounded as $t \longrightarrow \infty$.

In this section, we studied the case of partial LOE fault recovery by assuming that the fault has occurred in the first actuator. The same method could be applied to design three other controllers that accommodate the LOE fault in the other three actuators. In other words, this module of the fault recovery contains four different controllers and based upon the information from the FDI unit the proper controller is selected. In each of these controllers, two parameters, $A_{t i}$ and $B_{t i}$, related to the faulty $i^{\text {th }}$ actuator are assumed to be unknown and the parameters related to the healthy actuators are assumed to be known.

It is possible to extend this adaptive FL controller so that only one controller is designed that is capable of accommodating the partial LOE fault in all actuators. For this purpose, we assume that $A_{t i}$ and $B_{t i}$ for $i=1, \ldots, 4$ are unknown parameters and should be estimated. Therefore, in case of multiple LOE faults in two or more actuators, this controller is capable to provide estimates of the parameters that are 
subject to change and guarantee the system stability. This is done in the next section.

\subsubsection{Generalization of the Adaptive Feedback Lineariza- tion}

In this section, we assume that the thrust dynamic parameters according to all the actuators are unknown variables to be estimated and may have changed due to partial LOE faults. Therefore,

$$
\dot{T}_{i}=A_{t} T_{i}+B_{t} u_{i}+C_{t} \quad \text { for } \quad \mathrm{i}=1, \ldots, 4
$$

where $A_{t i}=k_{i}^{2} A_{t}$ and $B_{t i}=k_{i}^{2} B_{t}$ for $i=1, \ldots, 4$.

Similar to the previous section, it is possible to rewrite the movement vector dynamic equation (4.15) to separate the unknown parameters, namely

$$
\begin{aligned}
& {\left[\begin{array}{c}
\dot{U}_{1} \\
\dot{U}_{2} \\
\dot{U}_{3} \\
\dot{U}_{4}
\end{array}\right]=A_{t 1}\left[\begin{array}{c}
\frac{1}{4} U_{1}-\frac{1}{2} U_{3}-\frac{b}{4 d} U_{4} \\
0 \\
-\frac{l}{4} U_{1}+\frac{1}{2} U_{3}+\frac{l b}{4 d} U_{4} \\
-\frac{d}{4 b} U_{1}+\frac{d}{2 l b} U_{3}+\frac{1}{4} U_{4}
\end{array}\right]+A_{t 2}\left[\begin{array}{c}
\frac{1}{4} U_{1}-\frac{1}{2 l} U_{2}+\frac{b}{4 d} U_{4} \\
-\frac{l}{4} U_{1}+\frac{1}{2} U_{2}-\frac{l b}{4 d} U_{4} \\
0 \\
\frac{d}{4 b} U_{1}-\frac{d}{2 l b} U_{2}+\frac{1}{4} U_{4}
\end{array}\right]} \\
& +A_{t 3}\left[\begin{array}{c}
\frac{1}{4} U_{1}+\frac{1}{2} U_{3}-\frac{b}{4 d} U_{4} \\
0 \\
\frac{l}{4} U_{1}+\frac{1}{2} U_{3}-\frac{l b}{4 d} U_{4} \\
-\frac{d}{4 b} U_{1}-\frac{d}{2 l b} U_{3}+\frac{1}{4} U_{4}
\end{array}\right]+A_{t 4}\left[\begin{array}{c}
\frac{1}{4} U_{1}+\frac{1}{2 l} U_{2}+\frac{b}{4 d} U_{4} \\
\frac{l}{4} U_{1}+\frac{1}{2} U_{2}+\frac{l b}{4 d} U_{4} \\
0 \\
\frac{d}{4 b} U_{1}+\frac{d}{2 l b} U_{2}+\frac{1}{4} U_{4}
\end{array}\right] \\
& +\left[\begin{array}{cccc}
B_{t 1} & B_{t 2} & B_{t 3} & B_{t 4} \\
0 & -l B_{t 2} & 0 & B_{t 4} \\
-l B_{t 1} & 0 & l B_{t 3} & 0 \\
-\frac{d}{b} B_{t 1} & \frac{d}{b} B_{l 2} & -\frac{d}{b} B_{t 3} & \frac{d}{b} B_{t 4}
\end{array}\right]\left[\begin{array}{l}
u_{1} \\
u_{2} \\
u_{3} \\
u_{4}
\end{array}\right]+\left(L_{U T} C_{T}\right)
\end{aligned}
$$


The above dynamic equation is then substituted in the equations (4.20) to (4.23) and can be rewritten in a matrix form, that is

$$
\left[\begin{array}{l}
z^{(3)} \\
y^{(5)} \\
x^{(5)} \\
\psi^{(3)}
\end{array}\right]=H_{0}+A_{t 1} H_{1}+A_{t 2} H_{2}+A_{t 3} H_{3}+A_{t 4} H_{4}+H_{5}\left[\begin{array}{cccc}
B_{t 1} & 0 & 0 & 0 \\
0 & B_{t 2} & 0 & 0 \\
0 & 0 & B_{t 3} & 0 \\
0 & 0 & 0 & B_{t 4}
\end{array}\right]\left[\begin{array}{c}
u_{1} \\
u_{2} \\
u_{3} \\
u_{4}
\end{array}\right]
$$

where

$$
\begin{gathered}
H_{0}=\left[\begin{array}{c}
-\dot{\phi} \sin \phi \cos \theta \frac{U_{1}}{m}-\dot{\theta} \cos \phi \sin \theta \frac{U_{1}}{m}+4 C_{t} \\
3 g \frac{U_{2}}{I_{x x}} \dot{\phi} \sin \phi+g \dot{\phi}^{3} \cos \phi \\
-3 g \frac{U_{3}}{I_{y y}} \dot{\theta} \sin \theta-g \dot{\theta}^{3} \cos \theta \\
0
\end{array}\right] \\
H_{1}=\left[\begin{array}{c}
\frac{\cos \phi \cos \theta}{m}\left(\frac{1}{4} U_{1}-\frac{1}{2} U_{3}-\frac{b}{4 d} U_{4}\right) \\
0 \\
\frac{g \cos \theta}{I_{y y}}\left(-\frac{l}{4} U_{1}+\frac{1}{2} U_{3}+\frac{l b}{4 d} U_{4}\right) \\
\frac{1}{I_{z z}}\left(-\frac{d}{4 b} U_{1}+\frac{d}{2 l b} U_{3}+\frac{1}{4} U_{4}\right)
\end{array}\right] \\
H_{2}=\left[\begin{array}{c}
\frac{\cos \phi \cos \theta}{m}\left(\frac{1}{4} U_{1}-\frac{1}{2 l} U_{2}+\frac{b}{4 d} U_{4}\right) \\
\frac{-g \cos \phi}{I_{x x}}\left(-\frac{l}{4} U_{1}+\frac{1}{2} U_{2}-\frac{l b}{4 d} U_{4}\right) \\
0 \\
0 \\
\frac{1}{I_{z z}}\left(\frac{d}{4 b} U_{1}-\frac{d}{2 l b} U_{2}+\frac{1}{4} U_{4}\right) \\
\frac{\cos \phi \cos \theta}{m}\left(\frac{1}{4} U_{1}+\frac{1}{2} U_{3}-\frac{b}{4 d} U_{4}\right) \\
\frac{g \cos \theta}{I_{y y}}\left(\frac{l}{4} U_{1}+\frac{1}{2} U_{3}-\frac{l b}{4 d} U_{4}\right) \\
\frac{1}{I_{z z}}\left(-\frac{d}{2 b} U_{1}-\frac{d}{2 l b} U_{3}+\frac{1}{4} U_{4}\right)
\end{array}\right]
\end{gathered}
$$




$$
\begin{gathered}
H_{4}=\left[\begin{array}{c}
\frac{\cos \phi \cos \theta}{m}\left(\frac{1}{4} U_{1}+\frac{1}{2 l} U_{2}+\frac{b}{4 d} U_{4}\right) \\
\frac{-g \cos \phi}{I_{x x}}\left(\frac{l}{4} U_{1}+\frac{1}{2} U_{2}+\frac{l b}{4 d} U_{4}\right) \\
0 \\
\frac{1}{I_{z z}}\left(\frac{d}{4 b} U_{1}+\frac{d}{2 l b} U_{2}+\frac{1}{4} U_{4}\right)
\end{array}\right] \\
H_{5}=\left[\begin{array}{cccc}
\frac{\cos \phi \cos \theta}{m} & \frac{\cos \phi \cos \theta}{m} & \frac{\cos \phi \cos \theta}{m} & \frac{\cos \phi \cos \theta}{m} \\
0 & \frac{g \cos \phi}{I_{x x}} l & 0 & -\frac{g \cos \phi}{I_{x x}} l \\
-\frac{g \cos \theta}{I_{y y}} l & 0 & \frac{g \cos \theta}{I_{y y}} l & 0 \\
\frac{1}{I_{z z}}\left(-\frac{d}{b}\right) & \frac{1}{I_{z z}}\left(\frac{d}{b}\right) & \frac{1}{I_{z z}}\left(-\frac{d}{b}\right) & \frac{1}{I_{z z}}\left(\frac{d}{b}\right)
\end{array}\right]
\end{gathered}
$$

The input signal $u$ is defined in (4.53) so that the system becomes linear, namely

$$
\left[\begin{array}{l}
u_{1} \\
u_{2} \\
u_{3} \\
u_{4}
\end{array}\right]=\left(H_{5}\left[\begin{array}{cccc}
\hat{B}_{\ell 1} & 0 & 0 & 0 \\
0 & \hat{B}_{t 2} & 0 & 0 \\
0 & 0 & \hat{B}_{t 3} & 0 \\
0 & 0 & 0 & \hat{B}_{t 4}
\end{array}\right]\right)^{-1}\left(\left[\begin{array}{c}
w_{1} \\
w_{2} \\
w_{3} \\
w_{4}
\end{array}\right]-H_{0}-\hat{A}_{t 1} H_{1}-\hat{A}_{t 2} H_{2}-\hat{A}_{t 3} H_{3}-\hat{A}_{t 4} H_{4}\right)
$$

where $\hat{A}_{t i}$ and $\hat{B}_{t i}$ are the estimates of the unknown parameters $A_{t i}$ and $B_{t i}$ for $i=1, \ldots, 4$.

Similar to the previous section, it is possible to form the closed-loop equation of the feedback linearized system as follows

$$
\left[\begin{array}{l}
z^{(3)} \\
y^{(5)} \\
x^{(5)} \\
\psi^{(3)}
\end{array}\right]=\left[\begin{array}{l}
w_{1} \\
w_{2} \\
w_{3} \\
w_{4}
\end{array}\right]+\sum_{i=1}^{4}\left(\delta_{A_{t i}} H_{i}\right)+\sum_{i=1}^{4}\left(\delta_{B_{t i}} G_{i}\right)
$$


where

$$
\begin{aligned}
& G_{1}=H_{5}\left[\begin{array}{cccc}
\frac{1}{\hat{B}_{t 1}} & 0 & 0 & 0 \\
0 & 0 & 0 & 0 \\
0 & 0 & 0 & 0 \\
0 & 0 & 0 & 0
\end{array}\right] H_{5}^{-1}\left(\left[\begin{array}{c}
w_{1} \\
w_{2} \\
w_{3} \\
w_{4}
\end{array}\right]-H_{0}-\hat{A}_{t 1} H_{1}-\hat{A}_{t 2} H_{2}-\hat{A}_{t 3} H_{3}-\hat{A}_{t 4} H_{4}\right) \\
& G_{2}=H_{5}\left[\begin{array}{cccc}
0 & 0 & 0 & 0 \\
0 & \frac{1}{\hat{B}_{t 2}} & 0 & 0 \\
0 & 0 & 0 & 0 \\
0 & 0 & 0 & 0
\end{array}\right] H_{5}^{-1}\left(\left[\begin{array}{c}
w_{1} \\
w_{2} \\
w_{3} \\
w_{4}
\end{array}\right]-H_{0}-\hat{A}_{t 1} H_{1}-\hat{A}_{t 2} H_{2}-\hat{A}_{t 3} H_{3}-\hat{A}_{t 4} H_{4}\right) \\
& G_{3}=H_{5}\left[\begin{array}{cccc}
0 & 0 & 0 & 0 \\
0 & 0 & 0 & 0 \\
0 & 0 & \frac{1}{\hat{B}_{t 3}} & 0 \\
0 & 0 & 0 & 0
\end{array}\right] H_{5}^{-1}\left(\left[\begin{array}{c}
w_{1} \\
w_{2} \\
w_{3} \\
w_{4}
\end{array}\right]-H_{0}-\hat{A}_{t 1} H_{1}-\hat{A}_{t 2} H_{2}-\hat{A}_{t 3} H_{3}-\hat{A}_{t 4} H_{4}\right) \\
& G_{4}=H_{5}\left[\begin{array}{cccc}
0 & 0 & 0 & 0 \\
0 & 0 & 0 & 0 \\
0 & 0 & 0 & 0 \\
0 & 0 & 0 & \frac{1}{\hat{B}_{t 3}}
\end{array}\right] H_{5}^{-1}\left(\left[\begin{array}{c}
w_{1} \\
w_{2} \\
w_{3} \\
w_{4}
\end{array}\right]-H_{0}-\hat{A}_{t 1} H_{1}-\hat{A}_{t 2} H_{2}-\hat{A}_{t 3} H_{3}-\hat{A}_{t 4} H_{4}\right)
\end{aligned}
$$

The control inputs $w_{1}, w_{2}, w_{3}$ and $w_{4}$ are defined according to the equations (4.28) to $(4.31)$.

The update law for the parameter estimation error signals $\delta_{A_{t i}}$ and $\delta_{B_{t i}}$ is then set as

$$
\left\{\begin{array}{l}
\dot{\delta}_{A_{t i}}=-\left(H_{i}^{\prime T} P X+X^{T} P H_{i}^{\prime}\right) \\
\dot{\delta}_{B_{t i}}=-\left(G_{i}^{\prime T} P X+X^{T} P G_{i}^{\prime}\right)
\end{array}\right.
$$

where the matrix $P$ and the vector $X$ are given by (4.39) and (4.44), and the vectors 
$H_{i}^{\prime}$ and $G_{i}^{\prime}$ are defined as follows

$$
\begin{aligned}
H_{i}^{\prime} & =\left[\begin{array}{c}
0 \\
\vdots \\
0 \\
H_{i}
\end{array}\right]_{16 \times 1} \\
G_{i}^{\prime} & =\left[\begin{array}{c}
0 \\
\vdots \\
0 \\
G_{i}
\end{array}\right]_{16 \times 1}
\end{aligned}
$$

The following theorem is an generalization to Theorem 2 and provides a sufficient condition for the stability of the closed-loop system (4.54) and (4.56).

Theorem 3. The state trajectories of system (4.54) with the update law for the estimation errors $\delta_{A_{t i}}$ and $\delta_{B_{t i}}$ for $i=1, \ldots 4$ given in equation (4.56) are globally stable in the sense of Lyapunov.

Proof: To carry out the stability analysis, choose the Lyapanov function candidate as

$$
V=\left(X^{T} P X+\frac{1}{2} \sum_{i=1}^{4} \delta_{A_{t i}}^{2}+\frac{1}{2} \sum_{i=1}^{4} \delta_{B_{t i}}^{2}\right)
$$

The derivative of this Lyapunov function along the state trajectories of the system in (4.54) yields

$$
\dot{V}=\dot{X}^{T} P X+X^{T} P \dot{X}+\sum_{i=1}^{4} \delta_{A_{t i}} \dot{\delta}_{A_{t i}}+\sum_{i=1}^{4} \delta_{B_{t i}} \dot{\delta}_{B_{t i}}
$$

Now, using (4.56) one arrives at the following relation

$$
\dot{V}=-X^{T} X
$$


which guarantees the negative semi-definiteness of $\dot{V}$. This implies that the origin is a globally stable equilibrium point of the system (4.54) and (4.56).

Remark 2. Stability of the system (4.54) and (4.56) implies that the state variables defined in (4.39) as well as the estimation errors remain bounded as $t \longrightarrow \infty$.

The above theorem states that the control of the system is no longer dependent on prior knowledge of the actuator thrust dynamic parameters and these parameters could be estimated from the input and states signals of the system, provided that all the states of the system are available. Therefore, if the LOE fault occur in one of the actuators, the parameter estimation module is able to estimate the post fault model of the actuators and compensates for the error effects in the system by proper commanding the healthy and faulty actuators and guarantee the closed-loop stability of the system.

\subsection{LIP, Float and HOF Faults Modeling}

In this section, a short discussion on the effects of LIP, float and HOF faults in an actuator is provided. However, our proposed method for adaptive feedback linearization is shown to be inadequate for these types of faults as a result of the fact that the quadrotor is an under-actuated system.

In case of these types of faults, one of the actuators is either stuck in a certain speed with no possibility of controlling it as in the LIP and HOF or fully nonfunctional as in the float fault. Without loss of generality, let us assume that one of these faults has occurred in the first rotor and the remaining actuators are healthy. Therefore, the speed of the first rotor has been frozen at a certain point, that is

$$
\Omega_{1}=\bar{\Omega}_{1}
$$


where

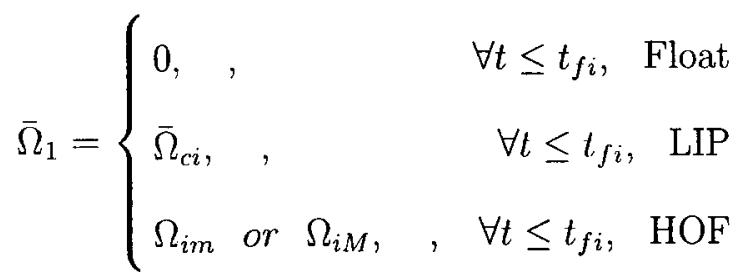

where $\bar{\Omega}_{1}$ is the actual output of the actuator, $\bar{\Omega}_{c i}$ is a value within the range of operation of the $i^{t h}$ actuator frozen at the time of failure and $\Omega_{i m}$ and $\Omega_{i M}$ are the upper and lower limit.

The resulting thrust force from the faulty first actuator is given by

$$
T_{1}=b \bar{\Omega}_{1}^{2}
$$

The term $\dot{T}_{1}$ becomes zero as $T_{1}$ stays constant, that is

$$
\left\{\begin{array}{l}
\dot{T}_{1}=0 \\
\dot{T}_{i}=A_{t} T_{i}+B_{t} u_{i}+C_{t} \quad \text { for } \quad \mathrm{i}=2,3,4
\end{array}\right.
$$

The input moments after the total LOE fault would also change according to the following equation, namely

$$
\left\{\begin{array}{l}
U_{1}=b \bar{\Omega}_{1}^{2}+T_{2}+T_{3}+T_{4} \\
U_{2}=-l T_{2}+l T_{4} \\
U_{3}=-l\left(b \bar{\Omega}_{1}^{2}\right)+l T_{3} \\
U_{4}=-\frac{d}{b}\left(b \bar{\Omega}_{1}^{2}\right)+\frac{d}{b} T_{2}-\frac{d}{b} T_{3}+\frac{d}{b} T_{4}
\end{array}\right.
$$

Therefore, the dynamics of the input moments become 


$$
\left\{\begin{array}{l}
\dot{U}_{1}=A_{t}\left(T_{2}+T_{3}+T_{4}\right)+B_{t}\left(u_{2}+u_{3}+u_{4}\right)+4 C_{t} \\
\dot{U}_{2}=l A_{t}\left(-T_{2}+T_{4}\right)+l B_{t}\left(-u_{2}+u_{4}\right) \\
\dot{U}_{3}=l A_{t} T_{3}+l B_{t} u_{3}+C_{t} \\
\dot{U}_{4}=\frac{d}{b} A_{t}\left(T_{2}-T_{3}+T_{4}\right)+\frac{d}{b} B_{t}\left(u_{2}-u_{3}+u_{4}\right)
\end{array}\right.
$$

It is possible to rewrite the dynamic equations in (4.66) in a matrix form as in the following

$$
\left[\begin{array}{c}
\dot{U}_{1} \\
\dot{U}_{2} \\
\dot{U}_{3} \\
\dot{U}_{4}
\end{array}\right]=A_{t}\left[\begin{array}{c}
U_{1} \\
U_{2} \\
U_{3} \\
U_{4}
\end{array}\right]+B_{t}\left[\begin{array}{ccc}
1 & 1 & 1 \\
-l & 0 & l \\
0 & l & 0 \\
\frac{d}{b} & -\frac{d}{b} & \frac{d}{b}
\end{array}\right]\left[\begin{array}{c}
u_{2} \\
u_{3} \\
u_{4}
\end{array}\right]+\left[\begin{array}{c}
-A_{t}\left(b \bar{\Omega}_{1}^{2}\right)+4 C_{\iota} \\
0 \\
A_{t} l\left(b \bar{\Omega}_{1}^{2}\right)+C_{t} \\
\frac{d}{b} A_{t}\left(b \bar{\Omega}_{1}^{2}\right)
\end{array}\right]
$$

Similar to the previous sections, the goal is to control the position $(x, y, z)$ and the heading angle, $\psi$, of the quadrotor. Substituting the above dynamic equation in equations $(4.20)$ to $(4.23)$ results in the following open-loop dynamic equations for the quadrotor system, namely

$$
\left[\begin{array}{c}
z^{(3)} \\
y^{(5)} \\
x^{(5)} \\
\psi^{(3)}
\end{array}\right]=G_{1}+G_{2}\left(A_{t}\left[\begin{array}{c}
U_{1} \\
U_{2} \\
U_{3} \\
U_{4}
\end{array}\right]+B_{t}\left[\begin{array}{ccc}
1 & 1 & 1 \\
-l & 0 & l \\
0 & l & 0 \\
\frac{d}{b} & -\frac{d}{b} & \frac{d}{b}
\end{array}\right]\left[\begin{array}{c}
u_{2} \\
u_{3} \\
u_{4}
\end{array}\right]+\left[\begin{array}{c}
-A_{t}\left(b \bar{\Omega}_{1}^{2}\right)+4 C_{t} \\
0 \\
A_{t} l\left(b \bar{\Omega}_{1}^{2}\right)+C_{t} \\
\frac{d}{b} A_{t}\left(b \bar{\Omega}_{1}^{2}\right)
\end{array}\right]\right)
$$

where

$$
G_{1}=\left[\begin{array}{c}
-\dot{\phi} \sin \phi \cos \theta \frac{U_{1}}{m}-\dot{\theta} \cos \phi \sin \theta \frac{U_{1}}{m} \\
3 g \frac{U_{2}}{I_{x x}} \dot{\phi} \sin \phi+g \dot{\phi}^{3} \cos \phi \\
-3 g \frac{U_{3}}{I_{y y}} \dot{\theta} \sin \theta-g \dot{\theta}^{3} \cos \theta \\
0
\end{array}\right]
$$




$$
G_{2}=\left[\begin{array}{cccc}
\frac{\cos \phi \cos \theta}{m} & 0 & 0 & 0 \\
0 & \frac{-g \cos \phi}{I_{x x}} & 0 & 0 \\
0 & 0 & \frac{g \cos \theta}{I_{y y}} & 0 \\
0 & 0 & 0 & \frac{1}{I_{z z}}
\end{array}\right]
$$

We are seeking a control law to first feedback linearize the system equations and then to stabilize the closed-loop dynamics. If we let the linearized equation to be

$$
\left[\begin{array}{l}
z^{(3)} \\
y^{(5)} \\
x^{(5)} \\
\psi^{(3)}
\end{array}\right]=\left[\begin{array}{l}
w_{1} \\
w_{2} \\
w_{3} \\
w_{4}
\end{array}\right]
$$

then the input voltage to the remaining healthy actuators should satisfy the following equation, that is

$$
\left[\begin{array}{ccc}
1 & 1 & 1 \\
-l & 0 & l \\
0 & l & 0 \\
\frac{d}{b} & -\frac{d}{b} & \frac{d}{b}
\end{array}\right]\left[\begin{array}{c}
u_{2} \\
u_{3} \\
u_{4}
\end{array}\right]=\frac{1}{B_{t}} G_{2}^{-1}\left(\left[\begin{array}{c}
w_{1} \\
w_{2} \\
w_{3} \\
w_{4}
\end{array}\right]-G_{1}-G_{2} A_{t}\left[\begin{array}{c}
U_{1} \\
U_{2} \\
U_{3} \\
U_{4}
\end{array}\right]-G_{2}\left[\begin{array}{c}
-A_{t}\left(b \bar{\Omega}_{1}^{2}\right)+4 C_{t} \\
0 \\
A_{t} l\left(b \bar{\Omega}_{1}^{2}\right)+C_{t} \\
\frac{d}{b} A_{t}\left(b \bar{\Omega}_{1}^{2}\right)
\end{array}\right]\right)
$$

It should be noted that the input vector $\left[\begin{array}{lll}u_{2} & u_{3} & u_{4}\end{array}\right]$ is left multiplied by a nonsquare matrix of the $(4 \times 3)$ dimension. Since the number of coloums is larger than the number of rows, this matrix is left invertible. In other words, since the number of free parameters $u_{i}$ for $i=2, \ldots, 4$ is less than the number of equations, which is four, the above equation does not have a solution.

If we select to only control three of the outputs, for example $z, y$ and $\psi$, the above equation has a unique solution as one of the equations would be deleted. The problem in this case is that by controlling only three outputs, the uncontrolled output could become unstable, for example $x$. The physical interpretation here 
could be explained by the fact that in hovering condition the thrust forces from all the actuators should satisfy the following criteria, that is

$$
\left\{\begin{array}{l}
T_{1}+T_{2}+T_{3}+T_{4}=U_{1}=m g \\
-T_{2}+T_{4}=0 \\
-T_{1}+T_{3}=0 \\
-T_{1}+T_{2}-T_{3}+T_{4}=0
\end{array}\right.
$$

where $m g$ is the force due to gravity. This means that at hovering $T_{i}=\frac{m g}{4}$ for $i=1, \ldots, 4$. Each of these equations is satisfied by controlling $z, y, x$ and $\psi$ respectively. Let's assume that a LIP, float or HOF fault has occurred in one of the actuators, i.e the first actuator, therefore, $T_{1}=b \bar{\Omega}_{1}^{2}$ and we select to control only three outputs say $z, y$ and $\psi$. We now have

$$
\left\{\begin{array}{l}
T_{2}+T_{3}+T_{4}=m g-b \bar{\Omega}_{1}^{2} \\
-T_{2}+T_{4}=0 \\
T_{2}-T_{3}+T_{4}=b \bar{\Omega}_{1}^{2}
\end{array}\right.
$$

Therefore,

$$
\left\{\begin{array}{l}
T_{1}=b \bar{\Omega}_{1}^{2} \\
T_{2}=\frac{m g}{2} \\
T_{3}=\frac{m g}{2}-b \bar{\Omega}_{1}^{2} \\
T_{4}=\frac{m g}{2}
\end{array}\right.
$$

This results in $-T_{1}+T_{3}=\frac{m g}{2}-2 k \neq 0$. This an additive force that generates a roll moment and results in an unstable movement, of the quadrotor in the $x$ direction. 


\subsection{Simulation Results}

In this section the behavior of the quadrotor model with the fault recovery mechanism is studied through simulation results. Similar to the previous chapter, the model used for simulation is OS4 developed in Ecole Polythechnique Federal De Lausanne [1]. The model used for control design is approximately nonlinear but we have implemented the fully nonlinear model of the quadrotor as well as the actuators for simulation and additive white Gaussian noise is also added to input and output channels to simulate a more realistic environment. The additive noise power is selected so that the signal to noise rate is approximately $15 \mathrm{db}$.

The desired trajectory commanded to the system is similar to the 1st trajectory in Chapter 3, Section 3.4. The mission starts at the position $(x, y, z)=(0,0,0)$ while the roll, the pitch and the yaw angles are initially equal to zero. The commanded trajectory is to fly from the initial point to the point $(10,10,10)(m)$ in 20 seconds and hovering at this point. The fault trajectory here assumes partial LOE failure in the first actuator at time $=50 \mathrm{sec}$. Three different fault severities are simulated where the first actuator losses $10 \%, 25 \%$ and $50 \%$ of its effectiveness. It should be noted that in this work we have not considered the limits on the input voltage to the actuators. Therefore, although it is possible to simulate higher severity of fault scenario, but this may not be practical due to physical limitations and constraints of the actuators in reality.

\section{$10 \%$ LOE in the 1st actuator}

The first set of simulations is related to a scenario where a $10 \%$ partial LOE fault has occured in the first actuator at time $=50 \mathrm{sec}$ without the fault recovery mechanism. Figures 4.2, 4.3, 4.4 and 4.5 show the linear position, Euler angles, input signals and the error signals corresponding to the commanded trajectory and the observed outputs for this scenario. It is seen that only $10 \%$ LOE in the first actuator leads to a relatively large error (approximately $80 \%$ ) in the output $x$. Higher severity 
of LOE fault in one actuator leads to significantly large error signals that even can make the system outputs unstable. Specifically, a $25 \%$ of LOE fault without fault recovery mechanism leads to an unstable system.

The second set of simulations is related to a scenario where the fault tolerant controller is implemented in the closed-loop system and a 10\% partial LOE fault has occured in the first actuator at time $=50 \mathrm{sec}$. Figures 4.6, 4.7 and 4.8 and 4.9 show the linear position, Euler angles, input signals and the estimated parameters of the faulty actuator corresponding to this scenario. Error signals are also depicted in Figure 4.10. It can be seen that with the parameter estimation module and the fault recovery algorithm, the outputs of the system affected by the LOE fault as depicted in the previous set of simulations, converge to the desired commanded trajectories even after the occurrence of the fault and the error signals also converge to zero as $t \longrightarrow \infty$.

\section{5\% LOE in the 1st Actutor}

Figures $4.11,4.12$ and 4.13 and 4.14 show the linear position, Euler angles, input signals and the estimated parameters of the faulty actuator corresponding to a $25 \%$ LOE fault in the first actuator at time $=50 \mathrm{sec}$. Error signals are also depicted in Figure 4.15. Without the fault recovery mechanism, the outputs of the system subject to $\% 25$ LOE fault become unstable but as can be seen with the fault recovery the error signals converge to zero after the fault occurence as $t \longrightarrow \infty$.

\section{$50 \%$ LOE in the 1st Actutor}

Figures $4.16,4.17$ and 4.18 and 4.19 show the linear position, Euler angles, input signals and the estimated parameters of the faulty actuator corresponding to a $50 \%$ LOE fault in the first actuator at time $=50 \mathrm{sec}$. Error signals are also depicted in Figure 4.20. Without the fault recovery mechanism, the outputs of the system subject to a $50 \%$ LOE fault become unstable but as can be seen with the fault recovery the error signals converge to zero after the fault occurence as $t \longrightarrow \infty$. 


\section{Multiple Faults in Two Actuators}

In order to show the performance of the adaptive feedback controller in case of multiple fault occurrences, we simulate the closed-loop system assuming that the $10 \%$ partial LOE fault will occur in both the first and the fourth actuators at different times, i.e. time $=50 \mathrm{sec}$ in the first one and time $=80 \mathrm{sec}$ in the fourth one. Figures 4.21, 4.22, 4.23 and 4.24 show the linear position, Euler angles, input signals and the error signals between the commanded trajectory and the observed outputs corresponding to this scenario, while there is no fault recovery mechanism. The effect of the LOE fault in the first actuator mostly appear on the $x$ output while the $y$ output is the one subject to change due to the fault in the fourth actuator. The error signals in this case show relatively considerable difference between the desired output and the observed ones.

Figures $4.25,4.26$ and 4.27 and 4.28 show the linear position, Euler angles, input signals and the estimated parameters of the faulty actuator corresponding to $10 \%$ LOE fault in the first actuator at time $=50 \mathrm{sec}$ and the same fault in the fourth actuator at time $=80 \mathrm{sec}$ with the fault recovery mechanism. The error signals are depicted in Figure 4.29 and as expected they converge to zero as $t \longrightarrow \infty$.

In order to gain a better understanding of the performance of the proposed adaptive feedback controller in presence of faults, Table 4.1 shows the mean and standard deviation values of the error signals in steady state for all the above scenarios. It should be noted that all the simulations were performed for the duration of 180 seconds and with the presence of the similar additive noise on the output signals as well as input signals. The results indicate, in general, that the system recovered from the LOE fault presents error signals with small mean values and prevents the faulty system to become unstable. In other words, the performance of

the system recovered from faults has considerably been improved with respect to the faulty system. 
Table 4.2 shows the mean values related to the input signals to the rotors in the above mentioned scenarios. It should be noted that we have not considered limits on the input signals in this work, however, in practice this is an important factor to be considered. Table 4.2 shows that due to higher LOE severity fault in an actuator, higher level of input voltage is required. It is possible to verify the maximum level of LOE fault that the quadrotor is capable of recovering by knowing a priori the limits and bounds on the input voltage. 


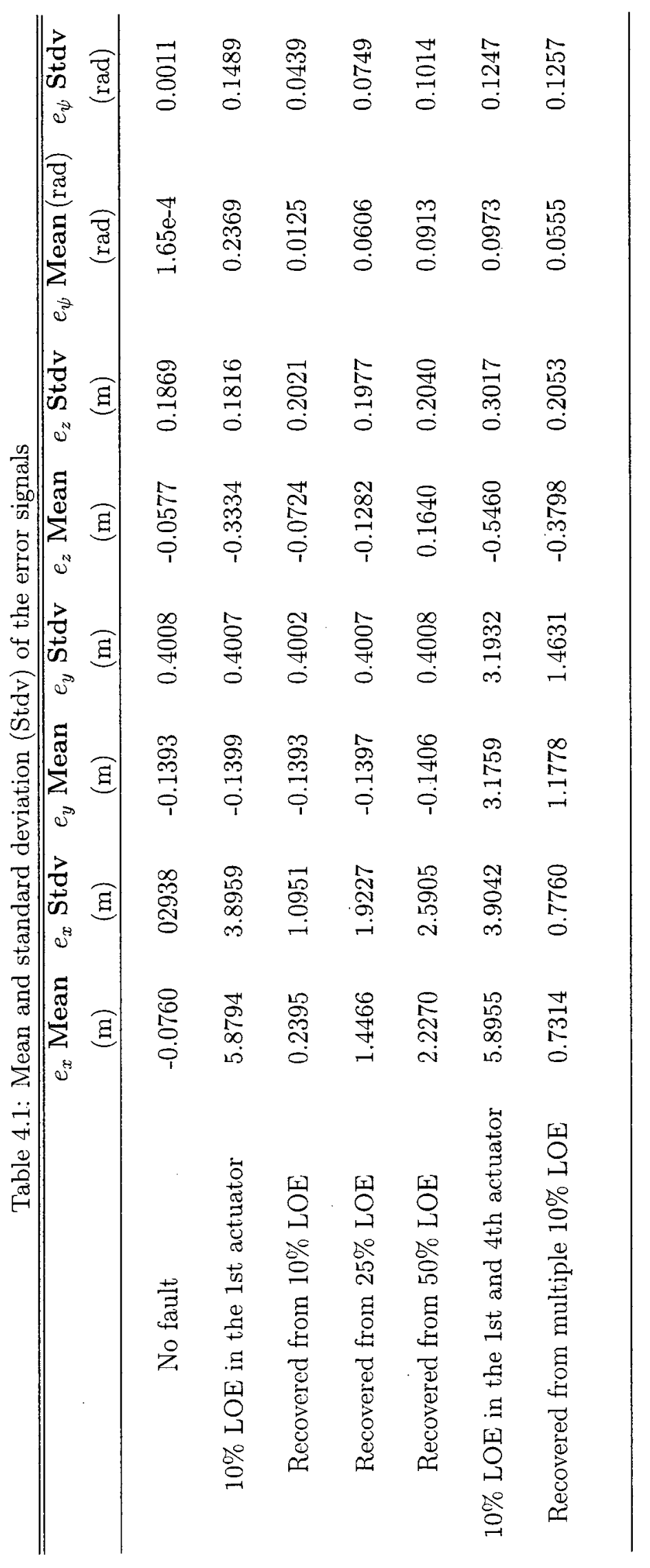




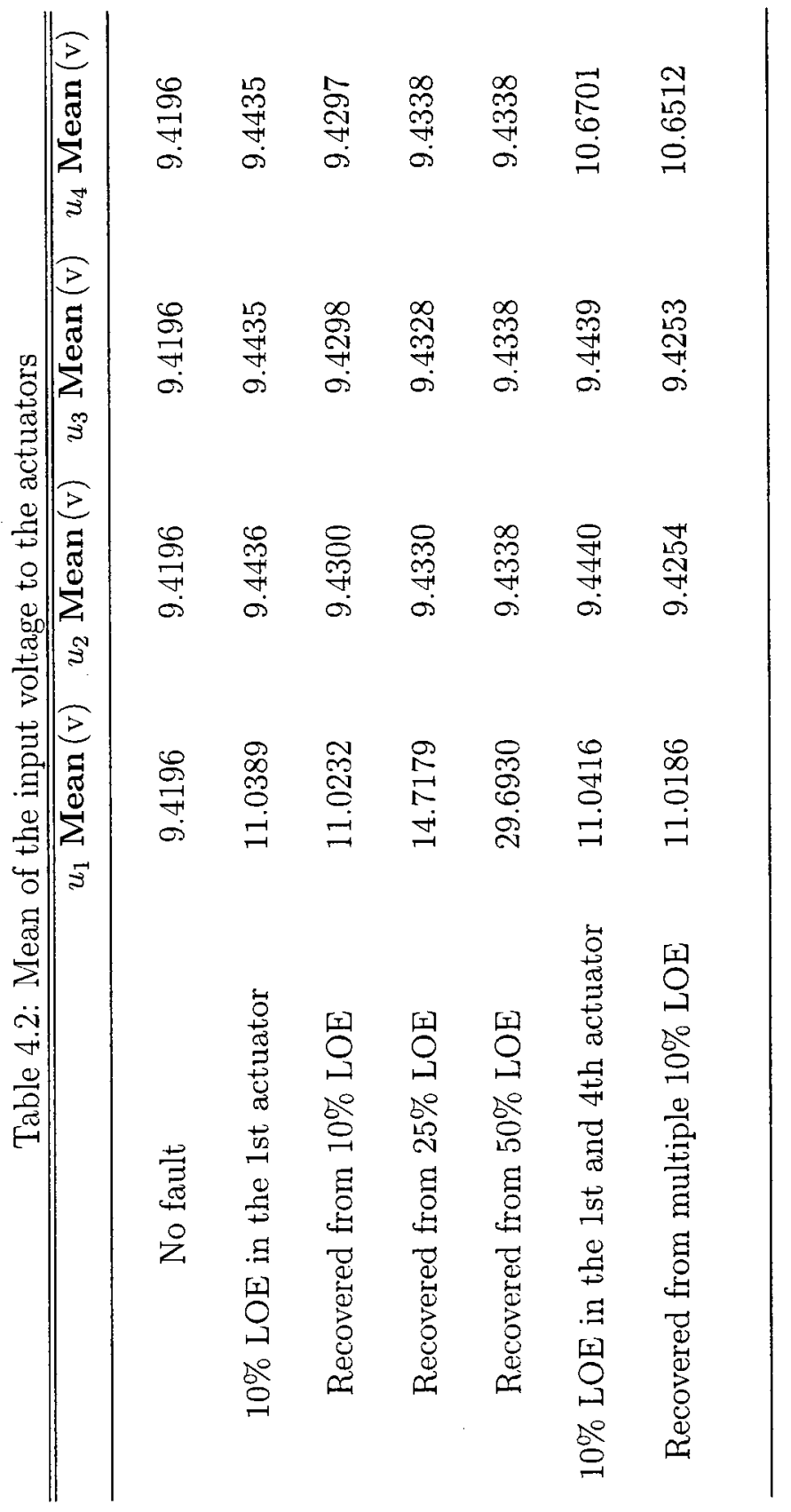




\subsection{Conclusions}

This chapter has presented different fault characteristics in actuators and the effects of actuator faults on the quadrotor system. The feedback linearization controller developed in Chapter 3 is generalized to be able to compensate partial LOE fault effects on the system. This is accomplished by introducing a parameter estimation algorithm and deriving proper update laws for the parameters subject to changes due to the presence of LOE fault. The algorithm is extended to monitor all the four actuators performance to be able to recover from multiple occurrence of LOE fault in different actuators. It is shown the system is able to recover from partial LOE fault automatically. Simulation results are presented in this chapter that show the effectiveness of our designed controller in presence of faults in actuators.

Next chapter will discuss the conclusions of this thesis and future work to be conducted on fault recovery of under-actuated quadrotor aerial vehicle. 
(a)

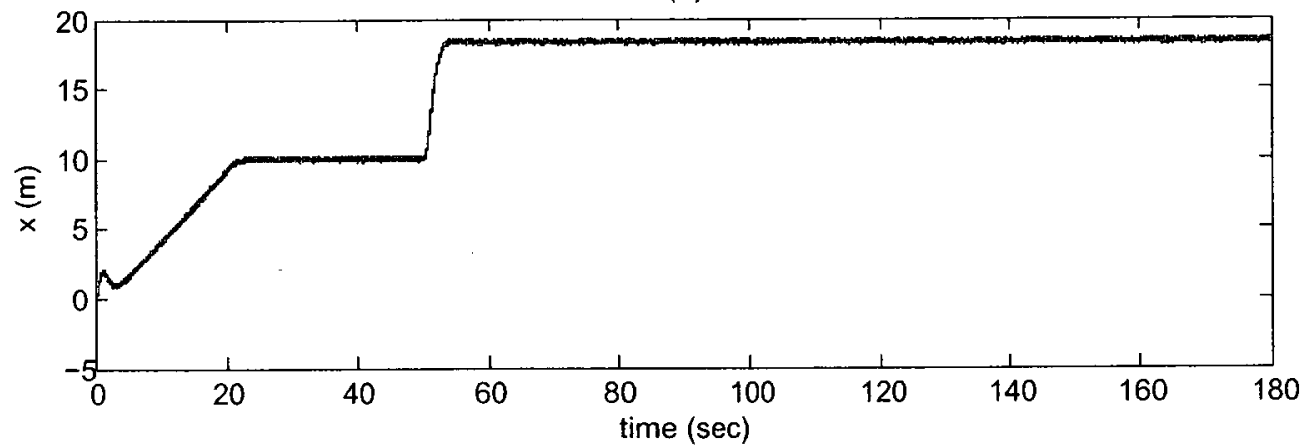

(b)

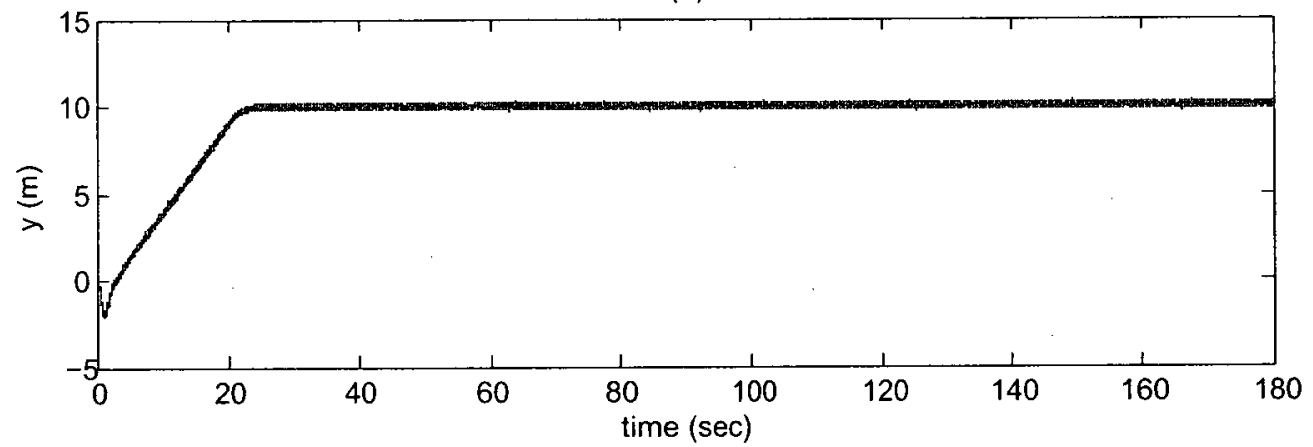

(c)

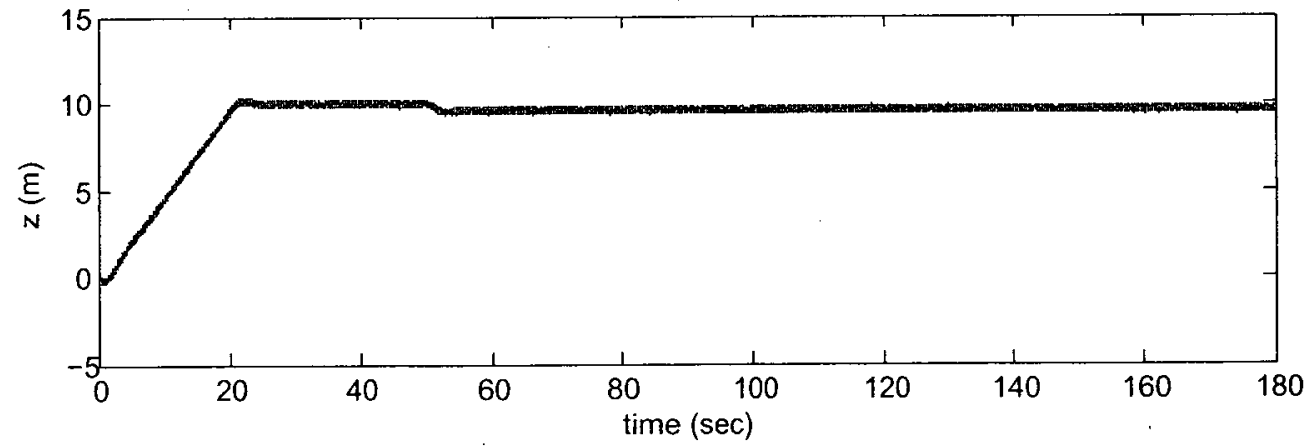

Figure 4.2: Linear position in response to the commanded trajectory corresponding to a $10 \%$ LOE fault in the first actuator and without fault recovery mechanism: (a) $\mathrm{x}$, (b) $\mathrm{y}$ and (c) $\mathrm{z}$, measured in meters. 
(a)

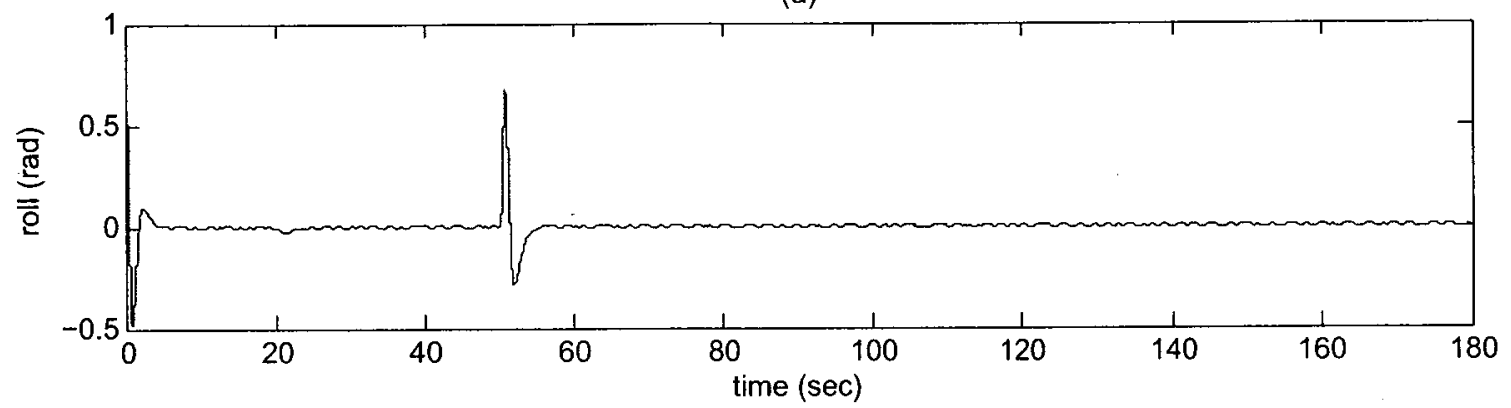

(b)

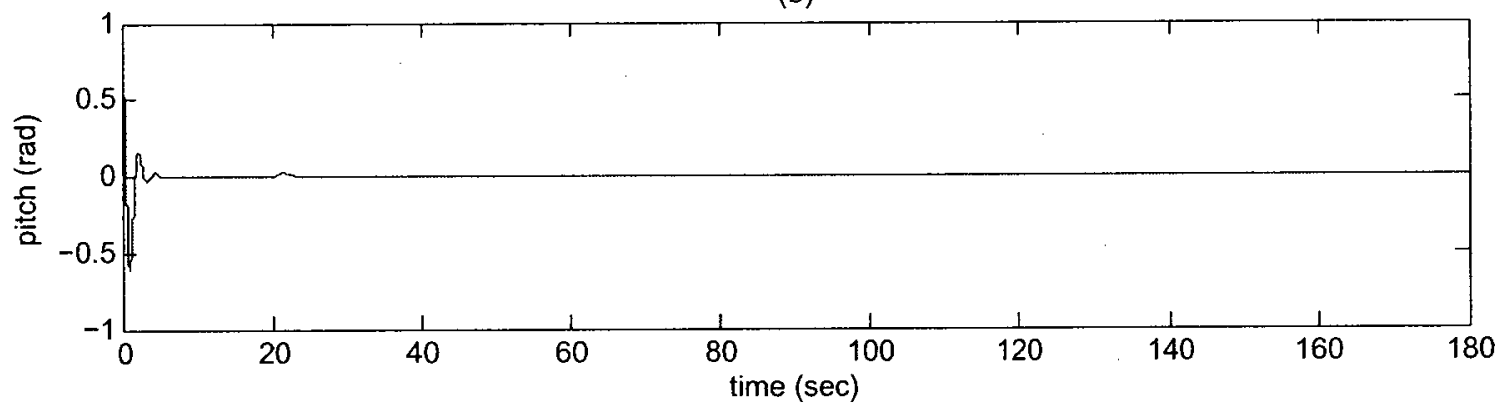

(c)

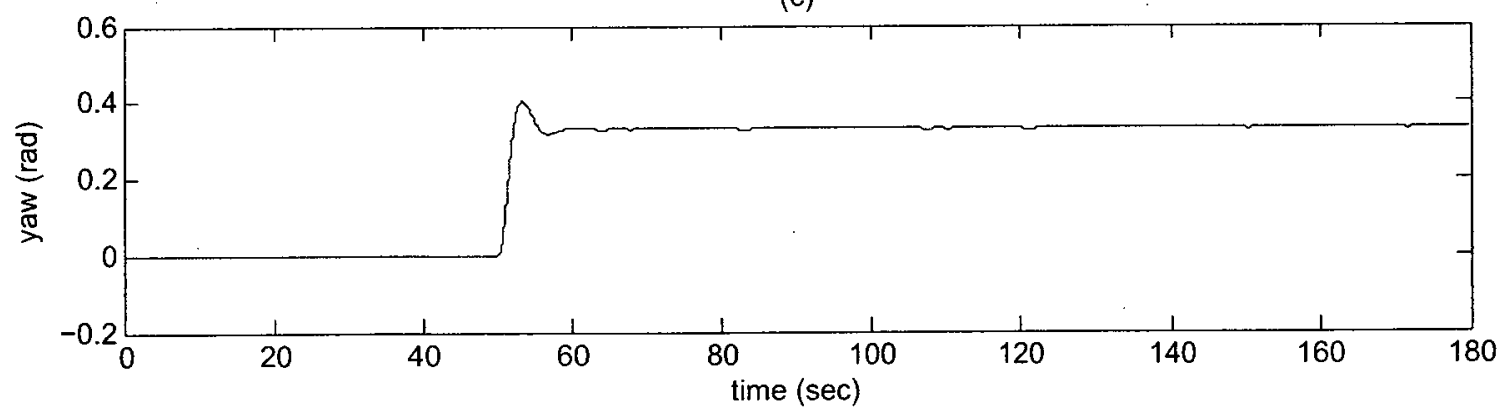

Figure 4.3: Euler angles in response to the commanded trajectory corresponding to a $10 \%$ LOE fault in the first actuator and without fault recovery mechanism: (a) Roll (rad), (b) Pitch (rad) and (c) Yaw (rad). 
(a)

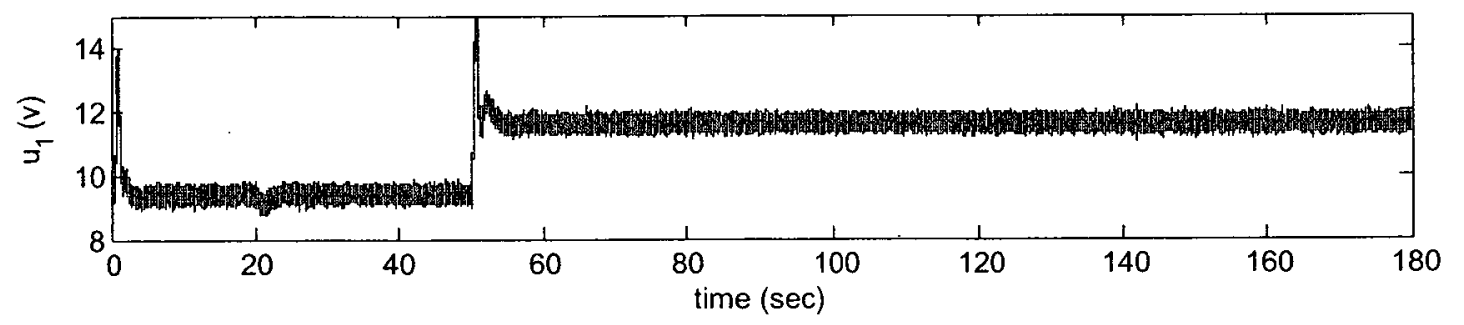

(b)

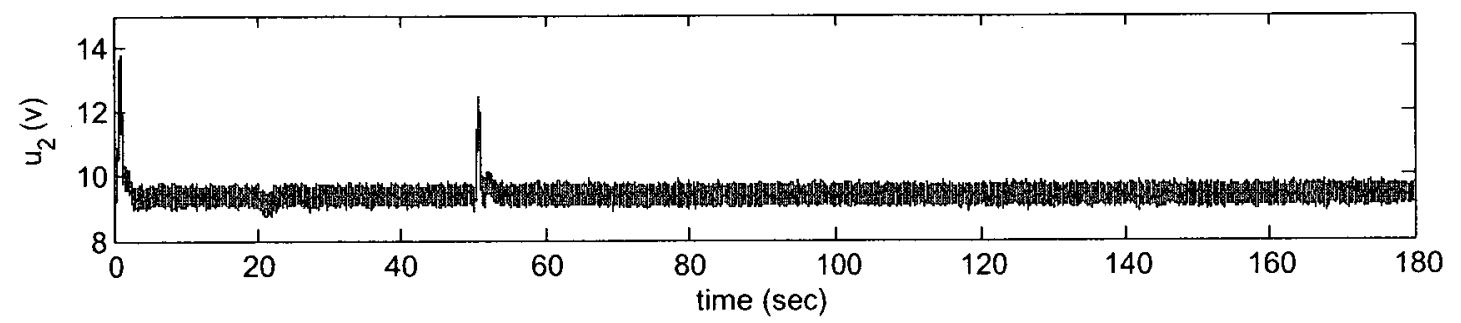

(c)

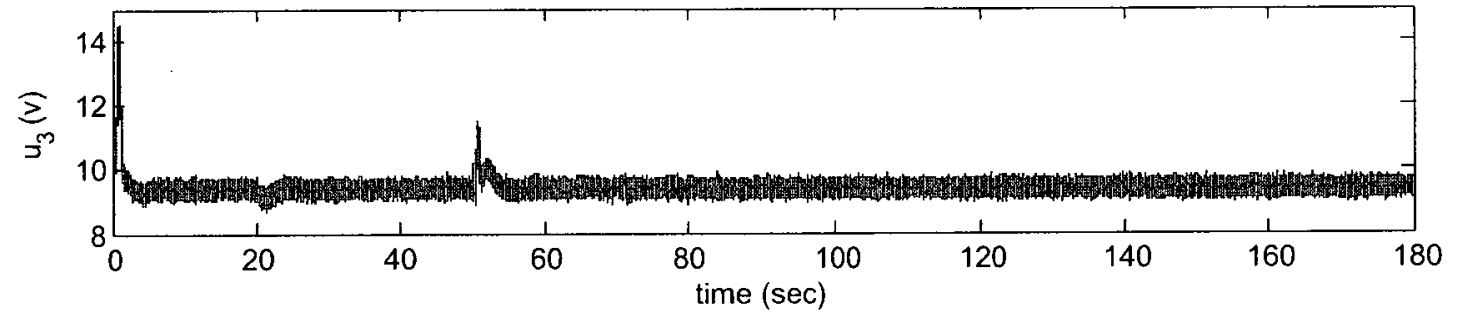

(d)

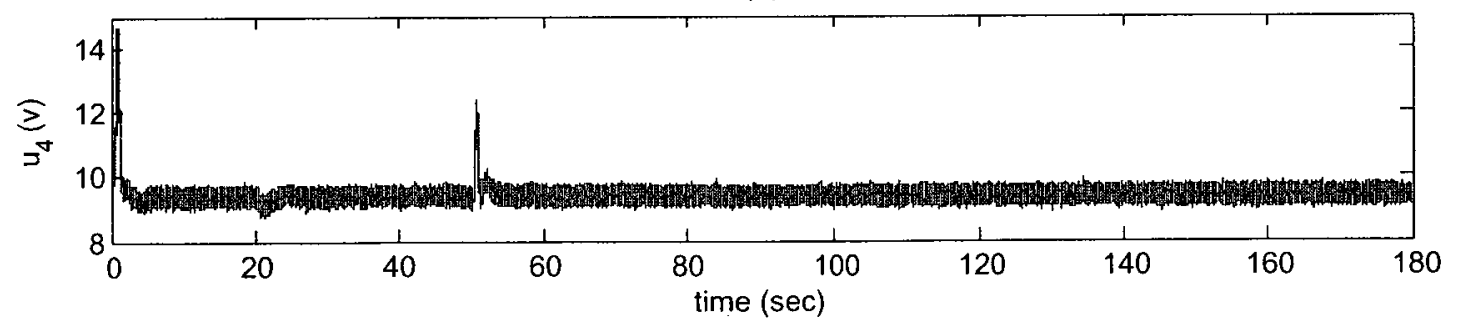

Figure 4.4: Input signals in response to the commanded trajectory corresponding to a $10 \%$ LOE fault in the first actuator and without fault recovery mechanism. 
(a)

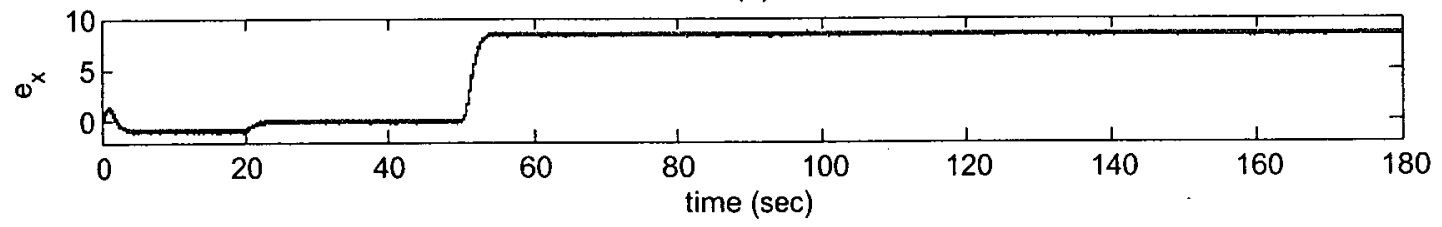

(b)

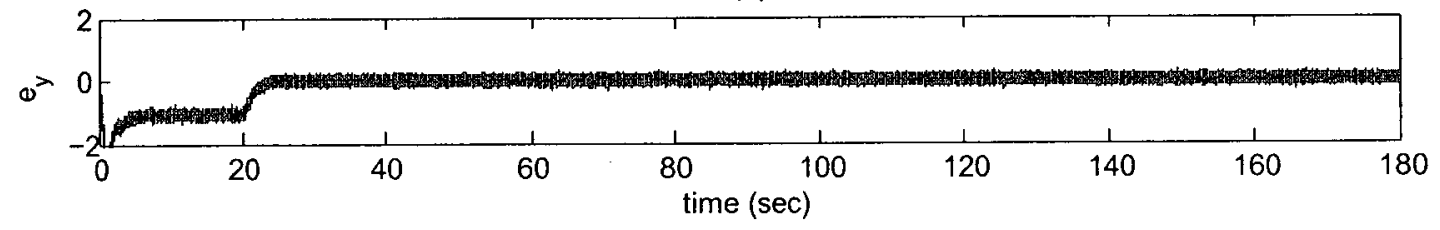

(c)

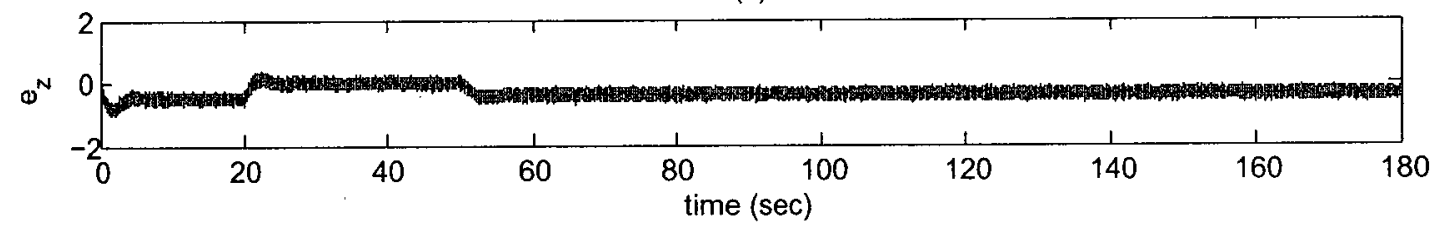

(d)

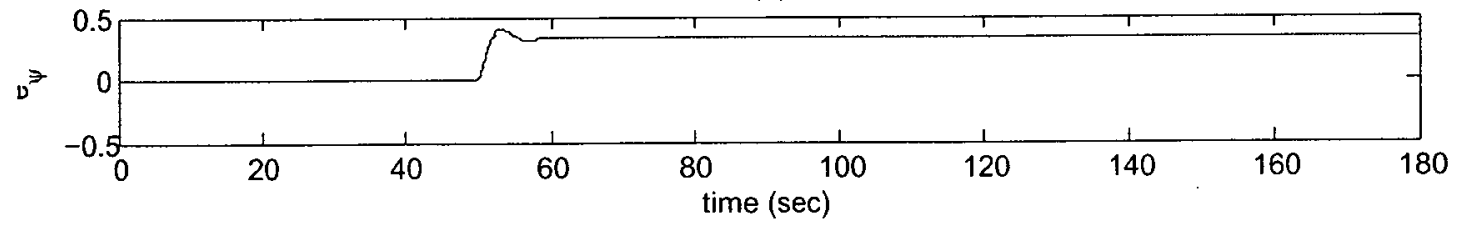

Figure 4.5: Error signals $e_{x}, e_{y}, e_{z}$ measured in meters and $e_{\psi}$ in radians in response to the commanded trajectory corresponding to a $10 \%$ LOE fault in the first actuator and without fault recovery mechanism. 
(a)

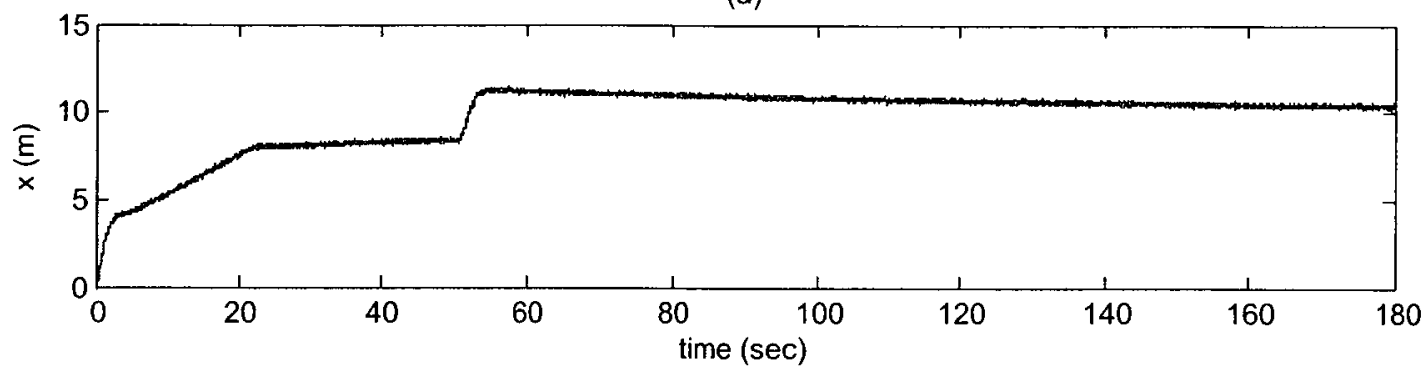

(b)

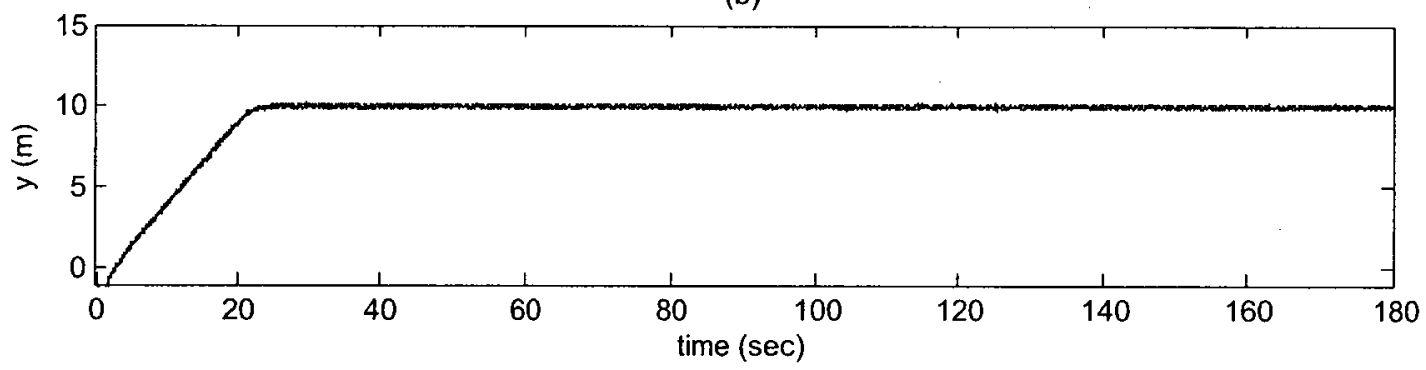

(c)

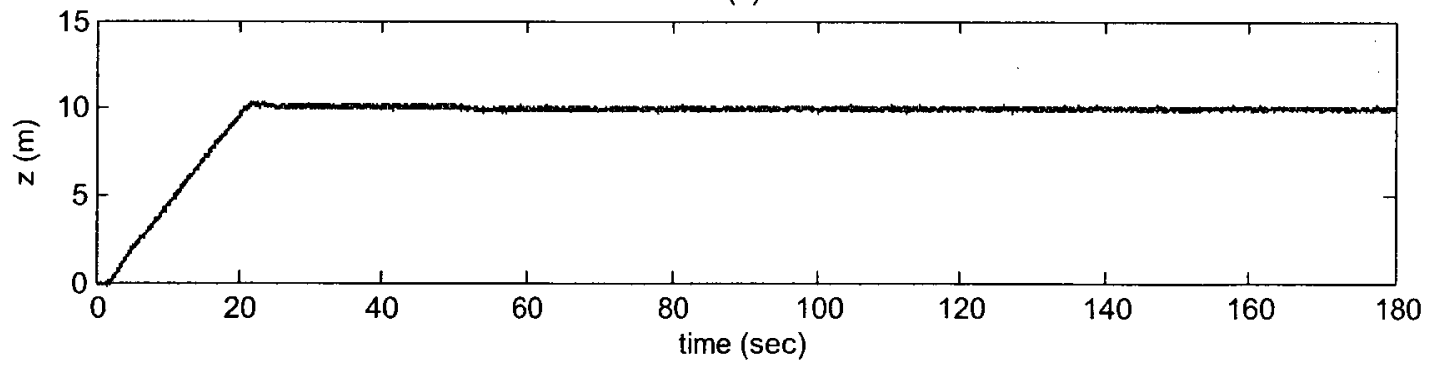

Figure 4.6: Linear position in response to the commanded trajectory corresponding to a $10 \% \mathrm{LOE}$ fault in the first actuator and with the fault recovery mechanism: (a) $\mathrm{x},(\mathrm{b}) \mathrm{y}$ and (c) $\mathrm{z}$, measured in meters. 
(a)

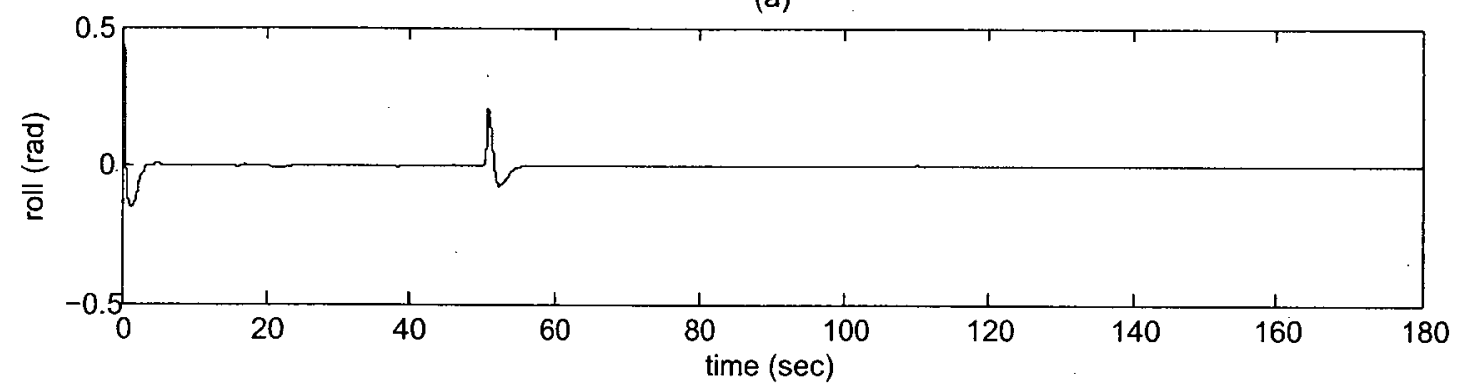

(b)

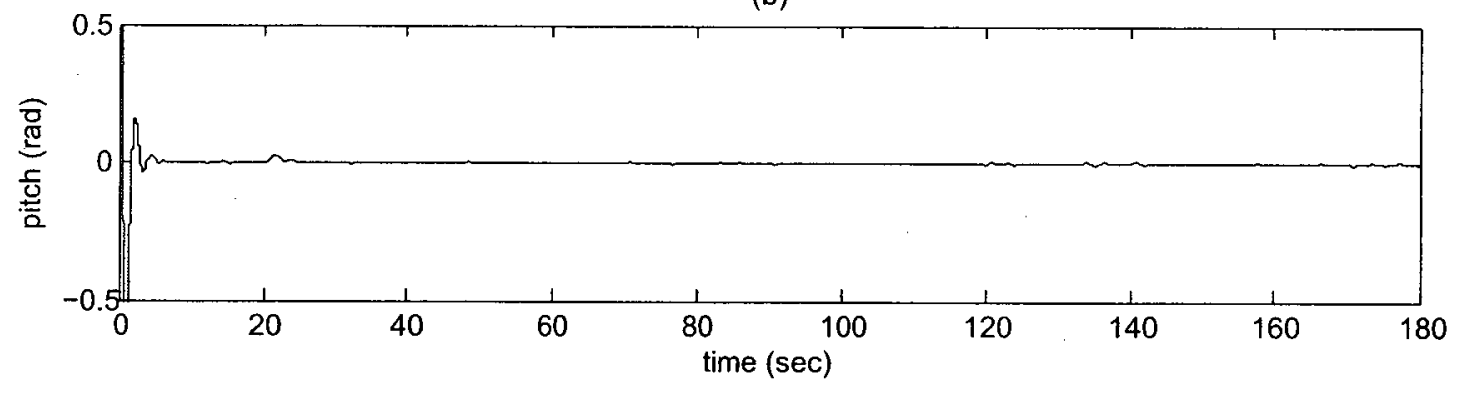

(c)

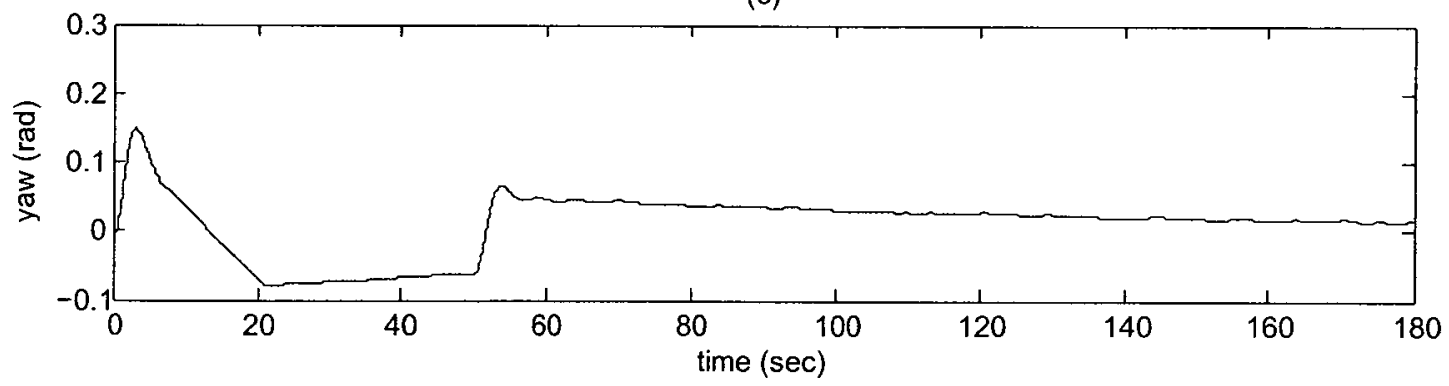

Figure 4.7: Euler angles in response to the commanded trajectory corresponding to a $10 \%$ LOE fault in the first actuator and with the fault recovery mechanism: (a) Roll (rad), (b) Pitch (rad) and (c) Yaw (rad). 
(a)

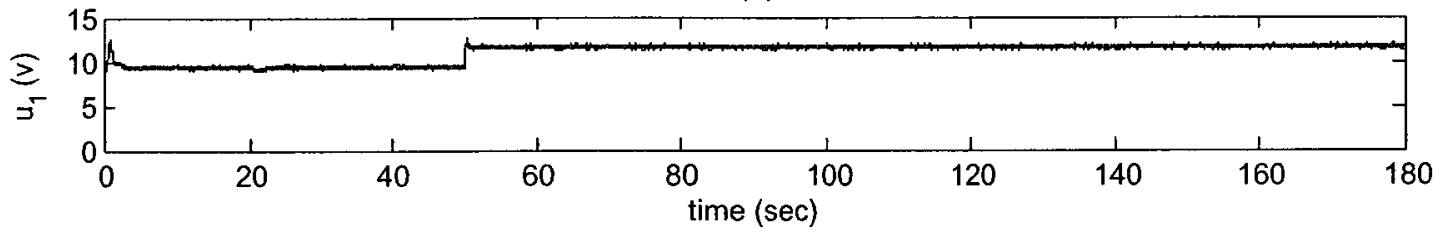

(b)

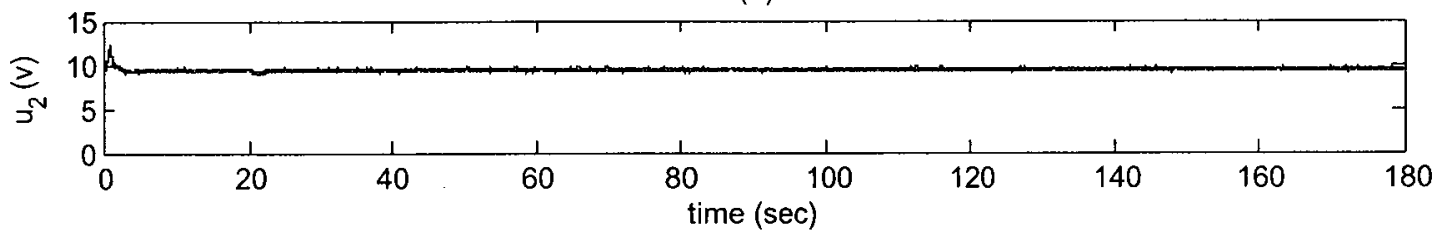

(c)

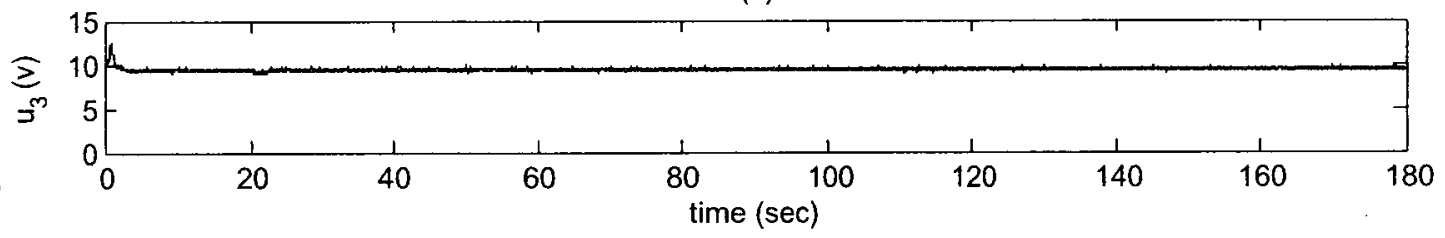

(d)

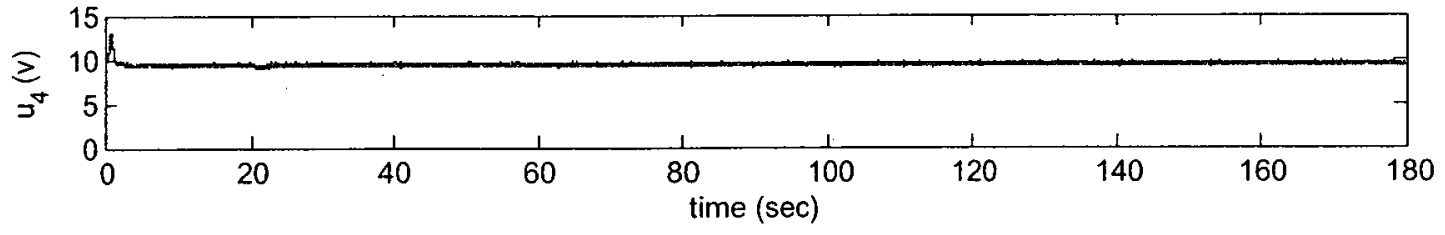

Figure 4.8: Input signals in response to the commanded trajectory corresponding to a $10 \%$ LOE fault in the first actuator with the fault recovery mechanism. 
(a)

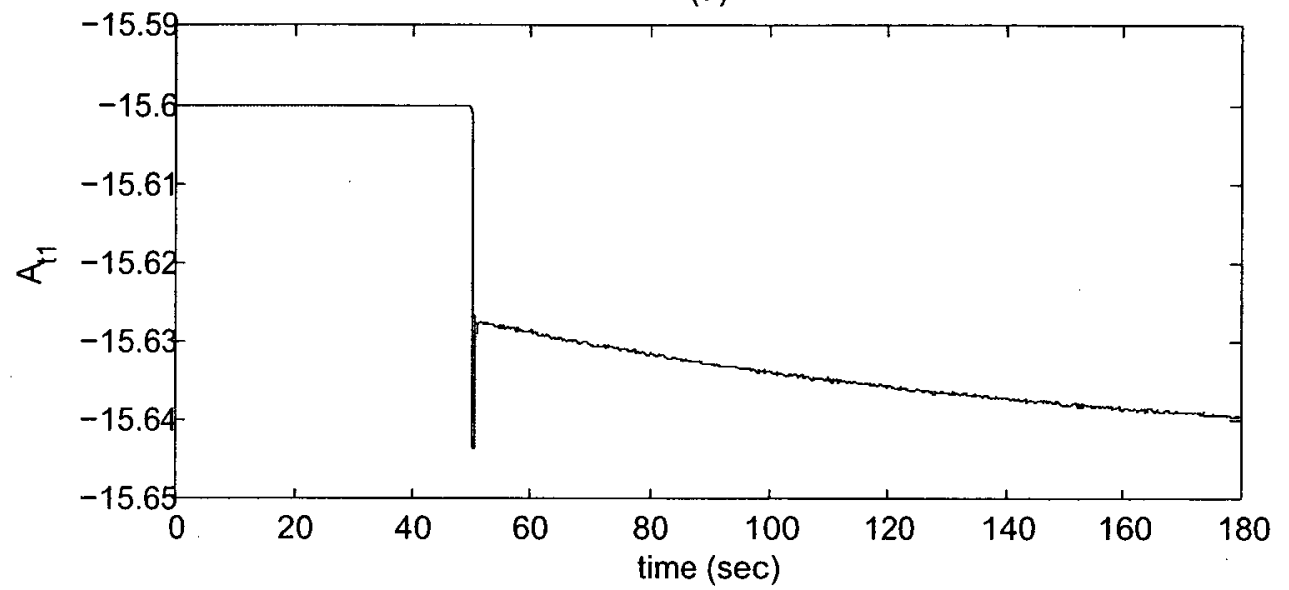

(b)

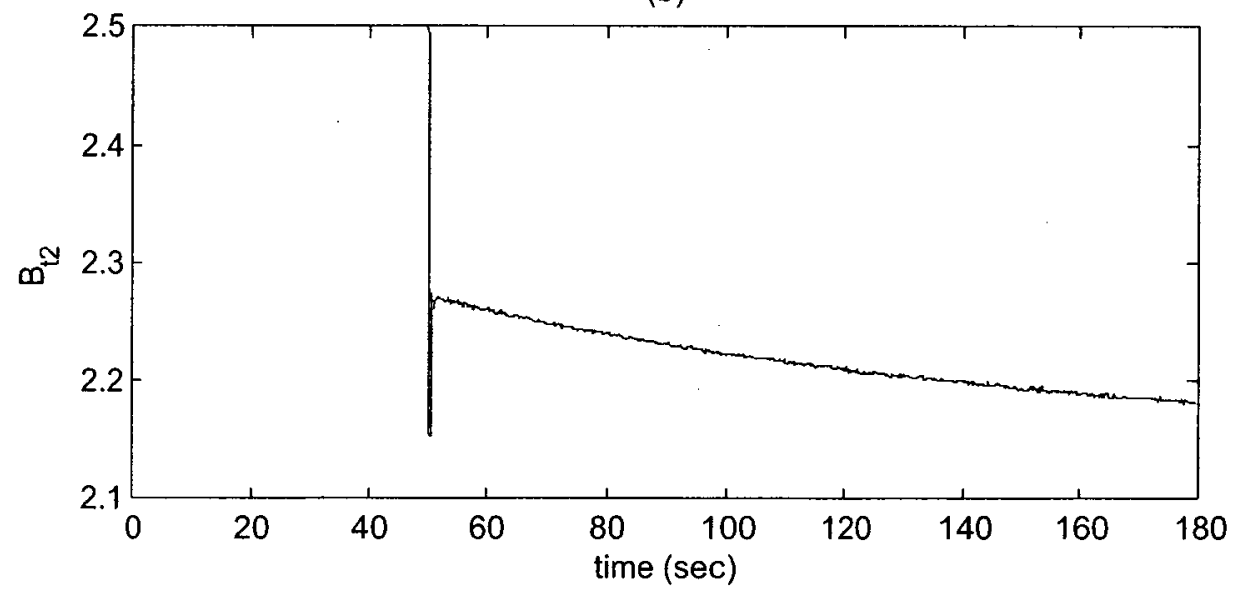

Figure 4.9: Estimated parameters in response to the commanded trajectory corresponding to a $10 \% \mathrm{LOE}$ fault in the first actuator with the fault recovery mechanism: (a) $A_{t 1}$ and (b) $B_{t 1}$ 
(a)

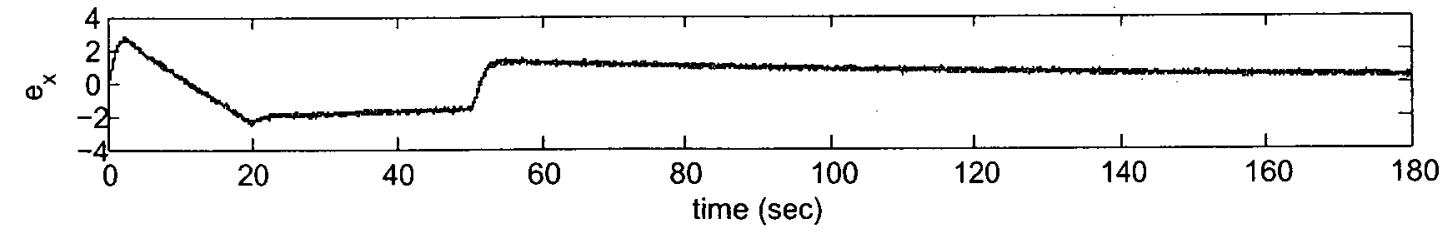

(b)

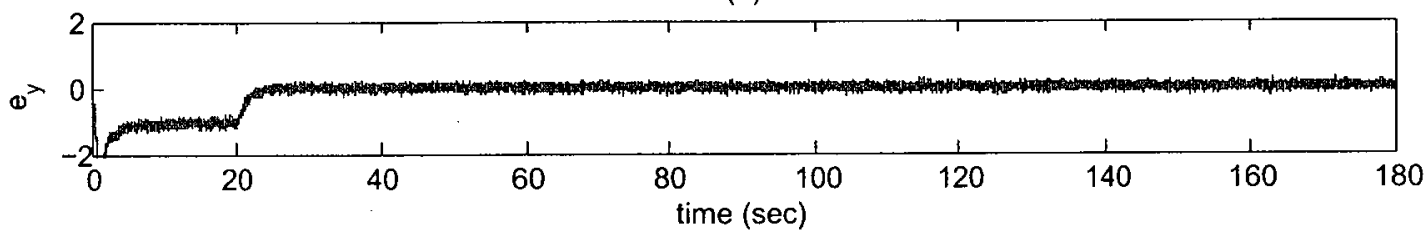

(c)

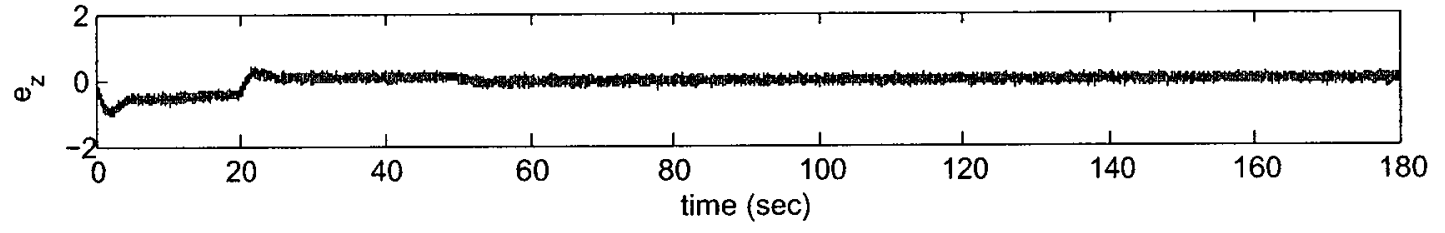

(d)

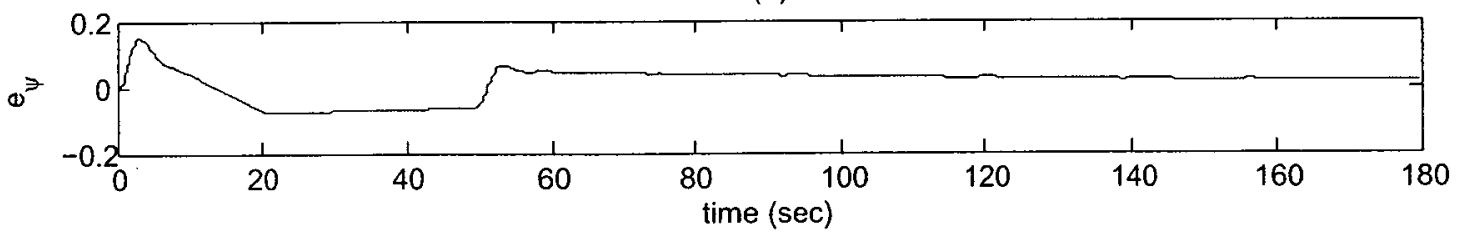

Figure 4.10: Error signals $e_{x}, e_{y}, e_{z}$ measured in meters and $e_{\psi}$ in radians in response to the commanded trajectory corresponding to a $10 \%$ LOE fault in the first actuator with the fault recovery mechanism. 
(a)

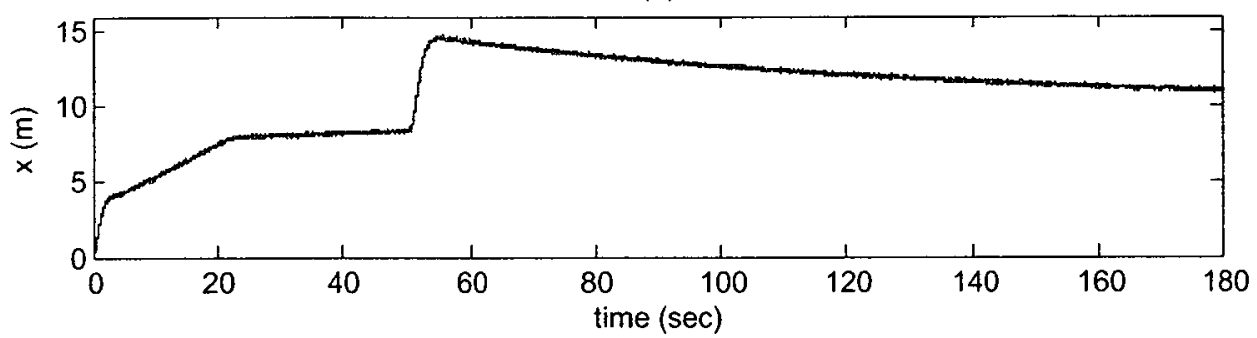

(b)

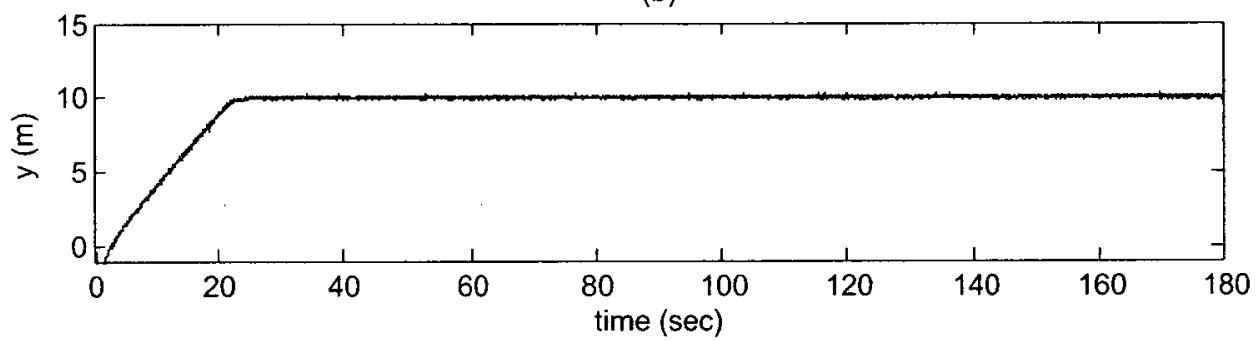

(c)

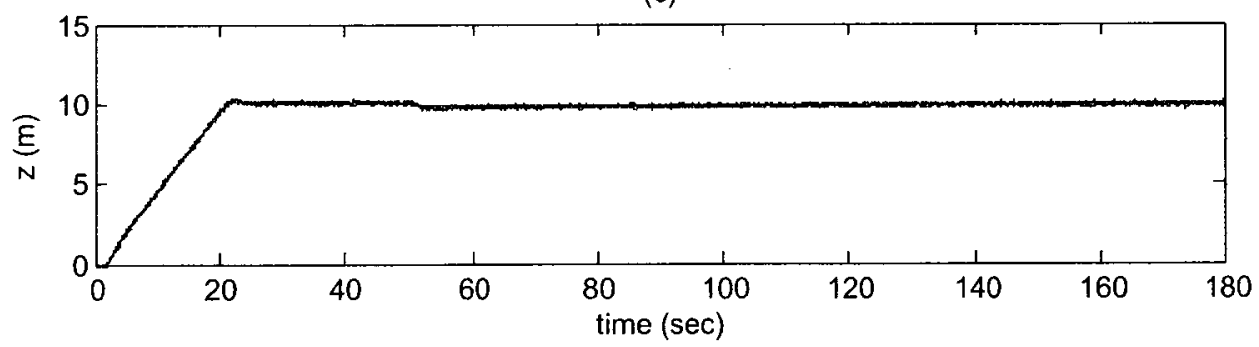

Figure 4.11: Linear position in response to the commanded trajectory corresponding to a $25 \%$ LOE fault in the first actuator with the fault recovery mechanism: (a) $\mathrm{x}$, (b) y and (c) $\mathrm{z}$, measured in meters. 
(a)

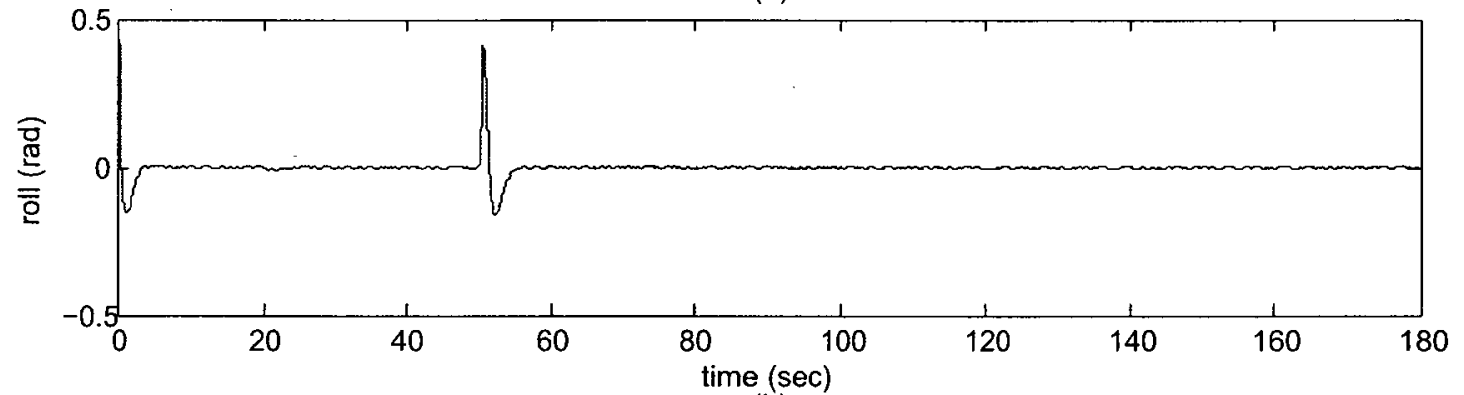

(b)

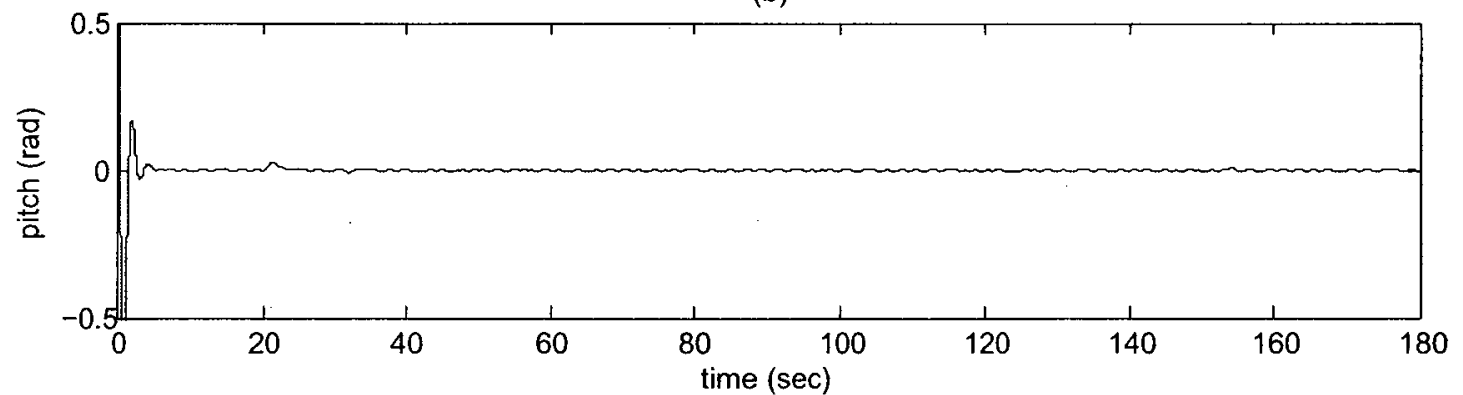

(c)

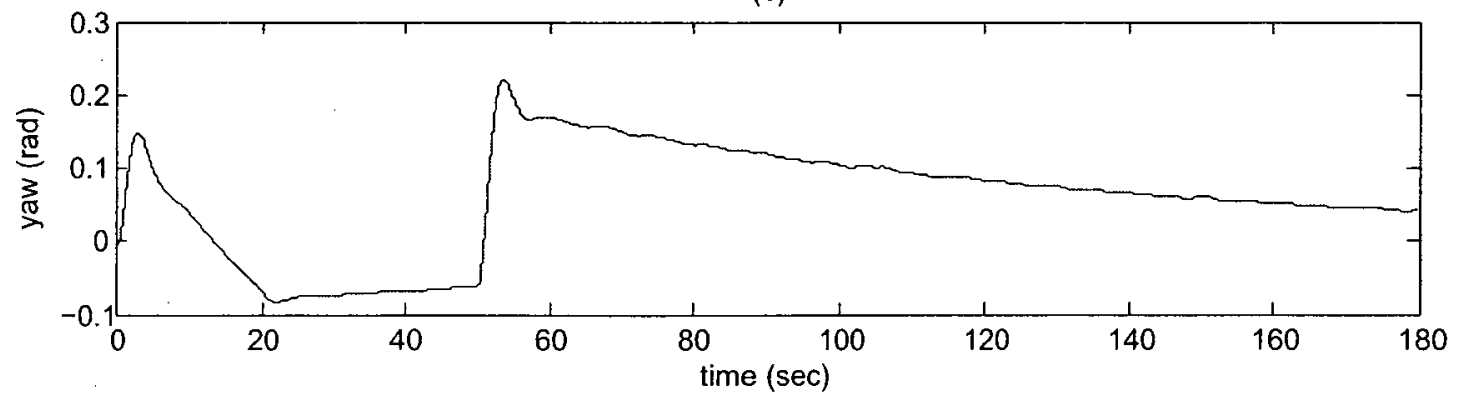

Figure 4.12: Euler angles in response to the commanded trajectory corresponding to a $25 \%$ LOE fault in the first actuator with the fault recovery mechanism: (a) Roll (rad), (b) Pitch (rad) and (c) Yaw (rad). 
(a)

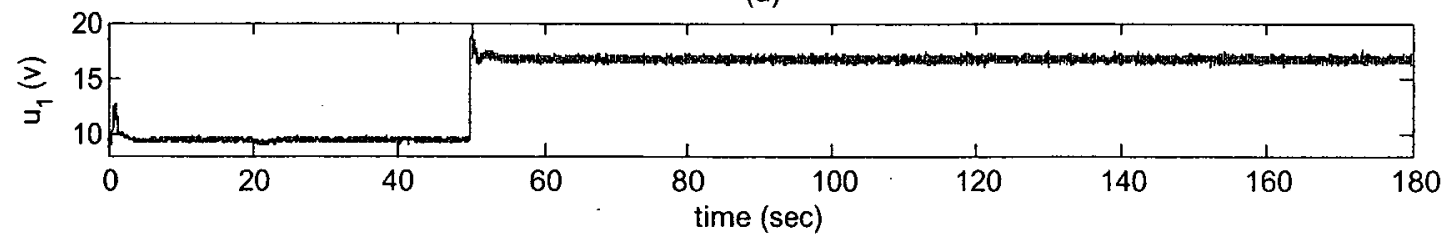

(b)

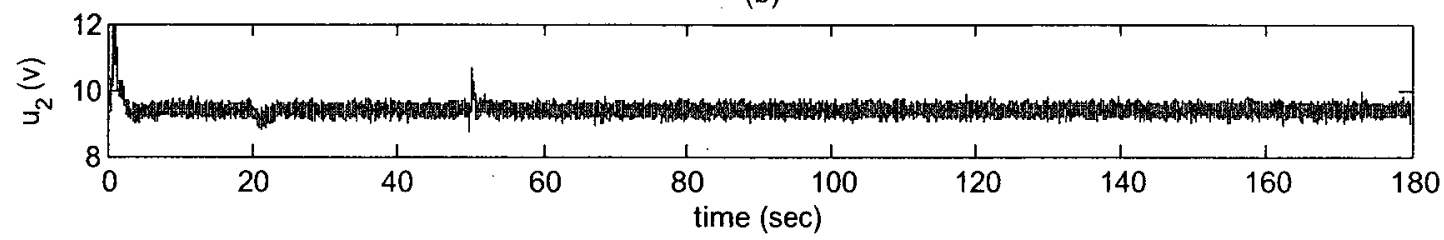

(c)

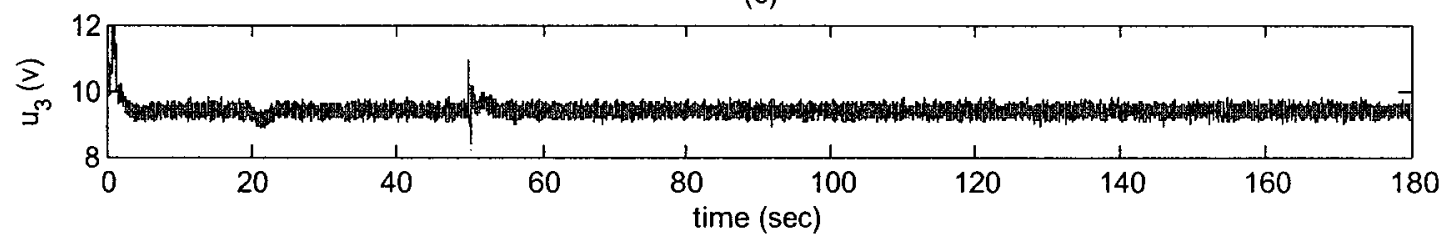

(d)

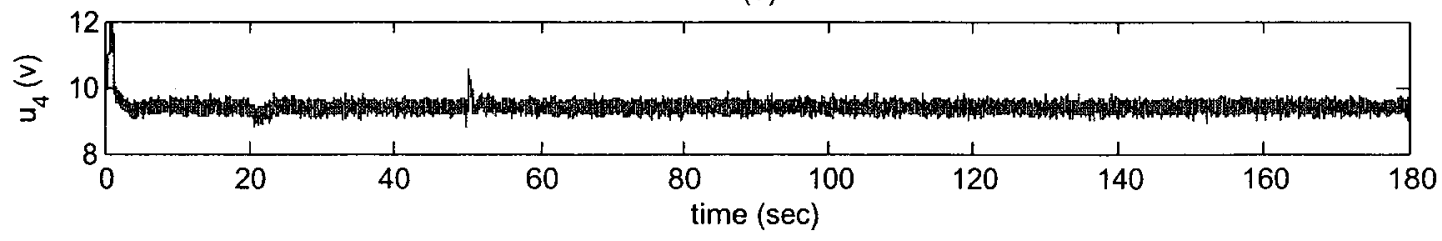

Figure 4.13: Input signals in response to the commanded trajectory corresponding to a $25 \% \mathrm{LOE}$ fault in the first actuator with the fault recovery mechanism. 
(a)

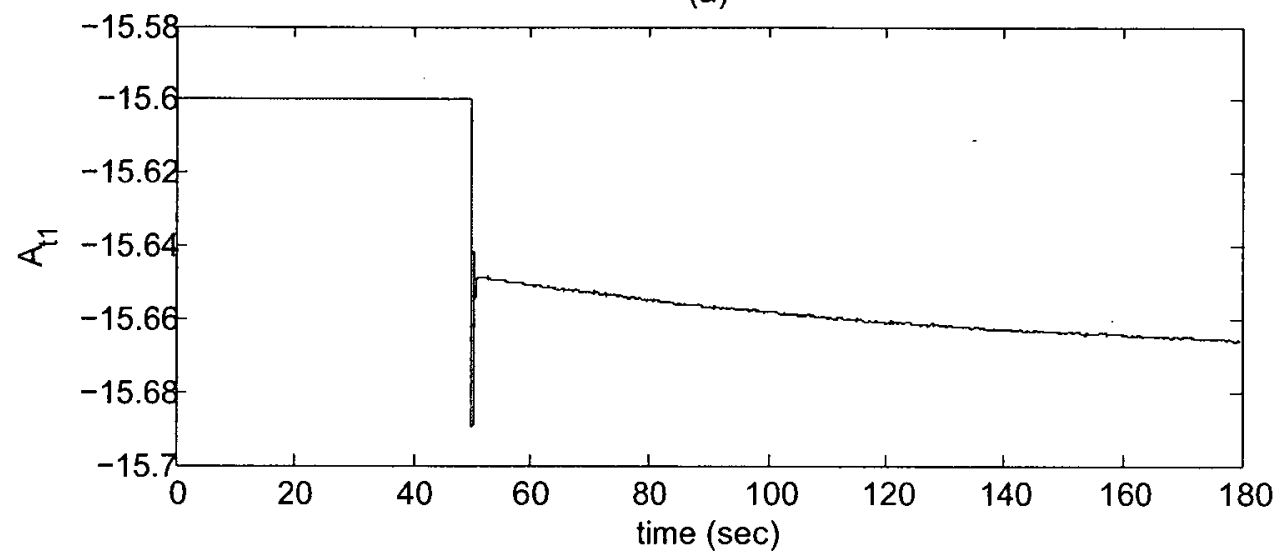

(b)

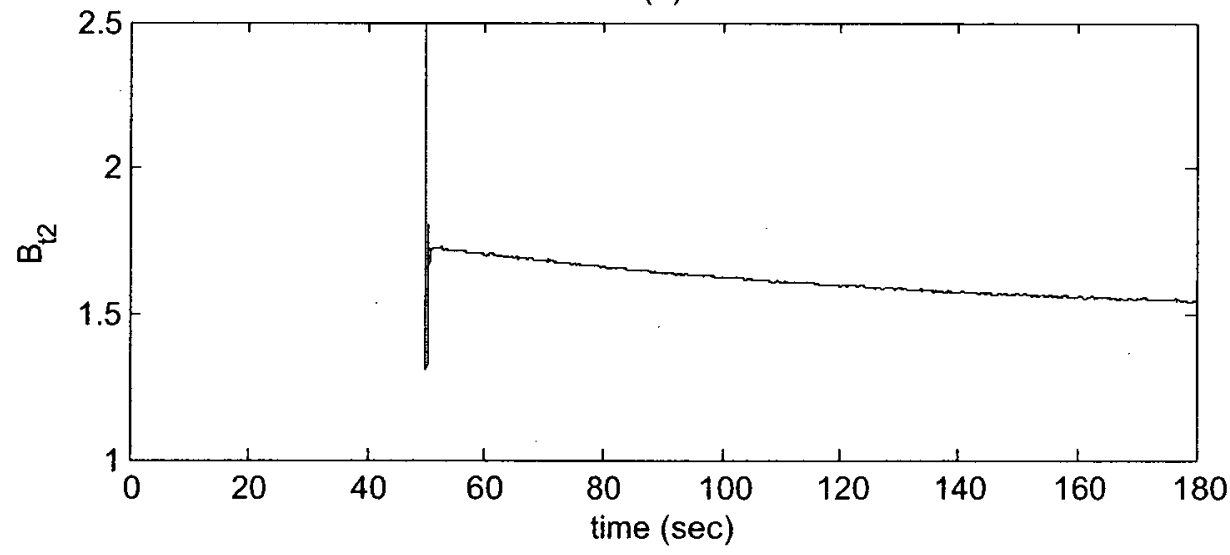

Figure 4.14: Estimated parameters in response to the commanded trajectory corresponding to a $\% 25 \mathrm{LOE}$ fault in the first actuator with the fault recovery mechanism: (a) $A_{t 1}$ and (b) $B_{t 1}$ 
(a)

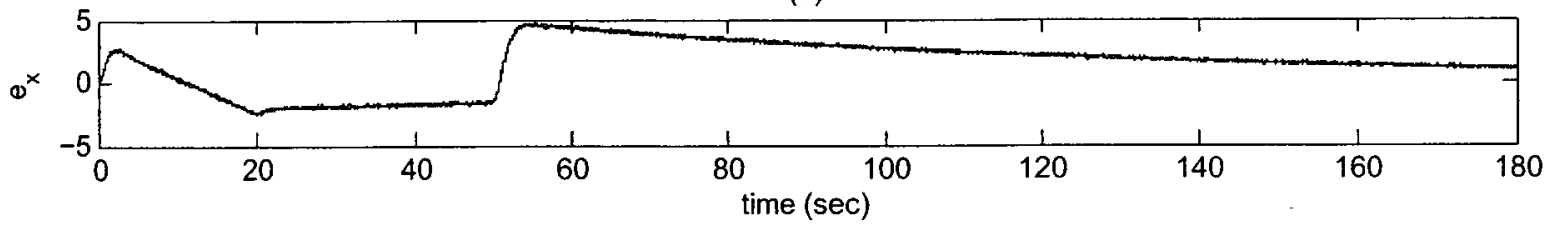

(b)

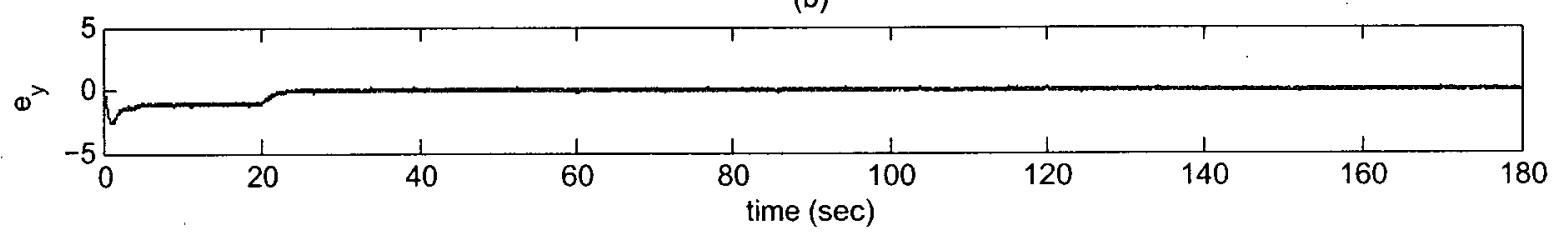

(c)

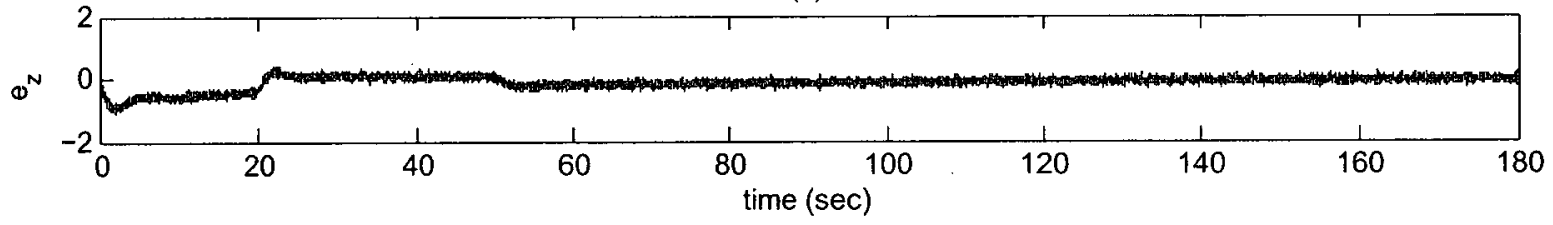

(d)

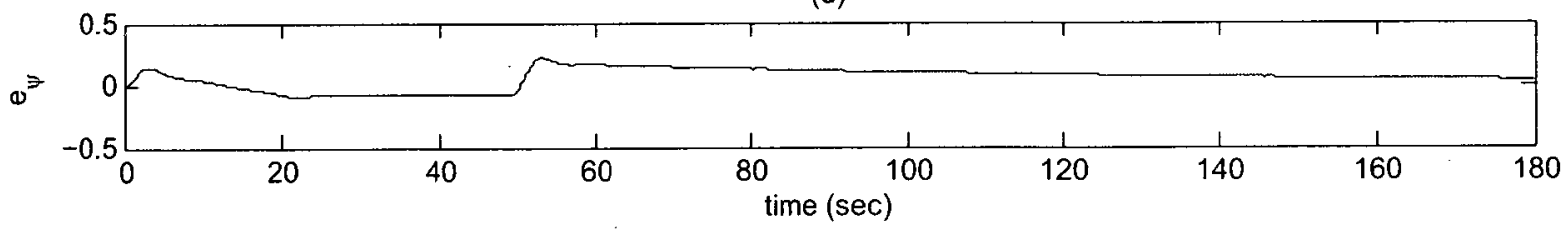

Figure 4.15: Error signals $e_{x}, e_{y}, e_{z}$ measured in meters and $e_{\psi}$ in radians in response to the commanded trajectory corresponding to a $25 \% \mathrm{LOE}$ fault in the first actuator with the fault recovery mechanism. 
(a)

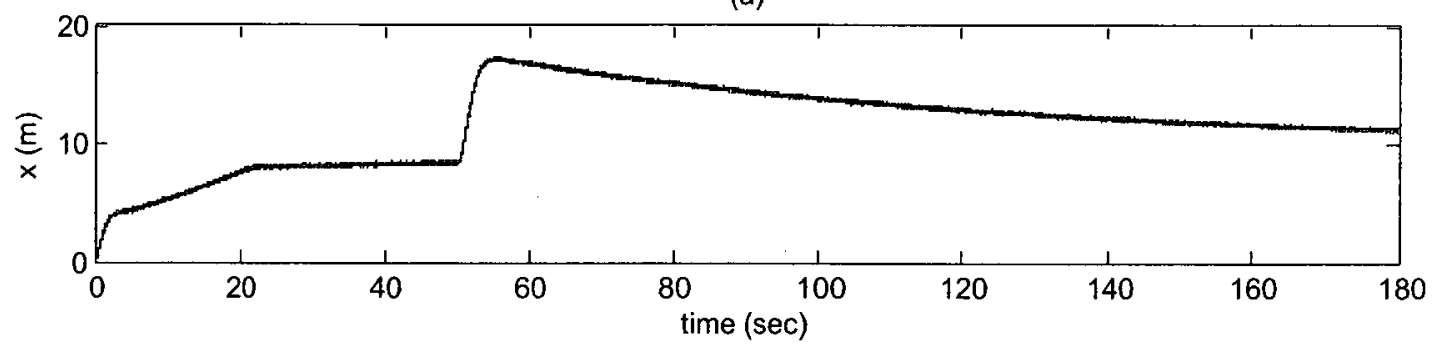

(b)

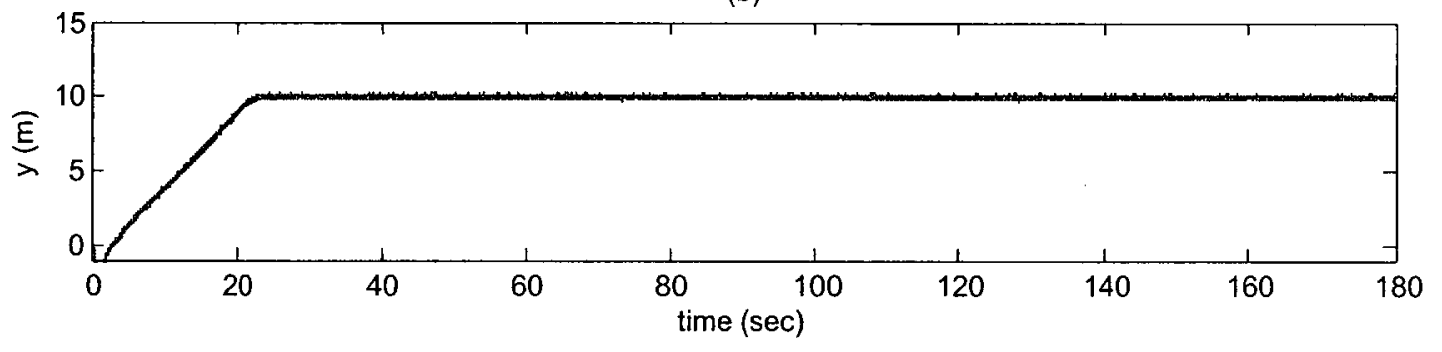

(c)

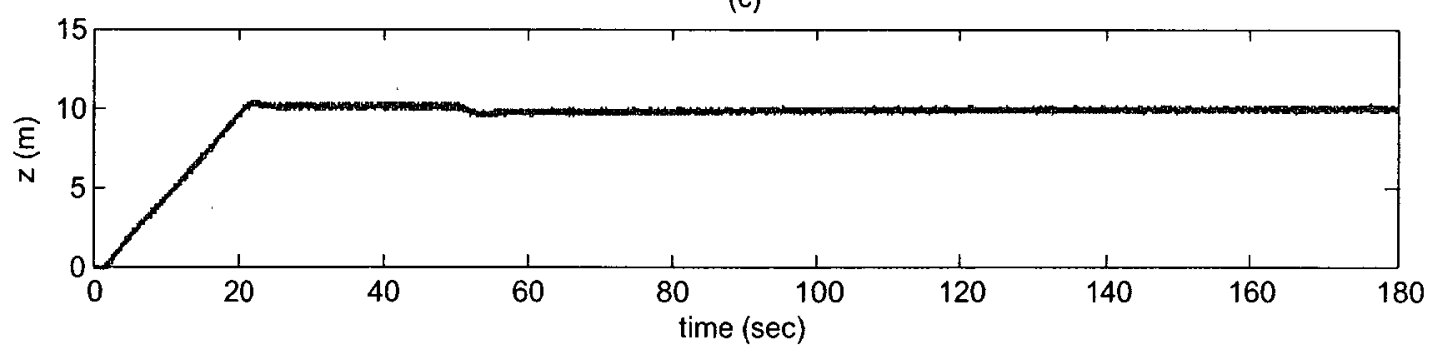

Figure 4.16: Linear position in response to the commanded trajectory corresponding to a $50 \%$ LOE fault in the first actuator with the fault recovery mechanism: (a) $\mathrm{x}$, (b) y and (c) $\mathrm{z}$, measured in meters. 
(a)

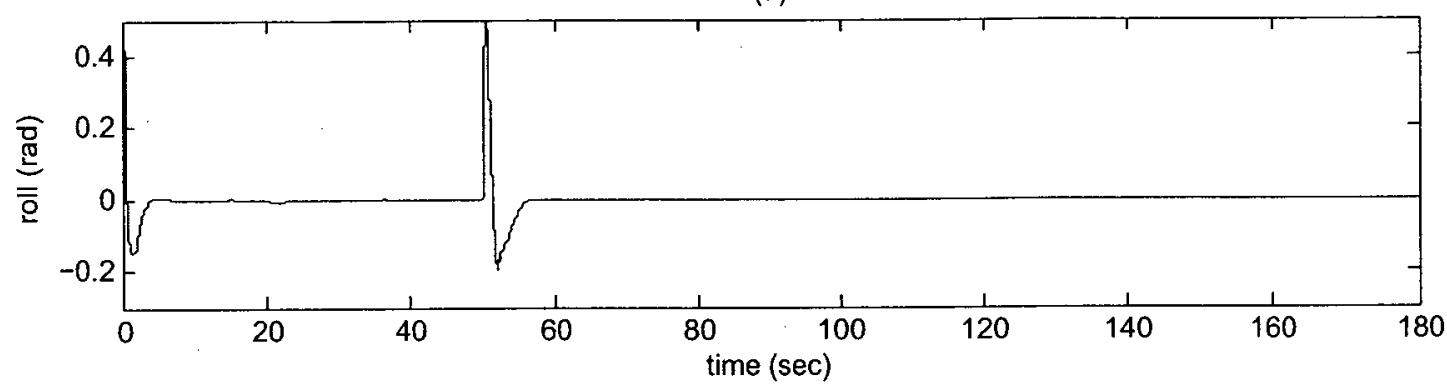

(b)

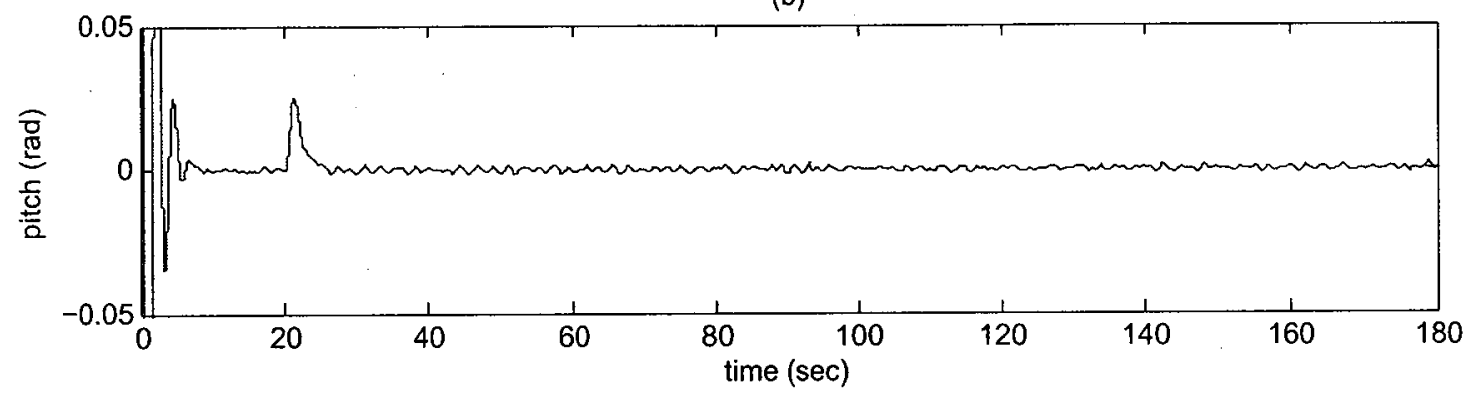

(c)

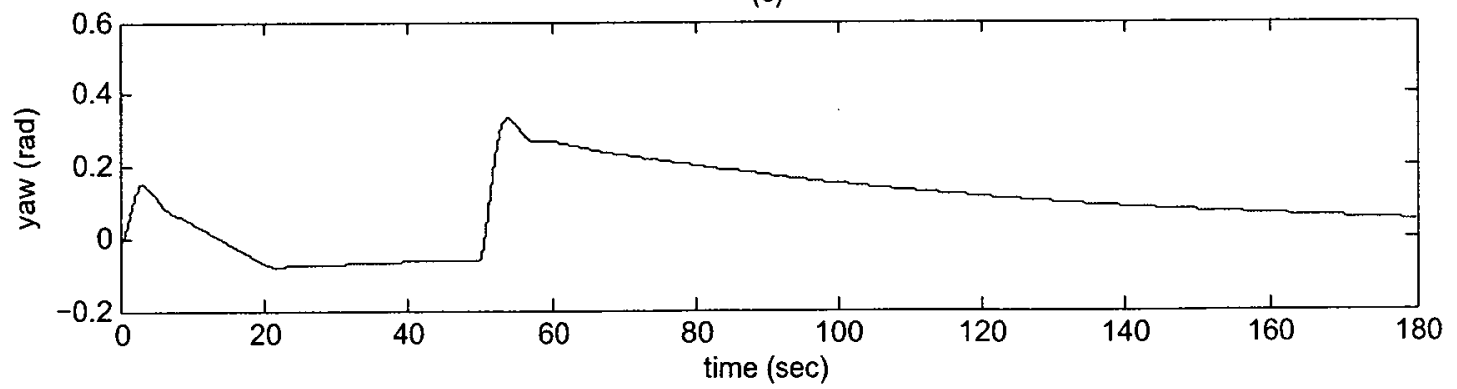

Figure 4.17: Euler angles in response to the commanded trajectory corresponding to a $50 \%$ LOE fault in the first actuator with the fault recovery mechanism: (a) Roll (rad), (b) Pitch (rad) and (c) Yaw (rad). 
(a)

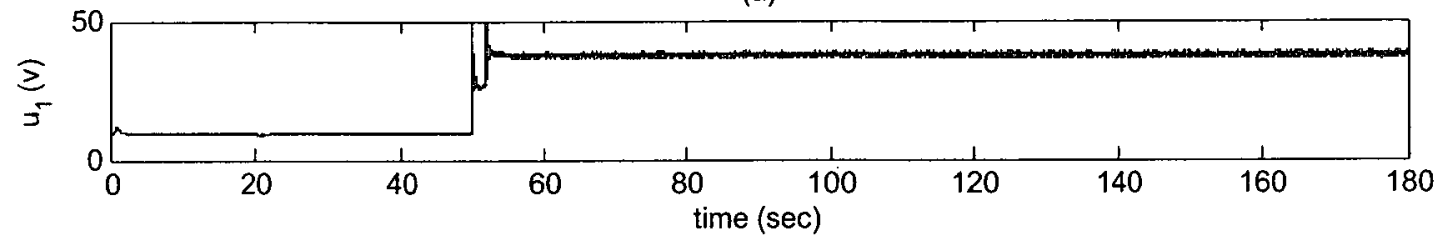

(b)

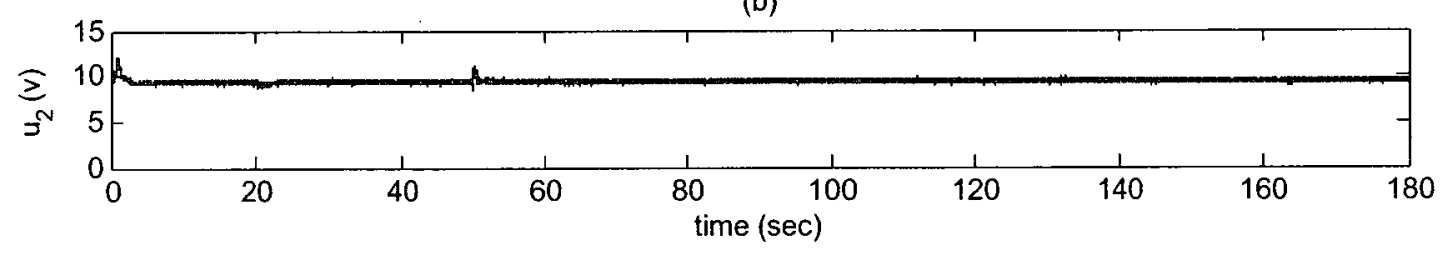

(c)

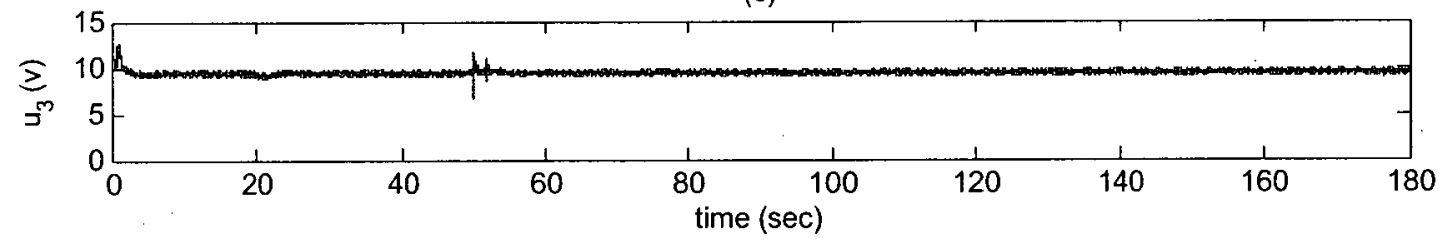

(d)

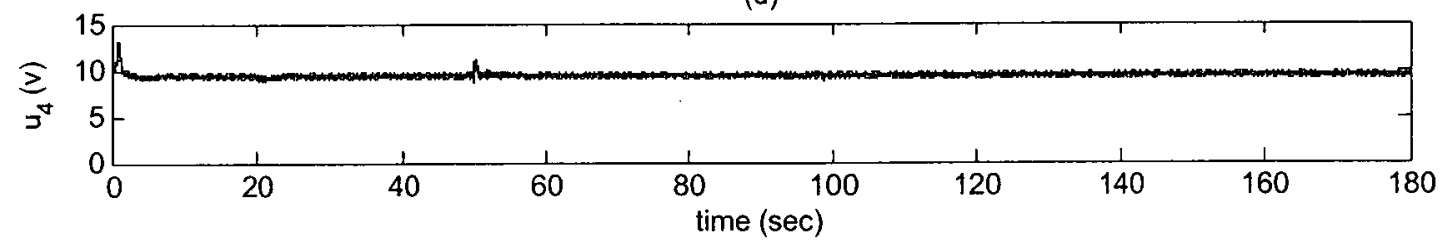

Figure 4.18: Input signals in response to the commanded trajectory corresponding to a $50 \%$ LOE fault in the first actuator with the fault recovery mechanism. 
(a)

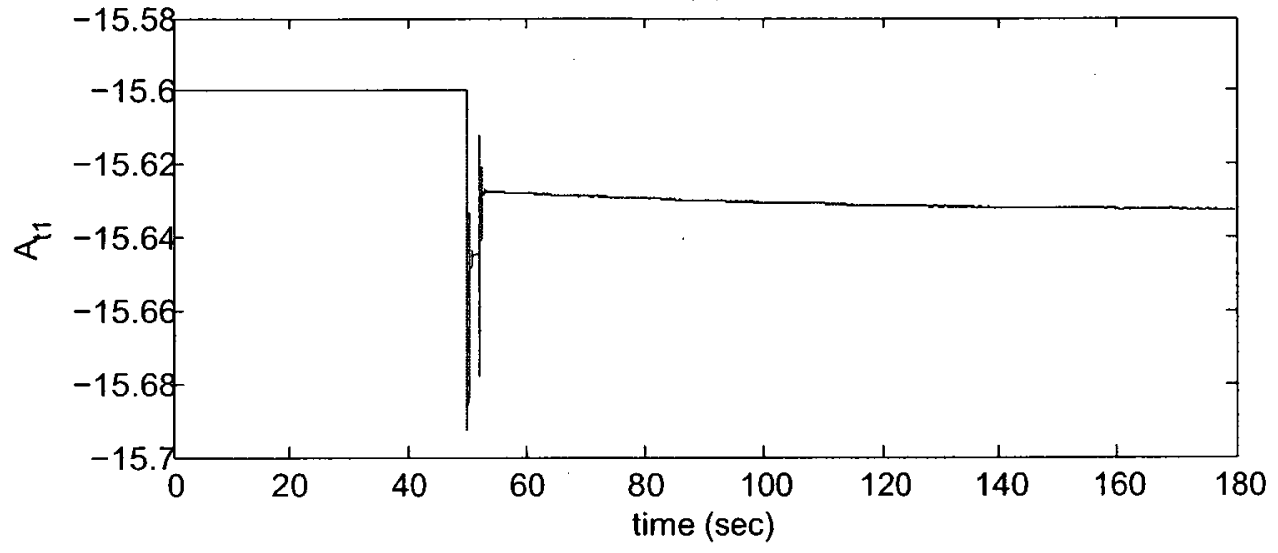

(b)

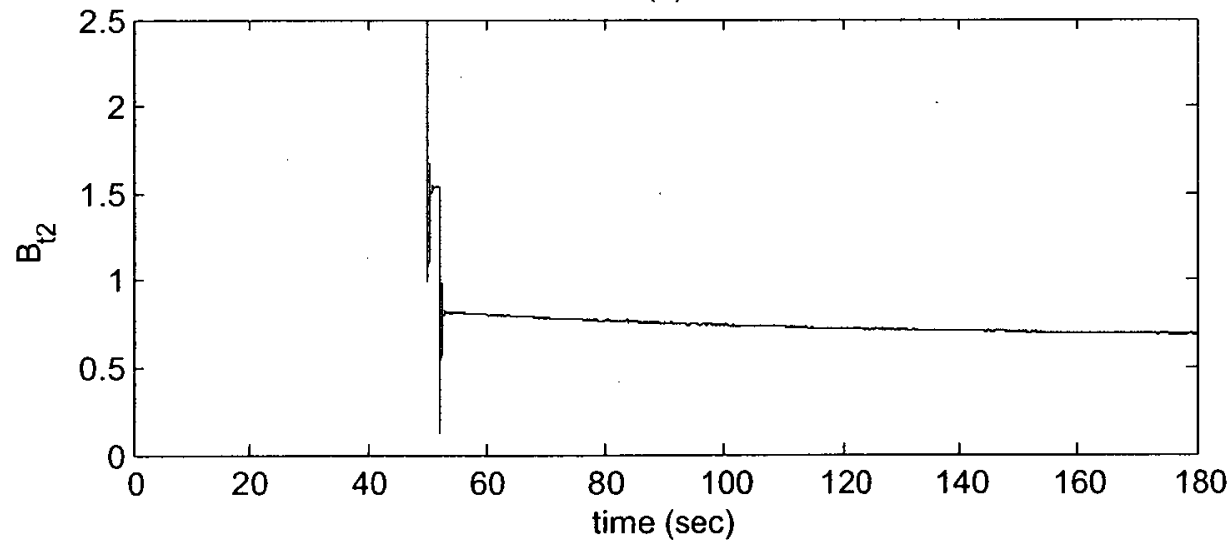

Figure 4.19: Estimated parameters in response to the commanded trajectory corresponding to a $50 \% \mathrm{LOE}$ fault in the first actuator with the fault recovery mechanism: (a) $A_{t 1}$ and (b) $B_{t 1}$ 
(a)

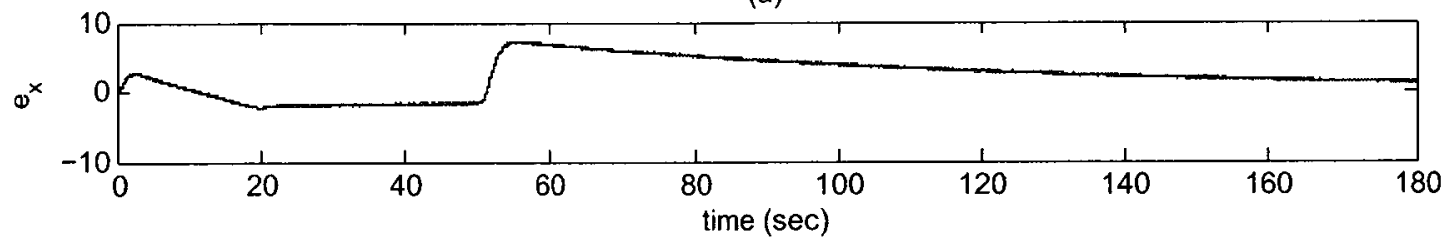

(b)

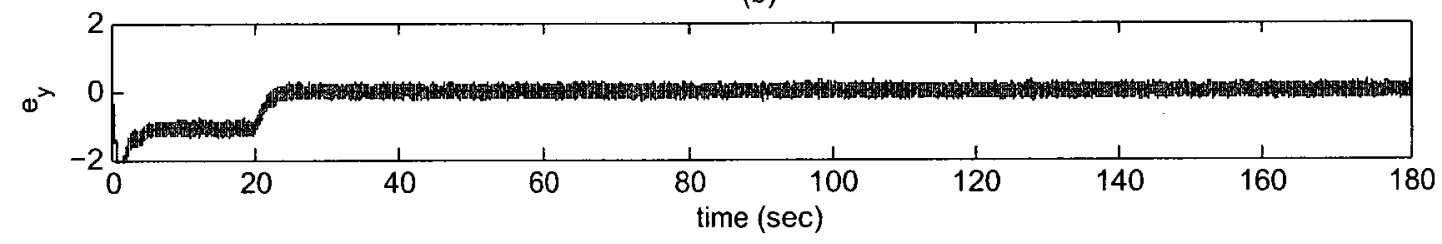

(c)

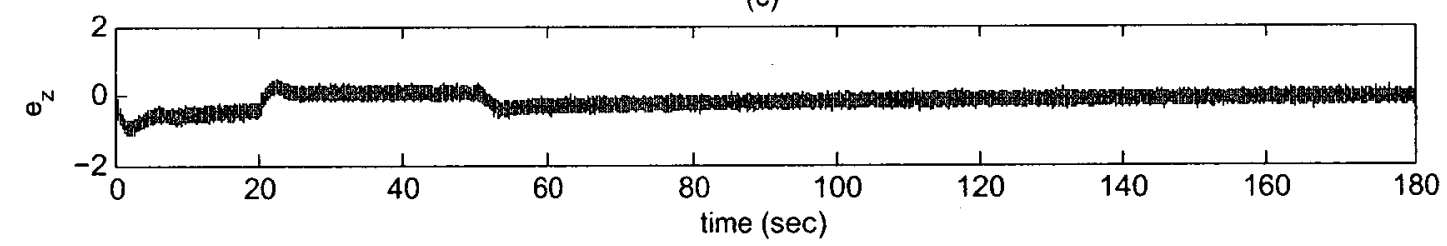

(d)

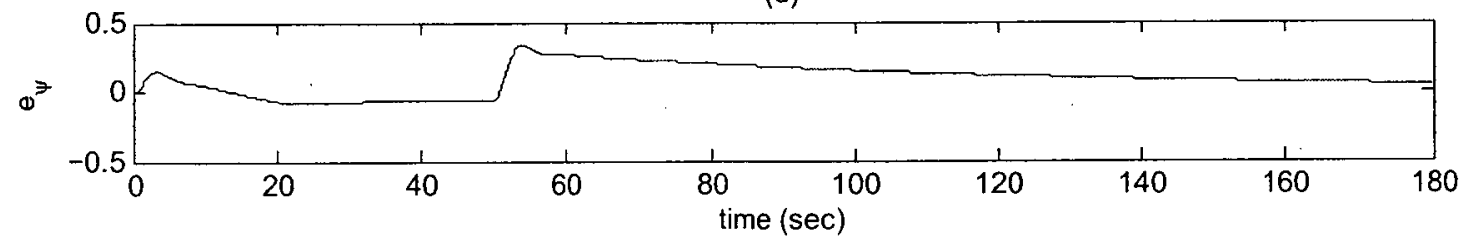

Figure 4.20: Error signals $e_{x}, e_{y}, e_{z}$ measured in meters and $e_{\psi}$ in radians in response to the commanded trajectory corresponding to a $50 \%$ LOE fault in the first actuator with the fault recovery mechanism. 
(a)

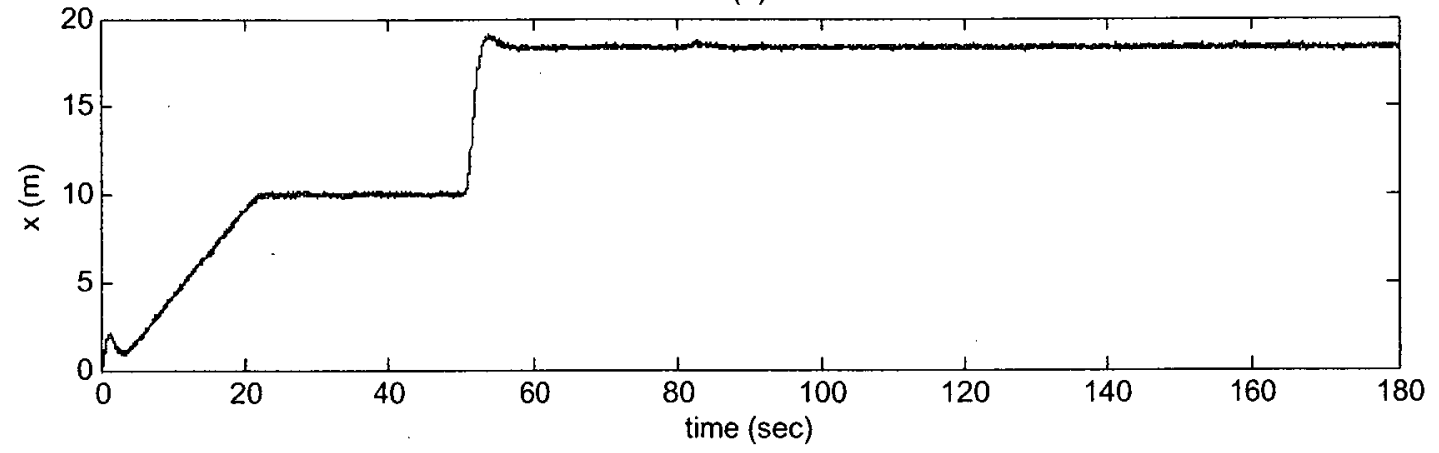

(b)

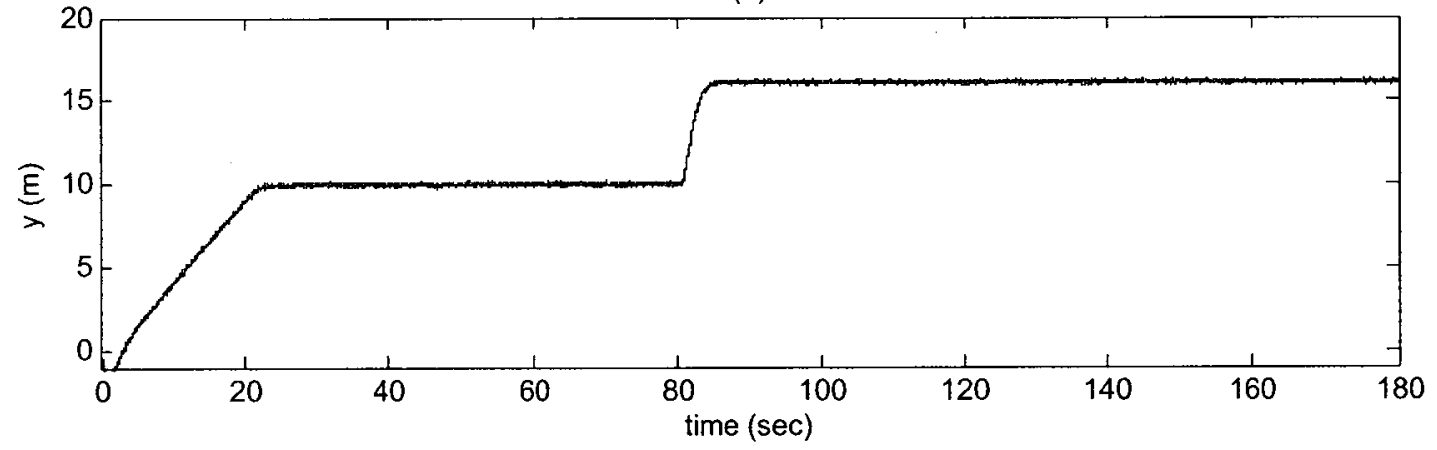

(c)

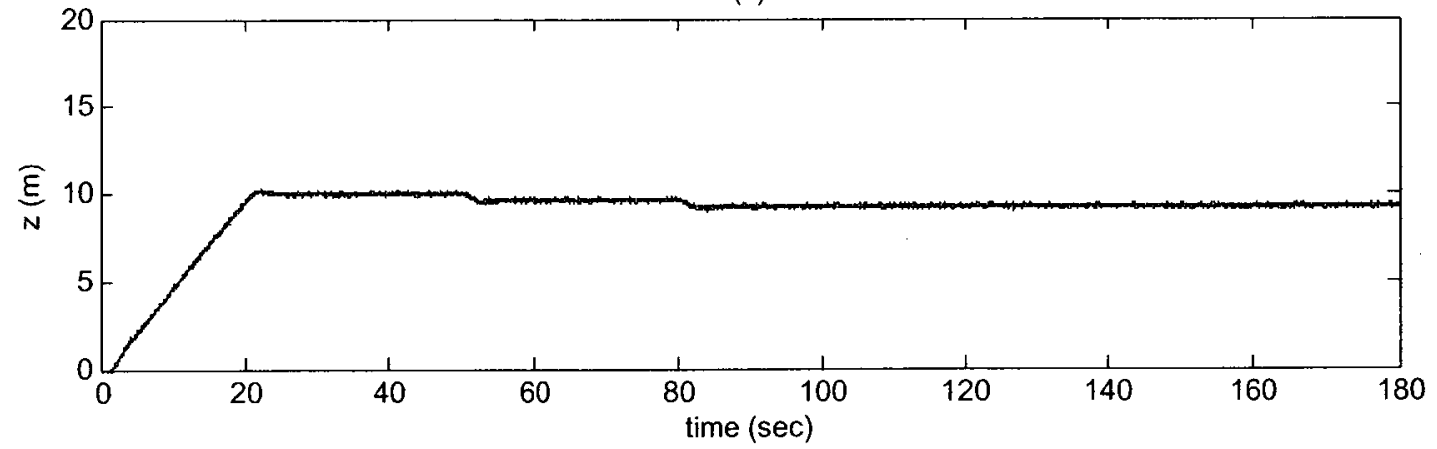

Figure 4.21: Linear position in response to the commanded trajectory corresponding to a $10 \%$ LOE fault in the first and 4 th actuator and without the fault recovery mechanism: (a) $\mathrm{x}$, (b) y and (c) z, measured in meters. 
(a)

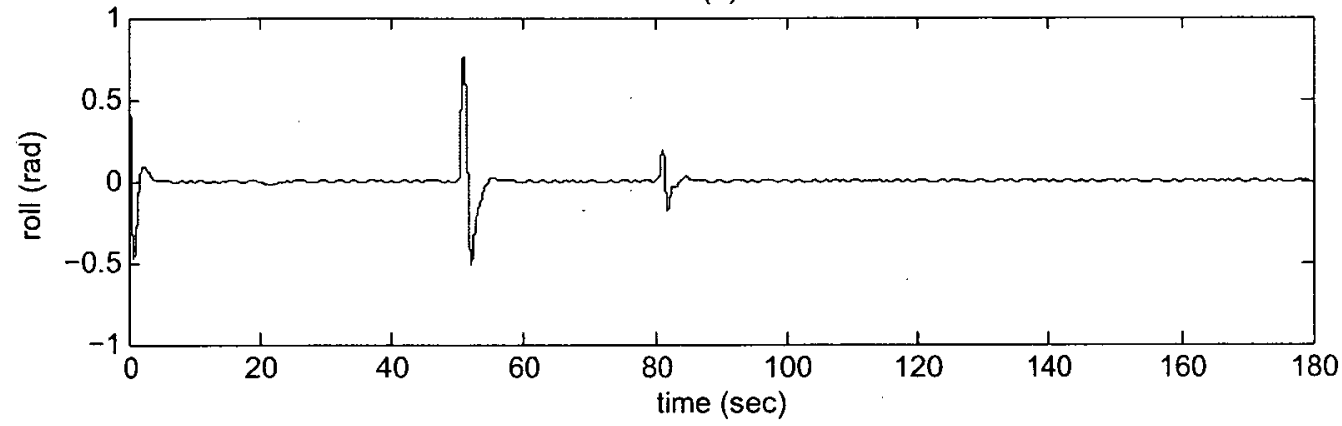

(b)

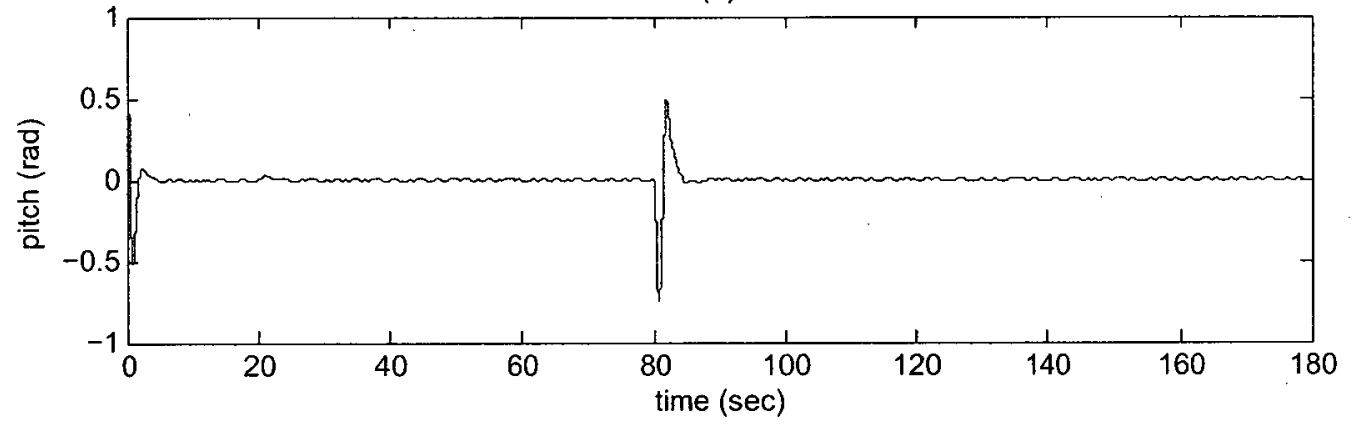

(c)

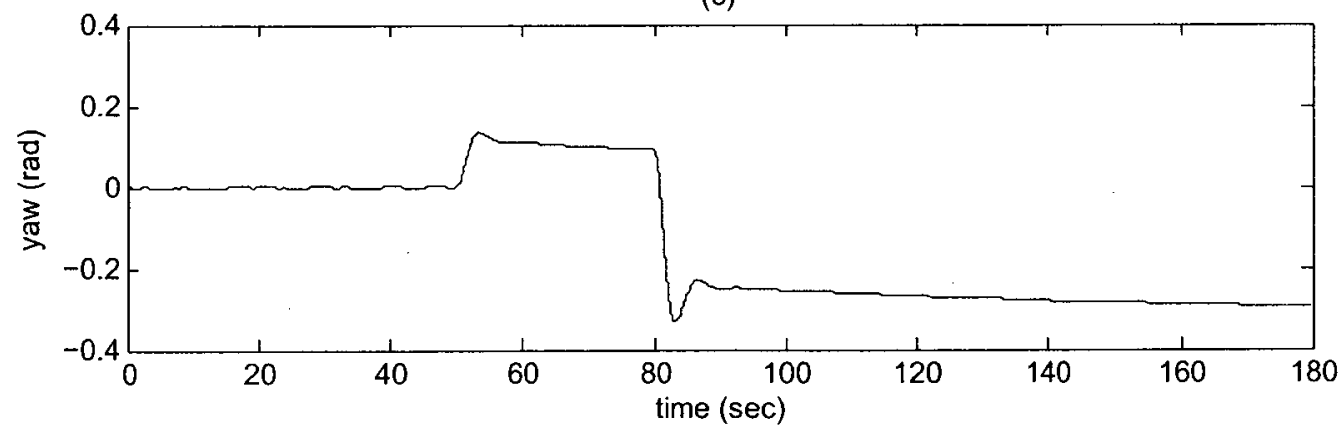

Figure 4.22: Euler angles in response to the commanded trajectory corresponding to a $10 \%$ LOE fault in the first and 4 th actuators and without the fault recovery mechanism: (a) Roll (rad), (b) Pitch (rad) and (c) Yaw (rad). 
(a)

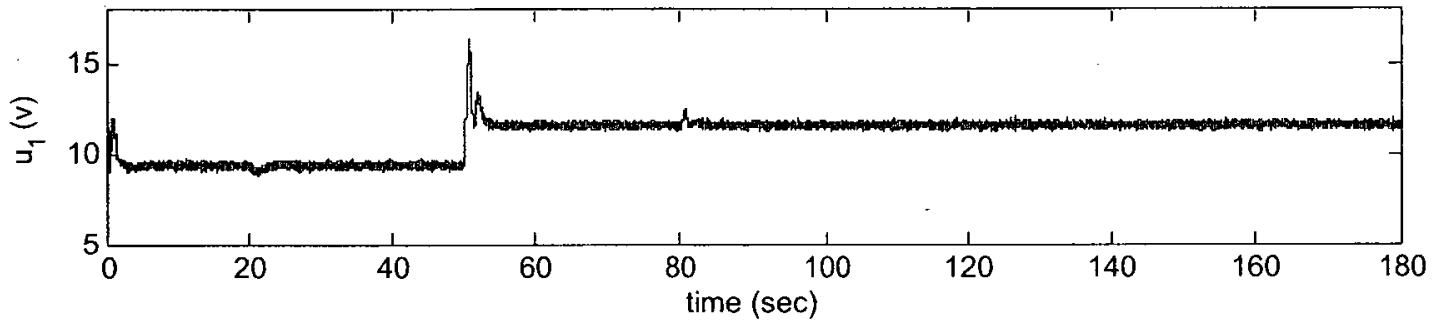

(b)

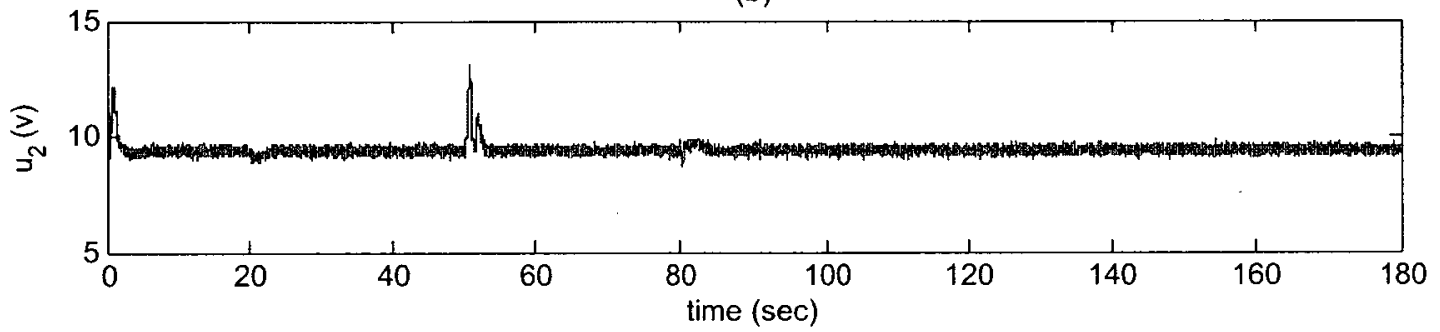

(c)

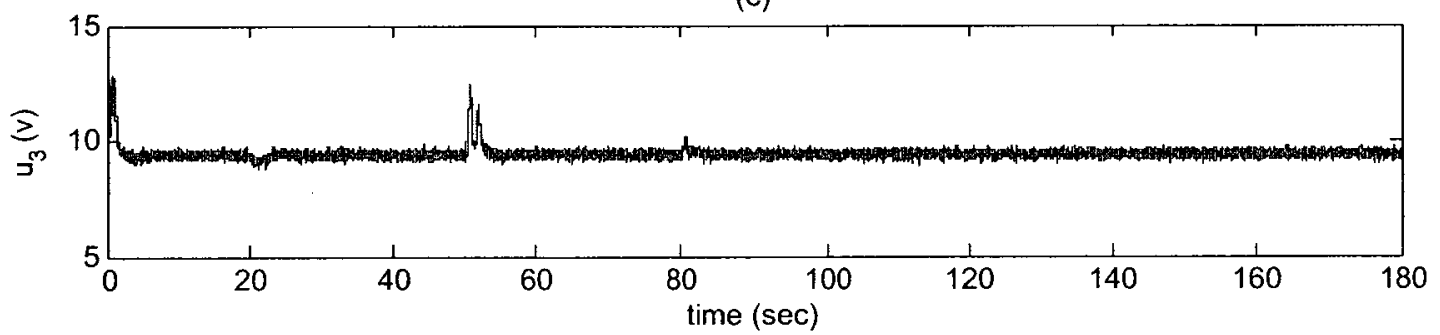

(d)

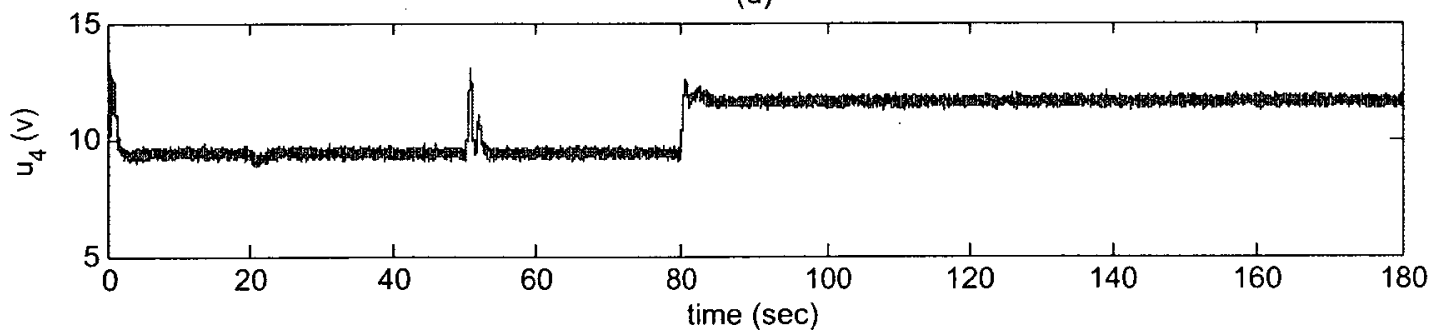

Figure 4.23: Input signals in response to the commanded trajectory corresponding to a $10 \%$ LOE fault in the first and 4 th actuators without the fault recovery mechanism. 
(a)

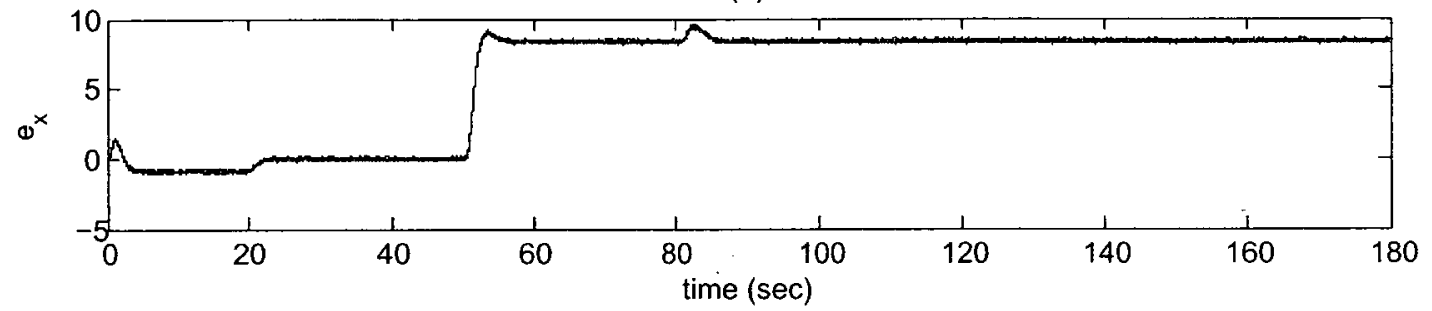

(b)

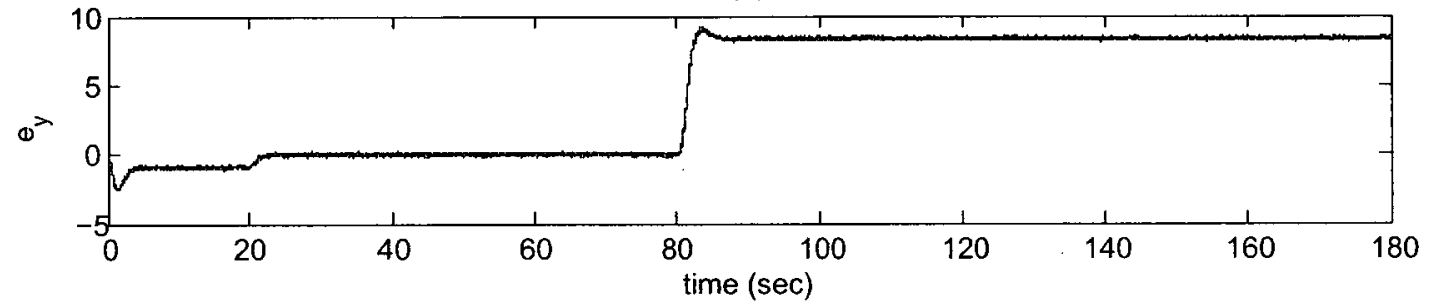

(c)

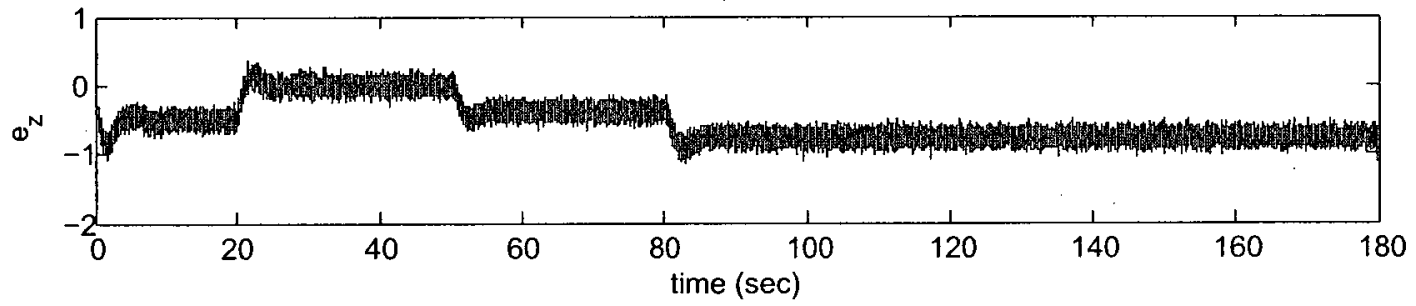

(d)

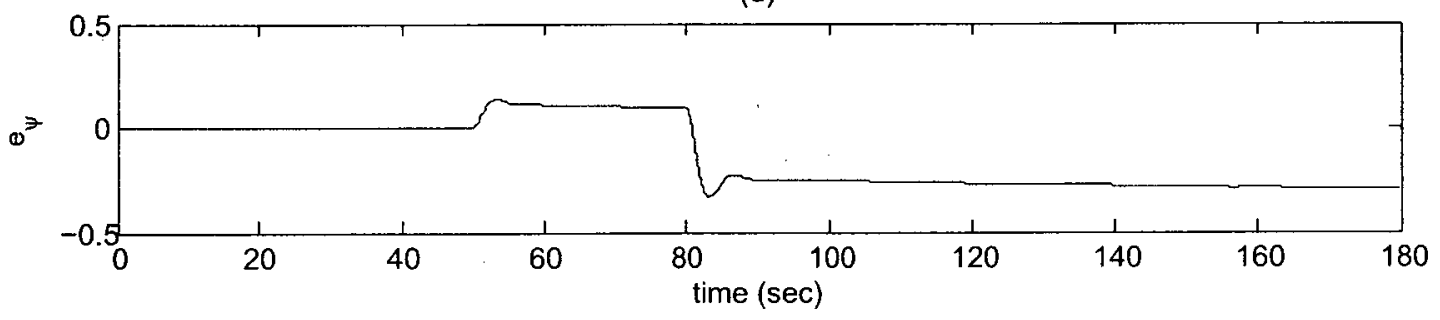

Figure 4.24: Error signals $e_{x}, e_{y}, e_{z}$ measured in meters and $e_{\psi}$ in radians in response to the commanded trajectory corresponding to a $10 \% \mathrm{LOE}$ fault in the first and 4 th actuators without the fault recovery mechanism. 
(a)

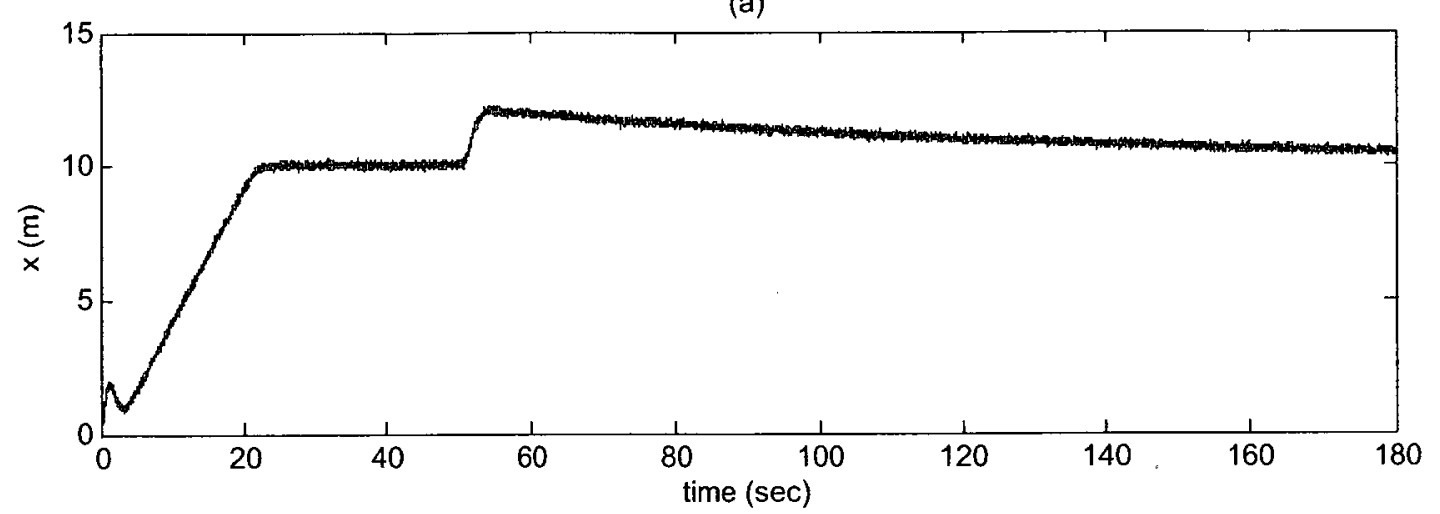

(b)

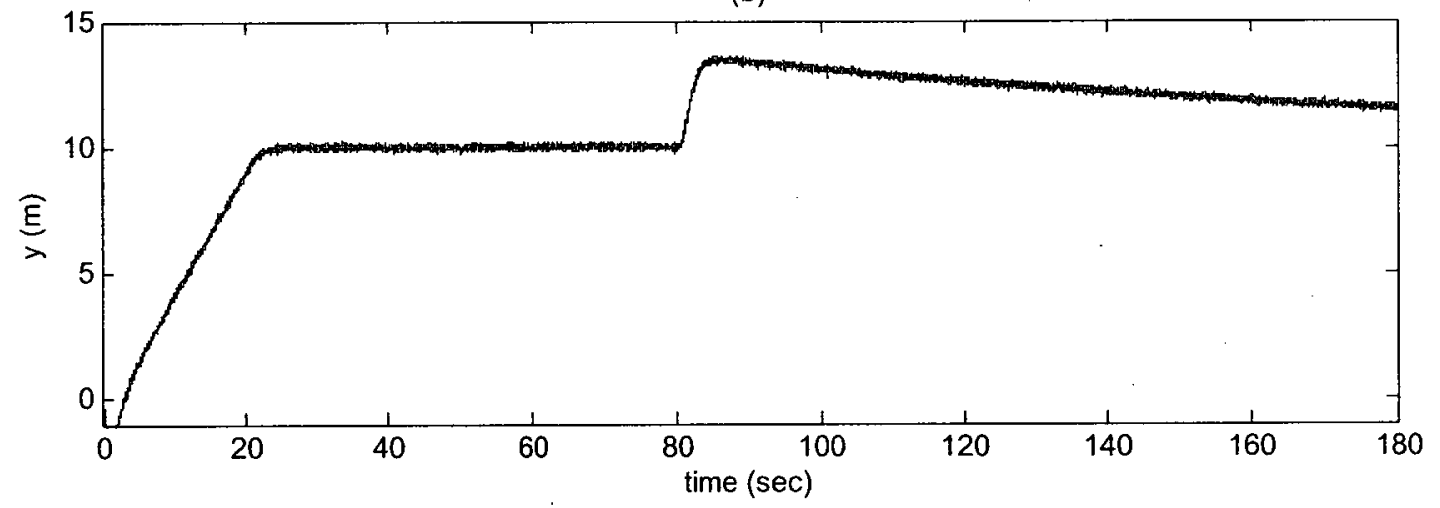

(c)

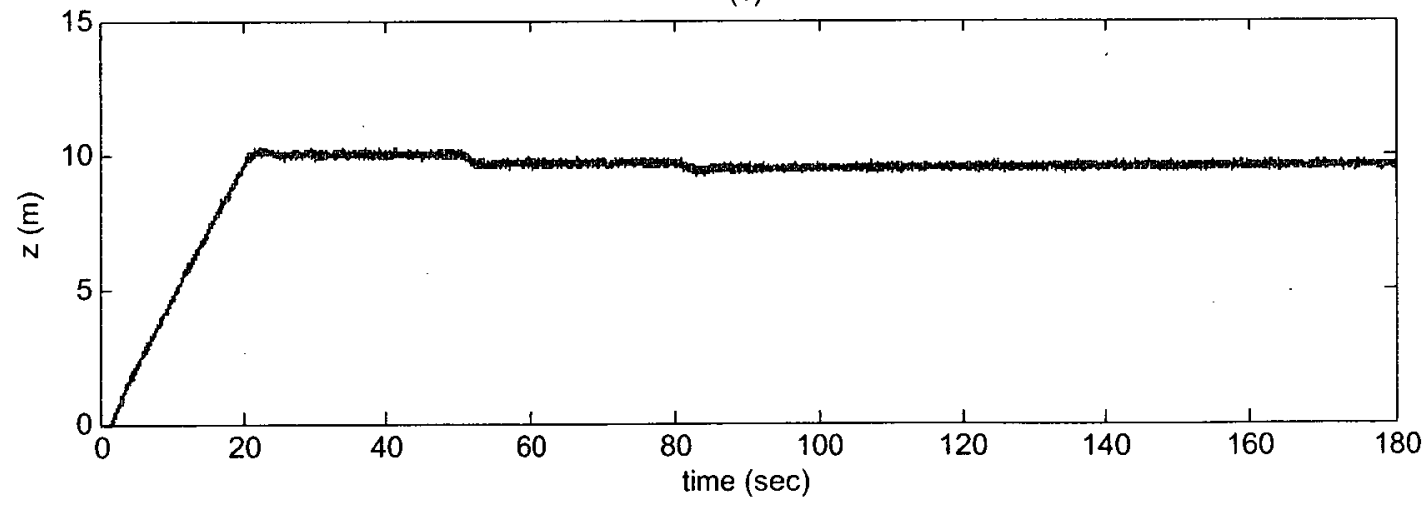

Figure 4.25: Linear position in response to the commanded trajectory corresponding to a $10 \%$ LOE fault in the first and 4 th actuator and with the fault recovery mechanism: (a) $\mathrm{x}$, (b) $\mathrm{y}$ and (c) $\mathrm{z}$, measured in meters. 
(a)

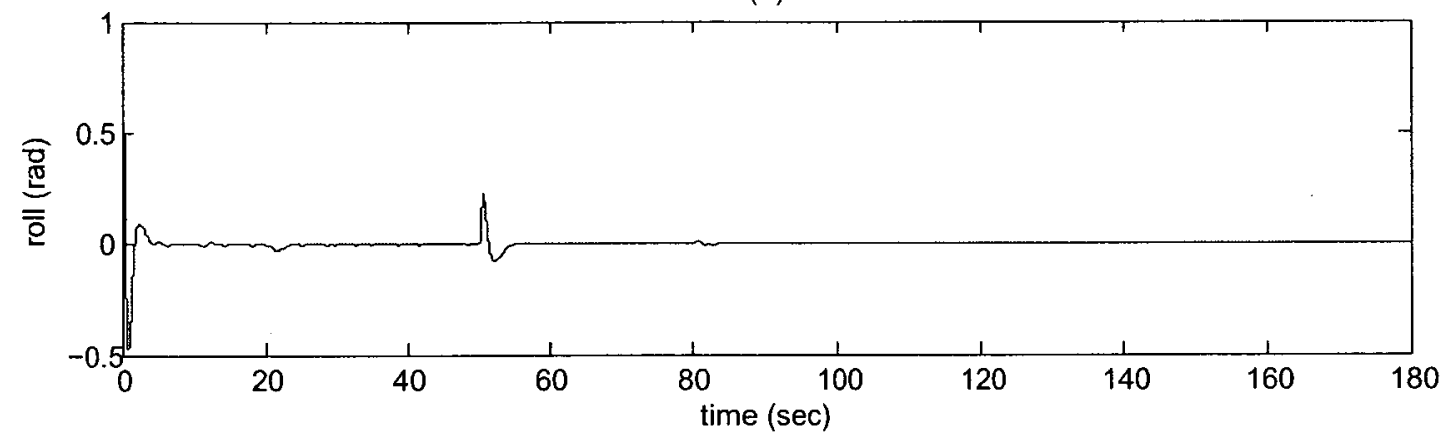

(b)

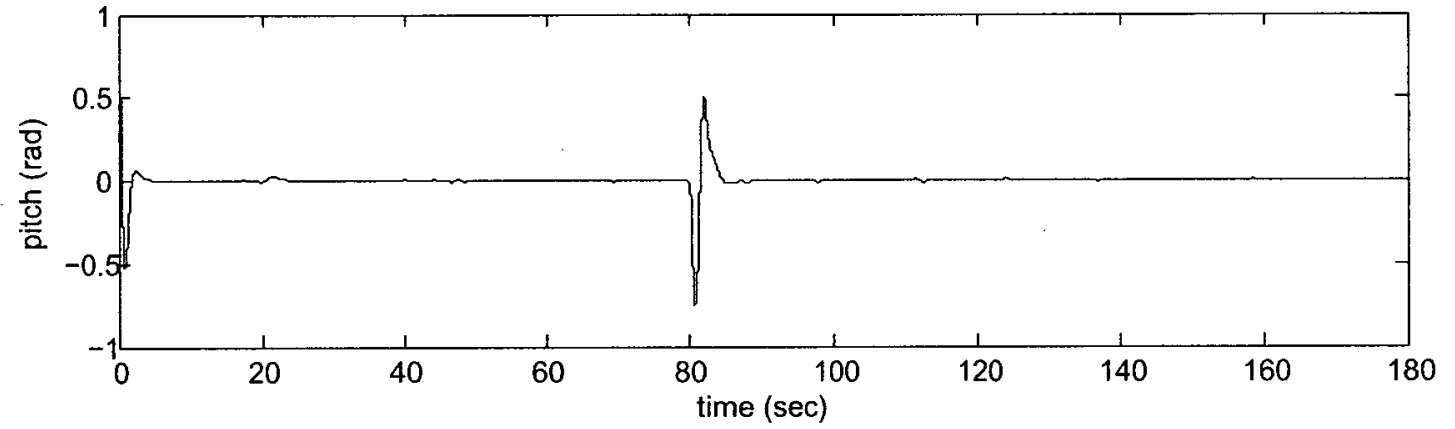

(c)

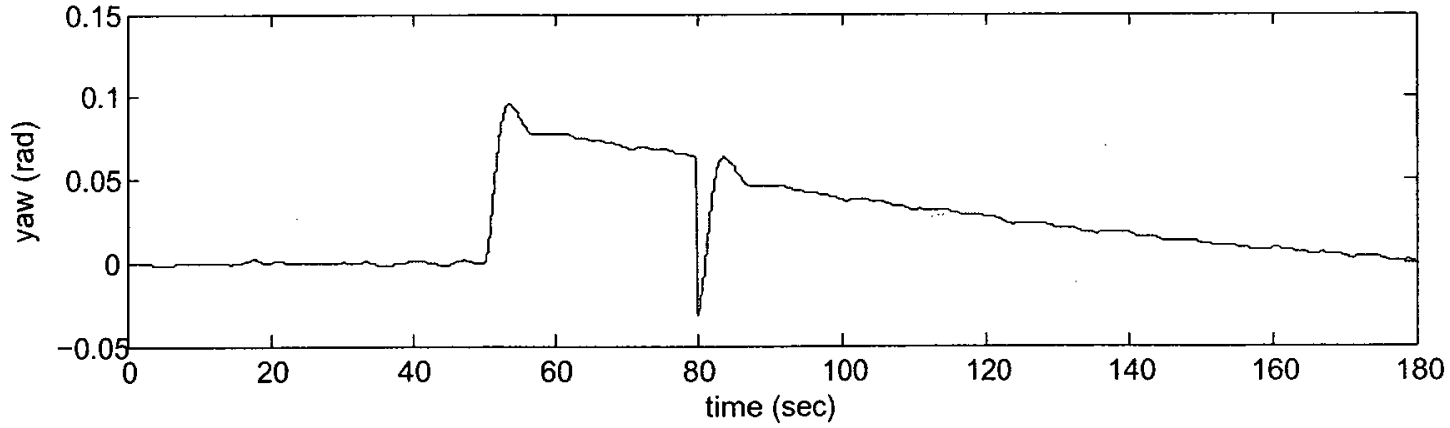

Figure 4.26: Euler angles in response to the commanded trajectory corresponding to a $10 \% \mathrm{LOE}$ fault in the first and 4 th actuators and with the fault recovery mechanism: (a) Roll (rad), (b) Pitch (rad) and (c) Yaw (rad). 
(a)

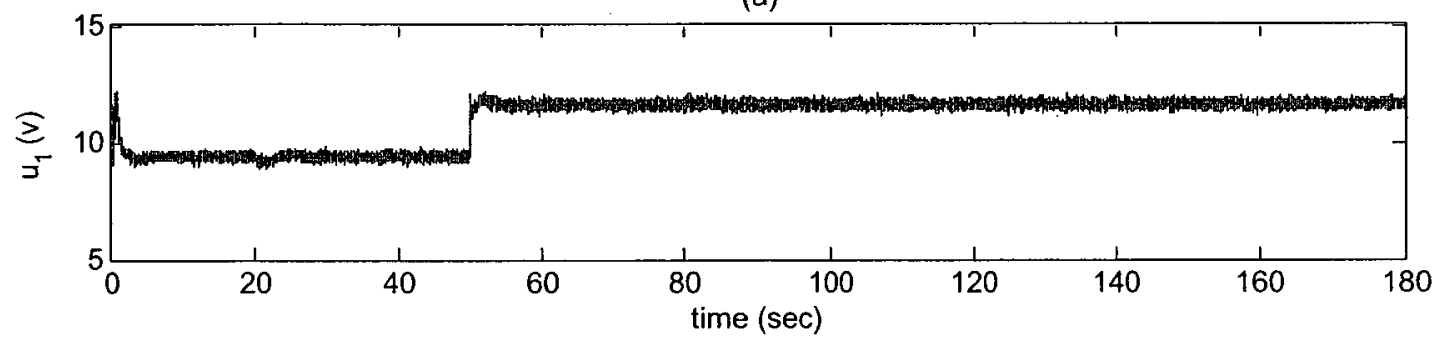

(b)

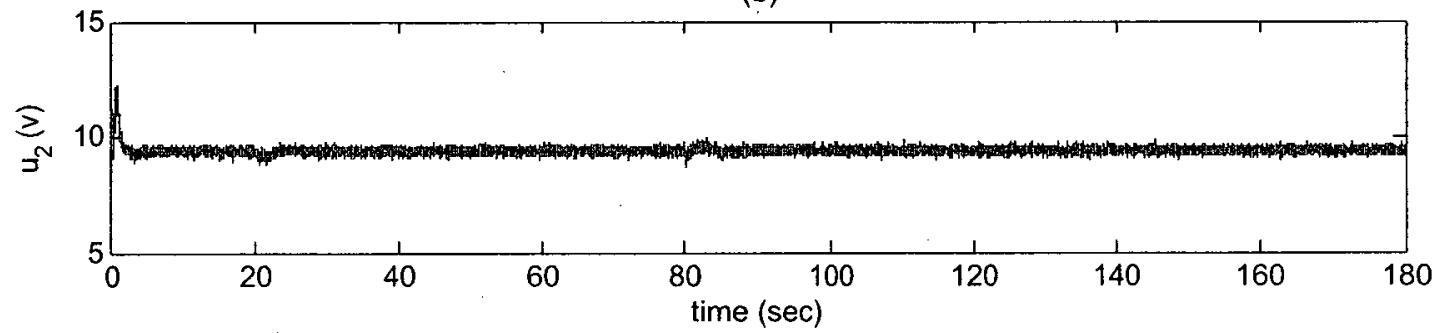

(c)

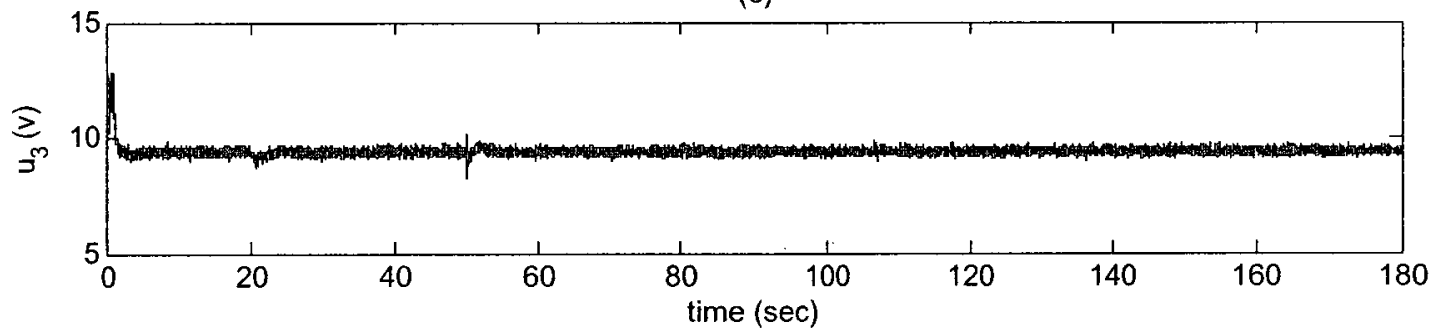

(d)

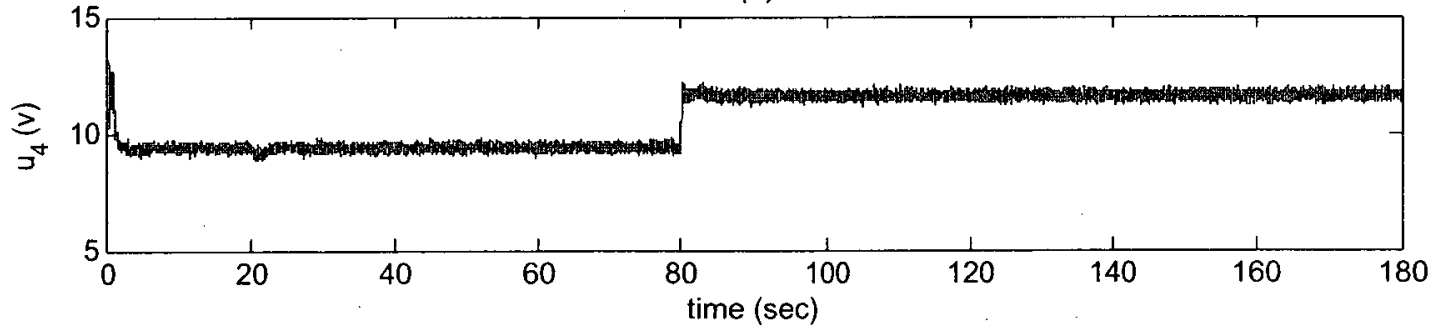

Figure 4.27: Input signals in response to the commanded trajectory corresponding to a $10 \%$ LOE fault in the first and 4th actuators with the fault recovery mechanism. 
(a)

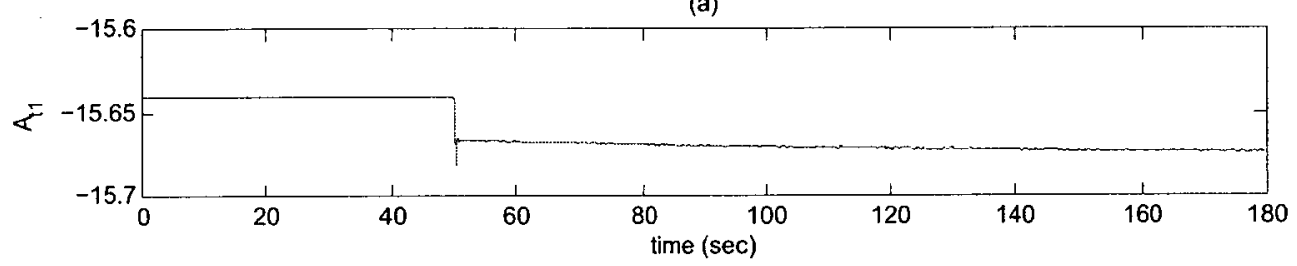

(b)

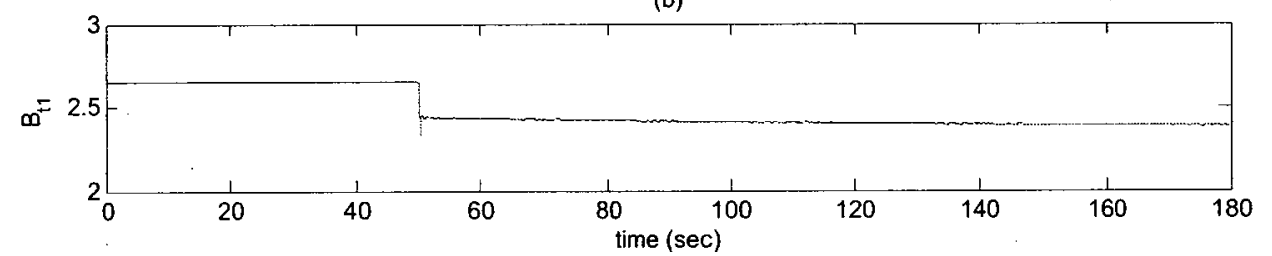

(a)

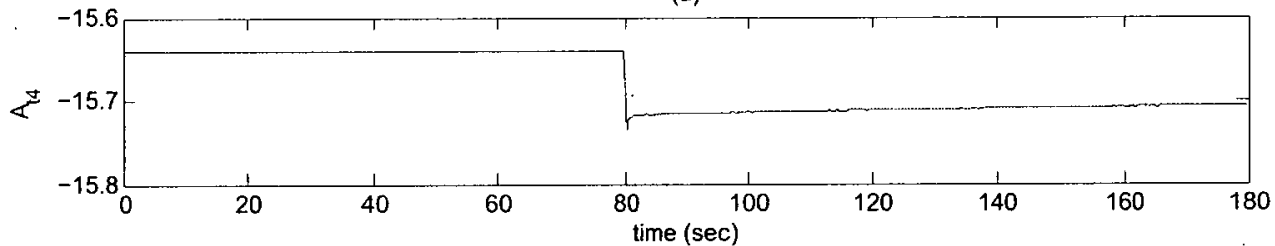

(b)

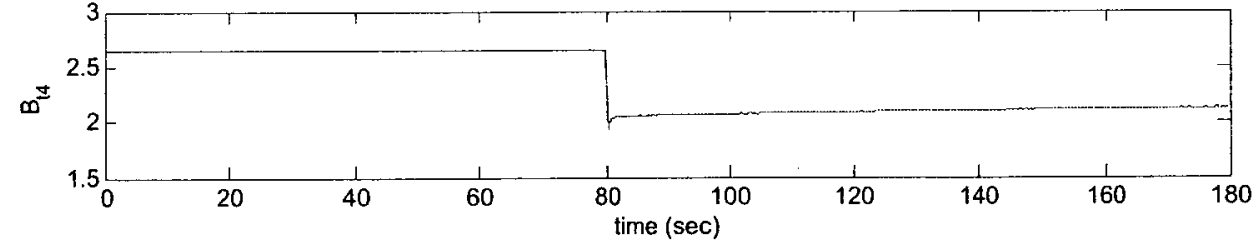

Figure 4.28: Estimated parameters in response to the commanded trajectory corresponding to a $10 \%$ LOE fault in the first and 4 th actuators with the fault recovery mechanism: (a) $A_{t 1}$ and (b) $B_{t 1}$ 
(a)

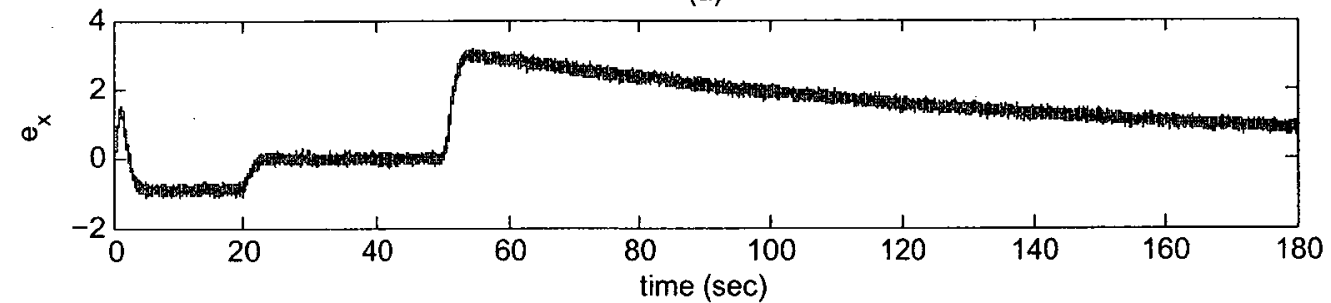

(b)

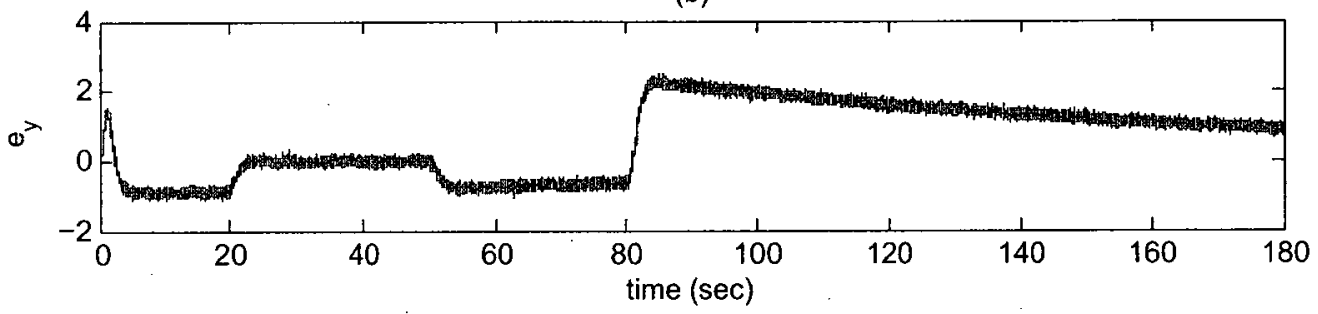

(c)

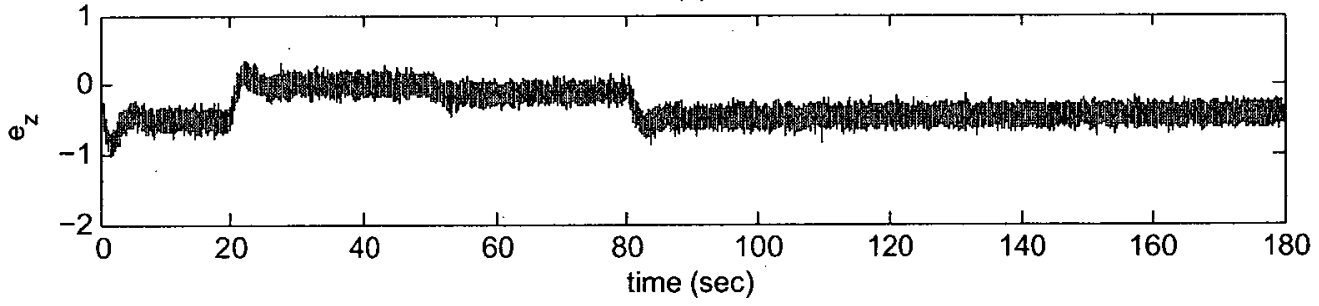

(d)

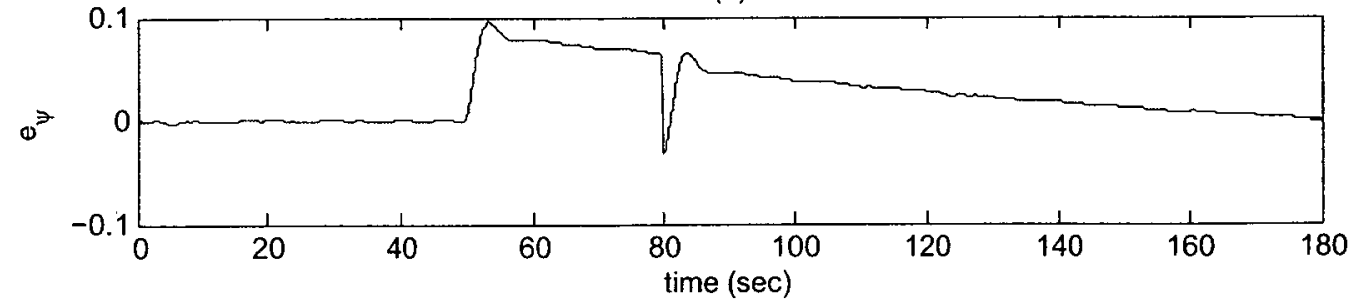

Figure 4.29: Error signals $e_{x}, e_{y}, e_{z}$ measured in meters and $e_{\psi}$ in radians in response to the commanded trajectory corresponding to a $10 \% \mathrm{LOE}$ fault in the first and 4 th actuators with the fault recovery mechanism. 


\section{Chapter 5}

\section{Conclusions and Future Work}

\subsection{Conclusions}

In this thesis, a fault recovery module by employing an adaptive feedback linearization technique is developed and applied to an under-actuated quadrotor aerial vehicle. Partial LOE fault is simulated as variation in certain actuator parameters. A parameter estimation scheme is designed to monitor the performance of the actuators. The stability of the closed-loop system in presence of LOE actuator faults is ensured by proper adaptive update laws.

As the first step, the mathematical model of the quadrotor system is presented by employing the Newton-Euler formulations as well as the dynamic equations of the actuators are formally incorporated. A comprehensive and detailed models on the coordinate systems, the kinematics and the dynamics model of the quadrotor and the actuators are also provided.

The dynamics of the quadrotor model are partitioned into four semi-decoupled subsystems by considering some approximations at the hovering condition. Furthermore, the dynamics governing the input moments to the quadrotor are derived and considered in the controller design for the system. Feedback linearization and LQR 
techniques are employed to design controllers for the nonlinear subsystems. A formal analysis is conducted to show that the closed-loop system is asymptotically stable. Simulation results are performed in MATLAB simulink and presented to elucidate the performance of the nominal controller under different trajectories in healthy conditions. In order to simulate a more realistic environment, we have added noise to the output signals as well as inputs. Furthermore, the robustness of the designed controller is evaluated through simulations and by assuming variations in some of the model parameters. The simulations show satisfactory performance of the designed controller for the healthy quadrotor according to the design criteria.

Different types of faults namely LIP, HOF, float and LOE in actuators are discussed. A complete analysis of the effects of each fault on the quadrotor system is provided. The solution proposed for the fault recovery in actuators is shown to be effective for the recovery from LOE fault. The feedback linearization controller developed for the healthy quadrotor is generalized to compensate partial LOE fault effects on the system. This is accomplished by introducing a parameter estimation algorithm and deriving proper update laws for the parameters subject to changes due to the presence of the LOE fault. The algorithm is extended to monitor the performance of all the four actuators to recover from multiple occurrences of LOE faults. Stability analysis of the closed-loop system with the embedded fault recovery scheme is also provided. A discussion on the modeling of the LIP, HOF and float faults is provided that indicates that our proposed fault recovery method is inadequate for recovering from these types of faults. This is due to the fact that the quadrotor is an under-actuated system and there is no redundant actuator to compensate for the effect of these types of faults.

Simulation results are prescnted to show the effectiveness of our proposed fault recovery methods in presence of the LOE fault in actuators. Different fault scenarios with different severities of the LOE faults in one actuator are discussed 
and the performance of the closed-loop system with and without our proposed fault recovery strategy is evaluated through simulations. In some of the fault scenarios the system becomes unstable without the fault recovery module, however, by including the fault recovery algorithm the system remains stable and the output error signals remain bounded and finally converge to zero within a sufficiently fast time.

The recovery algorithm is also evaluated for the case of multiple LOE fault occurrences in two different actuators. Due to the capability of our proposed generalized fault recovery algorithm to accommodate multiple faults in different actuators, the results obtained through simulation show satisfactory performance of the controller.

A quantitative evaluation of the results is also made in order to compare the performance of the quadrotor system under faults with and without our proposed recovery strategy. The mean and standard deviations of the output error signals are used for this analysis. The results demonstrate that the performance of the system under recovery is considerably more efficient than the system under fault with no recovery solution.

\subsection{Future work}

The present work has made some assumptions and approximations to partition the nonlinear model of the quadrotor into four semi-decoupled subsystems. The dynamic equations for the input moments to the quadrotor are also linearized. It is clear that more accuracy is obtained by considering the fully nonlinear model of the system for designing the controllers.

Environmental disturbances, such as wind gust can also be included in the model of the quadrotor. This could be performed by considering the wind gust as an additive torque in a certain direction. Parameters of the wind gust should be 
estimated by employing proper estimation methods so that the designed controllers are capable of canceling the effects of this disturbance on the system.

Physical limits and and bounds on the input voltages to the actuators may also be incorporated in the designed fault recovery algorithm so that the results are more realistic and closer to the actual quadrotor system.

The analysis of the system should incorporate the effects of delay in fault detection and inaccuracies by the fault detection and isolation module. This would make it possible to determine the maximum allowable delays in the fault recovery or fault detection system to guarantee the stability of the closed-loop system.

Another direction of the further work can also be on developing a fault recovery algorithm in case of LIP, HOF and float faults in actuators for the under-actuated quadrotor system. Control allocation techniques may be a proper method to investigate for the fault recovery from these types of faults.

The concentration of this work was on recovery from faults that may occur in actuators. Faults in sensors of the system may also affect the performance of the system. Hence, another direction of future studies can be on developing fault recovery strategies to recover from faults in different sensors of the quadrotor aerial vehicle. 


\section{Bibliography}

[1] S. Bouabdallah, Design and Control of Quadrotors With Application to Autonomous Flying. PhD thesis, Ecole Polythechnique Federale De Lausanne, 2007 .

[2] A. Tayebi and S. McGilvray, "Attitude stabilization of a four-rotor aerial robot," 2004.

[3] P. Adigbli, "Nonlinear attitude and position control of a micro quadrotor using sliding mode and backstepping techniques," in European Micro Air Vehicle Conference and Flight Competition, 2007.

[4] L. Derafa, T. Madani, and A. Benallegue, "Dynamic modelling and experimental identification of four rotors helicopter parameters," in IEEE International Conference on Industrial Technology, pp. 1834-1839, 2006.

[5] P. Castillo, R. Lozano, and A. Dzul, Modelling and Control of Mini-Flying Machines. Springer, 2005.

[6] V. Venkatasubramanian, R. Rengaswamy, K. Yin, S. N., and K. d, "A review of process fault detection and diagnosis part i: Quantitative model-based methods," in Computers and Chemical Engineering, 2003.

[7] C. N. Jones, "Reconfigurable flight control, first year report." University of Cambridge, 2005. 
[8] R. Patton, "Fault-tolerant control systems: The 1997 situation," in IFAC Symposium on Fault Detection Supervision and Safety for Technical Processes, 1997.

[9] Y. Zhang and J. Jiang, "Bibliographical review on reconfigurable fault tolerant control systems," in In Proceedings of the 5th IFAC Symposium on Fault Detection, Supervision and Safety for Technical Processes, pp. 265-276, 2003.

[10] J. D. Boskovic and R. K. Mehra, "A multiple model-based reconfigurable flight control system design," in Proceedings on the 37th IEEE Conference on Decision and Control., 1998.

[11] M. Gopinathan, J. D. Boskovic, R. K. Mehra, and C. Rago, "A multiple model predictive scheme for fault-tolerant flight control design," in Proceedings of the 37th IEEE Conference on Decision and Control, 1998.

[12] P. S. Maybeck, "Multiple model adaptive algorithms for detecting and compensating sensor and actuator/surface failures in aircraft flight control systems.," International Journal of Robust and Nonlinear Control, 1998.

[13] F. W. Burcham, J. J. Burken, T. A. Maine, and J. Bull, "Emergency flight control using only engine thrust and lateral center-of-gravity offset." Technical report, NASA, 1997.

[14] M. Bodson, "A reconfigurable nonlinear autopilot," in Proceedings of the AIAA Guidance, Navigation, and Control Conference, 2002.

[15] M. Bodson, "Multivariable adaptive algorithms for reconfigurable flight control," IEEE Transactions on Control Systems Technology, 1997.

[16] A. T. Vemuri and M. M. Polycarpou, "Neural network based robust fault diagnosis in robotic systems," IEEE Transactions on Neural Networks, vol. 8, pp. $1410-1420,1997$. 
[17] H. K. Khalil, Nonlinear Systems. Prentice Hall, 2002.

[18] J. J. Burken, P. Lu, Z. Wu, and C. Bahm, "Two reconfigurable flight-control design methods: Robust servomechanism and control allocation," Journal of Guidance, Control and Dynamics, 2001.

[19] D. Enns, "Control allocation approaches," in Proceedings of Guidance, Navigation and Control Conference, 1998.

[20] M. W. Oppenheimer, D. B. Doman, and M. A. Bolender, "Control allocation for over-actuated systems." AIR FORCE RESEARCH LAB WRIGHTPATTERSON AFB OH AIR VEHICLES DIRECTORATE, 2006.

[21] J. B. Davidson, F. J. Lallman, and W. T. Bundick, "Integrated reconfigurable control allocation," in Proceedings of AIAA Guidance, Navigation, and Control Conference, 2001.

[22] Y. Zhang, V. S. Suresh, B. Jiang, and D. Theilliol, "Reconfigurable control allocation against aircraft control effector failures," in 16th IEEE International Conference on Control Applications Part of IEEE Multi-conference on Systems and Control, 2007.

[23] Y. Zhang, C. A. Rabbath, and C.-Y. Su, "Reconfigurable control allocation applied to an aircraft benchmark model," in 2008 American Control Conference, 2008.

[24] W. A. Kwong, K. M. Passino, E. G. Laukonen, and S. Yurkovich, "Expert supervision of fuzzy learning systems for fault tolerant aircraft control," in Proceedings of the IEEE, 1995.

[25] RC Toys, available at http://www.rctoys.com/. 
[26] I. Kroo, F. Prinz, and M. Shantz, "The mesicopter: A miniature rotorcraft concept." 2001.

[27] G. Fay, "Derivation of the aerodynamic forces for the mesicopter simulation." 2001.

[28] G. Hoffmann, D. G. Rajnarayan, S. L. Waslander, D. Dostal, J. S. Jang, and C. J. Tomlin, "The stanford testbed of autonomous rotorcraft for multi agent control (starmac)," in Proceedings of the 23rd Conference in Digital Avionics Systems, 2004.

[29] P. Pounds, R. Mahony, and P. Corke, "Modelling and control of a quad-rotor robot," in Proceedings of the Australasian Conference on Robotics and Automation, 2006.

[30] N. Guenard, T. Hamel, and V. Moreau, "Dynamic modeling and intuitive control strategy for an $\mathrm{x} 4$-flyer," in Proceedings of 5th International Conference on Control and Automation, 2005.

[31] S. Bouabdallah and R. Siegwart, "Full control of a quadrotor," in Proceedings of the 2007 IEEE/RSJ International Conference on Intelligent Robots and Systems, 2007.

[32] J. How, B. Bethke, A. Frank, D. Dale, and J. Vian, "Real-time indoor autonomous vehicle test environment," IEEE Control Systems Magazine, 2008.

[33] M. Valenti, B. Bethke, G. Fiore, and J. P. H. E. Feron, "Indoor multi-vehicle flight testbed for fault detection, isolation, and recovery," in AIAA Guidance, Navigation and Control Conference, 2006.

[34] I. Cowling, J. Whidborne, and A. Cooke, "Optimal trajectory planning and lqr control for a quadrotor uav," in Proceedings of the International Conference Control, 2006. 
[35] A. Noth, S. Bouabdallah, and R. Siegwart, "Pid vs lq control techniques applied to an indoor micro quadrotor," in Proceedings of International Conference on Intelligent Robots and Systems, pp. 2451 - 2456, 2004.

[36] R. Lozano, P. Castillo, and A. Dzul, "Stabilization of a mini rotorcraft with four rotors," IEEE Control Systems Magazine, pp. 45 - 55, 2005.

[37] S. Bouabdallah, P. Murrieri, and R. Siegwart, "Design and control of an indoor micro quadrotor," in Proceedings of The International Conference on Robotics and Automation (ICRA), 2004.

[38] A. Dzul, P. Castillo, and R. Lozano, "Real-time stabilization and tracking of a four-rotor mini rotorcraft," IEEE Transaction on Control System Technology, p. $510516,2004$.

[39] R. L. P. Castillo and A. Dzul, "Stabilization of a mini rotorcraft having four rotors," in Proceedings of 2004 IEEE/RSJ International Conference on Intelligent Robots and Systems, pp. 2693 - 2698, 2004.

[40] A. Palomino, S. Salazar-Cruz, and R. Lozano, "Trajectory tracking for a four rotor mini-aircraft," in Proceedings of the 44th IEEE Conference on Decision and control, and the European Control Conference, p. 2505 2510, 2005.

[41] A. Tayebi and S. McGilvray, "Attitude stabilization of a vtol quadrotor aircraft," IEEE Transaction on Control System Technology, pp. 562 - 571, 2006.

[42] T. Madani and A. Benallegue, "Backstepping control for a quadrotor helicopter," in Proceedings of 2006 IEEE/RSJ International Conference on Intelligent Robots and Systems, p. 3255 3260, 2006.

[43] T. Madani and A. Benallegue, "Backstepping sliding mode control applied to a miniature quadrotor flying robot," in Proceedings of 32nd Annual Conference on IEEE Industrial Electronics, IECON 2006, pp. $700-705,2006$. 
[44] T. Madani and A. Benallegue, "Control of a quadrotor mini-helicopter via full state backstepping technique," in Proceedings of the 45th IEEE Conference on Decision and Control, pp. 1515 - 1520, 2006.

[45] S. Bouabdallah and R. Siegwart, "Backstepping and sliding-mode techniques applied to an indoor micro quadrotor," in Proceedings of the 2005 IEEE International Conference on Robotics and Automation, 2005.

[46] D. Lee, H. J. Kim, and S. Sastry, "Feedback linearization vs. adaptive sliding mode control for a quadrotor helicopter," International Journal of Control, Automation, and Systems, pp. 419-428, 2009.

[47] H. Bouadi, M. Bouchoucha, and M. Tadjine, "Sliding mode control based on backstepping approach for an uav type-quadrotor," International Journal of. Applied Mathematics and Computer Sciences, pp. 12 - 17, 2007.

[48] R. Xu and U. Ozguner, "Sliding mode control of a quadrotor helicopter," in Proceedings of the 45th IEEE Conference on Decision and Control, 2006.

[49] T. Hamel, N. Guenard, and R. Mahony, "A practical visual servo control for a unmanned aerial vehicle," in Proceedings of IEEE International Conference on Robotics and Automation, p. 1342 1348, 2007.

[50] M. Valenti, G. P. Tournier, and J. P. How, "Estimation and control of a quadrotor vehicle using monocular vision and moire patterns," in AIAA Guidance, Navigation, and Control Conference and Exhibit, 2006.

[51] T. Hamel, N. Metni, and F. Derkx, "Visual tracking control of aerial robotic systems with adaptive depth estimation," in Proceedings of the 44 th IEEE Conference on Decision and Control, and the European Control Conference 2005, p. $60786084,2005$. 
[52] J. P. Ostrowski, E. Altug, and C. J. Taylor, "Quadrotor control using dual camera visual feedback," in Proceedings of the 2003 IEEE International Conference on Robotics and Automation, p. 4294 4299, 2003.

[53] M. G. Earl and R. D'Andrea, "Real-time attitude estimation techniques applied to a four rotor helicopter," in Proceedings of 43rd IEEE Conference on Decision and Control, pp. $3956-3961,2004$.

[54] C. Coza and C. J. B. Macnab, "A new robust adaptive-fuzzy control method applied to quadrotor helicopter stabilization," in NAFIPS Annual meeting of the North American Fuzzy Information Society, pp. 454 - 458, 2006.

[55] M. Tarbouchi, J. Dunfied, and G. Labonte, "Neural network based control of a four rotor helicopter," in Proceedings of IEEE Intrnational Conference on Industrial Technology, p. 1543 1548, 2004.

[56] J. S. Jang, S. L. Waslander, G. M. Hoffmann, and C. J. Tomlin, "Multiagent quadrotor testbed control design: Integral sliding mode vs. reinforcement learning," in Proceedings of IEEE/RSJ International Conference on Intelligent Robots and Systems, 2005.

[57] V. Mistler, A. Benallegue, and N. M'Sirdi, "Exact linearization and noninteracting control of a 4 rotorshelicopter via dynamic feedback," in Proceedings of 10th IEEE International Workshop on Robot and Human Interactive Communication, 2001.

[58] E. Altug, J. P. Ostrowski, and R. Mahony, "Control of a quadrotor helocopter using visual feedback," in Proceedings of the IEEE International Conference on Robotics and Automation, pp. $72-77,2002$.

[59] A. Mokhtari and A. Benallegue, "Dynamic feedback controller of euler angles and wind parameters estimation for a quadrotor unmanned aerial vehicle," in 
Proceedings of IEEE International Conference on Robotics and Automation, 2004.

[60] J. L. Boiffier, The Dynamic of Flight, The Equation. John Wiley and Sons, 1998.

[61] T. Bresciani, "Modelling, identification and control of a quadrotor helicopter," Master's thesis, Lund University, 2008.

[62] A. V. Rao, Dynamics of Particles and Rigid Bodies: a Systematic Approach. Cambridge University Press, 2006.

[63] J. P. Hespanha, "Undergraduate lecture notes on lqg/lqr controller design." 2007.

[64] K. Groves and A. Serrani, "Modeling and nonlinear control of a single-link flexible joint manipulator." available at http://www.ece.osu.edu/ passino/lab5prelabnlc.pdf.

[65] J. J. Slotine, Applied Nonlinear Control. Prentice Hall, 1991. 